\section{Eingeladene Vorträge}

\section{Konservative und mikrochirurgisch-rekonstruktive Therapie von Armlymphödemen: Widerspruch oder ergänzende Therapieoptionen?}

*Rüdiger G.H. Baumeister, Andreas Frick

Plastische-, Hand-, Mikrochirurgie, Chirurg. Klinik u. Poliklinik der LMU München - Großhadern, München, Deutschland

Konservative und operative Therapieansätze können sich unversöhnlich gegenüberstehen, sich ergänzen oder nebeneinander bestehen, wobei pathophysiologische Argumente und objektivierbare Resultate den Ausschlag bei der Beurteilung geben sollten.

Sowohl die konservative Therapie wie auch die operativen Maßnahmen müssen dabei differenziert betrachtet werden.

Die mikrochirurgische autogene Lymphgefäßtransplantation nimmt innerhalb der operativen Maßnahmen insofern eine Sonderstellung ein, als sie eine direkte Rekonstruktion unterbrochener Lymphbahnen und damit eine «Restitutio» anstrebt.

Die Ergebnisse von Volumenmessungen, vergleichende Lymphsequenzszintigraphien und indirekte Lymphographien objektivieren dabei auch bei der Langzeitbeobachtung die Erreichbarkeit dieses Strebens nach Verbesserung bis Normalisierung der Lymphtransportkapazität.

Da die konservative Therapie das gleiche Ziel, nämlich eine Verbesserung der Lymphtransportkapazität mit geringeren invasiven Mitteln anstrebt, ist diese als erste Option zu wählen. Stellt sich nach einer mindestens 6-monatigen konsequenten Therapiedauer jedoch keine dauerhafte Entödematisierung ein, so sollte eine rekonstruktive operative Therapie als zweite Option dem Patienten angeboten werden bevor Sekundärveränderungen in den betroffenen Extremitäten überhand nehmen.

\section{Langzeitverläufe bei Stewart-Trewes-Syndrom}

*Gunther Felmerer ${ }^{1}$, Nestor Torio-Padron ${ }^{1}$, Björn Stark ${ }^{1}$, Etelka Földi ${ }^{2}$ ${ }^{1}$ Abt. Plastische und Handchirurgie, Chirurgische Universitätsklinik, (Direktor: Prof. Dr. med. G. B. Stark), Freiburg, Deutschland

${ }^{2}$ Földi-Klinik, Fachklinik für Lymphologien, Hinterzarten,

\section{Deutschland}

Zielsetzung: Das Angiosarkom ist eine seltene Komplikation bei Patienten mit chronischem Lymphödem. Zuerst beschrieben wurde dieses Syndrom von Stewart und Trewes. Durch eine Auswertung der Krankheitsverläufe könnten sich Strategien zur Prophylaxe und Therapie ableiten lassen. Materialien und Methoden: Aus einem Patientenkollektiv $(\mathrm{n} \geq 15000)$ einer lymphologischen Fachklinik wurden Stewart-TrewesFälle $(\mathrm{n}=10)$ der letzten 28 Jahre retrospektiv durch Analyse der Krankenunterlagen ausgewertet. Ergebnisse: Fünf Patientinnen waren an einem Mammakarzinom erkrankt, zwei Patienten an anderen Tumoren. Zwei Patienten waren an einem primären und eine am sekundären Lymphödem nach Lymphadenektomie erkrankt. Von den Tumorpatienten wurden alle bestrahlt. Im Durchschnitt trat das Sarkom 14,3 Jahre nach dem Auftreten des Lymphödems auf, wobei bei den sekundären Lymphödemen (alle mit Radiatio) bereits nach durchschnittlich 7,5 Jahren der Tumor diagnostiziert wurde (Lymphödempatienten ohne Tumor und ohne Radiatio nach durchschnittlich 33,3 Jahren). Alle Patienten bis auf eine erhielten keine komplexe physikalische Entstauungstherapie, in einem Fall wurde die Lymphdrainage unvollständig durchgeführt. In sieben Fällen wurde die Extremität amputiert, in zwei Fällen fand eine Wide-exzision mit Spalthautdeckung statt, alle Resektionen R0, eine Patientin wurde wegen Metastasierung nicht operiert. Acht von zehn Patienten verstarben im Durchschnitt nach 25 Monaten, eine Patientin ist seit 16 Jahren rezidivfrei, eine wurde vor einem halben Jahr operiert und ist zur Zeit rezidivfrei. Zusammenfassung: Eine adäquate Behandlung des chronischen Lymphödems kann ein Stewart-Trewes-Syndrom verhindern. Hämatom-ähnliche Flecken oder Verhärtungen des Subkutangewebes sind ab dem 5. Jahr nach Ausbruch des Ödemsdann hochverdächtig auf ein Lymphangiosarkom. Die Art der Operation, sofern sie R0 durchgeführt wird, scheint keine Auswirkung auf die Langzeitprognose zu haben.

\section{Sonographisch gesteuerte Hochgeschwindigkeits- biopsie von Brusttumoren: Analyse von 962 Fällen zur Festlegung der notwendigen Stanzbiopsieanzahl zur verlässlichen Tumorcharakterisierung}

Georg Sauer ${ }^{1 *}$, Helmut Deissler ${ }^{1}$, Katrin Strunz ${ }^{1}$, Gisela Helms ${ }^{1}$, Eugenia Remmel ${ }^{1}$, Karin Koretz ${ }^{2}$, Rainer Terinde ${ }^{1}$, Rolf Kreienberg ${ }^{1}$ ${ }^{1}$ Universitätsfrauenklinik, UIm, Deutschland 2Institut für Pathologie der Universität, UIm, Deutschland

Zielsetzung: Ziel dieser Untersuchung war, die Anzahl der unter 3DUltraschallkontrolle durchgeführten Stanzbiopsien zu bestimmen, welche notwendig ist, um ein verlässliches histologisches Ergebnis einer sonographisch nachweisbaren Läsion zu erhalten. Material und Methoden: Über eine Periode von 18 Monaten wurden insgesamt 2044 sonographisch gesteuerte Mammabiopsien an 962 Brustveränderungen durchgeführt. Dazu verwendeten wir eine Hochgeschwindigkeitsbiopsienadel mit einem 15 bzw. 22mm Vorschub. In 660 Fällen schloss sich der Probenentnahme eine offene Exzisionsbiopsie an. Die anderen 302 Fälle wurden über einen Zeitraum von bis zu 36 Monaten klinisch nachuntersucht. Ergebnisse: In 96,2\% der Fälle reichten allein nur drei Stanzzylinder aus, um eine eindeutige histologische Klassifizierung einer Brustveränderung vornehmen zu können. Insgesamt konnte mit einer Sensitivität von $97,9 \%$ und einer falsch negativen Rate von 2,1\% eine identische diagnostische Sicherheit wie bei der offenen Biopsie erreicht werden. Zusammenfassung: Unsere Ergebnisse zeigen, dass drei unter 3D-Ultraschallkontrolle durchgeführte Stanzbiopsien einer Brustläsion ausreichend sind, eine histologisch eindeutige Diagnose zu stellen.

\section{Adjuvante und palliative Therapie beim Mann mit Mammakarzinom}

\section{Ulrich R. Kleeberg}

HOPA, Hämatologisch-onkologische Praxis Altona, Hamburg, Deutschland

2\%o aller Malignome des Mannes oder 6-8\%o aller Mammakarzinome (MK) sind MK des Mannes (MKM). Daher fehlen EBM - gesicherte Therapien. Unabhängig vom Alter sind über $80 \%$ ER positiv. Stadienbezogen ist die Prognose des MKM dem der Frau vergleichbar.

3 adjuvante Phase II-Studien zeigen im historischen Vergleich einen Nutzen von Tamoxifen u/o CMF / FAC. Analog zum MK der Frau wird gegebenenfalls eine 5-jährige Tamoxifentherapie empfohlen, bei ungünstigen Primärstadien ( $\mathrm{pT} 3-4, \mathrm{G} 3, \mathrm{pN}>3, \mathrm{~V} / \mathrm{L}+, \mathrm{H}+++$ ) voraus eine Chemotherapie $(6 \times \mathrm{FAC})$, dann konsolidierende Radiatio.

Palliative Maßnahmen beginnen beim ER positiven MKM mit den bei Frauen empfohlenen endokrinen Therapiesequenzen, wobei bis ins hohe Alter die Testosteron-deprivation (Orchiektomie, GnRH-Analoga) an 1. Stelle steht, nach Elimination der testikulären Testosteronsynthese gefolgt von Aromatasehemmern, HD-Progesteron, Fulvestrant sowie Antiandrogenen (Cyproteronazetat, Flutamid, Bicalutamid, Corticosteroiden, Ketokonazol). Kasuistiken zeigen, dass Medikamente, die sowohl mit dem Östrogen- als auch Testosteron-stoffwechsel interferieren, sequentiell eingesetzt, langfristige Remissionen induzieren können.

\section{KARGER}

Fax +497614520714

(ㄷ) 2004 S. Karger GmbH, Freiburg

@Karger.de www.karger.com/onk

www.karger.com 
Vital bedrohliche Metastasierungen fordern eine (ggf auch initiale) Zytostatikatherapie, wobei MK-sensible Substanzgruppen als Mono- und Polychemotherapien eingesetzt werden, bei HER 2 neu positiven Tumoren mit Trastuzumab. Es besteht der Eindruck, dass, auch durch Alter und Komorbidität bedingt, die individuelle Toleranzbreite gegenüber systemischen Massnahmen geringer ist, als die von Frauen mit identischen Verläufen.

Verantwortlich für die Betreuung der Patienten mit MKM ist der qualifizierte medizinische Onkologe gleich welcher Disziplin. Das widersinnige Beharren von BMG und GKV auf indikationsbezogenem, damit das MKM ausschließendem Einsatz von Onkologica, spiegelt die Kompetenz Deutscher Gesundheitspolitik.

\section{Brustkrebs und Sexualität}

Annette Hasenburg

Universitätsfrauenklinik Freiburg

Zum Zeitpunkt der Diagnosestellung konzentriert sich die Patientin zunächst auf die Therapie und sexuelle Wünsche treten in den Hintergrund. Nach Rückkehr in den Alltag kommen sexuelle Bedürfnisse und deren Beeinträchtigungen wieder in den Vordergrund. Ein verändertes Körperbild oder Hormonentzugserscheinungen können die sexuelle Aktivität wesentlich einschränken und zu Einbussen an Lebensqualität und Unzufriedenheit in der Beziehung führen. Der Arzt, der mit seiner Patientin über sexuelle Probleme spricht, zeigt ihr, dass er für sie eine lebenswerte Zeit erwartet und dass er sich nicht nur für ihr Überleben, sondern auch für die Qualität ihres Lebens interessiert. Dabei muss sich der Arzt bewusst sein, dass die Sichtweise der eigenen Sexualität die Gesprächsführung beeinflusst.

Nach einer Ablatio mammae kann durch Protheseneinlage oder Wiederaufbau mit körpereigenem Gewebe, bei Hormonentzugserscheinungen durch die Verwendung von Vaginalgel oder durch lokale Applikation von Östrogenen Hilfe angeboten werden. Die Wiederherstellung der äußeren Silhouette behebt jedoch nicht die innere Verletzung. Die Störung des innerpsychischen Gleichgewichtes der Patientin und ihrer Partnerschaft müssen berücksichtigt werden. Die bewusste Konfrontation mit der körperlichen Veränderung schafft eine beeinflussbare Wirklichkeit. Trauerarbeit mit Anerkennung des körperlichen Verlustes und der damit verbundenen Gefühle ist ein wichtiger Bestandteil der Krankheitsbewältigung. Ausführliche Informationen und offene Gesprächsangebote, am besten schon vor der Operation, müssen in das Therapiekonzept integriert werden. Die Patientin sollte zur Kommunikation, auch mit ihrem Partner und ihren Kindern ermutigt werden.

Zusammenfassung: Eine adäquate Wiederherstellung körperlicher Intimität kann die Lebenszufriedenheit erheblich verbessern und die Ausbildung sekundärer Ängste verhindern. Zu einer patientengerechten onkologischen Versorgung gehören deshalb Gesprächsangebote über sexuelle Probleme.

\section{Prävention des Mammakarzinoms durch Lebensführung}

\section{Ulrich R. Kleeberg}

HOPA, Hämatologisch-Onkologische Praxis Altona, Hamburg, Deutschland

Epidemiologische und prospektive Erhebungen belegen, dass die überwiegende Mehrzahl maligner Erkrankungen durch Lebensweise und Umweltfaktoren verursacht wird, individuell modifiziert durch eine unterschiedlich effiziente genetische und immunologische Abwehr. Beim Mammakarzinom (MK) stehen Fehlernährung, Bewegungsmangel und endokrine Faktoren obenan.

Schätzungen des World Cancer Research Funds und Deutschen Instituts für Ernährungsforschung zufolge (1999) ließe sich die MK-Inzidenz durch eine nachhaltige Korrektur der Lebensgewohnheiten um 2/3, in Deutschland von jährlich zirka 46 auf 15 Tausend senken. Prospektive Studien zeigen sogar bei manifester Erkrankung günstigere Verläufe mit ansteigender Überlebenszeit.

Seit Jahren bemühen sich unsere wissenschaftlichen Gesellschaften (DKG, DGHO, DGS, DGE), Ärztliche Körperschaften (ZI der KBV) und Verbraucherschutz ihren Einfluß geltend zu machen, vergebens: Wie in anderen Industrieländern nimmt auch in Deutschland Überernährung und pro Kopf Konsum an Fleisch und Fett weiter zu, der von Obst und Gemüse ab. Länder Asiens, Ost- und Südeuropas mit historisch niedriger MK Inzidenz gleichen sich in Lebensweise und damit deren fatalen Folgen unseren Verhältnissen an.

Erstmals mit dem GMG wurde Prävention gesetzlich verankert, eine angesichts der demographischen Entwicklung längst überfällige Initiative. Aber auch sie wird ohne umfassende präventive Neuordnung des Gesundheitswesens ein Lippenbekenntnis bleiben. Diese muß, lebensbegleitend konzipiert, frühzeitig in Kindergarten und Schule von Ökotrophologen individuell supervidiert und mit DMP -MK und -Diabetes verbunden werden. Jugendschutzuntersuchungen, Versicherungs-boni, insbesondere aber die hausärztliche Kompetenz und Verantwortung sind weiterzuentwickeln. Ärzteschaft und Lehrkörper kommen, unterstützt von Gesundheitspolitik, Kultusbehörden, «Gesundheitskassen», Pharmazie und Presse diese Aufgabe zu. Aber ist unsere Gesellschaft inzwischen reif für präventives Denken?

\section{Kombination von Chemo- und Strahlentherapie}

*Christine Richter, Jürgen Dunst

Klinik für Strahlentherapie Martin-Luther-Universität, Halle/Saale, Deutschland

Hintergrund: In multidisziplinären Protokollen werden Bestrahlung und Chemotherapie zunehmend in Kombination eingesetzt. Für die lokale und systemische Wirkung spielen die Wahl der Medikamente und die Sequenz eine Rolle. Methodik und Ergebnisse: Chemo- und Strahlentherapie werden kombiniert, um sowohl die systemische als auch lokale Kontrolle zu verbessern (beide Modalitäten wirken unabhängig voneinander sog. «räumliche Kooperation»). Für beide Verfahren gilt in der adjuvanten Therapie, dass sie umso erfolgreicher sind, je früher sie postoperativ eingesetzt werden. Ferner spielt die Intensität der Behandlung (bei der Strahlentherapie die Gesamtbehandlungszeit) eine Rolle. Wegen zum Teil überlappender Toxizitäten zwischen Bestrahlung und Chemotherapie (Kardiotoxizität durch Anthrazykline und Taxane, Haut- und Lungentoxizität) sind sequentielle Regime (also z.B. $4 \times$ AC, danach RT) oder Sandwich-Regime (z. B. $3 \times$ CMF, dann RT, dann $3 \times$ CMF) im Regelfall am günstigsten. Andererseits kann bei simultaner Applikation von Bestrahlung und Chemotherapie ein zusätzlicher strahlensensibilisierender Effekt genutzt werden (d. h. die lokale Wirkung der simultanen Kombination ist besser als die einfache Addition der Einzeleffekte beider Modalitäten, sog. «Radiosensibilisierung»). Es ist sinnvoll, diese lokale Wirkungsverstärkung auszunutzen, wenn ein besonders hohes lokales Rückfallrisiko oder ein inoperabler Tumor bestehen und das lokale Risiko im Vordergrund steht. Bei simultaner Radiochemotherapie müssen die verwendeten Zytostatika dem Fraktionierungsregime der Bestrahlung in der Dosierung und Applikationsweise angepasst und sollten nach ihrer radiosensibilisierenden Wirkung ausgewählt werden. Diese Regime eignen sich auch für die palliative Therapie von symptomatischen Metastasen. Schlussfolgerungen: Bei einer Kombination von Strahlen- und Chemotherapie muss das Behandlungsziel klar definiert und die Wahl der Medikamente und Sequenz diesem angepasst werden.

\section{Arzneimittelinteraktionen in der onkologischen Praxis}

\section{Ulrich R. Kleeberg}

Hämatologisch-Onkologische Praxis Altona, Hamburg, Deutschland

Der Einsatz wirkungsvoller Arzneimittel (AM) in der Onkologie ist durch eine hohe Rate unerwünschter Effekte belastet, sei es durch Fehldosierungen, Interaktionen und /oder individuelle Stoffwechselbesonderheiten. Studien legen nahe, dass bei jährlich rd. 6 Mio. stationär behandelten Patienten zirka 57 Tsd. Todesfälle infolge unerwünschter Arzneimittelwirkungen (UAW) eintreten. Da die Mehrzahl Tumorkranker älter als 65 Jahre ist, sind geriatrische Aspekte von kritischer Bedeutung.

Die Pharmakogenetik variiert von Patient zu Patient, auch innerhalb einheitlicher Populationen. Sie bestimmt die Aktivität der Transportmechanismen (z.B. P-Glykoprotein), der Pharmakokinetik und -dynamik.

AM konkurrieren als Substrate um zelluläre Aufnahme- wie Eliminationsprozesse sowie bei ihrem enzymatischen Abbau. Dabei spielt die 
Konkurrenz um Cytochrom P Oxydasen im Sinne einer Wirkungsverstärkung als auch -minderung eine entscheidende Rolle.

Klinisch relevant sind Interaktionen von Antiemetika, spez. Setronen mi Zytostatika, Kardiaka, Psychopharmaka usw. Sie können z.B. die AUC von Cisplatin oder Cyclophosphamid signifikant mindern. Das kardiotoxische Potential von Setronen spielt insbesondere bei der Interaktion mit QT-Zeit verlängernden Kardiaka eine Rolle, wobei Rhythmusstörungen insbesondere ältere Patienten mit weiteren endogenen oder exogenen Risikofaktoren gefährden.
In der Schmerztherapie sind pharmakokinetische Interaktionen von Opioiden mit Psychopharmaka, Antiphlogistika und Antibiotika relevant. Kombinationen von Opioiden können zu konkurrierenden Blockaden von Opioid-partial- und / oder Vollagonisten und damit zum Wirkungsverlust führen.

Die klinische Pharmakologie erscheint als Spezialfach der medizinischen Onkologie in der Weiter- und Fortbildung nur unzureichend. Eine optimale Versorgung Krebskranker läßt sich aber durch eine interprofessionelle Kooperation mit klinischen Pharmazeuten insbesondere im Rahmen interdisziplinärer onkologischer Arbeitskreise sicherstellen. 


\section{Freie Vorträge: Operative Therapien I/II}

V1

Individualisierte Operationstechniken zur Optimierung der brusterhaltenden Therapie des Mamma-Carcinoms

${ }^{*}$ A. Abdallah, S. Teubner, T. Hassler, Y. Saklaoui

Universitätsfrauenklinik der Ruhr-Universität Bochum, Marienhospital Herne, Herne, Deutschland

Die brusterhaltende Therapie erfordert ein individualisiertes Vorgehen. Der operative Eingriff ist in erster Linie vom klinischen und histo-pathologischen Befund sowie von Brustgröße und Tumorsitz abhängig. Die Indikation zur BET ist nur sinnvoll, wenn die Brust nach dem Eingriff onkologisch sicher ist und ohne größere ästhetische Defekte erhalten werden kann. Zielsetzung: Unterstützung der ln-sano-Resektion mit sofortigem Volumenersatz und Wiederherstellung einer natürlichen Brustgröße und -form, Vermeidung der R1-Resektionen sowie von kosmetischen Defekten. Methodik: Zusammenführung von formverändernden oder formwiederherstellenden Eingriffen bei onkologischen Operationen der Brust. Der Volumenersatz erfolgt durch lokales oder transplantiertes Gewebe: a) lokales Gewebe wird verwendet bei Verfahren der tumorlageradaptierten Mastopexie oder Reduktionsplastik, b) transplantiertes Gewebe bei der BET durch einen Latissimus dorsi-Lappen. Kontraindikation zur brusterhaltenden Therapie: Inkomplette Tumorresektion, multizentrisches Carcinom oder DCIS, ausgedehnte Lymphangiosis carcinomatosa oder Blutgefäßeinbruch, ungünstige Brust-Tumor-Relation, inflammatorisches Mamma-Carcinom, Lokalrezidive nach BET. Ergebnisse: Demonstration anhand von Dias. Schlussfolgerung: Bei richtiger lndikationsstellung ist die brusterhaltende Therapie sicher und eine Alternative zur der Mastektomie. Der Einsatz plastisch-chirurgischer Techniken ermöglich die Rekonstruktion auch größerer Brustdrüsendefekte. Die Patientinnen mit relativer Kontraindikation für BET, wie eine ungünstige Relation zwischen Brust- und Tumorgröße oder zentralem Tumorsitz, können durch individualisierte Techniken brusterhaltend therapiert werden. Die genaue präoperative Planung der Operationstechnik und Schnittfigur unter Berücksichtigung der Brustform und -größe, sowie des Tumorsitzes und -größe vermeidet Mißerfolge bei der BET und führt zu einer hohen lokalen onkologischen Sicherheit.

V2

Der freie TRAM (Tranverse Rectus Abdominis Myocutaneous) Lappen: Standard für die autologe Brustrekonstruktion

${ }^{*}$ C. Andree ${ }^{1}$, M. Voigt ${ }^{2}$, A. Wenger ${ }^{1}$, G. B. Stark ${ }^{1}$

${ }^{1}$ Abteilung Plastische und Handchirurgie,

Chirurgische Universitätsklinik Freiburg, Freiburg, Deutschland

${ }^{2}$ Praxis für Plastische Chirurgie, Freiburg, Deutschland

Einleitung: Es werden in der vorliegenden retrospektiven Studie unsere Erfahrungen mit dem mikrochirurgisch freiem TRAM in der primären und sekundären Brustrekonstruktion dargestellt. Material und Methoden: Eine Rekonstruktion mit einem freien TRAM Lappen erfolgte bei Patientinnen, die eine autologe Brustrekonstruktion wünschten. Die Vorraussetzungen, wie ausreichendes abdominelles Gewebe und eine Durchgängikeit der Arteriae epigastriae profundae inferiores (Doppler-Sonographie), mußten gegeben sein. Als Anschlußgefäße wurden die Thoracica-interna-Gefäße bevorzugt. Die Operationen wurden synchron in zwei Teams durchgeführt. Ergebnisse: In den Jahren 1995 bis 2003 wurden in unserer Abteilung 81 Patienten mit einem freiem TRAM Lappen rekonstruiert Das Durchschnittsalter betrug 50,2 Jahre, wobei die jüngste 26 und die älteste 71 Jahre alt war. 66 Patientinnen erhielten eine unilaterale, 6 Patientinnen eine bilaterale Rekonstruktion. Primärrekonstruktionen wurden bei 32 Patienten durchgeführt, bei 49 Patienten erfolgte eine Sekundärrekonstruktion. Als Anschlußgefäße wurden 46 mal die vasa thoracica internae und 35 mal die vasa thoracodorsalis verwendet. Als Komplikationsraten $(\mathrm{n}=81)$ traten 5,6\% Lappenverluste, 8,3\% mikrochirurgische Revisionen, 11,3\% Hämatome, Wundheilungsstörungen bei 7,0\% und Hernien wurden in 5,6\% der Fälle auf. Zusammenfassung: Der freie TRAM ist ein sicherer Lappen zur Brustrekonstruktion und als Standardlappenplastik in Zentren geeignet. Verglichen mit gestielten Lappenplas- tiken hat die mikrochirugische Form eine bessere Duchblutung und dadurch mehr Gewebevolumen welches für die Rekonstruktion verwendet werden kann. Ferner besteht die Möglichkeit den Lappen uneingeschränkter zu platzieren um die Brustformung durchführen zu können und findet eine geringere Hebemorbidität an der Bauchwand.

\section{V3 \\ Die autologe Gewebetransplantation (TRAM / DIEP) als Therapieoption bei Kapselfibrose der Brust}

\section{*Simone Brüner ${ }^{1}$, Onno Frerichs ${ }^{1}$, Wolfgang Schneider ${ }^{2}$, Hisham Fansa ${ }^{1}$}

${ }^{1}$ Klinik für Plastische, Rekonstruktive and Ästhetische Chirurgie (Chefarzt PD Dr. med. H. Fansa), Städtisches Klinikum Bielefeld Mitte, Bielefeld, Deutschland

${ }^{2}$ Klinik für Plastische, Wiederherstellungs- und Handchirurgie (Direktor Prof. Dr. med. W. Schneider), Otto-von-Guericke

Universität Magdeburg, Magdeburg, Deutschland

Zielsetzung: Der freie mikrovaskuläre Bauchfettlappen (TRAM / DIEP) stellt eine Standardmethode zur Rekonstruktion der weiblichen Brust nach karzinombedingter Mastektomie oder Ablatio dar. Die Erfahrungen der letzten Jahre haben gezeigt, dass zunehmend auch Patientinnen mit einer störenden Kapselfibrose sowohl nach ästhetisch als auch nach medizinisch indiziertem Brustaufbau mit einem Implantat eine neue Klientel für den freien Bauchhautlappen darstellen. Materialien und Methoden: Zwischen 1999 und 2003 wurden insgesamt 6 Frauen (Alter durchschnittl. 35 a), die nach Implantataufbau bei subkutaner Mastektomie oder aus ästhetischen Gründen eine sicht- und tastbare Kapselverhärtung entwickelten, dauerhaft mit einem freien Bauchfettlappen rekonstruiert. Verwendet wird der freie DIEP oder TRAM. Anschlußgefäße sind der subscapuläre Gefäßbaum. Die Hebestelle wurde mit einer Abdominoplastik verschlossen. Ergebnisse: Komplikationen waren kleine Dog ears an der Hebestelle. Instabilitäten der Bauchwand oder Lappenverluste traten nicht auf. Nach 6-12 Monaten wurde in Einzelfällen eine Straffungsoperation der Brüste durchgeführt. Das subjektive ästhetische Ergebnis bezüglich Tastgefühl, Symmetrie und Natürlichkeit wurde als sehr gut beurteilt. Auch die gleichzeitige Straffung der Bauchdecke wurde vorteilhaft bewertet. Zusammenfassung: Der freie Bauchfettlappen unter Berücksichtigung einer gering höheren Komplikationsrate im Vergleich zur Kapselexzision und Implantatwechsel stellt ein geeignetes Verfahren zur dauerhaften und ästhetisch ansprechenden Rekonstruktion der weiblichen Brust bei störender rezidivierender Kapselfibrose dar.

V4

\section{Ist die axilläre Lymphonodektomie überflüssig?}

* Jutta Engel ${ }^{1}$, Annette Lebeau ${ }^{2}$, Hansjörg Sauer ${ }^{3}$, Dieter Hölzel ${ }^{1}$

${ }^{1}$ Tumorregister des Tumorzentrums München, München,

Deutschland

${ }^{2}$ Pathologisches Institut der LMU München, München, Deutschland ${ }^{3}$ Medizinische Klinik III, Klinikum Großhadern der LMU München, München, Deutschland

Zielsetzung: Zusammenstellung der Argumente für den Verzicht auf die Axilladissektion. Materialien und Methoden: Literaturreview experimenteller und klinischer Studien sowie Daten des Tumorregisters München liefern den Evidenznachweis.

Ergebnisse:

1. Molekularbiologische Untersuchungen zeigen, dass eine Fernmetastasierung durch einen sekundär entstandenen Tumor unwahrscheinlich ist.

2. Genexpressionsanalysen zeigen Grenzen des prognostischen Wertes des Lymphknotenstatus.

3. Der Lymphknotenstatus ist eine Proxyvariable für die Zeitdauer der Tumorzellmigration.

4. Bei einer mehrjährigen Latenzzeit der Fernmetastastasierung können verschiedene Organmetastasen kaum nach dem Kaskadenprinzip entstanden sein.

5. Auch der mikroskopische Lymphknotenbefall ist ein Hinweis auf eine lang andauernde Tumorzellmigration. 
6. In neoadjuvanten Studien ist der Lymphknotenstatus reversibel ohne Survivalreduktion. Eine Filterfunktion der Lymphknoten ist somit unwahrscheinlich

7. Randomisierte Studien belegen, dass weder die Entfernung parasternaler noch axillärer Lymphknoten einen Einfluss auf das Langzeitüberleben haben.

8. Die Bestrahlung parasternaler Lymphknoten bringt keine Verbesserung des Survivals.

9. Es besteht kein Überlebensvorteil bei lateralem vs. medialem Tumorsitz.

10. Die zusätzliche operative oder strahlentherapeutische Therapie de Lymphknoten im Level III ergibt identische Überlebensraten im Vergleich zur Beschränkung auf das Level I oder I und II. Aus der Gleichwertigkeit der Methoden folgt deren Wirkungslosigkeit.

11. In zirka $30 \%$ der Lymphknoten positiven Fälle werden beim heutigen Standard befallene Lymphknoten belassen.

12. Mortalitätsunterschiede zwischen Regionen und Krankenhäusern werden auf die Früherkennung zurückführt und belegen den $\mathrm{Zu}$ sammenhang mit dem Tumordurchmesser.

13. Die Axilladissktion beeinträchtigt die Lebensqualität.

Zusammenfassung: Logisch und empirisch ist der Paradigmenwechsel begründet und wäre ein Gewinn für die Patientinnen.

\section{V5}

\section{Operatives Management der Mammarekonstruktion nach}

\section{Ablatio Mammae}

*Anette Görg, Christian Roessing, Siegfried Grandel

Klinik für Palstische Chirurgie, Hand- und Wiederherstellungs-

chirurgie, Luisenhospital Aachen, Aachen, Deutschland

Das operative Management der Mammarekonstruktion unserer Klinik als Stufenkonzept der Aufwendigkeit des Operationsverfahrens der Rekonstuktion in Relation zum Patientenwunsch unabhängig von histologischer Diagnose und damit Prognose wird vorgestellt. In den letzten Jahren überwogen die prothetischen Rekonstruktionen gegenüber Lappenplastiken. Hierzu werden Vor- und Nachteile der subcutanen oder submuskulären Implantateinlage, die von großer Mehrheit favorisiert wird, vorgestellt. Der prothetischen Rekonstruktion werden die Ergebnisse der Mammarekonstruktion mit gestielten Lappenplastiken (TRAM Flap, Latissimus Flap) gegenüber gestellt und die ersten Erfahrungen mit DIEP Flaps vorgestellt.

\section{V6}

\section{Behandlungsstrategien zur Thoraxwandrekonstruktion aus Plastisch Chirurgischer Sicht}

\section{*Christoph Heitmann, Gisbert Holle, Michael Sauerbier,} Günter Germann

Klinik für Hand-, Plastische und Rekonstruktive ChirurgieSchwerbrandverletztenzentrum, Berufsgenossenschaftliche Unfallklinik Ludwigshafen, Plastische und Handchirurgie der Universität Heidelberg, Deutschland

Ziele der Thoraxwandrekonstruktion sind Wiederherstellung der Thoraxwandstabilität, eine gute Weichteildeckung, sichere Abdeckung der intrathorakalen Strukturen, Erhaltung der Lungenfunktion und ein möglichst ästhetisches Gesamtergebnis. Der Beitrag zur Defektdeckung von Seiten der Plastischen Chirurgie stellt sich in einer Übersicht wie folgt dar:

Kleine Defekte unter $6 \mathrm{~cm}$ im Bereich des Thoraxes können meist suffizient primär oder durch lokale Lappenplastiken verschlossen werden. Mediale Sternumdefekte können bei adipösen Patienten in Ausnahmefällen durch Verschiebung beider Brüste zur Mitte hin erfolgen (Zyklopenbrust). Der mykutane Pektoralis Major Lappen bildet die Primäroption bei Defekten der vorderen, zentralen Brustwand. Defekte am oberen oder lateralen Brustkorb können am ehesten mit einem gestielten Latissimus dorsi versorgt werden. Die größte Variationsbreite bietet der myokutane Rektus- abdominis Lappen, entweder als transversale (TRAM) ode als vertikale (VRAM) Variante. Diese Lappenplastiken lassen sich wie der Latissimus dorsi zur bloßen Defektdeckung aber auch zur Brustrekonstruktion nach Mammaablatio einsetzen. Große intrathorakale
Weichteildefekte können mit der Omentum majus Lappenplastik gefüllt werden. Aufgrund der guten Vaskularisation stellt sie einen idealen Wundgrund für die gleichzeitige Spalthauttransplantation dar. Mikrochirurgische Lappenplastiken sind bei der Primärrekonstruktion im Thoraxbereich selten indiziert und eher Sekundäreingriffen und Rezidiven vorbehalten. Fazit: Die Plastische Chirurgie ist aus modernen onkologischen Konzepten nicht wegzudenken. Das Spektrum der rekonstruktiven Möglichkeiten erlaubt nicht nur eine Deckung auch größerer Defekte, sondern ermöglicht in vielen Fällen auch ein entsprechendes ästhetisches Ergebnis. Auch im Bereich der palliativen Chirurgie sind viele Eingriffe mit unterschiedlicher Zielsetzung erst durch die Einbindung der Plastischen Chirurgie in multi- modale Therapiekonzepte ermöglicht worden.

\section{V7 \\ Die modifizierte B-Technik, ein sicheres onkologisches Verfahren der plastischen brusterhaltenden Chirurgie}

*Gisela Helms, Eugenia Remmel, Rolf Kreienberg Universitätsfrauenklinik UIm, UIm, Deutschland

Zielsetzung: Ursprünglich beschreibt Regnault 1974 mit der B-Technik ein Verfahren zur Mammareduktion. Diese Technik eignet sich auch für die brusterhaltenden Chirurgie beim Mammakarzinom. Es erfolgt die enbloc-Resektion eines radiären Drüsensegmentes mit Haut, anschließend die Rezentralisierung der Areola nach partieller periareolärer Deepithelialisierung. Auf eine Unterminierung der Haut und intramammäre Verschiebelappen wird verzichtet. Das Verfahren ist in jedem Brustquadranten anwendbar. Auch bei relativ ungünstigem Größenverhältnis von Tumor zu Brust kann eine harmonische Brustform erhalten werden. Material und Methoden: Von 2002 bis 3/2004 wurde bei 78 Frauen mit einem erwarteten großen intramammären Defekt 79x die modifizierte B-Technik durchgeführt. Hierbei erschien die Brusterhaltung onkologisch möglich, eine Tumorektomie ließ jedoch ein unbefriedigendes kosmetisches Ergebnis erwarten. Die Umschneidungsfigur und insbesondere die geplante Position der zu rezentrierenden Areola wurden präoperativ bei aufrechter Patientin angezeichnet. Ergebnisse: 75 Frauen hatten ein Malignom (63 primäre Mammakarzinome, 4 Rezidive, 7 DCIS, 1 Melanom), bei 4 Patientinnen war die Histologie benigne. Das mediane Resektionsvolumen betrug $87 \mathrm{~g}$ (7-333 g), die mediane Tumorgröße $30 \mathrm{~mm}$ (7-60 mm). Bei 20 Frauen wurde aufgrund einer R1-Situation eine Nachresektion nötig, 14x war es eine Ablatio mammae, 6x blieb die Brusterhaltung möglich. Postoperativ gab es eine Wundinfektion. Zusammenfassung: Die modifizierte B-Technik eignet sich für die Tumorchirurgie der Brust. Sie ist ein onkologisch sicheres Verfahren, weil ein repräsentatives Drüsenvolumen unter Darstellung klarer Resektionsgrenzen entfernt wird. Die Zahl der Nachresektionen zur abschließenden Brusterhaltung ist gering. Eine harmonische Brustform kann auch bei relativ großen Resektionsvolumina unabhängig von der Brustgröße erhalten werden. Eine präzise präoperative Planung ist Voraussetzung.

\section{V8 \\ Stellenwert und Möglichkeiten der operativen Therapie der radiogenen Plexitis}

\section{Robert Hierner}

Plastische, Rekonstruktive und Ästhetische Chirurgie,

Universitätsklinikum Gasthuisberg, Katholische Universität Leuven, Leuven, Belgien

Bei der radiogenen Plexitis handelt es sich um einen chronischen Prozeß mit ständiger, schubweiser Symptomaggravation. Klinische Leitsymptome sind sensible (Parästhesien, Dysästhesien, Anästhesien) und motorische Ausfälle (Parese, Paralyse) und Schmerzen. Da diese Beschwerden auch Ausdruck eines Tumorwachstums (Rezidiv oder primär strahleninduziert) sein können, muß eine Neoplasie im Bereich des Plexus brachialis abgeklärt werden.

Eine kausale Therapie der radiogenen Plexitis gibt es nicht. Ziel der symptomatischen Therapie ist eine möglichst lange Stabilisierung, vor allem der Schmerzen, auf einem erträglichen Beschwerdeniveau. Motroische Ausfälle sind mit Ausnahme der proximalen Muskeln meist irreversibel. Die Wahl des therapeutischen Vorgehens ist abhängig von 1) Art und 
Stadium der Tumorerkrankung (pTNM), 2) Allgemeinzustand und Alters des Patienten, 3) den zusätzlich bestehenden Strahlenschäden im Bereich von Haut und/oder Knochen, 4) dem klinischen Stadium der Plexitis (v.a Vorhandensein von Schmerzen), und 5) den intraoperativ gefundenen morphologischen Veränderungen. Die konservative Therapie stellt die Basis jeder Behandlung dar.

Die operative Revision des Plexus brachialis mit externe Neurolyse/Epineurotomie + vaskularisierter Gewebetransfer ist heutzutage nur bei wenigen definierten Situationen indiziert und zu empfehlen. Wegen der möglichen schwerwiegenden Komplikationen und der hohen Rate an sekundärer Fibrosierung des Omentum majus, sollten Muskel- oder Muskel-Haut-Lappenplastiken bevorzugt eingesetzt werden.

\section{V9}

Narbenunabhängige Rekonstruktion der Brustwarze mit zwei Transpositionsläppchen

\section{Josef Hoch}

Klinik für Hand-, Brust- und Plastische Chirurgie,

Klinikum Neustadt in Holstein, Neustadt in Holstein, Deutschland

Zielsetzung: Die Schaffung einer dauerhaften Projektion stellt das Hauptproblem bei der Brustwarzenrekonstruktion dar und bleibt trotz zahlreicher und von der Idee her erfolgversprechender Techniken eine plastischchirurgische Herausforderung, insbesondere wenn Narben das Rekonstruktionsfeld kreuzen. Methodik: Zur Lösung dieses Problems werden parallel zum Narbenverlauf zwei einander gegenüberliegende, ortsständige Transpositionsläppchen geplant, deren Blutzufuhr nicht durch die Narbe limitiert wird. Durch Positionierung der transponierten Läppchen auf einer korialen Auflagefläche und durch eine konsequente postoperative Vertikalisierung mit einem «Sektkorkenhalter aus Draht» wird die Papilla mammae stabilisiert und die Mamillenspitze sinkt nicht in die Ebene der rekonstruierten Areola ein. Die relativ einfache und sichere Technik, die auch in örtlicher Betäubung durchführbar ist, wird ausführlich demonstriert und anhand von Fallbeispielen dargestellt.

V10

\section{Komplikationen nach Mamma-Reduktionsplastik mit vertikaler} und horizontal-vertikaler Narbe im Vergleich

\section{*Eugen Hoefter, Oliver Theusinger, Charlotte Holm,} Wolfgang Mühlbauer, Milomir Ninkovic

Abteilung für Plastische, Rekonstruktive und Handchirurgie, Zentrum für Schwerbrandverletzte

Klinikum München-Bogenhausen der Technischen Universität München, München, Deutschland

Ziel der Studie war die Analyse der Komplikationen nach MammaReduktionsplastik mit vertikaler gegenüber horizontal-vertikaler Narbe im Vergleich. Material und Methoden: 165 Patienten wurden im Zeitraum 1999 bis 2001 retrospektiv ausgewertet. Bei 57 Patienten wurde eine Reduktion mit vertikaler Narbe durchgeführt (Gruppe I)gegenüber $108 \mathrm{~Pa}$ tienten mit konventioneller horizontal-vertikaler Narbe und kranial gestielten Mammillen (Gruppe II). Parameter wie Alter, Body Mass Index (BMI), Resektionsgewicht sowie alle Früh- und Spät-Komplikationen wurden erfaßt. Die statistische Auswertung erfolgte mit dem T-Test nach Mann-Whitney und dem Chi-Quadrat-Test (Pearson). Ergebnisse: Die untersuchten Gruppen zeigten keine signifikanten Unterschiede hinsichtlich Alter (34,1 vs. 37,9 Jahre), BMI (24,8 vs. $\left.25,8 \mathrm{~kg} / \mathrm{m}^{2}\right)$. und mittleres Resekionsgewicht (553,5 vs. 552 g) je Brust. Erfasst wurden frühe Komplikationen während des stationären Aufenthaltes wie Hämatom, Infektion, partielle Mammillen-Nekrose, Nekrose des Drüsenkörpers sowie Wunddehiszenz. Späte Komplikationen umfassten hypertrophe Narben und dog ears. Die Komplikationsrate in Gruppe I mit vertikaler Narbe betrug $43,9 \%$ (25 von 57 Patienten) gegenüber 40,7\% (44 von 108 Patienten) in Gruppe II mit horizontal-vertikaler Narbe. Die etwas höhere Komplikationsrate der Technik mit vertikaler Narbe im Vergleich zur konventionellen Methode war statistisch nicht signifikant. Zusammenfassung: Die Technik der Mamma-Reduktion mit vertikaler Narbe hat gegenüber der konventionellen Technik mit umgekehrter T-Narbe in unserer Klinik keine signifikant erhöhte Komplikationsrate. Zusätzliche Me- thoden wie eine adjuvante Liposuktion und großflächige Unterminierung der Hautlappen wurden bei Anwendung der vertikalen Technik strikt vermieden, da sie die Komplikationsrate nachweislich erhöhen. In jedem Fall muß die Patientin über die nicht unerhebliche Komplikationsrate bei beiden Methoden unterrichtet werden und sollte darüber aufgeklärt sein.

\section{V11 \\ Tief oder Oberflächlich? - Unterschiede in den Perfusionszonen von DIEP-und SIEA-Lappen}

${ }^{*}$ C. Holm, M. Mayr, E. Höfter, U. J. Pfeiffer, M. Ninkovic Abt. für Plastische Chirurgie, Zentrum für Schwerbrandverletzte, Klinikum Bogenhausen, München, Deutschland

Einleitung: Die Perfusionszonen des TRAM-Lappens wurden von Carl Hartrampf beschrieben. Sie stellen die Basis für die Brustrekonstruktion dar, wobei Zone III und IV fallweise verworfen werden. Hartrampfs Zoneneinteilung beruhte auf dem klinischen Eindruck der Durchblutung eines einseitig gestielten TRAM-Lappens mit der Basis an den superioren epigastrischen Gefäßen. Heute hat die freie mikrovaskuläre Gewebeübertragung von dem Unterbauch den gestielten Transfer abgelöst. Sie basiert auf dem inferioren tiefen und oberflächlichen epigastrischen System (TRAM, DIEP oder SIEA). Ob die Hartrampfsche Zoneneinteilung, die kritiklos für die freie Gewebeübertragung übernommen worden ist, für diese Lappen auch Gültigkeit hat, oder ob die unterschiedlichen Gefäßachsen zu einem unterschiedlichen Durchblutungsmuster führen, ist bisher nie untersucht worden. Ziel der Studie: Quantitative Analyse der Perfusionszonen des freien DIEP und SIEA Lappens. Methode: Im Rahmen einer klinischen prospektiven Studie wurde die Durchblutung freier Unterbauchlappen, die auf dem tiefen oder oberflächlichen epigastrischen System basierten, mittels laser-induzierte Fluoreszenz von Indozyaningrün gemessen. Die Hautellipse wurde intraoperativ nach Hartrampf eingeteilt und nummeriert. Der Anstieg der Fluoreszenz wurde für jede Zone aufgezeichnet und mit der Intensitätskurve von normalem Gewebe (Brustwand) verglichen. Material: Untersucht wurden 30 Patienten, bei denen eine Brustrekonstruktion mit einem freien Unterbauchlappen durchgeführt wurde. Es wurden insgesamt 20 DIEP und 10 SIEA-Lappen untersucht. Ergebnisse: DIEP-Lappen: Die Ergebnisse der Perfusographie zeigten eine fortschreitende, stufenweise Durchblutung benachbarter Haut durch das tiefe epigastrische System. Die Perfusion in Zone III war signifikant besser als in Zone III $(\mathrm{p}<0,05)$. Die Perfusion in Zone IV war extrem variabel und reichte von sehr schlecht ( $0 \%$ Perfusionsindex) bis ausgezeichnet (55\% Perfusionsindex). SIEA-Lappen: Die Perfusion des SIEA-Lappens war auf der ipsilateralen Seite sehr gut, aber die Gefäßverbindungen über die Mittellinie schienen beim oberflächlichen System signifikant weniger entwickelt im Vergleich zum tiefem epigastrischen System. Schlussfolgerungen: Die klassische Hartrampf Zoneneinteilung ist für den freien mikrovaskulären Gewebetransfer (gestielt am inferioren epigastrischen System) nicht mehr gültig. Aufgrund der Perfusionsmessungen schlagen wir einen Austausch der Zonen II und III vor. Die oberflächliche Gefäßachse des SIEA-Lappens führt zu einer unterschiedlichen Durchblutungscharakteristik als beim DIEP-Lappen. Die Durchblutung der kontralateralen Seite scheint vor allem viel weniger zuverlässig als für den konventionellen DIEP-Lappen.

\section{V12}

\section{Das MCDBP (multi center database project) - ein update}

${ }^{*}$ Peter Konstantiniuk ${ }^{1}$, Anton Haid ${ }^{2}$, Christian Peters-Engl ${ }^{3}$, Christoph Rageth ${ }^{4}$, Ursula Selim ${ }^{5}$

${ }^{1}$ Univ. Klinik für Chirurgie, Graz, Österreich

${ }^{2}$ Landeskrankenhaus, Feldkirch, Österreich

${ }^{3}$ Krankenhaus Lainz, Wien, Österreich

${ }^{4}$ Brustzentrum, Zürich, Schweiz

${ }^{5}$ Hanuschkrankenhaus, Wien, Österreich

Hintergrund: Eines der größten Probleme multizentrischer Studien ist die Zusammenführung der Daten. Ziel dieses Projektes ist die Errichtung eines dafür geeigneten Instrumentes, wobei als Pilotprojekt das Mammakarzinom ausgewählt wurde. Patientinnen und Methode: Unter Verwendung von ACCESS 97 wurde eine Mamma-Datenbank errichtet. Die 
Zusammenführung der Daten erfolgte bisher via e-mail, heuer wird auf eine internetbasierte Version umgestellt. Ergebnisse: Bis heute wurden 6827 Datensätze von 30 verschiedenen Abteilungen aus Österreich und der Schweiz in dieser Datenbank gespeichert. Schlußfolgerung: Nach bisherigen Erfahrungen scheint das Grundkonzept erfolgreich und die Datenbank ein wertvolles Instrument zu sein.

\section{V13}

\section{Thoraxwandrekonstruktion mit regionalen und freien} Lappenplastiken nach ausgedehnten Resektionen

\section{*Stefan Thomas Krischak, Jörg Borges, Eva Maria Lang,} Gerhard Björn Stark

Plastische und Handchirurgie, Freiburg, Deutschland

Zielsetzung: In unserer Abteilung wurden zahlreiche regionale und freie Lappenplastiken zur Deckung von Thoraxwanddefekten durchgeführt. Im Rahmen einer retrospektiven Untersuchung wurde ein Algorithmus zur Rekonstruktion ausgedehnter Thoraxwanddefekte erstellt. Material und Methoden: Wir untersuchten retrospektiv 70 Patienten, mit ausgedehnten Thoraxwanddefekten hinsichtlich Diagnose, Art der Lappenplastik, Komplikationen und spezielle Indikationen für freie Lappenplastiken. Ergebnisse: Die Indikationen waren Strahlenschäden $(\mathrm{n}=12)$ und ausgedehnte Infektionen im Bereich des Brustkorbes $(\mathrm{n}=10)$ und vor allem maligne Tumore insbesondere der Brust, hiervon 24 Patienten mit ausgedehnten Primärtumoren, 22 Patienten mit lokalen Rezidiven und $2 \mathrm{~Pa}-$ tienten mit Metastasen. Bei 28 Patienten verwendeten wir den M. latissimus, bei 19 den M. pectoralis major als und bei 7 den M. rectus abdominis als regionale Lappen. Bei 6 Patienten konnte eine Deckung mit lokalen Verschiebelappenplastiken durchgeführt werden. Bei 10 Patienten erfolg te die Deckung mit freien Lappen. Hierbei wurde bei 7 Patienten die Rekonstruktion ein TRAM-Lappen, sowie ein freier Latissimus-Lappen verwendet. Bei zwei Patienten mit ausgedehnten Tumoren im schulternahen Brustwandbereich erfolgte eine Exartikulation im Schultergelenk Der entstandene Defekt konnte mit frei transplantiertem Gewebe von den distalen Amputaten gedeckt werden. Alloplastische Materialien wurden bei 5 Patienten verwendet. Schwere Komplikationen wurden be keinem Patienten beobachtet, insbesondere kein Lappenverlust. Als häufigste Komplikationen wurden lokale Wundheilungsstörungen und ein Serom dokumentiert. Schlussfolgerung: Für die Deckung von ausgedehnten Thoraxwanddefekten sind sowohl regionale als auch freie Lappen eine sichere Therapieoption. Basierend auf diesen Daten wurde ein Algorithmus für die Thoraxwandrekonstruktion erstellt. Insbesondere bei erforderlicher Amputation kann das Amputat als Spender für einen freien Lappen dienen.

\section{V14}

\section{Gynäkomastie - Fehler, Komplikationen und Lösungen}

${ }^{*}$ Anette Meiré, Dirk F. Richter, Mathias Reichenberger, Alexander Stoff, Michael Schneeweiß, Maria Achontaki

Abteilung für Plastische Chirurgie - Dreifaltigkeits-Krankenhaus, Wesseling, Deutschland

Für die operative Therapie der Gynäkomastie steht eine Vielzahl unter schiedlicher Behandlungen zur Verfügung. Diese richten sich nach dem klinischen Befund und dem Anteil an Fett- bzw. Drüsengewebe. Während bei der lipomatösen Gynäkomastie ohne Drüsenhypertrophie eine allei nige Liposuktion ausreicht, empfiehlt sich bei der gemischten Form eine Kombination aus Liposuktion und offener Resektion mit periareolärem Rundschnitt. Fehlermöglichkeiten und Komplikationen treten bei ungünstiger Schnittführung auf, was zu prominenten und entstellenden Narben führt. Alleinige Entfernung des Drüsengewebes resultiert in einer Dellenbildung im Areolenbereich, ebenso kann die exzessive Resektion zu Einsenkungen oder Adhärenzen im Operationsgebiet führen. Fehlende Retraktion und Schrumpfung der Haut imponieren als Hautüberschuss. Anhand ausgewählter Beispiele soll eine Behandlungsstrategie zur Vermeidung solcher Fehler bei Primäreingriffen erläutert werden. Weitere Sekundäreingriffe bei der Gynäkomastie zeigen, dass zahlreiche aus der Brustchirurgie der Frau bekannte Verfahren auch beim Mann Anwendung finden. Zur Korrektur dieser Komplikationen wurden in unserer
Klinik modifizierte Bruststraffungen nach Ribero, Strömbeck oder Pitanguy durchgeführt. Die Verwendung von Turn-over-Flaps erwies sich vor allem bei der Korrektur von Verziehungen oder Adhärenzen als erfolgreich.

\section{V15}

\section{Darstellung der Fehlerrate und Analyse der Fehler nach 1000 Sentinellymphknotenbiopsien und Sentinellymph- knotenbiopsieversuchen}

${ }^{*}$ Roland Reitsamer ${ }^{1}$, Florentia Peintinger ${ }^{2}$, Christian Menzel ${ }^{1}$, Walter Cimpoca ${ }^{1}$, Lukas Rettenbacher ${ }^{3}$, Eva Prokop ${ }^{4}$

${ }^{1}$ Universitätsklinik für Spezielle Gynäkologie,

Paracelsus Medizinische Privatuniversität, Salzburg, Österreich ${ }^{2}$ Abteilung für Gynäkologie, Landeskrankenhaus, Leoben,

Österreich

${ }^{3}$ Universitätsklinik für Nuklearmedizin, Paracelsus Medizinische Privatuniversität, Salzburg, Österreich

${ }^{4}$ Pathologisches Institut der Paracelsus Medizinischen

Privatuniversität, Salzburg, Österreich

Die Sentinellymphknotenbiopsie (SLNB) hat sich wie kaum eine andere chirurgische Innovation, vor Vorliegen von prospektiv randomisierten Daten, einen festen Platz in der operativen Therapie des Mammakarzinoms erobert. An den meisten Brustzentren wird die SLNB bei Sentinellymphknoten (SLN) negativen Patientinnen als Alternative zur axillären Lymphknotendissektion (ALND) angeboten. An der Universitätsklinik für Spezielle Gynäkologie der Paracelsus Medizinischen Privatuniversität Salzburg wird die SLNB seit März 1998 durchgeführt. Seit Beginn wird zur Identifikation des SLN die kombinierte Methode mit Injektion von Technetium 99m-markiertem Albumin und Injektion von blauem Farbstoff durchgeführt. Nach nun erfolgten 1000 Sentinellymphknotenbiopsien und Sentinellymphknotenbiopsieversuchen wird eine Analyse der Fehler durchgeführt. Es werden sowohl die Fälle von technisch nicht darstellbaren SLNs, die vom Operateur nicht aufgefundenen SLNs, als auch die falsch negativen SLNBs analysiert, und es wird versucht die Fehlerquelle zu finden. In $10 \%$ der 1000 Fälle konnte der SLN entweder nicht dargestellt werden, nicht aufgefunden werden, oder war falsch negativ. Aus diesen Daten kann auch die Frage der SLNB nach neoadjuvanter Chemotherapie oder der SLNB bei multizentrischen/multifokalen Mammakarzinomen neuerlich diskutiert werden. Es wird versucht die Indikations-/Kontraindikationsliste zur SLNB neu zu erstellen.

\section{V16}

\section{Der Einfluss der intraduktalen Komponente auf den} intramammären Tumorrest des Mammakarzinoms

*Mandy Renz' ${ }^{1}$ Andreas Plötner ${ }^{2}$, Dagmar Langanke', Uwe Köhler ${ }^{2}$ ${ }^{1}$ St. Elisabeth-Krankenhaus, Leipzig, Deutschland ${ }^{2}$ Städtisches Klinikum St. Georg, Leipzig, Deutschland

Zielsetzung: Das Vorhandensein einer extensiven intraduktalen Komponente (EIC) gilt als ein Risikofaktor für das Auftreten eines Lokalrezidivs. Das Ziel der Arbeit bestand darin, den Zusammenhang zwischen dem Vorhandensein und der Ausdehnung der intraduktalen Komponente beim invasiv duktalen Karzinom und dem verbliebenen Tumorrest nach Excision des Karzinoms zu belegen. Materialien und Methoden: Wir untersuchten 230 Patientinnen retrospektiv, die zwischen 1998 und 1999 im Städtischen Klinikum St. Georg in Leipzig primär brusterhaltend operiert oder mastektomiert worden waren. Die 148 invasiv duktalen Karzinome wurden auf das Vorhandensein einer intraduktalen Komponente (EIC/PIC) mit Angabe der Ausdehnung überprüft und nachklassifiziert. Ein invasiver und/oder noninvasiver Tumoranteil im Nachresektat bzw. Mastektomiepräparat wurde als positiver Rest registriert. Ergebnisse: Von den Patientinnen mit einer PIC/EIC hatten 52\% einen invasiven und/oder noninvasiven Rest in der verbliebenen Brust. Im Gegensatz dazu wurden bei den Frauen mit einer MIC in 34,9\%, und bei Fehlen der intraduktalen Komponente in 9,1\% ein Tumorrest im Nachresektat bzw. Mastektomiepräparat nachgewiesen. Bei einem Viertel der Fälle mit PIC/EIC trat das Karzinom multizentrisch auf, dagegen nur in $8,4 \%$ der Karzinome mit MIC und bei keinem Karzinom ohne intraduktale Kom- 
ponente. Zusammenfassung: Der Tumorrest ist mit dem Vorhandensein einer intraduktalen Komponente beim Mammakarzinom erhöht. Trotz einer angestrebten BET-Rate von $60-70 \%$ muss die Entschedung zur brusterhaltenden Operation in diesen Fällen unter sorgfältiger und individueller Risiko-Nutzen-Abwägung getroffen werden.

\section{V17}

\section{Immediate Breast Reconstruction after Skin-Sparing Mastectomy with Autogenous Tissue}

${ }^{*}$ Dirk J. Schaefer ${ }^{1}$, Mirjam Schlatter ${ }^{1}$, Martin Haug ${ }^{1}$, Oliver Scheufler ${ }^{2}$, Walter R. Marti ${ }^{2}$, Daniel Oertli ${ }^{2}$, Gerhard Pierer ${ }^{1}$ ${ }^{1}$ Departement of Plastic and Reconstructive Surgery, Basel, Switzerland

${ }^{2}$ Departement of General Surgery, University Hospital, Basel, Switzerland

Skin-sparing mastectomy describes the procedure of mastectomy with a minimum amount of skin excision which includes the nipple-areola complex and biopsy sites. Immediate breast reconstruction with autogenous tissue can recreate a breast mound that closely resembles the native breast in shape and consistency.

17 patients with early-stage breast cancer (DCIS - ductal carcinoma in situ) and two patients with bilateral disease underwent skin-sparing mastectomy and immediate autogenous reconstruction in a two years time period and were closely followed.

Depending on the needed breast volume a pedicled latissimus dorsi flap was chosen in 10 cases or a microvascular tissue transfer (3 TRAM and 8 DIEP flaps) was carried out. The reconstruction of the nipple-areola complex followed in a second step three months later. The women's opinion concerning the satisfaction with their reconstructed breast was evaluated using a questionnaire.

Among these patients (two women with bilateral reconstructions) no instances of flap loss or local recurrence were observed. Complications at the donor site were minor and limited to delayed wound healing or seroma. The aesthetic results were judged between good to excellent because the reconstructed breasts resembled the native breast in shape and consistency. Our findings support the assumption the technique of this procedure being a safe one. However, an appropriate patient selection and technical expertise in performing the mastectomy and reconstruction is considered essential. The preservation of the original skin envelope facilitates immediate breast reconstruction and enables excellent and natural results.

\section{V18}

\section{Die ausgeprägte Gynäkomastie - eine kosmetische Herausforderung}

${ }^{*}$ Christian Schmidt, Jan Zinndorf

Klinikum Offenburg, Fachbereich für Plastische, Ästhetische und Wiederherstellungschirurgie, Offenburg, Deutschland

Die Gynäkomastie des Mannes stellt mit einer Inzidenz von 32 bis $65 \%$ der männlichen Population die häufigste gutartige Brustveränderung be Männern dar. Sie kommt primär idiopathisch, v. a. in der Pubertät vor, se kundär bei Erkrankungen z.B. der Keimdrüsen und als medikamentöse Nebenwirkung.

Die Indikation zur operativen Entfernung bzw. Korrektur der Gynäkomastie ist nicht nur kosmetisch aufgrund der psychosozialen Belastung der Patienten, sondern auch medizinisch begründet. Ein Mammacarcinom muß insbesondere bei rasch wachsenden und einseitigen Vergrößerungen ausgeschlossen werden.

Anhand eines Beispieles wird die persönliche Operationstechnik des Autors zur Korrektur ausgeprägter Gynäkomastien in Verbindung mi Areolenhypertrophie vorgestellt: Die Mamille wird auf das endgültige $\mathrm{Maß}$ ringförmig umschnitten, ein individuell angepasster Hautring wird als neues Mamillenlager deepithelisiert. Von kaudal her wird die hypertrophe Brustdrüse mit dem umliegenden Fettgewebe entfernt. Der MAK bleibt kranial gestielt und kann formend eingepasst werden. Über den kaudalen Zugang wird noch vor dem endgültigen Wundverschluß feinmodellierend überschüssiges Fett abgesaugt. Es resultiert eine kosmetisch unauffällige periareoläre Narbe.
Fazit:Mit der hier vorgestellten Methode lassen sich auch sehr große Gynäkomastien mit entsprechend großer Mamille auf sichere und einfache Weise kosmetisch günstig anpassen. Im Gegensatz zur reinen Fettabsaugung kann so auch Drüsengewebe zur histopathologischen Begutachtung gewonnen werden.

V19

Technik und Langzeitergebnisse des modifizierten skate-flap zur Rekonstruktion des nipple-areola-Komplexes

*Barbara Schroer, Thomas Lange, Michael Kaun, Peter Mailänder, Hans-Günther Machens

Plastische und Handchirurgie, Zentrum für Schwerbrandverletzte, Universitätsklinikum Schleswig-Holstein/Campus Lübeck, Lübeck, Deutschland

Einleitung: Bei der Rekonstruktion des nipple-areola-Komplexes ist der Erhalt einer dauerhaften Projektion der Neo-Brustwarze das Hauptproblem. Gründe dafür sind oft die primär unsichere Durchblutung der Lappenanteile und der daraus resultierende Gewebeverlust mit Vernarbung. Die hier gezeigte Modifikation des skate-flap ermöglicht auch bei schwierigen lokalen Gewebeverhältnissen eine sichere Perfusion der verwendeten Lappen. Im Langzeitverlauf weist die Neo-Brustwarze bei dieser Technik nur geringe Verluste an Volumen und Projektion auf. Material und Methoden: Seit 1997 führten wir bei 6 Patientinnen zwischen 36 und 64 Jahren eine Rekonstruktion des nipple-areola-Komplexes mit dieser Technik durch. In allen Fällen war eine Mammaablatio bei Mamma-Ca vorausgegangen und eine Brustrekonstruktion durch gestielten myokutanen M. Latissimus-Dorsi-Lappen durchgeführt worden. In 4 Fällen hatte die Projektion der Mamille komplett im Bereich der Monitorinsel gelegen. Zweimal mußte autochthone Haut in den cranialen Mamillenanteil einbezogen werden. Der Warzenvorhof wurde in allen Fällen durch Vollhaut aus der Oberschenkelinnenseite, die Brustwarze selbst aus einem modifizierten bilobed skate-flap mit zentralem Gefäßstiel rekonstruiert. Ergebnisse: Bei allen Patientinnen heilten die rekonstruierten Brustwarzen komplikationslos ein. In einem Falle kam es zu einer Sekundärheilung der Warzenvorhofregion, die aber keine Korrekturoperation erforderte. Die Projektion der Brustwarze maß direkt postoperativ $10 \pm 2 \mathrm{~mm}$ und 12 bis 69 Monate nach der Operation $8 \pm 2 \mathrm{~mm}$. Bei einer Patientin wurde 17 Monate nach Rekonstruktion eine operative Verkleinerung des Brustwarzendurchmessers vorgenommen. Alle Patientinnen waren 12 bis 69 Monate nach der Operation mit dem operativen Ergebnis sehr zufrieden. Schlussfolgerung: Der modifizierte bilobed skate-flap mit zentralem Gefäßstiel stellt eine verlässliche Operationsmethode zur Rekonstruktion eines primär vaskularisierten nipple-areola-Komplexes mit gutem Projektionsergebnis im Langzeitverlauf dar.

\section{V20}

\section{Sentinel-Lymph-Node-Biopsie nach primär systemischer Therapie: Eine Analyse zur Methode und Sicherheit}

* Joke Tio 1,2, Michael Buehner ${ }^{1}$, Heidi Parchent ${ }^{1}$, Pia Wülfing ${ }^{2}$, Ludwig Kiesel2, Heidrun Volkholz ${ }^{3}$, Wolfgang Schulze ${ }^{4}$ Augustinus Harjanto Tulusan ${ }^{1}$

${ }^{1}$ Klinikum Bayreuth, Frauenklinik, Bayreuth, Deutschland 2Universitätsklinikum Münster, Klinik und Poliklinik für Frauenheilkunde und Geburtshilfe, Münster, Deutschland ${ }^{3}$ Klinikum Bayreuth, Institut für Pathologie, Bayreuth, Deutschland ${ }^{4}$ Klinikum Bayreuth, Institut für Strahlentherapie und Nuklearmedizin, Bayreuth, Deutschland

Einleitung: Die Sentinel-Lymph-Node-Biopsie (SLNB) ist eine anerkannte Methode zur Bestimmung des axillären Lymphknotenstatus. Da die primär systemische Therapie (PST) immer häufiger beim lokal fortgeschrittenen Mammakarzinom eingesetzt wird, stellt sich die Frage, ob eine SLNB auch nach PST aussagekräftig ist. Zusätzlich soll untersucht werden, welche Methode der Markierung hierzu am besten geeignet ist. Material und Methoden: Es wurden 88 Patientinnen (medianes Alter: 50 Jahre) nach PST (anthrazyklinhaltige und/oder taxanhaltige Chemotherapie oder Anastrozol) in die Studie eingeschlossen. Zur Identifizierung des Sentinel-Lymph-Nodes (SLN) wurden allen Patientinnen peritumoral 
$2 \mathrm{ml}$ Patentblau-Lösung injiziert, bei 28 Patientinnen wurde zusätzlich 99m-Tc-Nanokoll appliziert. Nach SLNB erfolgte anschließend die konventionelle Axilladissektion (mediane Lymphknoten-Zahl: 16). Ergebnisse: In 83/88 Fällen (93,2\%) konnte der SLN (mediane SLN-Zahl: 2) identifiziert werden. Wurde die alleinige Blau-Methode angewandt, konnte bei 54/60 Patientinnen (90\%) der SLN detektiert werden, wurden beide Methoden angewandt konnte eine Detektionsrate von 100\% (28/28) verzeichnet werden. Es zeigten sich insgesamt 2 Falsch-negative Fälle (Falsch-Negativ-Rate (FNR) insgesamt: 5,7\%), ein Fall in der Gruppe der alleinigen Farstoffmethode (FNR: 4,5\%) und ein Fall in der Gruppe der kombinierten Markierungmethode (FNR: 7,7\%). Schlussfolgerung: Die SLNB nach PST ist ebenfalls eine adäquate Methode zur Bestimmung des axillären Lymphknoten Status. Zur beseren Detektion des SLN sollten jedoch beide Methoden (Patenblau und 99m-Tc-Nanokoll) angewandt werden.

\section{V21}

\section{Sentinel-Lymph-Node-Biopsie beim DCIS}

*Joke Tio 1, 2, Michael Bühner ${ }^{1}$, Heidi Parchent ${ }^{1}$, Nina Rinas ${ }^{1}$ Heidrun Volkholz ${ }^{3}$, Pia Wülfing' ${ }^{2}$ Ludwig Kiesel' ${ }^{2}$, Augustinus Harjanto Tulusan

${ }^{1}$ Klinikum Bayreuth, Frauenklinik, Bayreuth, Deutschland ${ }^{2}$ Universitätsklinikum Münster, Klinik und Poliklinik für

Frauenheilkunde und Geburtshilfe, Münster, Deutschland

${ }^{3}$ Klinikum Bayreuth, Institut für Pathologie, Bayreuth, Deutschland

Einleitung: Die Sentinel-Lymph-Node-Biopsie ist eine anerkannte Methode zur Bestimmung des axillären Status beim invasiven Mammakarzinom. Durch immer besser werdende Diagnostik, werden präinvasive und invasive Mammabefunde in früheren Stadien diagnostiziert. Aus der Literatur ist bekannt, dass Patientinnen mit ausgedehntem DCIS und high grade-DCIS ein höheres Risiko einer Invasion haben. Welche Bedeutung die SLNB für solche DCIS-Fälle haben, sind bisher noch wenig untersucht. Material/Methoden: Eingeschlossen wurden 23 Patientinnen be denen mittels stereotaktischer Vakuumstanze ein DCIS diagnostiziert wurde. In der Mammographie war bei allen Patientinnen suspekter Mikrokalk größerer Ausdehnung aufgefallen. Nach radiologischer Markierung wurde bei diesen Patientinnen die Segmentale-resektion mit Sen tinel-Lymph-Node-Biopsie durchgeführt. Hierzu wurde intraoperativ $2 \mathrm{~m}$ Patentblau-Lösung in die Wundhöhle injiziert. In 6 Fällen wurde zusätzlich am Vortag der Operation 99m-Tc-Nanokoll appliziert. Die SLNB wurde jeweils von erfahrenen Operateuren durchgeführt. Ergebnisse: In 22/23 (95,7\%) Fällen konnte der Sentinel-Lymph-Node (SLN) identifiziert werden. In einem Fall $(4,5 \%)$ war der SLN metastatisch befallen. Eine komplette axilläre Dissektion (insgesamt 14 Lymphknoten) wurde anschließend durchgeführt. In der endgültigen Histologie konnte hierbe ein invasives Carcinom (pT1b) diagnostiziert werden. Außer dem SLN waren keine weiteren Lymphknoten befallen. In zwei weiteren Fällen zeigte sich in der endgültigen Histologie ebenfalls ein invasives Carcinom, jeweils im Tumorstadium pT1b. Ein mikroinvasives Carcinom konnte in einem Fall nachgewiesen werden. Auf eine komplette Axilladissektion wurde in allen 3 Fällen bei negativem SLN verzichtet. Schlussfolgerung: Eine SLNB beim DCIS ist sinnvoll. Hierdurch kann den Patientinnen eine weitere Operation erspart werden. Zumindest sollte eine SLNB bei ausgedehntem Mikrokalk in der Bildgebung und high-grade-DCIS in der Histologie durchgeführt werden.

\section{V22}

\section{Katastrophale Ergebnisse nach Mammarekonstruktion}

*Nestor Torio-Padron, Jörg Borges, Gunter Felmerer, Arash Momeni, G. Björn Stark

Abteilung für Plastische und Handchirurgie, Universitätsklinikum

Freiburg, Freiburg, Deutschland

Indikationen für plastisch-chirurgische Rekonstruktionen beinhalten ästhetische Wiederherstellung und Defektdeckungen bei ausgedehnten Befunden, Lokalrezidiven und Strahlenschäden. Hierzu steht eine Palette an Techniken zur Verfügung, aus welcher individuell nach den Bedürfnissen ausgewählt werden sollte. In einer universitären plastisch-chirur- gischen Fachabteilung am Mammazentrum wurde im Zeitraum von 1993-2004 eine große Bandbreite an Ergebnissen nach fehlgeschlagenen Rekonstruktionen gesehen:

- Schwere Dystrophien, Kontrakturen und Infektionen nach Prothesenoder Expanderimplantation

- Ausgedehnte Nekrosen und Folgezustände an der Brustwand wie auch Entnahmenstelle nach versuchten teilweise historischen lokalen und regionären Lappenplastiken

In vielen Fällen kam es zu monatelangen Verzögerungen bis die Patienten eine adäquate und differenzierte plastisch-chirurgische Behandlung erhielten.

Die plastisch-chirurgische Rekonstruktion beim Mammakarzinom sollte das gesamte komplexe Behandlungsspektrum zur differentiellen Indikationsstellung, Patientenaufklärung und zur Behandlung möglicher Komplikationen beinhalten. Die ausschliessliche Beherrschung isolierter Operationsverfahren für alle Patientinnen beeinträchtigt die Sicherheit und Ergebnisse. Eine fachärztliche plastisch-chirurgische Einheit sollte selbstvertändlicher Bestandteil jedes Mammazentrums entsprechend internationaller Standards sein.

\section{V23}

\section{Rezidivraten beim DCIS: Übersicht der Literatur und Vorstellung eigener Langzeitergebnisse}

*U. von Fritschen, B. Baican, H. Kaisers, K. Exner

Klinik für Plastische Chirurgie, Wiederherstellungs-und

Handchirurgie Markus-Krankenhaus, Frankfurt, Deutschland

Problemstellung: Die Therapie des DCIS ist bis heute nicht standardisiert. Bei suffizienter Primärtherapie ist die Erkrankung heilbar, ein Lokalrezidiv verschlechtert die Prognose jedoch erheblich. Rezidive können bei alleiniger lokaler Resektion in bis $\mathrm{zu} 43 \%$ der Fälle auftreten. Bei ausschließlicher Biopsie muß in bis zu $62 \%$ der Fälle damit gerechnet werden. Der Anteil der invasiven Karzinome an den Rezidivtumoren beträgt zirka $50 \%$. Deshalb wird bei Vorliegen von Risikofaktoren zunehmend die Mastektomie empfohlen. Welche Patientinnen mit alleiniger lokaler Exzision behandelt werden können, ist unklar. Zur Vermeidung von unnötigem Organverlust wird alternativ die Skinsparing Mastektomie (SSM) propagiert. Patienten und Methode: Von 1979-1990 wurden 515 Patientinnen mit Mammakarzinom behandelt. Bei 52 Fällen lag ein DCIS vor. Bei diesen Patienten erfolgte eine SSM mit simultanem Brustaufbau durch Silikonimplantat. Eine zusätzliche strahlentherapeutische Behandlung wurde nicht durchgeführt. Die Nachbeobachtungszeit betrug im Median 65 Monate. Ergebnisse: Von den Patientinnen mit DCIS erlitten nur 2 ein lokoregionäres Rezidiv (3,8\%). Dies entspricht den Literaturangaben sowohl über Mastektomien als auch über SSM. 6 Patienten $(11 \%)$ erhielten bei Implantatproblemen einen Austausch durch freie Perforatorlappen (DIEP). Zusammenfassung: Die Ergebnisse bezüglich der Rezidivfreiheit sind bei der Mastektomie und der technisch schwierigeren SSM gleich. Die Lokalrezidivrate liegt in der Literatur bei diesen Techniken eindeutig günstiger als bei alleiniger lokaler Resektion. Bei Komplikationen oder subjektiven Beschwerden nach Alloplastik steht mit dem DIEP-Flap eine Alternative zur Verfügung. Diese Befunde rechtfertigen den Einsatz des Verfahrens beim DCIS als ästhetisch günstigere Alternative zur entstellenden Mastektomie.

\section{V24}

\section{Der freie Perforatorlappen vom Unterbauch (DIEP-Flap) Vergleich der Hebemorbidität von Perforatorlappen und TRAM-Flap}

*U. von Fritschen, C. Kässmann, G. Holle, K. Exner

Klinik für Plastische Chirurgie, Wiederherstellungs-und

Handchirurgie Markus-Krankenhaus, Frankfurt, Deutschland

Problemstellung: Die erhebliche Hebemorbidität des gestielt TRAMLappen ist in der Literatur gut beschrieben. Fettgewebsnekrosen werden bis zu $44 \%$ angegeben, partielle Lappennekrosen in 16\% und abdominale Hernien in bis zu 22\% der Fälle. In der mikrochirurgischen Technik als freier Lappen liegt die Komplikationsrate deutlich niedriger. Der vollständige Funktionserhalt der geraden Bauchwandmuskulatur beim 
DIEP-Lappen erhöht die Komplexität und die chirurgischen Anforderungen des Eingriffes erheblich. Die Frage, ob der technisch schwierigere Eingriff günstigere Ergebnisse erzielt bleibt bis heute offen. Patienten und Methode: Von 1996-2003 wurden bei 234 Patientinnen 291 DIEP-Lappen durchgeführt und die Verläufe prospektiv erfasst. Zusätzlich wurde be 39 konsekutiven Fällen präoperativ, bei Entlassung aus der stationären Behandlung, nach 2 Monaten und nach 2 Jahren eine funktionelle myosonografische Untersuchung durchgeführt. Ergebnisse: Partielle Lappennekrosen traten in 7 Fällen $(2,1 \%)$, partielle Fettgewebsnekrosen (alle Indurationen) vierzig mal auf $(13,7 \%)$, Nekrosen über $4 \mathrm{~cm}$ Durchmesser in $5,4 \%$ und ein vollständiger Lappenverlust in 6 Fällen $(2,1 \%)$. Eine funktionell relevante Bauchwandhernie konnte in 3 Fällen gefunden werden $(1,1 \%)$. Die myosonografische Untersuchung konnte nachweisen, dass in allen Fällen eine funktionsfähige Muskulatur vorliegt, die sich im postoperativen Verlauf identisch zur Gegenseite entwickelt. Die abschließende Untersuchung zeigt ein dem präoperativen Ausgangsbefund entsprechendes Resultat. Zusammenfassung: Die Präparation der PerforatorLappen ist eine anspruchsvolle Operation mit deutlicher Lernkurve. Be guter mikrochirurgischer Technik ist die Hebedefektmorbidität minimal Das ästhetische Resultat der Rekonstruktion ist durch die höhere Flexibilität bei der Lappenkonturierung den gestielten Techniken überlegen Der gestielte TRAM ist unter diesen Aspekten als historische Operationsmethode anzusehen.

\section{Freie Vorträge: Langzeitbeobachtung/Lebensqualität}

\section{V25}

\section{Einfluss einer Brustkrebserkrankung oder gynäkologisch- onkologischen Erkrankung auf die ökonomische und die partnerschaftliche Situation - eine Erhebung an 1030 Frauen}

${ }^{*}$ Peter A. Fasching ${ }^{1}$, Karen Nicolaisen-Murmann ${ }^{1}$, Michael P. Lux ${ }^{1}$, Christian Löhberg ${ }^{1}$, Mayada Bani' ${ }^{1}$, Hans G. Bender ${ }^{2}$,

Matthias W. Beckmann ${ }^{1}$

${ }^{1}$ Unifrauenklinik Erlangen, Erlangen, Deutschland

${ }^{2}$ Unifrauenklinik Düsseldorf, Düsseldorf, Deutschland

Einleitung: Die Auseinandersetzung mit einer Krebserkrankung beinhaltet soziale und ökonomische Herausforderungen: Diagnose, Operation, medikamentöse Behandlung, Bestrahlung, die Situation eines Rückfalls und deren Behandlung. Diese Ereignisse nehmen Einfluss sowohl auf die soziale als auch die finanzielle Situation der Patientinnen. In einem Kollektiv von 1030 Frauen mit Mammakarzinom (MaCa) oder gynäkologischen Tumoren (gynCa) wurde die Assoziation verschiedener sozialer partnerschaftlicher und finanzieller Aspekte mit Parametern der Erkrankung ermittelt. Material und Methoden: Von Februar 2000 bis Oktober 2002 wurden 1030 Frauen mit MaCa oder gynCa mittels eines standardisierten Fragebogens, welcher Parameter der Krankengeschichte, der Lebensqualität, der finanziellen, beruflichen und partnerschaftlichen Situation beinhaltete, befragt. Die verschiedenen Aspekte wurden im Hinblick auf ihre Assoziation mittels uni- und multivariater Analyse unter sucht. Ergebnisse: Die Patientinnen waren hauptsächlich unzufrieden mi ihrer Situation in den Bereichen Gesundheit (48,4\%), Sexualleben $(15,1 \%)$, Beruf $(13,9 \%)$ und Freizeit $(13,3 \%)$. Metastasen oder Rezidiv waren eindeutig mit beruflicher Unzufriedenheit $(\mathrm{OR}=2,05)$, einem Partnerverlust $(\mathrm{OR}=1,95)$ und sexueller Unzufriedenheit $(\mathrm{OR}=3,03)$ assoziiert. Zusammenfassung: Schul- und Berufsausbildung stehen in keinem Zusammenhang mit sozialer oder ökonomischer Zufriedenheit. Durch die Tumorerkrankung werden die Frauen am meisten in den Bereichen Gesundheit, Freizeit, Sexualleben und Beruf beeinträchtigt. Eine fortgeschrittene Erkrankung beeinträchtigt Partnerschaft und Sexualleben negativ. Im Rahmen der Sozialarbeit und psychoonkologischen Betreuung sollten ökonomischen und partnerschaftlichen Problemen besondere Beachtung geschenkt werden.

\section{V26}

\section{Entstehung von Angiosarkomen nach Brusterhaltender} Therapie des Mammakarzinoms - Initiierung eines Registers

\section{Jörg Thomas Hartmann, *Peter Hohenberger}

Chirurg. Univ. Klinik Mannheim, Sektion Chirurgische Onkologie und Thoraxchirurgie, Mannheim, Deutschland

Medizinische Klinik II, Universitätsklinikum, Tübingen, Deutschland

Problem: In der Nachsorge nach Mammakarzinom, insbesondere nach adjuvanter Bestrahlung, werden immer wieder Angiosarkome nach einer Latenzzeit von 5-10 Jahren beschrieben, bisher meist als Kasuistiken. Eine Inzidenz kann in der BR nicht beziffert werden. Aufgrund der ungesicherten Datenlage wurde der Aufbau eines nationalen Registers zur Erfassung der im deutschsprachigen Raum diagnostizierten Fälle initiiert. Das Register soll mögliche klinische und molekularbiologische Risikofaktoren für das Auftreten von Angiosarkomen bei Mammakarzinomen analysieren. Ziele der Studie:

Abschätzung der Inzidenz in der BR

Disposition von Patientinnen?

Interaktion mit Chemotherapie?

Interaktion mit Strahlenart/-feld/-dosis?

Vergleich primärer und sekundärer Angiosarkome bzgl. molekularbiologischer Charakteristika sowie klinisch (Behandlungsmodalitäten, Sequenz, Re-Bestrahlung)

Durchführung: Die Erfassung von Patienten mit Angio-sarkomen erfolgt auf einem einfach gestalteten Fragebogen, der bei der Studienleitung angefordert oder von den websites downgeloaded werden kann. Allen gröBeren Zentren, die Patienten behandeln, werden Teilnahmebögen aktiv zugeschickt. Auf den Prüfbögen werden alle Patientencharakteristika, sowie die gewählten Therapiestrategien mit minimalem Arbeitsaufwand dokumentiert. Patienten sollen retrospektiv ab dem Jahr 2000 und prospektiv eingeschlossen werden (geplant bis Ende 2007).

\section{V27}

\section{Ein "Case-By-Case-Management» Gynäko-Onkologischer Therapien zur Reduktion von Medikamentenkosten um 87,6\% innerhalb von 16 Monaten}

\section{*Volker R. Jacobs ${ }^{1}$, Jutta Thödtmann², Bettina Brunner ${ }^{1}$,} Marion Kiechle ${ }^{1}$

${ }^{1}$ Frauenklinik, Technische Universität, München, Deutschland; ${ }^{2}$ Apotheke, Technische Universität, München, Deutschland

Zielsetzung: State-of-the-Art onkologische Therapien sind mit bis zu $2000 €$ pro Zyklustag sehr teuer. Die Einnahmen unserer Frauenklinik für teilstationäre CTX betrugen 2002 nur pauschal $653 €$ bzw. $419 €$ für die stationäre Durchführung eines Zyklustages. Das Klinikbudget für Chemotherapien war gedeckelt; die erlaubten Fallzahlen ( $\mathrm{n}=943 / \mathrm{Jahr}$ ) wurden mit 1800 massiv überschritten. Ein Verlust von $340000 €$ war die Folge; die Verwaltung drohte mit der Streichung von Arztstellen. Wir stellen unser erfolgreiches Konzept zur Kostenreduktion vor. Materialien und Methoden: Nach Analyse der finanziellen Ist-Situation und aller beitragenden Faktoren wurde eine Vorkalkulation der CTX-Kosten für jede einzelne CTX durchgeführt, ein «Case-by-Case-Management» für die Zuordnung jeder einzelnen CTX zu einem kostendeckenden Abrechnungsmodus entwickelt und in einer Nachkalkulation alle verbliebenen Fehler eliminiert. Zielsetzung war, dass alle CTX kostendeckend sein müssen. Ergebnisse:Dieses «Case-by-Case-Management» führte zu Kosteneinsparungen bei Chemotherapeutika von absolut $523208 €$ in $2003(-58,7 \%)$, sowie weiteren $85894 €(-70,0 \%)$ von Januar bis April 2004, so dass die Schulden des Jahres 2002 wieder ausgeglichen und die angedrohte Stellenreduktion im ärztlichen Bereich zurückgenommen wurde. Dabei wurde weder das therapeutische Niveau abgesenkt, noch die Anzahl der durchgeführten Therapien wesentlich verringert, obwohl die Fallzahlen auch im Jahr 2003 wieder um 71,8\% über den erlaubten Fallzahlen lagen. Zusammenfassung: Onkologische Therapien können in einer Universitätsklinik auf hohem therapeutischen Niveau mit dem «Case-by-CaseManagement» nicht nur kostendeckend, sondern gewinnbringend erbracht werden, so dass mehrere neue Mitarbeiter eingestellt werden können. Ein stringentes Kostenmanagement eines engagierten Klinikers mit Weisungskompetenz und mit täglicher Kontrolle der teilstationären Chemoeinheit und aller Abrechnungen ist dafür die Voraussetzung. 
V28

\section{Zertifizierung von 9 Brustzentren aus Sicht eines FAB- Auditors (OnkoZert)}

\section{Gernot Maiwald}

Klinik für Brusterkrankungen, Behandlungszentrum, Vogtareuth, Deutschland

Der Weg des deutschlandweiten Zertifizierungsprogramms für interdisziplinäre Brustzentren führte auf der Basis des Fachanforderungskatalogs für Brustzentren (FAB) zur Erteilung des Zertifikats in bislang 23 Brustzentren.

Unter den Zentren befanden sich 7 universitäre und 16 städtische Einrichtungen. Aus Sicht des FAB-Fachexperten fanden sich in den durch ihn selbst auditierten 9 Einrichtungen wiederholt Schwachpunkte, die zu einem umfangreichen Maßnahmenkatalog im 1. Jahresaudit führen.

In der Regel besteht nach dem Erstaudit Dokumentationsbedarf in der Ausweisung von Kennzahlen des FAB. Ferner fehlen klinikspezifische Langzeitstatistiken für Überlebensraten. Die im Erhebungsbogen aufgeführten Leistungskennzahlen (z.B. Anzahl der primären Mammakarzinome, Verhältnis BET/Ablatio, Anzahl pTis bei Ersteingriff, entdeckte Karzinome bei offener Biopsie maligne/benigne, Anzahl Patienten mit Nachresektionen, Infektionsstatistik, Kompli-kationsstatistik, Anzahl der Untersuchungen in den Funktionsbereichen) müssen im Zertifizierungsaudit in ihrem Zustandekommen nachvollzogen werden kön-nen. Probleme gibt es auch bei der Ausweisung der Fortbildung des Assistenzpersonals, Qualitätszirkeln, Patienten- und Mitarbeiterzufriedenheitsanalysen, Lebensqualitätsstatistiken und der interdisziplinären Vernetzung zwischen Niedergelassenen und Klinik.

Zusammenfassung: Der von der DKG/DGS zu Grunde gelegte FAB-Katalog stellt für viele Häuser eine schwer erfüllbare Zielvorgabe dar. Eine kostenintensive, Berater-gestützte Vorbereitung und eine aufwendige klinikspezifische Do-kumentation mit infrastruktureller Umstrukturierung sind Voraussetzung zur Erlangung des Zertifikats nach ISO:9001 und der DKG/DGS.

\section{V29}

Patientenzufriedenheit nach autologem Brustwiederaufbau mit Diep-/S-Gap-/Tram- Flap am Behandlungszentrum Vogtareuth

*Svenja Preßler, Gernot Maiwald, Alberto Peek

Klinik für Brusterkrankungen, Abteilung Plastische Chirurgie,

Behandlungszentrum, Vogtareuth, Deutschland

Die Nachfrage nach einer Brustrekonstruktion mit autologem Gewebe nach einer Ablatio mammae hat in den letzten Jahren deutlich zugenommen, da eine Rekonstruktion mit alloplastischen Materialien langfristig oft $\mathrm{zu}$ unbefriedigenden kosmetischen Ergebnissen führt.Wir führten von 1997 bis 2004303 autologe Brustrekonstruktionen durch (174 uni-, 13 bilaterale DIEP-Flaps, 54 uni-, 1 bilateralen S-Gap-Flap, 59 uni-, 2 bilaterale freie TRAM-Flaps). Im Rahmen einer Patientenbefragung sollte nun die Zufriedenheit in Hinblick auf das Langzeitergebnis, die Lebensqualität und das Selbstwertgefühl untersucht werden. Material und Methoden: An 276 Patientinnen wurde ein Fragebogen versandt.Er umfaßt 11 Fragen, die mit einer Note von 1 bis 6 beantworte werden sollten.Er beinhaltet Fragen zur Lebensqualität,zum Selbstwertgefühl, der Akzeptanz des Ergebnisses durch die Patientin selbst und durch ihre Umwelt. Ergebnisse: Bis zum jetzigen Zeitpunkt lagen uns 116 Rückläufer vor. Nach Auszählung zeigt sich eine eindeutige Tendenz, ca. _ der Patientinnen sind mit dem Operationsergebnis zufrieden bis sehr zufrieden. Es zeigt sich kein deutlicher Unterschied zwischen den unterschiedlichen Verfahren der Brustrekonstruktion. Diskussion: Die Brustrekonstruktion mit autologem Gewebe vom Unterbauch (DIEP-Flap, TRAM-Flap) oder vom Gesäß (S-Gap-Flap) nach Ablatio mammae ist eine Methode, die überwiegend zu guten kosmetischen Ergebnissen und zu einer hohen Patientenzufriedenheit führt. In einem Zeitraum von 7 Jahren lag die Lappenverlustrate in unserem Krankengut bei unter $3 \%$. Aufgrund der geringeren Hebedefektmorbidität des DIEP-Flaps, der leichteren Formbarkeit im Vergleich zum TRAM-Flap sowie der konstanteren Gefäßversorgung im Vergleich zum S-Gap-Flap stellt diese Operationsmethode in unserer Klinik den führenden Eingriff zur autologen Brustrekonstruktion mit guten Ergebnissen dar.Für den gestielten TRAM-Flap sehen wir heute aufgrund des Hebedefektes keine Indikation mehr.

\section{V30 \\ Leitliniengestützte Qualitätsdarstellung für die Mammachirurgie - Ergebnisse aus dem BOS-Verfahren}

*Anne Reiter, Klaus Döbler, Volker D. Mohr Bundesgeschäftsstelle Qualitätssicherung gGmbH, Düsseldorf, Deutschland

Zielsetzung: Ziel der vergleichenden Qualitätssicherung ist die Verbesserung der Ergebnisqualität in der stationären Versorgung durch Benchmarking. Materialien und Methoden: Zum fairen Vergleich der Behandlungsqualität zwischen den Krankenhäusern in Deutschland sind bei der BQS für den Bereich Mammachirurgie 18 Qualitätsindikatoren implementiert. Die Entwicklung und Evidenzbewertung dieser Indikatoren erfolgte durch eine interdisziplinär zusammengesetzte Fachgruppe auf der Basis methodisch hochwertiger Leitlinien, wissenschaftlicher Gutachten und internationaler Studien. Ebenfalls leitliniengestützt konnten Referenzbereiche für Krankenhäuser für fast alle Indikatoren festgelegt werden. Unterschiedliche Patientinnenkollektive (Case mix) können zu Verzerrungen in den durch Qualitätsindikatoren gemessenen Behandlungsergebnissen führen und werden deshalb mittels statistischer Methoden (Adjustierung) berücksichtigt. Für Krankenhäuser mit sehr kleinen Fallzahlen sind die Ergebnisvergleiche statistisch nicht abgesichert und müssen deshalb separat dargestellt und interpretiert werden. Bei Krankenhäusern mit Auffälligkeiten in der Versorgungsqualität werden Ursachen dafür im Dialog auf Landesebene erörtert. Die so gewonnenen Informationen können genutzt werden für die kontinuierliche Qualitätsverbesserung im Sinne des internen Qualitätsmanagements und einen externen Vergleich der Krankenhausergebnisse. Ergebnisse: Anhand ausgewählter Qualitätsindikatoren, beispielsweise «Indikation zur brusterhaltenden Therapie» wird das Konzept der vergleichenden Qualitätssicherung und die aktuellsten Daten der Versorgungssituation in Deutschland dargestellt. Zusammenfassung: Zur externen Qualitätsdarstellung wurden bei der BQS in der Mammachirurgie 18 Qualitätsindikatoren entwickelt auf der Basis evidenzbasierter Leitlinien. Die krankenhausbezogenen Ergebnisse geben dabei wichtige Impulse für das interne Qualitätsmanagement und einen externen Vergleich der Krankenhausergebnisse. So kann die Versorgungsqualität in Deutschland transparent dargestellt und mittelfristig die Ergebnisqualität verbessert werden. 
V31

Brusterhaltende Therapie von Mammakarzinomen mit niedrigem Rezidivrisiko: Stellenwert von Strahlentherapie und Tamoxifen nach Tumorektomie. Erste Ergebnisse der GBSG 5-Studie

${ }^{*}$ R. Sauer ${ }^{1}$, K. -J. Winzer ${ }^{2}$, W. Sauerbrei ${ }^{3}$, E. Schneller ${ }^{4}$, W. Jaeger ${ }^{5}$ M. Braun ${ }^{6}$, J. Dunst ${ }^{7}$, O. J. Ott ${ }^{1}$, T. Liersch ${ }^{8}$, M. Zedelius ${ }^{9}$, K. Brunnert ${ }^{10}$, W. Guski1 ${ }^{11}$ C. Schmoor ${ }^{3}$, M. Schumacher ${ }^{3}$

${ }^{1}$ Department of Radiotherapy, Universitätsklinikum, Erlangen, Deutschland

${ }^{2}$ Department of Surgery, Universitätsklinikum Charité, Berlin, Deutschland

${ }^{3}$ Institute of Medical Biometry and Medical Informatics,

Universitätsklinikum, Freiburg, Deutschland

${ }^{4}$ Department of Gynaecology, Allgemeines Krankenhaus, Hagen,

Deutschland

${ }^{5}$ Department of Gynaecology, Universitätsklinikum, Erlangen,

Deutschland

${ }^{6}$ Department of Gynaecology, Zentralkrankenhaus, Bremen-Nord

Deutschland

${ }^{7}$ Department of Radiotherapy, Universitätsklinikum, Halle,

Deutschland

${ }^{8}$ Department of Surgery, Universitätsklinikum, Göttingen,

Deutschland

${ }^{9}$ Department of Gynaecology, Diakonissenkrankenhaus, Karlsruhe, Deutschland

${ }^{10}$ Department of Gynaecology, Klinikum, Osnabrück, Deutschland

${ }^{11}$ Institute of Pathology, Universitätsklinikum Charité, Berlin,

Deutschland

Ziele: Zur Frage, ob auch bei Mammakarzinompatientinnen mit geringem Rezidivrisiko nach einer Tumorektomie im Gesunden eine postoperative Ganzbrustbestrahlung notwendig ist, wurde eine randomisierte Studie mit vier Armen durchgeführt (Arm 1: keine adjuvante Therapie vs Arm 2: Strahlentherapie vs. Arm 3: Antihormontherapie mit Tamoxifen vs. Arm 4: Strahlentherapie und Antihormontherapie mit Tamoxifen). Patientinnen und Methoden: Zwischen 1991 und 1998 wurden 347 auswertbare Patientinnen rekrutiert. Als low-Risk galten invasive Mammakarzinome bis maximal $20 \mathrm{~mm}$ Durchmesser mit histologisch verifiziertem tumorfreien Randsaum, ohne axillären Lymphknotenbefall, ohne EIC, mit G1/2-Differenzierung, positivem Hormonrezeptorstatus, ohne Fernmetastasen (M0) und ein Patientinnenalter von 45-75 Jahren. Die Strahlentherapie in den Armen 2 und 4 sah eine Ganzbrustbestrahlung mit 25x 2 Gy bis 50 Gy sowie einen kleinvolumigen Elektronen-Boost mit 5-6x 2,0 Gy bis zu einer Gesamtreferenzdosis von 60-62 Gy vor. Als Antihormontherapie wurde in den Armen 3/4 Tamoxifen $30 \mathrm{mg} / \mathrm{Tag}$ für eine Dauer von zwei Jahren verabreicht. Die mediane Nachbeobachtungszeit betrug 5,9 Jahre. Ergebnis: Die Rate an invasiven ipsilateralen Rezidiven betrug im Beobachtungsarm (Arm 1) 28\% und in den Behandlungsarmen (Arm 2,3 und 4) jeweils 3\%. Das relative Risiko (RR), ein nochmaliges Krankheitsereignis zu erleiden, wurde im Vergleich zum Beobachtungsarm $(\mathrm{RR}=1)$ durch eine adjuvante Therapie deutlich reduziert (Arm 2: RR =0,35; Arm 3: $\mathrm{RR}=0,28$; Arm 4: RR =0,39. Der Unterschied war statistisch hochsignifikant ( $p=0,0001)$. Schlussfolgerungen: Auch bei Patientinnen mi einem geringen Lokalrezidivrisiko ist der Verzicht auf Strahlentherapie und gegebenenfalls Antihormontherapie nicht gerechtfertigt. Bei der kurzen Nachbeobachtungszeit ist allerdings noch keine Aussage hinsichtlich der langfristigen Auswirkungen auf das Gesamtüberleben möglich.

V32

Patientinnenseminare - ein psychosoziales Angebot zur verbesserten Nachsorge brustkrebsbetroffener Frauen

*Jürgen Schwickerath ${ }^{1}$, Elmar Reuter ${ }^{2}$, Barbara Rehse ${ }^{2}$

${ }^{1}$ Frauenklinik St. Martinus-Hospital, Olpe, Deutschland,

${ }^{2}$ Psychoonkologische Schwerpunktpraxis, Olpe, Deutschland

Zielsetzung: Die Diagnose Krebs stellt für die meisten der betroffenen Frauen einen schweren Schock dar,der sie häufig in eine existenzielle Lebenskrise führt.Um sie in der Bewältigung ihrer Erkrankung zu unterstützen,wird von den Frauen der Wunsch nach einer psychosozialen/psycho- onkologischen Hilfe geäußert. Aus der seit 1998 bestehenden Kooperation zwischen der Frauenklinik und einer Psychotherapiepraxis (Schwerpunkt Psychoonkologie)entstand 2001 der Gedanke, so genannte Patientenseminare für betroffene Frauen einzurichten. Material/Methode: Ziel der(bisherigen 4) Seminare ist es,d ie Kompetenz der Patientinnen zu stärken. Sie sollen an den anstehenden Entscheidungen besser teilnehmen können,sich unterstützt fühlen und ein allgemeines Gesundheitswissen aufbauen.Ihre medizinischen Befunde,ihre krankheitsbezogenen und soziographischen Daten werden am Anfang und am Ende der Veranstaltungsreihe nach zirka 6 Monaten dokumentiert (HADS, BSI, SOC-9L, FSozu, EORTC). Gleichzeitig kommt ein Fragebogen mit Fragen zur Akzeptanz des Seminars, zur Einschätzung des Informationszuwachses und eine Zufriedenheitseinschätzung zum Einsatz. Ergebnisse: Bei den meisten Teilnehmerinnen steigen die Erwartungen bzgl.der Wirksamkeit von Behandlungen an, wobei offensichtlich der Nutzen aller Optionen - Medizin, Psychologie, Naturheilkunde, körperorientierte Verfahren - gleichmäßig positiv bewertet wurde. Der Glaube, dass verbesserte Selbstfürsorge den Krankheitsverlauf günstig beeinflussen kann, zeigt den stärksten Anstieg. Zusammenfassung: Die Ergebnisse sprechen für ein gesteigertes Lebensgefühl und für eine bessere Lebensqualität mit einem gleichzeitigen hohen Maß an Selbstfürsorge. Die «Patientinnenkompetenz» wurde nach Abschluss der Seminare als deutlich verbessert und positiv eingestuft. Mut und Selbstbewusstsein, mit ihren Behandlern auf «gleicher Augenhöhe» sprechen zu können, sei über die Vermittlung von Wissen und Sachverstand gefördert worden. Die Ergebnisse zeigen, dass ein solches Angebot für einen Teil der betroffenen Frauen unverzichtbar ist.

V33

\section{Veränderungen des Körperbildes bei Mammakarzinom-} patientinnen in der frühpostoperativen Phase

*Friederike Siedentopf, Martina Nagel, Heribert Kentenich Frauenklinik, DRK-Kliniken Westend, Berlin, Deutschland

Zielsetzung: Wie unterscheidet sich die Körperwahrnehmung von Mammakarzinompatientinnen früh postoperativ von der eines gesunden Kontrollkollektivs? Besteht eine Abhängigkeit vom operativen Vorgehen? Material und Methoden: Postoperative Befragung von 60 Patientinnen nach Erstoperation eines Mammakarzinoms mit dem Fragebogen zur Beurteilung des eigenen Körpers (FBeK). Vergleich mit einer Kontrollgruppe (40 gesunde Frauen zur jährlichen Krebsfrüherkennungsuntersuchung in einer gynäkologischen Praxis). Ergebnisse: Die Mammakarzinompatientinnen erleben sich als attraktiver und berichten gleichzeitig über mehr Selbstvertrauen als die gesunde Kontrollgruppe.Die postoperative Zufriedenheit in der Gruppe der mastektomierten Patientinnnen (mit und ohne Sofortrekonstruktion) ist höher als bei brusterhaltend operierten Frauen. Frauen, die eine Sofortrekontruktion erhielten, legten besonderen Wert auf Mitbestimmung bei der Wahl der OP-Methode. Zusammenfassung: Ein höheres Mass an Mitbestimmung erweist sich als vorteilhaft für die postoperative Zufriedenheit.Die frühpostoperative Reaktion auf die Diagnose eines Mammakarzinoms ist durch Abwehrmechanismen geprägt. Die zu erwartende Verunsicherung des Körperselbstbildes scheint erst zu einem späteren Zeitpunkt einzutreten. Umso wichtiger ist das Auffangen der Patientin in einer nicht nur medizinisch, sondern auch psychosozial und psychoonkologisch orientierten Nachsorge. 
V34

Zoledronsäure ist eine sehr effektive und sichere Behandlung der Knochenschmerzen bei ossär metastasierten Karzinomen - Ergebnisse einer prospektiv, randomizierten Studie

${ }^{*}$ E. Solomayer ${ }^{1}$, A. Kretzschmar ${ }^{2}$, T. Wiegel ${ }^{3}$, S. E. Al-Batran ${ }^{4}$, H. F. Hinrichs ${ }^{5}$, M. Kindler ${ }^{6}$, T. Steck ${ }^{7}$, H. J. Illiger ${ }^{8}$, U. Haus ${ }^{9}$,

A. Kirner ${ }^{9}$, G. Ehninger ${ }^{10}$

${ }^{1}$ Universitätsklinikum, Tübingen, Deutschland

${ }^{2}$ Robert-Rössle-Klinik, Berlin, Deutschland

3Universitätsklinikum Benjamin Franklin, Berlin, Deutschland

${ }^{4}$ Krankenhaus Nordwest, Frankfurt, Deutschland

${ }^{5}$ Oldenburg, Deutschland

${ }^{6}$ Berlin

${ }^{7}$ Klinikum Chemnitz, Chemnitz, Deutschland

${ }^{8}$ Klinikum Oldenburg, Oldenburg, Deutschland

${ }^{9}$ Novartis Pharma GmbH, Nürnberg, Deutschland

10Universitätsklinikum TU, Dresden, Deutschland

Zielsetzung: Knochenmetastasen solider Tumoren führen zu gravierenden Komplikationen wie Knochenschmerzen, pathologische Frakturen und Hyperkalzämie. Das Bisphosphonat Zoledronat hemmt die Funktion der Osteoklasten und führt dadurch zu einer Verbesserung der skelettalen Komplikationen. Materialien und Methoden: Die Effektivität des Bisphosphonats Zoledronat wurde bei Patienten mit ossären Metastasen untersucht. Bisphosphonat-naive Patienten mit mindestens einer nachweisbaren Knochenläsion wurden in diese prospektive Multicenter-Studie rekrutiert. Die Knochenmetastasen stammten von unterschiedlichen Primärtumoren. Alle Patienten erhielten maximal 12 Infusionen von $4 \mathrm{mg}$ Zoledronat alle 3-4 Wochen. Primäre Studienziele waren die Änderungen der Schmerzwahrnehmung (100 mm visual analog scale für Knochenschmerzen -VAS), und Analgetikaverbrauch (gemessen auf einer fünfPunkte Skala von 0 bis 4 gemäß analgetischer Wirksamkeit). Die Dokumentation erfolgte durch Patiententagebuch. Wir präsentieren die Auswertung nach 24 Wochen. Ergebnisse: Es wurden 606 Patienten aus 67 Zentren rekrutiert. 408 Patienten wurden im Verlauf suffizient dokumentiert und waren für die Effektivitätsanalyse auswertbar. Das Alter der Patienten (61\% Frauen) variierte von 31 bis 90 Jahre. Die meisten Primärkarzinome waren Mamma- (49\%) und Prostatakarzinom (18\%). 319 Patienten hatten ihre Behandlung bis zu 24 Wochen. Der mittlere VAS-Wert für Schmerzwahrnehmung nahm um 13,8+/-30,6 von 36,9+/-28,2 auf $23,1+/-23,8$ ab $\mathrm{p}<0,0001$, t-test. Der mittlere Analgetische Score nahm um 0,37+/-1,30 von 1,65+/-1,56 auf 1,28+/-1,61)ab (p<0,0001, t-test). Zusammenfassung: Zoledronat ist bei der Therapie der Knochenmetastasen zahlreicher Primärkarzinome sehr effektiv. Dieses hochpotente Bisphosphonat reduziert die Knochenschmerzen und erhöht die Lebensqualität.

\section{V35 \\ Epidemiologie des Mammakarzinoms bei Frauen unter 35 Jahren}

\section{R. Stabenow}

Gemeinsames Krebsregister der Länder Berlin, Brandenburg, Mecklenburg-Vorpommern, Sachsen-Anhalt und der Freistaaten Sachsen und Thüringen (GKR), Deutschland

Material und Methoden: Bevölkerungsbezogene Analysen von Mammakarzinomen im Alter unter 35 Jahren in Deutschland sind sehr selten. Das GKR ist mit einer Basispopulation von 17 Millionen das größte epidemiologische Krebsregister in Deutschland und verfügt über Daten beginnend mit dem Diagnosejahr 1961. Wir analysierten die Daten der Diagnosejahre 1999-2001. Es wurden Inzidenzraten, Stadienverteilungen, Sublokalisationen und histologische Tumortypen ausgewertet. Ergebnisse: Im Zeitraum 1999-2001 wurden 433 invasive Mammakarzinome (ICD-10 C50) gemeldet. Das entspricht einem Anteil von 15\% an allen Krebsneuerkrankungen, die im Alter unter 35 Jahren auftreten. Damit ist nur der Gebärmutterhalskrebs in dem Alter häufiger (16\%). Die rohe Inzidenzrate beträgt 4,4/100 000 und die altersstandardisierten Raten 3,4/100 000 (ESR) bzw. 2,6/100 000 (WSR). Die altersstandardisierte Rate verdoppelte sich von Anfang der 60er bis Ende der 70er Jahre und blieb seitdem nahezu konstant. Die T-Stadien aus TNM verteilen sich wie folgt: T0 5\%, T1a+1b 10\%, T1c+T1x 38\%, T2 37\%, T3 6\% und T4 4\%. Die Sta- dienverteilung ist etwas ungünstiger als bei Patienten mittleren Alters (35-69 Jahre), insbesondere gegenüber der Zielgruppe des Mammografiescreenings (50-69 Jahre), bei der der Anteil der Tumoren $<10$ mm (T0 bis T1b) $20 \%$ beträgt. Von den in den Quadranten lokalisierten Karzinomen entfallen 62\% auf den oberen äußeren Quadranten (oberer innerer Q. $18 \%$, unterer äußerer Q. 11\%, unterer innerer Q. 9\%). 77\% aller invasiven Tumoren sind duktale und $8 \%$ lobuläre Karzinome.

\section{V36 \\ Klinische Krebsregister als Instrumente der Qualitäts- sicherung in der Onkologie}

\section{Vergleich der Daten Tumorzentrum Land Brandenburg - Tumorzentrum München - Tumorzentrum Regensburg}

${ }^{*}$ Anett Tillack ${ }^{1}$, Monika Klinkhammer-Schalke ${ }^{2}$, Jutta Engel ${ }^{3}$ Knut Wenzelides ${ }^{1}$, Ferdinand Hofstädter ${ }^{2}$, Dieter Hölzel ${ }^{3}$ ${ }^{1}$ Tumorzentrum Land Brandenburg, Frankfurt(Oder), Deutschland ${ }^{2}$ Tumorzentrum Regensburg, Regensburg, Deutschland ${ }^{3}$ Tumorzentrum München, München, Deutschland

Fragestellung: Im Land Brandenburg und in Bayern arbeiten klinische Krebsregister und erfassen die Daten von Mammakarzinompatientinnen von der Diagnose über die Therapie bis hin zur Nachsorge. Ist mit einer solchen Datenbasis ein Qualitätsmanagement möglich? Materialien: In den genannten drei Tumorzentren werden seit mehr als zehn Jahren klinische Krebsregister geführt und Daten von Mammakarzinompatientinnen erfasst und in regelmäßigen Abständen nach festgelegten Qualitätsindikatoren ausgewertet. Ergebnisse: Es werden Inzidenz, Mortalität, Tumorstadien und Therapien beim Mammakarzinom im Einzugsgebiet der drei genannten Tumorzentren verglichen. Der Vergleich der Daten zeigt, dass es möglich ist die Qualität der Früherkennung in diesen Bundesländern zu beurteilen. Weiter wird belegt, dass relevante Qualitätsindikatoren zur Umsetzung der Leitlinien für die Therapie verfügbar sind. Mit diesen Daten wird in den genannten Tumorzentren im Rahmen von Projektgruppen Qualitätssicherung in der Onkologie seit Jahren aktiv betrieben und die Versorgung unterstützt. Damit kann in Regionen, in denen klinische Krebsregister effizient arbeiten, das DMP Brustkrebs unter Einbeziehung dieser Register durchgeführt werden.Der Dokumentationsaufwand für den meldenden Arzt wird minimiert und Parallelstrukturen werden vermieden. Schlussfolgerung: Mit den Daten klinischer Krebsregister kann, wie in diesen beiden Bundesländern seit Jahren praktiziert, die Qualität der Früherkennung, Diagnostik und Therapie beim Mammakarzinom und allen anderen Krebserkrankungen beurteilen werden. Mit diesen Daten sind die beim DMP Brustkrebs aufgeworfen Fragen bereits seit Jahren beantwortet.

\section{Freie Vorträge: Adjuvante Therapie}

\section{V37}

\section{Plastisch-chirurgische Therapie von Paravasat-Nekrosen an der oberen Extremität}

*Holger Bannasch, Stefan Krischak, Andreas Wenger, G. Björn Stark Abteilung Plastische und Handchirurgie,

Chirurgische Universitätsklinik Freiburg, Freiburg, Deutschland

Zielsetzung: Nekrosen nach Paravasation von Chemotherapeutika stellen eine erhebliche zusätzliche Belastung für die Patienten dar und erfordern eine interdisziplinäre Therapie unter Miteinbeziehung aller plastisch-chirurgischen Rekonstruktionsmöglichkeiten. Es kommt zu irreparablen, progredienten Gewebsschäden, welche ein frühes radikales Debridement und eine vaskularisierte Deckung erfordern. Methodik: In den Jahren 1998 bis 2004 wurden in der eigenen Abteilung 8 Patienten mit Nekrosen nach Paravasation (fast ausschließlich Anthracycline) im Bereich der oberen Extremität behandelt. Aufgrund der erheblichen Toxizität und Latenz bis zur definitiven Demarkation der Nekrosen erfolgten oft mehrere Operationen inklusive Debridement und Vakuumversiegelung sowie definitivem Wundschluss (Hautverpflanzung, lokale Lappenplastiken, freie Lappenplastiken). In den meisten Fällen war eine intensivierte spezielle Schmerztherapie erforderlich sowie eine begleitende psychologische $\mathrm{Be}$ - 
treuung der durch die Komplikation stark zusätzlich belasteten Patienten Zusätzliche rehabilitative Maßnahmen wurden so optimal wie möglich eingesetzt, waren durch die langwierige Behandlung aber mehrfach nur verzögert durchzuführen. Resultate: Bei allen Patienten erfolgten mehrere Operationen; unter Zuhilfenahme des gesamten plastisch-chirurgischen Arsenals konnte ein definitiver und kompletter Wundschluss erzielt werden. In allen Fällen verlängerte sich der stationäre Aufenthalt um mindestens zwei Wochen, die Behandlung der Grunderkrankung bzw. die Fortführung der Chemotherapie verzögerte sich ebenfalls bei allen $\mathrm{Pa}$ tienten. Schlussfolgerung: Paravasatnekrosen bei Chemotherapie werden in ihrem Ausmaß und dem resultierenden Dauerschaden häufig unterschätzt und stellen für Patienten in einer ohnehin kritischen Situation be maligner Grunderkrankung eine schwere Zusatzbelastung dar. Eine interdisziplinäre Therapie (Plastische Chirurgie, Onkologie, Psychologie, An ästhesie und rehabilitative Medizin) ist absolut erforderlich, aber auch bei Einsatz aller dieser Mittel ist oftmals keine völlige Wiederherstellung zu erzielen.

V38

\section{Intraoperative Radiotherapie (lort) als Boost bei der Strahlentherapie des Mammakarzinomes}

${ }^{*}$ L. Bauer ${ }^{1}$, U. Kraus-Tiefenbacher ${ }^{2}$, F. Wenz ${ }^{2}$, F. Melchert ${ }^{1}$

${ }^{1}$ Universitätsfrauenklinik, Mannheim, Deutschland

${ }^{2}$ Abteilung für Strahlentherapie, Universitätsklinikum, Mannheim, Deutschland

Zielsetzung: Die Restbrustbestrahlung ist das Standardverfahren bei Patientinnen mit Mammakarzinomen nach brusterhaltender Therapie (BET). Die meisten In-Brustrezidive treten in unmittelbarer Nachbarschaft zum Primärtumor auf. Daher wird das Tumorbett oft mit einer Boostbestrahlung versorgt, für die keine Standardtechnik existiert. Seit 2/2002 wird die IORT bei Patientinnen mit Mammakarzinomen in Mannheim mit einem mobilen Miniaturbeschleuniger (IntrabeamTM) durchgeführt. Methoden: Von 2/02 bis 10/03 wurden 99 Patientinnen nach BET und Axilladissektion intraoperativ bestrahlt. Bei 71 wurde die IORT als Boostbestrahlung des Tumorbettes mit 20 Gy durchgeführt. Das mediane Alter war 65 Jahre, die mediane Tumorgröße 13 mm (2-45). Die Behandlungszeit war im Median 20 Minuten (18-49). Nach abgeschlossene Wundheilung wurden die 71 Patientinnen mit einer homogenen Restbrustbestrahlung in üblicher Tangententechnik mit 46 Gy bestrahlt.Die IORT wurde von allen Patientinnen gut toleriert. 3 Patientinnen zeigten Wundheilungsstörungen, 2 ein Erythem ${ }^{\circ} \mathrm{II}$, alle Hautveränderungen heilten folgenlos ab. Nach einer Nachbeobachtungszeit von 26 Monaten zeigen die Patientinnen ein gutes kosmetisches Resultat. 1 Patientin mußte sich nach der BET aufgrund einer vorher nicht diagnostizierbaren Multifokalität einer sekundären Mastektomie unterziehen, 1 Patientin entwickelte 2 Monate nach der BET Halslymphknotenmetastasen. Eine weitere Patientin stellte sich 10 Monate nach der postoperativen Nachbestrahlung mit einem multifokalen Rezidiv mit Hautmetastasen in de behandelten Brust vor. Sie verstarb 4 Monate nach der sekundär durchgeführten Ablatio. Zusammenfassung: Die IORT ist eine komfortabe durchführbare Methode, um eine hohe Boostdosis im Tumorbett als Hauptrisikoareal zu applizieren. Durch die intraoperative Technik kann ein Verfehlen des eigentlichen Tumorbettes im bestrahlten Boostvolumen, wie häufig bei der perkutanen Strahlentherapie, vermieden werden
V39

Identifizierung von DNA-Methylierungsprofilen bei Tamoxifen behandelten, nodal- negativen Mammakarzinompatientinnen zur Risikoabschätzung

*Inga Bohlmann', Sabine Maier ${ }^{2}$, M. Schmitt ${ }^{4}$, Inko Nimmrich ${ }^{2}$, A. Marx², Volkmar Müller ${ }^{1}$, M. Kiechle ${ }^{4}$, Christoph Thomssen ${ }^{4}$ Fritz Jänicke ${ }^{1}$, Nadja Harbeck $^{4}$

${ }^{1}$ Universitätsfrauenklinik Eppendorf, Hamburg, Deutschland ${ }^{2}$ Epigenomics AG, Berlin, Deutschland

${ }^{3}$ Frauenklinik, Martin-Luther Universität, Halle, Deutschland

${ }^{4}$ Frauenklinik der Technischen Universität, München, Deutschland

Zielsetzung: Den heutigen Therapierichtlinien entsprechend erhalten viele Mammakarzinompatienten mit nodalnegativen und Hormonrezeptor -positiven Tumoren sowohl eine adjuvante Chemotherapie als auch eine endokrine Therapie. Ein Großteil der Patientinnen würde jedoch niemals ein Rezidiv erleiden und sind somit übertherapiert. Leider sind etablierte Risikofaktoren (Tumorgröße, Grading, Rezeptorstatus) nicht in der Lage, eine solche Übertherapie zu vermeiden. Das Ziel dieser Studie war es, DNA-Methylierungsmarker zu identifizieren, die mit einem niedrigen $\mathrm{Re}$ zidivrisiko nach Tamoxifenmonotherapie assoziiert sind. Material und Methoden: Primär wurde ein genomweites Screening für DNA-Methylierungsmarker durchgeführt, die mit einer erhöhten Rezidivrate nach Tamoxifentherapie assoziiert waren. Die vielversprechendsten Marker dieser Untersuchung wurden in einem unabhängigen Kollektiv $(\mathrm{n}=278)$ mittels Microarrayanalyse validiert. Ergebnisse: Es konnten mehrere DNA-Methylierungsmarker identifiziert werden, die signifikant mit dem rezidivfreien Intervall korreliert waren. (univariates Cox model, $\mathrm{p}<0,05)$. In der multivariaten Analyse lieferten die Methylierungsmarker zusätzliche Informationen, unabhängig von den bereits etablierten Faktoren wie Differenzierungsgrad, Tumorgröße und Östrogenrezeptorstatus. Nach der Kombination von drei Markern in einen sog. Methylierungs-Score (Median als Cut-off-level), konnte eine Subgruppe von 50\% der Patientinnen identifiziert werden, die nach alleiniger Tamoxifentherapie eine äußerst gute Prognose aufwiesen (Rezidivfreies Überleben 95\% nach 10 Jahren vs. $62 \%$ der übrigen Patientinnen). Eine Validierung der Ergebnisse an einem unabhängigen Patientenkollektiv erfolgt derzeit. Einer der Marker aus der Pilotstudie konnte bereits validiert werden $(\mathrm{p}<0,05)$. Zusammenfassung: Diese Ergebnisse zeigen deutlich, dass die Analyse spezifischer methylierter DNA-Bereiche zusätzliche Informationen über das Rezidivrisiko Tamoxifen behandelter Mammakarzinompatienten geben kann. Außerdem kann ein Niedrigrisikokollektiv nodal-negativer und hormonrezeptor-positiver Patientinnen identifiziert werden, denen vermutlich eine zusätzliche zytotoxische Behandlung erspart werden kann.

V40

Multiple Zytostatische Therapie bei Patientinnen mit

Metastasierten Mammakarzinom eine retrospektive Analyse von 104 Patientinnen

*Annette Dieing ${ }^{1}$, Peter Schmid ${ }^{1}$, Anne Röver ${ }^{1}$,

Carsten Oliver Schulz ${ }^{1}$, Galja Georgieva ${ }^{1}$, Nadja Ehlers $^{2}$, Silvia Lehenbauer-Dehm ${ }^{1}$, Bernd Flath ${ }^{1}$, Kurt Possinger ${ }^{1}$

${ }^{1}$ Medizinische Klinik m. S. Onkologie und Hämatologie,

Charité Campus Mitte, Berlin, Deutschland

${ }^{2}$ Frauenklinik der Universität UIm, UIm, Deutschland

Zielsetzung: Patientinnen mit metastasiertem Mammakarzinom, die nach der dritten Therapielinie einen Progress ihrer Erkrankung erleben und sich in einem gutem Allgemeinzustand befinden, erscheint eine weitere Therapie bei entsprechendem Wunsch vertretbar und wird häufig durchgeführt. Allerdings stehen zur Wirksamkeit der meisten eingesetzten Substanzen nach mehr als 3 Vortherapien kaum Daten zur Verfügung. Patienten und Methoden: In der vorliegenden retrospektiven Studie analysierten wir 104 Patientinnen mit metastasiertem Mammakarzinom, die drei oder mehr zytostatische Therapien erhielten. Ausgewertet wurden das Ansprechen und die Toxizität III und IV ${ }^{\circ}$. Ergebnisse: Das durchschnittliche Alter der Patientinnen bei ED des Mammakarzinoms war 49 Jahre, die durchschnittliche Zeit bis zur ED der Metastasierung betrug 40 Monate. 66 Patientinnen litten an einem Hormonrezeptor-positiven Tumor, Her2neu-Positivität lag in 31 Fällen vor. Die ausgewerteten Pa- 
tientinnen erhielten bis zu 10 zytostatische Therapien (5 Pt.). Dabei wurde in $44 \%$ aller Fälle eine Kombination von mindestens zwei Medikamenten eingesetzt. In einzelnen Fällen wurde objektives Ansprechen bis zur 7. Linie beobachtet, Clinical Benefit bis zur 10. Linie. Ansprechraten sind in Tabelle 1 dargestellt. Die Rate III- und IV-gradiger Toxizität lag zwischen $13 \%$ und $38 \%$. Dabei überwog die hämatologische Toxizität, die Rate höhergradiger nicht-hämatologischer Nebenwirkungen lag zwischen $0 \%$ und $14 \%$. Zusammenfassung: Zytostatische Therapien für das metastasierte Mammakarzinom sind nach intensiver Vorbehandlung mit vertretbarer Toxizität möglich und bei einem Teil der Patientinnen erfolgreich. Der Einfluss von Faktoren wie Ansprechen in früheren Linien, Art der Vorbehandlung und der eingesetzten Therapien sind an der kleinen Patientenzahl nur eingeschränkt zu beurteilen.

Tabelle 1. Ansprechraten

\begin{tabular}{|c|c|c|c|c|c|c|c|c|c|c|}
\hline Linie & 1. & 2. & 3. & 4. & 5. & 6. & 7. & 8. & 9. & 10 \\
\hline $\begin{array}{l}\mathrm{n} \\
\text { Objektives } \\
\text { Ansprechen } \\
\text { (CR/PR) }\end{array}$ & $\begin{array}{r}104 \\
31\end{array}$ & $\begin{array}{r}104 \\
28\end{array}$ & $\begin{array}{r}104 \\
14\end{array}$ & $\begin{array}{l}81 \\
11\end{array}$ & $\begin{array}{r}58 \\
2\end{array}$ & $\begin{array}{r}31 \\
1\end{array}$ & $\begin{array}{r}21 \\
1\end{array}$ & $\begin{array}{r}14 \\
0\end{array}$ & $\begin{array}{l}9 \\
0\end{array}$ & $\begin{array}{l}5 \\
0\end{array}$ \\
\hline $\begin{array}{l}\text { Clinical } \\
\text { Benefit } \\
\text { (CR/PR/SD } \\
\geq 6 \text { Monate) }\end{array}$ & 59 & 45 & 28 & 25 & 7 & 11 & 6 & 1 & 2 & 1 \\
\hline
\end{tabular}

\section{V41}

Einfluss einer standartisierter Chemotherapie mit Adriamycin und Cyclophosphamid (AC) auf Werte der DXA Messung sowie Werte der quantitativen Ultrasonometrie bei prämenopausalen Frauen mit primärem Mammakarzinom

*May Gottschalk, Peyman Hadji, Carolin Maskow, Volker Ziller, Eva Funk, Uwe Wagner

Klinik für Gynäkologie, gyn. Endokrinologie und Onkologie der Philipps - Universität, Marburg, Deutschland

Zielsetzung: Inhalt dieser Studie war den Einfluss einer standartisierten Chemotherapie mit 4 Zyklen Adriamycin und Cyclophosphamid (AC) auf Werte der DXA sowie Werte der quantitativen Ultrasonometrie (QUS) bei prämenopausalen Frauen mit primärem Mammakarzinom zu untersuchen. Material und Methoden: Insgesamt wurden 53 Patientinnen mit einem mittleren Alter von 47,5 \pm 9,4 Jahre mit neu diagnostiziertem primären Mammakarzinom und geplanter adjuvanten Chemotherapie $\mathrm{AC}$ in die Studie eingeschlossen. Zeitgleich wurden gesunde prämenop. Frauen untersucht die sich in Alter und BMI gegenüber der Mammakarzinom Pat. nicht unterschieden. Frauen mit Knochenmetastasen, Osteoporose, knochenstoffwechselbeeinflussenden Erkrankungen und/ oder Medikamenten wurden ausgeschlossen. Gemessen wurde die Knochendensitometrie per DXA (DPX-L, GE/ Lunar) an Wirbelsäule und Schenkelhals, die quantitative Ultrasonometrie mittels Achilles (GELunar) am Os Calcaneus und mittels Bone- Profiler (IGEA) an den Phlangen.Messzeitpunkt war vor Chemotherapiebeginn, nach 6 und nach 12 Monaten. Es erfolgte eine matched- pair Analyse zwischen Mammakarzinom- Patientinnen und Kontrollen. Ergebnisse: Die DXA- Messungen zeigte eine lineare signifikante Erniedrigung des t- und z scores in Wirbelsäule und Schenkelhals bei Patientinnen unter Chemotherapie $(\mathrm{p}<0,001)$. Die Werte der QUS zeigten im Bereich des Os Calcaneus ebenfalls diesen Verlauf des t- und z-scores, bei den Phalangen ergab sich der größte Unterschied zwischen baseline und 12 Monaten. Zusammenfassung: Das Ergebnis unserer Fall- Kontrollstudie war ein stark negativer Einfluss einer Chemotherapie mit AC auf die Knochendensitometriewerte bei prämenopausalen Frauen mit primärem Mammakarzinom. Dieser Effekt konnte sowohl mit der DXA- Messung wie auch mit der QUS gezeigt werden.Um den Mechanismus des Einflusses der Chemotherapie auf den Knochenstoffwechsel zu verdeutlichen wären Longitudinalstudien wünschenswert.
V42

Prädiktive Bedeutung von uPA/PAI-1 für Ansprechen auf adjuvante Chemotherapie beim Mammakarzinom durch Metaanalyse $(n=8,377)$ validiert

${ }^{*} N$ Harbeck ${ }^{1}$, RE Kates ${ }^{1}$, MP Look ${ }^{2}$, JA Foekens ${ }^{2}$, M Schmitt ${ }^{1}$, F Jänicke ${ }^{3}, C$ Thomssen ${ }^{4}$, für die Pooled Analysis Study der EORTC Receptor and Biomarker Group (RBG)

${ }^{1}$ Frauenklinik TU München, München, Deutschland

${ }^{2}$ ErasmusMC, Rotterdam, Niederlande

${ }^{3}$ Universitätsfrauenklinik-Eppendorf, Hamburg, Deutschland

${ }^{4}$ Martin Luther Universität, Halle, Deutschland

Zielsetzung: Die prognostische Bedeutung der Invasionsfaktoren uPA/PAI-1 beim primären Mammakarzinom wurde bereits durch eine prospektive multizentrische Therapiestudie und eine Metaanalyse auf höchstem Evidenzniveau validiert. Kürzlich zeigte eine große retrospektive Studie die prädiktive Bedeutung dieser Faktoren. Materialien und Methoden: Die prädiktive Bedeutung von uPA/PAI-1 hinsichtlich des Ansprechens auf adjuvante Therapie wurden mittels 18 Datensätzen ( $\mathrm{n}=8,377$ ) der Pooled Analysis Study der EORTC Receptor and Biomarker Group (RBG) validiert. Die mediane Nachbeobachtungszeit betrug 79 Monate mit unabhängig von uPA/PAI-1 getroffenen Therapieentscheidungen. uPA/PAI-1 wurden mittels ELISA in Primärtumorextrakten bestimmt, die Metaanalyse durch eine Rangtransformation der Meßergebnisse. Ergebnisse: Zeitvariierende Überlebensanalysen (stratifiziert nach Zentrum) zeigten, dass die adjuvante Chemotherapie mit einem erhöhten Benefit (HR 0,60, KI 0,41-0,87) bei Patientinnen mit hohem uPA/PAI-1 assoziiert war, v.a. um Rezidive innerhalb der ersten 3 Jahre zu verhindern, sowie bei Patientinnen mit 0-3 befallenen Lymphknoten (HR 0,51, KI 0,31-0,85). Die Cox-Analyse zeigte keine Interaktion zwischen adjuvanter endokriner Therapie (ET) und uPA/PAI-1. Es wurde jedoch eine Niedrigrisikogruppe (nodal-negativ, rezeptorpositiv, uPA/PAI-1 niedrig) mit exzellentem 10 Jahres Überleben von 92\% unter adjuvanter ET identifiziert. Zusammenfassung: Die signifikante unabhängige prädiktive Bedeutung von uPA/PAI-1 für das Ansprechen auf adjuvante Chemotherapie beim primären Mammakarzinom konnte durch diese Metaanalyse bestätigt werden, ohne Evidenz für eine anhand von uPA/PAI-1 definierte Subgruppe, die nicht von einer ET profitieren würde. Unsere Daten deuten daraufhin, dass alle Patientinnen mit hohem uPA/PAI-1 -unabhängig von ihrem Nodalstatus- eine adjuvante Chemotherapie erhalten sollten. Weiterhin kann der uPA/PAI-1-Status bei Indikation zwischen einer rein endokrinen oder einer chemo-endokrinen Therapie zur Therapieentscheidung beitragen.

\section{$\mathrm{V} 43$}

\section{COX-2-Überexpression in Mammakarzinomen-}

\section{Assoziation mit HER2-Amplifikation und p53-Mutation und} schlechter Prognose

${ }^{*}$ Arndt Hartmann ${ }^{1}$, Peter Wild ${ }^{1}$, Leoni A. Kunz-Schughart ${ }^{1}$ Frauke Bataille ${ }^{1}$, Ronald Simon ${ }^{2}$, Guido Sauter ${ }^{2}$, Ferdinand Hofstädter ${ }^{1}$ ${ }^{1}$ Institut für Pathologie, Universität Regensburg, Regensburg, Deutschland

${ }^{2}$ Institut für Pathologie, Universität Basel, Basel, Deutschland

Zielsetzung: Eine Cyclooxygenase-2 (COX-2)-Expression kann die Entstehung von Mammakarzinomen bei Mäusen induzieren und eine COX2-Überexpression wurde in Mammakarzinomen nachgewiesen. Das Ziel dieser Tissue-Mikroarray (TMA)-Studie war, in einer grossen Patientenpopulation zu untersuchen, ob die Expression von COX-2 mit klinischpathologischen Parametern und der Prognose des Mammakarzinoms assoziiert ist. Methoden: An einem TMA wurden insgesamt 2197 Patienten mit primärem Mammakarzinom untersucht. Die COX-2-Expression wurde immunhistochemisch bestimmt (monoklonaler Maus-Antikörper, Cayman, Konzentration 5 $\mu \mathrm{g}$, Mikrowellenvorbehandlung). Die Stärke der COX-2- Expression wurde semiquantitativ ausgewertet $(0-3)$. Ergebnisse: Eine mässiggradige $(2+)$ oder starke $(3+)$ Überexpression von COX-2 wurde in $39,6 \%$ und $9 \%$ der Mammakarzinome nachgewiesen. Eine starke COX-2-Expression war signifikant korreliert mit einem hohen Tumorstadium $(\mathrm{p}=0,001)$ and Tumorgrad (nach Elston und Ellis, $\mathrm{p}<0,0001$ ), aber nicht mit einer lymphogenen Metastasierung. Medulläre Mamma- 
karzinome zeigten häufiger eine starke COX-2-Überexpression $(30 \%$ $\mathrm{p}<0,0001)$. Weiterhin war die starke COX-2-Expression signifikant häufiger in Tumoren mit HER2-Amplifikation, Überexpression von p53 und bcl-2 sowie Negativität für die Hormonrezeptoren ER und PR nachzuweisen (jeweils $\mathrm{p}<0,001$ ). Es fand sich eine deutliche Korrelation zwischen starker COX-Expression und einem kürzeren Gesamtüberleben der $\mathrm{Pa}$ tientinnen $(p=0,0043)$. Schlussfolgerungen: Die starke Überexpression von COX-2 ist häufiger in Mammakarzinomen mit schlechten klinischpathologischen Merkmalen und ist signifikant assoziiert mit einem schlechteren Gesamtüberleben der Patientinnen. Diese Ergebnisse sprechen dafür, dass klinische Studien zur Untersuchung der Effektivität von COX-2-Hemmern in Kombination mit anderen Chemotherapeutika in der adjuvanten Therapie von Hochrisiko-Patientinnen mit Mammakarzinom sinnvoll sind.

\section{V44}

Die Kombination Paclitaxel und der FarnesyltransferaseInhibitor SCH66336 in der Behandlung des metastasierten Mammakarzinoms - Eine Phase I/II Studie

${ }^{*}$ H. -J. Lück', D. Roßner ${ }^{1}, A . A y^{1}$; Ch. Schaff', F. Rinnau ${ }^{1}$, R. Hofmann ${ }^{1}$, W. Lersch ${ }^{2}$, H. Kühnle

${ }^{1}$ Gyn. Onkologie/Brustzentrum MH, Hannover, Deutschland

${ }^{2}$ Essex Pharma, München, Deutschland

In soliden Tumoren ist das ras-Onkogen ein wichtiges Stellglied in der intrazellulären Signaltransduktions- kette. Für die aktive Form des rasProteins ist seine Aktivierung durch Farnesylierung notwendig. In prä -klinischen Untersuchungen wurde ein synergistischer Effekt zwischen Paclitaxel und SCH66336 gezeigt. In dieser Phase-I/II-Studie wurden 19 Patientinnen mit mehrfach vorbehandeltem metastasierten Mammakarzinom mit Paclitaxel $80 \mathrm{mg} / \mathrm{m}^{2}$ wöchentlich und $125 \mathrm{mg}$ Sch66336 bid behandelt. Die Kombination wurde für 12 Wochen gegeben, gefolgt von alleiniger Farnesyltransferase-Inhibitor-Einnahme bis zur Progression. Zurzeit sind 18 Pat. auswertbar für die Toxizität und 14 für das Ansprechen. 6 Pat. sind noch unter Therapie. Medianes Alter der Pat. 51 J. (36-80 J). 17/19 Pat. waren mit Anthrazyklinen und Taxanen vorbehandelt. 8 Pat. waren vor Beginn der Therapie refraktär auf die durchgeführten Chemotherapien. Toxizität: Hämatologisch: Leukopenie Grad III: 5/18; keine febriele Neutropenie, keine Grad III Anämien oder Thrombopenien. NichtHämatologisch: Diarrhoe Grad III/IV: 3/18. Sistieren nach Reduktion des SCH66336 auf $100 \mathrm{mg}$ bid. Außer den bekannten Paclitaxel-assoziierten Toxizitäten wie PNP, Myalgien und Athralgien (keine Grad 2/3), traten keine schweren nicht-hämatologischen Nebenwirkungen auf. Effektivität Eine Pat. ist seit mehr als 12 Mon. in einer CR. 7 Pat. hatten eine PR und 2 Pat. mit minimal Response. Es wurde kein primäres Therapieversagen beobachtet. Die med. Zeit bis zur Progression betrug für Pat. Mit CR+PR 12,2 Monate. Bei Pat. mit einer SD betrug die Zeit bis zur Progression 6,8 Mon. Zusammenfassung: SCH66336 ist in der Lage das Tumorwachstum allein und in Kombination beim metastasierten Mammakarzinom zu beeinflussen. Die auftretenden Toxizitäten sind akzeptabel.Die tägliche Dosis von SCH66336 sollte 2x100 mg nicht überschreiten. Die Aktivität ist vielversprechend und sollte weiter untersucht werden.

\section{V45}

WSG-AM01 Tandem Hochdosischemotherapie (HDC) mit autologer Stammzelltransplantation versus dosisdichte konventionelle Chemotherapie mit G-CSF beim Hochrisiko-Mammakarzinom: Prognostische Faktoren für das Langzeitüberleben

*Ulrike Nitz, Svjetlana Mohrmann, Oleg Gluz, Gerhart Schütt, Frank Werner, Hans-Georg Bender

Westdeutsche Studiengruppe Frauenklinik der Heinrich-Heine Universität Düsseldorf, Düsseldorf, Deutschland

Zielsetzung: Die Dosisintensivierungstrategien und die Hochdosischemotherapie in der adjuvanten Therapie des Mammakarzinoms werden im Moment kontrovers diskutiert. Mit dem Ziel Subgruppen mit maximalem Vorteil von HDC zu identifizieren haben wir retrospektive Analysen durchgeführt. Materialien und Methoden: 403 Patientinnen wurden ran- domisiert. Sie erhielten $4 \mathrm{x}$ EC gefolgt von $3 \mathrm{xCMF} q 2 \mathrm{w}+\mathrm{G}-\mathrm{CSF}$ oder $2 \mathrm{x}$ $\mathrm{EC}$ als Induktion gefolgt von $2 \mathrm{x}$ Hochdosischemotherapie EC+Thiotepa mit autologer Stammzelltransplantation. Die klassischen Prognosefaktoren, wie Alter, Tumorgröße, Grading, Nodalstatus, Hormonrezeptorstatus und Menopausalstatus waren für alle 403 Patientinnen bekannt. Zurzeit finden an Paraffinblöckchen weitere Analysen zur Bestimmung molekularbiologischer Faktoren wie Her-2/neu, Ki-67 und p53 statt. Eine multivariate Analyse wird durchgeführt. Ergebnisse: Bei einer medianen Nachbeobachtungszeit von 48,6 Monaten zeigt sich im Hochdosisarm ein signifikanter Gesamtüberlebensvorteil. Im Rahmen der Studie wurde ein Kollektiv mit einem sehr hohen Rezidivrisiko mit durchschnittlich 17,6 befallenen Lymphknoten behandelt. Laut den vorliegenden (monovariaten) Analysen profitieren insbesonderere junge Patientinnen ( $<35$ Jahre), Patientinnen mit G3 Histologie und solche mit einer Tumorgröße $>4 \mathrm{~cm}$ von der HDC. ZVon der stattfindenden multivariaten Analyse erwarten wir zusätzliche Erkenntnisse. Zusammenfassung: Die retrospektiven Analysen der Prognosefaktoren aus anderen Studien haben interessante Ergebnisse geliefert. Her-2/neu, Alter und Grading sind die bestdokumentierten Prognosefaktoren für das Langzeitüberleben nach HDC. Unsere Daten werden für die Planung zukünftiger Studien von großer Bedeutung sein.

\section{V46}

\section{Taxantherapie in der adjuvanten Behandlung des Hochrisiko- Mammakarzinoms in der ADEBAR-Studie}

${ }^{*}$ Brigitte Rack ${ }^{1}$, Wolfgang Janni ${ }^{1}$, Maja Heinrigs ${ }^{1}$, Doris Augustin ${ }^{3}$, Wolfgang Simon ${ }^{4}$, Nadja Harbeck ${ }^{2}$, Arthur Wischnik ${ }^{5}$, Marion Kiechle2, Harald Sommer ${ }^{1}$, Klaus Friese ${ }^{1}$

${ }^{1}$ I. Frauenklinik der LMU, München, Deutschland

${ }^{2}$ Frauenklinik, Klinikum rechts der Isar der TU München, München, Deutschland

${ }^{3}$ Mammazentrum Oberbayern, Deggendorf, Deutschland

${ }^{4}$ Robert- Bosch- Krankenhaus, Stuttgart, Deutschland

${ }^{5}$ Zentralklinikum, Augsburg, Deutschland

Die ADEBAR-Studie ist eine prospektive multizentrische Phase III Studie, die untersucht, ob Patientinnen mit Hochrisikomammakarzinom ( $\geq 4 \mathrm{LK}$ ) von einem sequenziellen EC-Doc-Regime (E90C-D: 4 Zyklen Epirubicin [E] $90 \mathrm{mg} / \mathrm{m}^{2}$ und Cyclophosphamid [C] $600 \mathrm{mg} / \mathrm{m} 2$ q21d gefolgt von 4 Zyklen Docetaxel [D] $100 \mathrm{mg} / \mathrm{m}^{2}$ q21d) im Vergleich zu einer anthracyclinbasierten Polychemotherapie (FE120C: 6 Zyklen E $60 \mathrm{mg} / \mathrm{m}^{2}$ d 1+8, 5-Fluorouracil $500 \mathrm{mg} / \mathrm{m}^{2} \mathrm{~d} 1+8$ and C $75 \mathrm{mg} / \mathrm{m}^{2} \mathrm{~d} 1-14, \mathrm{q} 4 \mathrm{w}$ ) profitieren. Mit insgesamt 174 aktiven Zentren und einem aktuellen Randomisationsstand von 935 Patientinnen ist die ADEBAR-Studie in dieser Risikogruppe derzeit die am besten rekrutierende Studie in Deutschland. Ziel der vorliegenden Untersuchungen war es, zu überprüfen, ob die Patientenversorgung durch die Teilnahme an der Studie verbessert werden kann. Anhand eines Fragebogens wurden alle registrierten Zentren gebeten, den Einfluss der Studienteilnahme auf ihre eigenen Therapiestrategien und die Patientenversorgung zu erfassen. Insgesamt wurden $67,4 \%$ $(\mathrm{n}=93)$ der Fragebögen beantwortet zurückgesendet. Im Jahr vor Beginn der ADEBAR-Studie hatten 54,8\% der teilnehmenden Zentren ihre Patientinnen ausschließlich außerhalb klinischer Studien behandelt. Dabei hatten $51,7 \%$ dieser Patientinnen vor der Studienteilnahme routinemäBig eine weniger effektive Therapie erhalten. Aufgrund der Teilnahme an der ADEBAR-Studie stellten 43\% der Zentren eine Zunahme der Intensität ihrer Patientenversorgung, und $53,7 \%$ eine Verbesserung ihrer Kenntnisse im Bereich Mammakarzinom fest. Die Teilnahme an klinischen Studien führt, möglicherweise durch die intensivere Betreuung der Patientinnen im Rahmen der Studie und aufgrund regelmäßiger Informationsveranstaltungen und Newsletter für die betreuenden Ärzte, zu einer verbesserten Patientenversorgung in den Zentren. Das konnte durch die Zentren der ADEBAR-Studiengruppe bestätigt werden. 
V47

Phase-II-Study of Gemcitabine (G) as Prolonged Infusion and Vinorelbine (V) as Second-Line Therapy in Metastatic Breast Cancer

*Peter Schmid ${ }^{1}$, Volker Heilmann², Carsten-Oliver Schulz', Annette Dieing ${ }^{1}$, Sylvia Lehenbauer-Dehm ${ }^{1}$, Bernd Flath ${ }^{1}$,

Orhan Sezer ${ }^{1}$, Rolf Kreienberg ${ }^{2}$, Kurt Possinger ${ }^{1}$

${ }^{1}$ Medizinische Klinik mit Schwerpunkt Onkologie und Hämatologie,

Charité Campus Mitte, Humboldt Universität Berlin, Berlin

Deutschland

${ }^{2}$ Universitätsfrauenklinik UIm, UIm, Deutschland

Purpose: $\mathrm{G}$ and $\mathrm{V}$ are active agents for the treatment of metastatic breast cancer. Prolonged infusion of $\mathrm{G}$ can result in higher levels of active metabolites compared to shorter administration. This phase II trial was initiated to evaluate the efficacy and tolerability of the combination as second-line treatment. Patients and Methods: Patients with metastatic breast cancer who had received one prior chemotherapy for metastatic disease were treated with $\mathrm{G}\left(350 \mathrm{mg} / \mathrm{m}^{2}\right.$ as 4 hour infusion $)$ and $\mathrm{V}\left(25 \mathrm{mg} / \mathrm{m}^{2}\right)$ on days 1 and 8 . Treatment was repeated every 3 weeks for up to 6 cycles. Results: 26 patients (median age 58,3 years; visceral metastases $88,5 \%$; prior anthracyclines $76,9 \%$; prior taxanes $46,2 \%$ ) received a total of 111 cycles. Treatment was well tolerated. Predominant toxicity was myelosuppression with grade $3 / 4$ leukopenia and neutropenia in $15,9 \%$ and $11,7 \%$ of courses Non-hematologic toxicity was mild. Most common toxicities were nausea vomiting, peripheral neuropathy and elevation of liver enzymes. One patient developed a paralytic ileus after the first course. No further grade 4 non-hematologic toxicities were observed. Twenty-two patients were assessable for response. Five patients achieved a partial response and 1 patient a complete response accounting for an overall response rate of $27 \%$ In addition, $8(36 \%)$ patients had stable disease. Median duration of response was 7,3 months. Median TTP and overall survival were 4,6 months and 10,5 months, respectively. Conclusion: gemcitabine as prolonged infusion and vinorelbine are a safe and effective treatment in anthracycline and/or taxane pretreated patients.

V48

Die Intraoperative Radiotherapie mittels Elektronen (IOERT) als Boost-Strategie in der BET des Mammakarzinomes: Das Salzburger Konzept

Felix Sedlmayer ${ }^{1 *}$, Christian Menzel ${ }^{2}$, Roland Reitsamer ${ }^{2}$, Walter Cimpoca ${ }^{2}$, Florian Merz ${ }^{1}$, Heinz Deutschmann ${ }^{1}$, Peter Kopp ${ }^{1}$ Gerhard Kametriser ${ }^{1}$, Olaf Nairz ${ }^{1}$, Michael Kopp ${ }^{1}$, Georg Hutarew ${ }^{3}$, Jörg Hutter ${ }^{4}$

${ }^{1}$ Universitätsklinik für Radiotherapie and Radio-Onkologie,

Salzburg, Österreich

${ }^{2}$ Universitätsklinik für spezielle Gynäkologie und Brustambulanz, Salzburg, Österreich

${ }^{3}$ Institut für Pathologie, Salzburg, Österreich

${ }^{4}$ Universitätsklinik für Chirurgie, Salzburg, Österreich

Zielsetzung: Nach brusterhaltender Therapie (BET) treten lokale Rezidive überwiegend im Bereich des ehemaligen Tumorbettes auf. Die Lokalrezidivrate kann durch einen zusätzlichen Tumorbett-Boost nach Ganzbrustbestrahlung gesenkt werden. Mittels intraoperativer Radiotherapie kann das Tumorbett während der Operation mit einer erhöhten Einzeldosis vorweggenommen geboostet werden. Materialien und Methoden: Zwischen 10/98 und 3/04 wurde bei 569 primär konservativ operierten Patientinnen das Tumorbett intraoperativ am Linearbeschleuniger mittels Elektronen bestrahlt (IOERT). Die chirurgischen Ränder wurden im Gefrierschnitt evaluiert, Nachresektionen mittels inverser Telepathologie topographisch gesteuert. Das Gewebe um die Exzisions höhle wurde zur IORT temporär adaptiert, die Distanz zur Brustwand sonographisch zur Tiefendosisberechnung ermittelt. Mittels Elektronenenergien zwischen 4-18 Mev wurde eine Referenzdosis von 9 Gy appliziert (90\%-Isodose; runde Plexiglastuben, 5-8 cm diam.). Das mittlere Behandlungsvolumen betrug 38,3 ml (13,2 SD). Nach abgeschlossener Wundheilung erfolgte eine Ganzbrustbestrahlung mit 51 Gy (duktale Karzinome) bzw. 54,4 Gy (lobuläre Karzinome). Ergebnisse: 28 Patientinnen mit massiv positiven Rändern wurden einer sofortigen sekundären
Mastektomie zugeführt, sodaß sich aktuell 541 Patientinnen nach BET in der Nachsorge befinden. Der Follow-up nach IORT beträgt im Mittel 30,5 Monate (7,2 SD; range 2-66). Die kumulative Rate der Akut- und Spätreaktionen im Bereich des Tumorbettes lag bei 3,9\%. Bislang wurde kein In-Brust-Rezidiv beobachtet. Zusammenfassung: Die IOERT des Tumorbetts kann als vorweggenommener Boost nach konservativer Operation des Mammakarzinomes ohne zusätzliche Morbidität durchgeführt werden und führt zu exzellenten lokalen Tumorkontrollraten. Die Methode erlaubt eine optimale Applikationsgenauigkeit bei kleinen Behandlungsvolumina, die mit hoher Dosishomogenität erfasst werden, sowie eine komplette Hautschonung. Zur Zeit werden prospektiv bei selektierten Patientinnen Dosisreduktionen in der Ganzbrustbestrahlung geprüft.

\section{Freie Vorträge: Ästhetische Mamma-Chirurgie}

\section{V49}

\section{Die Reduktionsplastik im Leistungskatalog der GKV - Krankenbehandlung oder Kosmetik?}

\section{Susanne Bauer}

Medizinischer Dienst der Spitzenverbände der Krankenkassen e.V. Essen, Deutschland

Zielsetzung: Da die Beurteilung der Indikation formverändernder Eingriffe an der weiblichen Brust schwierig ist, bittet die zuständige Krankenkasse den Medizinischen Dienst um Abklärung des Leistungsanspruchs. Der Vortrag legt dar, nach welchen Kriterien die gutachterliche Empfehlung zustande kommt. Material und Methoden: Zur Präzisierung des Krankheitsbegriffes n. SGB V wird auf die Sozialrechtsprechung eingegangen. Außerdem wird über die Ergebnisse einer MDK-internen Arbeitsgruppe berichtet, die die internationale Literatur zum Zusammenhang zwischen Brustgröße und Krankheitswert aufgearbeitet hat. Ergebnisse: Um eine geplante Reduktionsplastik als Krankenbehandlung von einem kosmetischen Eingriff abzugrenzen, müssen krankheitswertige Beschwerden vorliegen. Deswegen ist eine Einzelfallbegutachtung mit körperlicher Untersuchung erforderlich. Anhand der erhobenen Daten wird die vom behandelnden Arzt deklarierte plastisch-chirurgische Operationsindikation erörtert. In der Regel unstrittig sind Indikationen für Eingriffe bei Fehlbildungen, entstellenden Asymmetrien und Korrekturen nach/bei Malignomen. Für die Festlegung eines Mindestresektionsgewichtes zur Rechtfertigung einer gewünschten Reduktionsplastik fand sich in der Literatur keine Grundlage. Es gibt auch keine wissenschaftlichen Arbeiten, die den Vorteil einer Reduktionsplastik gegenüber konservativen Behandlungsmöglichkeiten zur Beschwerdebesserung bei Rückenschmerzen belegen. Der aus diesen Erwägungen resultierende Entscheidungsbaum zur Ableitung einer fundierten sozailmedizinischen Empfehlung wird vorgestellt. Zusammenfassung: Die Beurteilung der Makromastie führt in einen Bereich ärztlichen Handelns, den der Sachverständigenrat als Grauzone bezeichnet hat: das Problem ist allein durch den Rückgriff auf wissenschaftliche medizinische Evidenz nicht lösbar. Der Gutachter ist zu einer sorgfältigen Sachstandsermittlung verpflichtet, die gutachterliche Beurteilung stellt eine Gewissensentscheidung dar.

\section{V50}

\section{Plastische Rekonstruktion von Mammafehlbildungen}

*Jan Esters, Andrea Schulz, I. Herren, Andreas Arens, Jutta Liebau Fachklinik Hornheide, Abteilung Plastische Chirurgie, Münster, Deutschland

Angeborene Mammafehlbildungen stellen eine besondere Herausforderung an die plastische Brustchirurgie dar. Da das Körperbewußtsein der Frau maßgeblich durch ihre Brustform bestimmt ist und die Fehlentwicklung der Brüste in der Pubertät zu starker psychischer Beeinträchtigung führen kann, ist die Intervention für eine plastische Korrektur u.a auch in jungen Jahren zu stellen.

In dem 4-Jahreszeitraum 2000 bis 2003 führten wir in der Fachklinik Hornheide bei 219 Patienten eine Brustkorrektur mit Implantaten durch. Die meisten Korrekturen fielen auf 194 Patienten mit Involutionsatrophie bzw. Mammahypoplasie. 22 Patienten mit tubulärer Fehlbildung bzw. Mammaasymmetrie sowie 3 Patienten mit Poland- bzw. Amazon-Syndrom wurden operiert. 
Die Therapie der tubulären Derformität wurde zweizeitig durchgeführt. In der ersten Operation wurde die Brust subpektoral oder epipektoral augmentiert. Anschließend, in einer erneuten Operation, konnten übergroße Areolae verkleinert, der untere Brustpol neu geformt und die Submammärfalte versetzt werden. Die Areolaverkleinerung dient der Minimierung des Mamillenprolapses.

Kombinierte Brust- und Brustkorbdeformitäten, wie z.B. das Poland- oder das Amazon-Syndrom behandelten wir zunächst mit einer Expanderprothese zur Dehnung des Hautmantels, um anschließend eine dauerhafte Augmentation mittels Kombinationsprothese (z.B. rundes und halbmondförmiges Implantat, MPU-beschichtet) zu erreichen. Alternativ ließen wir bei Patienten mit dem o.g. Syndrom «customer-made»-Implantate anfertigen.

Die Art des Eingriffs hängt von der Brustfehlbildung ab. Von einer einfachen Korrektur der Brustwarzen bis hin zur Formenkorrektur der Brustdrüse und Neumodellierung der Brust mittels Einbringen von Implantaten werden die chirurgischen Kenntnisse des Operateurs gefordert.

Wir stellen standardisierte Vorgehensweisen unserer Klinik zur Diskussion.

V51

\section{The vascularization of capsules around implants depends on} texture and bacterial colonization

*Jian Farhadi' ${ }^{1}$ Franz Schwarzl' ${ }^{1}$, B. Barra ${ }^{1}$, Elisabeth Bruder ${ }^{2}$, Ursula Flückiger ${ }^{3}$, Gerhard Pierer ${ }^{1}$

${ }^{1}$ Department of Plastic and Reconstructive Surgery,

University Hospital, Basel, Switzerland

${ }^{2}$ Institute of Pathology, University Hospital, Basel, Switzerland

${ }^{3}$ Section of Infectious Disease, University Hospital, Basel,

Switzerland

Textured implants have lowered the rate of capsular contracture and infection. The aim of this study was to show the relationship of bacterial colonization and vascularization in textured and smooth implants.

On the back of female albino guinea pig $(n=20)$, a textured and a smooth silicone mini-implant was subcutanously placed. The implants were colonized prior to implantation with either staphylococcus epidermidis with increasing concentrations from $10^{2}$ to $10^{7} \mathrm{cfu} / \mathrm{ml}$ or with staphylococcus aureus in dilution series of $10^{3}$ and $10^{4} \mathrm{cfu} / \mathrm{ml}$. After 2 weeks $(\mathrm{n}=10)$, respectively 4 weeks $(n=10)$ the implants were removed and the capsules were processed for histology (Hämatoxylin-Eosin). Analyses were performed for transverse section of vessels $\left(\mathrm{in} \mathrm{mm}^{2}\right.$ ). The data consists of the mean value of 5 measurements.

The vascularization of textured implants was higher than with smooth implants by the factor 4.2 after colonization with staphylococcus aureus, respectively by the factor 9.2 with staphylococcus epidermidis. Furthermore the amount of vascularization was dependent on the amount of bacterial colonization in textured implants: starting from $102 \mathrm{cfu} / \mathrm{ml} \mathrm{staph}$. epi, the Vascularization was tripled to $10^{7} \mathrm{cfu} / \mathrm{ml}$, and from $10^{3} \mathrm{cfu} / \mathrm{ml}$ staph aureus to $10^{4} \mathrm{cfu} / \mathrm{ml}$ doubled.

This study showed that the amount of vascularization is higher in textured implants than in smooth ones and the amount of vascularization is as well proportional to the bacterial colonization, so therefore an ongoing subclinical infection could be better targeted by textured implants than by smooth ones

\section{V52}

Kosmetische subglanduläre Mammaaugmentation mit runden Silikongelimplantaten

\section{* Siegfried Grandel, Christian Roessing, Anette Görg}

Klinik für Plastische Chirurgie, Hand- und Wiederherstellungs-

chirurgie, Luisenhospital Aachen, Aachen, Deutschland

Vorgestellt wird unsere operative Technik der kosmetischen Mammaaug mentation mit runden texturierten Silikongelimplantaten, welche, auch bei dünnem Hautweichteilmantel, subglandulär eingebracht werden. Es wird auf Risiken und Komplikationen sowie Nachbehandlung in unserem Patientenkollektiv der letzten 7 Jahre eingegangen.

\section{V53 \\ Behandlung der Tuberösen Brust mit dem 'Glandular Flap' nach Egle Muti}

\section{*Hermann Lampe, Marianne Wolters}

Gemeinschaftspraxis für Plastische Chirurgie, Frankfurt, Deutschland

Seit Juni 2002, wo wir auf einem Kongreß in Barcelona die Methode von Muti kennenlernten, haben wir neun Patientinnen mit tuberöser Brust nach dieser Technik operiert. Im November 2003 operierte Egle Muti selbst eine Patientin auf dem III. Frankfurter Live-Operationskurs, bei dem zirka 80 unserer Kollegen diese Operationsmethode kennenlernten. Ein dreieckiger, basal gestielter Drüsenlappen wird umschnitten und nach Tiefersetzen der inframammaren Falte umgeklappt und im unteren Drüsenpol, der bei der tuberösen Brust immer ein Volumendefizit hat, fixiert. Die restliche Drüse wird neugeformt durch Nähte zwischen den verbleibenden lateralen Pfeilern, die mobilisiert wurden. Bei Bedarf kann zusätzlich zur Drüsenneuformung ein Implantat eingebracht werden. Die Methode wird genau dargestellt. Von unseren neun Patientinnen bekamen zwei zusätzlich ein Implantat zur Brustvergrößerung. Die ästhetischen Resultate sind in allen Fällen sehr befriedigend; vor allem die $\mathrm{Pa}-$ tientinnen selbst stuften die Ergebnisse so ein. Die vorher praktizierten Operationstechniken mit einfacher Ausbreitung des retroareolären Drüsengewebes und Verkleinerung der Areola wurden von uns ganz aufgegeben zugunsten der Muti-Technik.

V54

\section{Augmentation mit Mastopexie - Guidelines}

*Anette Meiré, Dirk F. Richter, Mathias Reichenberger, Alexander Stoff, Michael Schneeweiß, Maria Achontaki Abteilung für Plastische Chirurgie -

Dreifaltigkeits-Krankenhaus, Wesseling, Deutschland

Die Augmentation bei verschiedenen Ptosisgraden stellt eine anspruchsvolle planerische und operative Aufgabe dar.

Anhand des eigenen Patientengutes und der gesammelten Erfahrungen sollen Behandlungsempfehlungen aufgezeigt und dargestellt werden.

Zunächst sollte der Grad der Ptosis und die gewünschte Volumenvermehrung analysiert werden. Hierbei sind wichtige anatomische Messpunkte aber auch die Art der Ptosis von entscheidender Bedeutung für das Erstellen der Behandlungsstrategie. Als erforderliche Parameter haben sich bewährt:

Mammillen-Jugulum-Abstand, Mammillendurchmesser, Mammillen-Sternum-Abstand, Unterbrustfaltenlänge und die Volumenbeurteilung der vier Quadranten. Die Mammillen-Projektionshöhe im Stehen gemessen ist ebenfalls für die Planung hilfreich.

Je nach Befunderhebung stehen neben der reinen «inneren Straffung» durch prothetische Volumenvermehrung alle anderen Methoden, wie die periareoläre Straffungsfigur, der I-Schnitt, aber auch der inverse T-Schnitt zur Verfügung.

Je nach Ausgangsbefund ist zusätzlich die Wahl des Prothesenlagers auf oder unter der Brustmuskulatur zu klären und ob wegen der exponentiellen Risikovermehrung ein zweizeitiges Vorgehen in Betracht gezogen werden sollte.

Unsere Erfahrungen haben ein großzügiges Vorgehen hinsichtlich der Straffungsfiguren gezeigt. Bei grenzwertigen Befunden sollte man sich lieber für das nächst aufwendiger auch narbenreichere Verfahren entscheiden, um ein besseres Resultat erzielen zu können.

Dies gilt insbesondere bei Korrektur-Operationen, was anhand unseres umfangreichen Patientengutes dargestellt werden soll. 
V55

Die Mammareduktionsplastik - die Vertikalnaht im Vergleich zum Inversen T

*Tim Middelberg, Andrea Schulz, Andreas Arens, Jutta Liebau Fachklinik Hornheide, Abteilung Plastische Chirurgie, Münster, Deutschland

Die Mammareduktionsplastik bei Makromastie gehört zum klassischen mammachirurgischen Spektrum der Plastischen Chirurgie. Mit einfachen und standardisierbaren Operationsmethoden lassen sich vorhersagbar gute und symmetrische Reduktionsergebnisse erzielen. Die Auswahl an beschriebenen Verfahren zur Brustverkleinerung ist groß. Die Mammareduktionsverfahren lassen sich in Techniken mit einer vertikalen Narbe und Techniken mit einer invers T-förmigen Narbe submammilär zusammenfassen.

Zum Erfolg der Operationstechnik führt bei der Mammareduktionsplastik die richtige Indikationsstellung. $\mathrm{Zu}$ unterscheiden ist die unproportional große Brust verglichen mit dem Habitus der Patientin von einer Mammahyperplasie bei allgemeiner Adipositas und von der Ptosis mammae. Die Indikation zur Mammareduktion bei der Mammahyperplasie als wahre Fehlbildung ist eine medizinische und wird in vielen Fällen von den Krankenversicherungen übernommen.

Die Wahl der Operationstechnik hängt vor allem vom zu erwartende Resektionsgewicht ab. Eine Vertikalnaht ist bei kleineren Resektionsgewichten anzustreben, große Resektionsvolumina sind der Operationsmethode mit einem Inversen T-Schnitt vorbehalten

Die Technik der vertikalen Mammareduktionsplastik wurde von Madelaine Lejour beschrieben. Es bestehen einige Modifikation der klassischen Lejour-Technik.

Die Reduktionstechnik mit einer invers T-fömigen Narbe ist die klassische Methode. Sie wurde von Wise, Strömbeck und Pitanguy geprägt. S Tabelle. Die allgemeinen Operationsrisiken sind Nachblutung, Infektion, Hämatom, Serom, Wundheilungsstörungen und hypertrophe bzw. dehiszente Narbenbildung.

Als spezielle Operationsrisiken lassen sich die Asymmetrie der Mammae, eine Dog-Ear-Bildung, die Verlust der Laktationsfähigkeit, Sensibilitätsverlust oder Nekrose der Mamillen und Fettgewebsnekrosen benennen. Die Mammareduktionsplastik nach den oben beschriebenen Methoden ist standardisierbar, reproduzierbar und führt zu ordentlichen Reduktionsergebnisse. Große revisionsbedürftige Komplikationen sind sehr selten. Kleine Nachbesserungen wie Narben- oder Dog-Ear-Korrekturen lassen sich oft sogar in Lokalanästhesie durchführen. Die Patientin mit einer Mammahyperplasie leidet oft extrem unter dieser Fehlbildung und is durch einen hohen Leidensdruck bewegt. Die Patientenzufriedenheit nach Erfolg der Operation ist entsprechend hoch.

Vertikalnahl vs. Inverses T

\begin{tabular}{lll}
\hline & Vertikalnaht & Inverses T \\
\hline Vorteile & narbensparend & - für alle Resektions- \\
& - reproduzierbar gute & gewichte geeignet \\
& Ergebnisse & - reproduzierbar gute \\
- hohe & Ergebnisse \\
Patientezufriedenheit & - primäres OP-Ergebnis \\
- Sensibilitätsverlust der & näher am endgültigen \\
Mamillen selten & Resultat \\
& & - hohe Patientenzufrieden- \\
& heit \\
\hline Nachteile & begrenztes & - Wundheilungsstörungen \\
& Resektionsgewicht & im T der Narbe \\
- primäres OP-Ergebnis & - zusätzliche Narbe in der \\
entspricht nicht dem & Submammarfalte \\
endgültigen Resultat & - laterale Dog-Ear-Bildung \\
- korrekturwürdige Narbe & - Sensibilitätsverlust der \\
kaudal über die & Mamillen höher \\
Submammarfalte hinaus- & - Laktationsunfähigkeit \\
reichend & \\
\hline
\end{tabular}

V56

\section{Endoskopieassistierte submuskuläre Brustaugmentation}

*Arash Momeni, Matthias Föhn, Nestor Torio, Holger Bannasch, Jörg Borges, G. Björn Stark

Abteilung für Plastische und Handchirurgie, Universitätsklinikum Freiburg, Freiburg, Deutschland

Seit 1997 bieten wir als von den Patientinnen frei wählbare Alternative zum inframammären Zugang die ambulante transaxilläre Augmentation mit im allgemeinen runden texturierten Vollgelprothesen. Die Implantation erfolgt grundsätzlich epithorakal, d.h. im kranialen Anteil submuskulär. Dieser Zugang wird von 51,6\% der Patientinnen bevorzugt. Die endoskopische Dissektion des kaudalen Implantatlagers mit Durchtrennung der inferioren sternokostalen Muskelursprünge erlaubt eine komplikationsarme und exakte Implantation. Patienten und Technik: Von 1997 2004 wurden überwiegend aus rein ästhetischer Indikation bei 91 Patientinnen eine Augmentation durchgeführt (181 Prothesen). Bei 12,1\% der Patientinnen lag zusätzlich zur bestehenden beidseitigen Mammahypoplasie eine Ptose vor, welche in 2 Fällen durch eine zusätzliche Straffung behandelt wurde. In letzterem Fall erfolgte die Prothesenimplantation über die Resektionsinzisionen. Die Prothesenvolumina lagen zwischen 150 und $500 \mathrm{ml}$. In 51,6\% der Fälle erfolgte die Implantation endoskopieassistiert transaxillär und in 45,1\% inframammär offen. Ergebnisse: Es traten nur $2(=2,2 \%)$ revisionsbedürftige Frühkomplikationen in Form von Hämatomen auf; beide beim offenen inframammären Zugang. In beiden Fällen wurde das Operationsergebnis nicht beeinträchtigt. Beobachtete Spätkomplikationen waren: einseitige Dislokationen (5,5\%), Kapselkontrakturen $(1,1 \%)$ sowie tastbare Faltenbildungen $(1,1 \%)$. Nach 6 Monaten ist die Narbenbildung im allgemeinen nicht sichtbar. Schlussfolgerung: Die endoskopieassistierte transaxilläre, epithorakale (partiell submuskuläre) Augmentation mit runden texturierten Prothesen stellt eine sichere und ästhetisch voraussehbare Technik dar. Die axilläre Schnittführung wird vom Großteil der Patientinnen bevorzugt. Die operativ-technischen Details werden im Vortrag dargestellt.

\section{V57}

\section{Gynäkomastie}

${ }^{*}$ Andrea Schulz, I. Herren, Jutta Liebau

Fachklinik Hornheide, Abteilung für Plastische Chirurgie, Münster, Deutschland

Im Zeitraum von 24 Monaten zwischen 6/2002 und 6/2004 wurden $96 \mathrm{~Pa}-$ tienten mit einer Gynäkomastie in der Fachklinik Hornheide, Münster operativ behandelt.

Am Anfang jeder Behandlung steht die differentialdiagnostische Abklärung folgender Krankheitsbilder: Pubertätsgynäkomastie, Pseudogynäkomastie oder Lipomastie, Gynäkomastie bei endokrinen Störungen, medikamentös induzierte Gynäkomastie und bei chronischen Erkrankungen (z.B. Leukose, Urämie, Leberzirrhose). Die Gynäkomastie beruht letztlich immer auf einem Überwiegen der das Wachstum der Brustdrüse stimulierenden (Östradiol und Prolaktin) gegenüber den inhibitorischen Faktoren (vor allem Testosteron). Sie ist also nicht als eigenständiges Krankheitsbild sondern vielmehr als ein Syndrom aufzufassen. Therapeutische Schritte richten sich nach der Ursache. Die Pubertätsgynäkomastie bildet sich in der Regel spontan zurück. In ausgeprägten Fällen mit fehlender Prognoseverbesserung oder starkem Leidensdruck wird in Abhängigkeit von den anatomischen Gegebenheiten eine subcutane Mastektomie mit angleichender Liposuktion durchgeführt. Bei resultierendem Hautüberschuß oder vorbesteh

endem Mamillenprolaps wird dieses Vorgehen durch eine konzentrische Hautmantelstaffung mit einem nicht resorbierbaren Faden ergänzt. Postoperativ wird ein Kompressionsmieder für 6-12 Wochen generell empfohlen. 
V58

Transareolärer Zugang zur ästhetischen Mammaaugmentation

*Katrin Seidenstücker, Bianca Baican, Klaus Exner

Markus-Krankenhaus, Frankfurt am Main, Deutschland

Operative Zugangswege der Mammaaugmentation müssen verschiedene Kriterien berücksichtigen: unauffällige Narben, Gewebeschonung, übersichtliche Präparation. Problematische Weichteilverhältnisse erfordern die Präparation unter Sicht, die der ästhetisch günstige axilläre Zugang nicht bietet. Der einfache, häufig angewendete inframammäre Zugang hinterlässt sichtbare und stigmatisierende Narben, ebenso der periareo-läre Zugang.

Bei Dermatochalasis, extremer Involutionshypoplasie, angeborener Asymmetrie und Entwicklungsstörungen favorisieren wir einen transare olären, peripapillären Zugang, der eine kaum sichtbare Narbe innerhalb der Areola hinterlässt. Bei der Dissektion des Drüsenkörpers werden die zentralen Milchgänge geschont. Die Pektoralisfaszie wird dargestellt und das Implantatlager subfaszial scharf präpariert. Perimysium, Faszie, Drüsenkörper, Fettgewebe und Dermis bilden die ventrale Weichteildeckung des Implantates. Eine rein subkutane Implantatlage wird verhindert. Diese OP-Technik erlaubt auch eine subpektorale Implantatlage.

Seit 1996 haben wir diese OP-Technik bei 72 Patientinnen angewandt und Implantate zwischen $120 \mathrm{cc}$ und $340 \mathrm{cc}$ eingebracht. Redondrainagen wurden für 24 Stunden eingelegt. Die Schnitt-Nahtzeit betrug im Mittel 47 Minuten, bei simultanem, beidseitigem Vorgehen.

Bei der Beurteilung der Ergebnisse wurden folgende Parameter evaluiert: die Mamillensensibiltät in der subjektiven Wahrnehmung der Patien tin, die Qualität der Narbe, die Symmetrie der augmentierten Brust, postoperative Komplikationen und Spätfolgen. Die schwerste Komplikation war ein postoperatives Hämatom mit folgender Kapselfibrose, die einen Sekundäreingriff erforderlich machte.

Der transareoläre, peripapilläre Zugang ermöglicht auch bei problematischen Weichteilverhältnissen eine übersichtliche Präparation, über die die Mammaimplantate optimal positioniert werden können. Die guten Ergebnisse rechtfertigen die Anwendung dieser Operationstechnik. Die Nachteile der Dissektion des Drüsenkörpers werden durch die verbesserte Weichteildeckung bei subfaszialer Implantatlage aufgewogen.

\section{V59}

\section{Axilläre Mammaaugmentation: Tipps, Tricks - spezielle Instrumente}

\section{Hermann Solz, *Henning Ryssel}

Mannheimer Klinik für Plastische Chirurgie, Mannheim, Deutschland Die operative Brustvergrößerung ist weltweit einer der häufigsten Eingriffe in der ästhetisch-plastischen Chirurgie.

Bei der Optimierung der Brustform ist darauf zu achten, dass die Narben möglichst unauffällig sind und eine natürliche Formgebung erfolgt. Vor allem unschöne und sichtbare Narben limitieren das Ergebnis einer Brustvergrößerung sehr.

Der Vorteil der axillären Schnittführung ist die Gestaltung einer unauffälligen Narbe fern ab der Brust als eigentliches Zielorgan. Der Brustdis sektors nach Solz gewährleistet sowohl für die subglanduläre, als auch die submuskuläre Präparation eine Schonung des Gewebes und ein Respektieren der anatomischen Strukturen. Durch die Bajonettform des Dissektors kann nach initialer manueller Präparation die Imlantatloge atraumatisch geformt werden. Als Resultat zeigt sich eine homogene Implantat loge unter Berücksichtigung der anatomischen Strukturen. Die stumpfe Präparation mit dem Dissektor bringt den Vorteil, dass es nahezu keine Blutungen gibt. Dies konnten wir in einer Statistik von 96 Brustvergrößerungen durch Bestimmung der Drainagenvolumina deutlich zeigen.

Eine Vergrößerung der Narbe durch Ausreissen der Schnittränder während der Implantateinbringung ist unbedingt zu vermeiden, da dies das ästhetische Resultat erheblich verschlechtert. Durch eine spezielle narbenminimierende Naht kann dies verhindert werden.

Wir sehen dieses Vorgehen als ideale Standardisierung für die ästhetische Brustvergrößerung.

Schlussfolgerung: Die axilläre Schnittführung ist ideal, um eine unauffällige Narbe zu gestalten, die Brust selbst wird mit dieser Methode nicht tangiert. Durch die Präparation mit dem Dissektor nach Solz werden Forderungen wie atraumatische Präparation, Respektieren der anatomischen Strukturen, Reduktion des Blutungsrisikos erfüllt.
V60

Erfahrungen mit anatomischen Implantaten in der ästhetischen Mammachirurgie bei 53 Patientinnen

Matthias Voigt

Praxis für Plastische Chirurgie Freiburg, Freiburg, Deutschland

In der ästhetischen Mammachirurgie werden vor allem runde Implantate eingesetzt. Seit einigen Jahren sind anatomisch geformte Implantate auf dem Markt, die eine natürlichere Brustform nach der Augmentationsplastik erzielen sollen.

In der Zeit von Juli 2002 bis Mai 2004 wurden bei 53 Patientinnen anatomisch geformte Implantate partiell submukulär über einen submammären Zugang ( $\mathrm{n}=48$ Patientinnen) und einen periareolären Zugang $(\mathrm{n}=5 \mathrm{~Pa}$ tientinnen) eingelegt. Die Größenbestimmung der Implantate erfolgte nach einem modifizierten Tebbets Algorithmus, d.h. als entscheidende Größe für die Implantatauswahl gilt die zur Verfügung stehende Brustbasis. Die Implantatgröße kann dadurch vor der Operation eindeutig festgelegt werden, Probeimplantate wurden nicht verwendet. Die gesamte Präparation wurde mit monopolarem Strom durchgeführt, es wurde keine stumpfe Präparation angewendet. Die Größe der Implantattasche wurde der Implantatgröße angepasst und exakt präpariert.

Es konnte bei allen Patientinnen eine hohe Zufriedenheit durch die erzielte Brustform erreicht werden. Implantatdrehung konnte keine festgestellt werden. Bei 2 Patientinnen kam es zu einer revisionsbedürftigen Nachblutung, die das ästhetische Endergebnis nicht beeinflusste. Wundheilungsstörungen wurden nicht beobachtet.

Die Verwendung von anatomischen Implantaten unterstützt bei korrekter Platzierung eine natürliche Brustform, v.a. wenn sie partiell submuskulär eingelegt wurden. Die exakte Präparation der Implantattasche entsprechend der Implantatgröße vermindert Dislokationen wie Drehungen der Implantate. Durch das Angebot von verschiedenen Implantatgrößen und -abmessungen kann ein individuell passendes Implantat mit der Patientin vor der Operation festgelegt werden und macht die Verwendung von Probeimplantaten meistens überflüssig.

\section{Freie Vorträge: Diagnostik}

\section{V61}

\section{Gensignaturen zur Identifizierung nodal-positiver Mammakarzinome}

*Edgar Dahl1,2, Glen Kristiansen², Klaus Hermann³, Irina Klaman³, Christian Pilarsky ${ }^{3}$, André Rosentha/ ${ }^{3}$, Peter-Johannes Wild ${ }^{4}$, Arndt Hartmann ${ }^{4}$

${ }^{1}$ Institut für Pathologie, Universitätsklinikum der RWTH Aachen, Aachen, Deutschland

${ }^{2}$ Institut für Pathologie, Universitätsmedizin Charité, Berlin, Deutschland

${ }^{3}$ metaGen Pharmaceuticals i. L. Berlin, Deutschland ${ }^{4}$ Institut für Pathologie, Universität, Regensburg, Deutschland

Zielsetzung: Die Therapie des Mammakarzinoms hängt entscheidend vom Nodalstatus der Patientin ab. Dieser wird zunehmend durch die Analyse des Sentinel-Lymphknoten bestimmt. Gensignaturen des Primärtumors, die den Nodalstatus mit hoher Sensitivität voraussagen können, wären sehr hilfreich für die Wahl der optimalen Therapieform. Material und Methoden: Mittels Affymetrix Gene Chip Technologie wurden Genprofile von 47 mikrodissektierten primären Mammakarzinomen und 24 normalen Drüsengeweben der Mamma erstellt. Nach der Normalisierung wurden zirka 300 Probesets selektiert, die sowohl in Tumor- als auch Normalgewebe exprimiert werden (Mindestexpressionshöhe) und ein signifikantes «Signal-Rauschen-Verhältnis» aufweisen. Aus dieser Teilmenge wurden jeweils 10000 Gensignaturen mit $\mathrm{i}=1$ bis 20 Probesets zufällig gezogen und für jedes i die «besten» Signaturen mittels K-Nearest-Neighbour-Verfahren und Kreuzvalidierung (Leave-one-out-Methode) auf einer Trainingsstichprobe von pN1 und pN0 Tumoren ermittelt und schließlich noch auf einer unabhängigen Teststichprobe geprüft. Ergebnisse: Es wurden insgesamt 19 Gensignaturen mit einer Länge von bis zu 20 Genen identifiziert, die sowohl im Training- als auch im unabhängigen Testset die Lymphknoten-Metastasierung mit einer Genauigkeit von 
mehr als $85 \%$ vorhersagen konnten. Zusammenfassung: Es scheint möglich zu sein Gensignaturen zu definieren, mit deren Hilfe sich nodal-positive und nodal-negative Mammakarzinome bereits anhand der Expressionsprofile des Primärtumors unterscheiden lassen. Eine dahingehende molekulare Diagnostik am primären Mammakarzinom könnte den Sentinel-Befund unterstützen. Eine verbesserte Diagnostik sollte insbesondere bei der Therapiewahl bei Patientinnen mit pT1 Tumoren von Bedeutung sein. Wir haben begonnen «kurze» Gensignaturen an einem Gewebe-Microarray mit 300 Mammakarzinomen mittels immunhistochemischer Analysen und RNA in situ Hybridisierung zu bestätigen.

\section{V62}

\section{Wie genau kann die klinische Untersuchung das Ansprechen auf primär systemische Chemotherapie beim Mamma- Carcinom vorhersagen?}

*Uta Euler ${ }^{1,3}$, Joke Tio 1, 4, H. Volkholz² , Agustinus H. Tulusan ${ }^{1}$

${ }^{1}$ Frauenklinik, Bayreuth, Deutschland

${ }^{2}$ Abteilung für Pathologie, Klinikum Bayreuth, Bayreuth,

Deutschland

${ }^{3}$ Abteilung für Gynäkologie u. Geburtshilfe, Krankenhaus München Schwabing, München, Deutschland

${ }^{4}$ Abteilung für Gynäkologie u. Geburtshilfe der Universitätsklinik Münster, Münster, Deutschland

Zielsetzung: Primär systemische Therapie ist heutzutage eine wichtige Therapieoption in der Behandlung des lokal fortgeschrittenen MammaCarcinoms. Ein wesentlicher Vorteil ist die mögliche Beurteilung des Ansprechens vor der Operation. Methoden: Bei 165 Patientinnen mit lokal fortgeschrittenem Mamma-Carcinom wurden eine klinische Untersuchung, eine Mammographie und Ultraschall vor und nach der primär systemischen Therapie mit 3 Zyklen Epirubicin $\left(120 \mathrm{mg} / \mathrm{m}^{2}\right)$ und Cyclophophamid $\left(600 \mathrm{mg} / \mathrm{m}^{2}\right)$, appliziert alle 2 oder 3 Wochen, vor der Operation durchgeführt. Das klinische Ansprechen wurde mit den histologischen Ergebnissen nach der Operation verglichen. Der Grad des klinischen Ansprechens beinhaltete komplette Remission (CR), partielle Remission (Reduktion der Größe und/oder Dichte) (PR), keine Veränderung (NC) oder Fortschreiten der Erkrankung (PD). Ergebnisse: Bei 149 auswertbaren Patientinnen wurde das klinische Ansprechen auf die Chemotherapie als eine komplette Remission in $13,4 \%$, partiell in $73,1 \%$, keine Veränderung in $12,7 \%$ und als weiter fortschreitende Erkrankung in 0,6\% beurteilt. Bei der histologischen Untersuchung nach der Operation konnte be 14 von 20 als klinisch komplette Remission beurteilten Patientinnen noch Resttumor nachgewiesen werden. Die Sensitivität lag bei $89 \%$, die Spezifität bei $75 \%$. Zusammenfassung: Die klinische Untersuchung kann neben bildgebenden Verfahren wie Mammographie und Ultraschall verwendet werden, um das Ansprechen auf primär systemische Therapie zu beurteilen. Obwohl die Sensitivität hoch ist, ist dieses Verfahren nicht akkurat genug, um histologisches Ansprechen eindeutig vorherzusagen.

\section{V63}

Magnetresonanztomographie und Knochenszintigraphie bei Achsenskelettmetastasierung beim Mammakarzinom

${ }^{*}$ Nadir Ghanem ${ }^{1}$, Carsten Altehoefer ${ }^{1}$, Almut Einert ${ }^{1}$, Elmar Kotter ${ }^{1}$, Thomas Kelly², Ingo Brink2 ${ }^{2}$, Mathias Langer ${ }^{1}$

${ }^{1}$ Abt. Roentgendiagnostik, Radiologische Universitätsklinik Freiburg, Freiburg, Deutschland

${ }^{2}$ Abt. Nuklearmedizin, Radiologische Universitätsklinik Freiburg, Freiburg, Deutschland

Zielsetzung: Vergleich der diagnostischen Wertigkeit der MRT und der Knochenszintigraphie (KS) in der Detektion einer Achsenskelettmetastasierung bei Patientinnen mit Mammakarzinom. Materialien und Methodik: Retrospektive Studie an 170 Patientinnen mit Mammakarzinom, bei denen eine Knochenszintigraphie mit 99m Tc-DPD im Rahmen eines Tumorstagings und eine MRT des Achsenskeletts (T1-SE-,T2-TSE-, TIRMSequenzen) zum Staging, Abklärung von neurologischen Beschwerden und Knochenschmerzen und im Verlauf bei bekannter Metastasierung durchgeführt wurde. Ergebnisse: 45/170 (26\%) Patientinnen waren frei von Achsenskelettmetastasen. Bei 90/170 (53\%) Patientinnen lagen mit beiden Verfahren Metastasen am Achsenskelett vor. Somit ergab sich eine Konkordanz von $77 \%$. Bei den konkordant positiven Befunden zeigte die MRT in 46/90 (51\%) ausgedehntere Befunde am Achsenskelett als die Knochenszintigraphie mit therapeutischer Konsequenz (Radiatio, OP). Die KS wies hingegen in 41/90 (46\%) Patientinnen mit positiv konkordanten Befund peripheren Knochenmeta-stasen nach. Die MRT lieferte entscheidende Zusatzinformation 70/90 (78\%). In 35/170 (19\%) lagen diskordante Befunde vor, wobei die MRT in allen Fällen eine ossäre Metastasierung am Achsenskelett nachwies. In 2 Fällen wies die KS periphere Metastasen bei unauffälligem Achsenskelett auf. Zusammenfassung: Im Vergleich mit der KS als Screening-Methode für das gesamte Skelettsystem erwies sich die MRT der Wirbelsäule als das sensitivere Verfahren. Ob schnelle MRT-Bildgebung des Achsenskeletts die KS ersetzen mag, wird sich in prospektiven Studien zeigen. Die MRT ist das Verfahren der Wahl bei lokalen und neurologischen Symptomen.

\section{V64}

\section{Ganzkörper-MRT in der Detektion von Skelettmetastasen im Vergleich zur Skelettszintigraphie bei Mammakarzinompatientinnen}

*Nadir Ghanem 1 , Thorsten Bley', Thomas Kelly², Ingo Brink2, Almut Einert ${ }^{1}$, Elmar Stickeler ${ }^{3}$, Elmar Kotter ${ }^{1}$, Mathias Langer ${ }^{1}$ ${ }^{1}$ Abt. Roentgendiagnostik, Radiologische Universitätsklinik Freiburg, Freiburg, Deutschland

${ }^{2}$ Abt. Nuklearmedizin, Radiologische Universitätsklinik Freiburg, Freiburg, Deutschland

${ }^{3}$ Abt. Frauenheilkunde, Universitätsfrauenklinik Freiburg, Freiburg, Deutschland

Zielsetzung: Vergleich der diagnostischen Wertigkeit der GanzkörperMRT (GK-MRT), Skelettszintigraphie (SKZ) in der Detektion von ossären Metastasen beim Mammakarzinom. Materialen und Methoden: Prospektive Studie unter Einfluss von 40 Mammakarzinompatientinnen, bei denen eine Ganzkörper-MRT (1,5 T Magnetom Sonata, Siemens, Erlangen, Germany) mit einer rollenden Body-SURF-Coil unter Verwendung einer coronaren TIRM-Sequenz für verschiedene Körperregionen im Rahmen des Stagings von Skelettmetastasen angefertigt wurden. Die Skelettszintigraphie mit $99 \mathrm{mTc}$-DPD wurde zeitgleich durchgeführt. Ergebnisse: In 33/40 (82\%) Fälle lagen konkordante Befunde der GK-MRT und SKZ vor, wobei in 15/40 (37\%) Fälle beide Verfahren Skelettmetastasen ausschloßen. Bei 18/40 (45\%) Patienten zeigten GK-MRT und SKZ Skelettmetastasen, wobei in 12/18 (67\%) die GK-MRT ausgedehntere Befunde nachwies. In 2/18 (11\%) war die SKZ der GK-MRT überlegen. In 7/40 $(18 \%)$ lagen diskordante Befunde vor, wobei die GK-MRT in 6 Fällen Skelettmetastasen detektierte bei negativer SKZ. In 1 Fall war die GKMRT falsch negativ, wobei die SKZ eine Humerusmetastase detektierte. In 25/40 (63\%) Patientinnen konnte die GK-MRT tumorassozierte $\mathrm{Zu}$ satzbefunde erheben. Zusammenfassung: Die GK-MRT als ScreeningUntersuchungsverfahren für das gesamte Skelettsystem ist sensitiver. Die GK-MRT ist ein schnelles valides Untersuchungsverfahren zur Detektion von ossären Metastasen und sollte daher in Zukunft eingesetzt werden.

\section{V65}

\section{Abklärung auffälliger Befunde im Mammographie Screening} durch minimal invasive Biopsie

*Hans Junkermann ${ }^{1}$, Ingeborg Junkermann ${ }^{1}$, Markus Borowski ${ }^{1}$, Peter Hanisch ${ }^{2}$, Ulrich Bonk ${ }^{2}$, Werner Böcker ${ }^{3}$

${ }^{1}$ Mammographie Screening, Bremen, Deutschland

${ }^{2}$ Pathologisches Institut des Klinikums-Mitte, Bremen, Deutschland ${ }^{3}$ Pathologisches Institut der Universität, Münster, Deutschland

Zielsetzung: Die Ergebnisse der routinemässigen Anwendung der minimal invasiven Biopsie und ihr Beitrag zur Erfüllung der Europäischen Leitlinien im Mammographie Screening Programm Bremen sollen überprüft werden. Material: Seit Projektbeginn im Juni 2001 bis zum 31,03 2004 wurden 37574 Frauen untersucht (34604 Erstrundenteilnehmerinnen, ERT; 2967 Zweitrundenteilnehmerinnen, ZRT). Bei 5,6\% der ERT und 4,5\% der ZRT wurde bei der Doppelbefundung ein abklärungsbedürftiger Befund erhoben. Nach klinischer Untersuchung, Mammogra- 
phie und Ultraschall wurde bei 591 ERT $(=1,7 \%)$ und 36 ZRT $(=1,2 \%)$ eine histologische Abklärung indiziert. Ergebnisse: Die Gewebeentnahme erfolgte bei 629 Läsionen durch eine minimal invasive Biopsie. Nur bei 45 Läsionen wurde aus verschiedenen Gründen primär eine offene Biopsie durchgeführt. Von den 629 minimal invasiv abgeklärten Läsionen waren $51 \%$ maligne. 359 Läsionen $(=57 \%)$ wurden sonographisch lokalisiert und biopsiert, davon waren $68 \%$ maligne. Von 140 stereotaktisch gezielten Stanzbiopsien waren 34\% maligne und 22\% von 130 stereotaktisch gezielten Vakuumbiopsien. Der routinemässige Einsatz minimal invasiver Biopsien ermöglichte in 93\% der malignen Läsionen eine präoperative Diagnose (EU-Standard optimal $>90 \%$ ) und einen niedrigen Anteil offener Biopsien mit gutartigem Ergebnis (gutartige:bösartige Operationen $=0,27: 1$; EU-Standard optimal $<0,5: 1$ ). Zusammenfassung: Durch den routinemäßigen Einsatz der minimal invasiven Biopsie be allen verdächtigen Mammographiebefunden, die sich nach bildgebender Abklärung nicht eindeutig gutartig zeigten, konnten die EU-Standards bzgl. optimaler präoperativer Karzinomdiagnose bei einer geringen Anzahl offener Biopsien mit gutartigem Ergebnis eingehalten werden.

\section{V66 \\ Nachweis von DCIS und ductalem Karzinom durch Proteinprofiling (SELDI) des Serums}

${ }^{*}$ Christine Koenig ${ }^{2}$, Ivo Meinhold-Heerlein ${ }^{1}$, Christoph Mundhenke ${ }^{1}$ Martin v. Bergen ${ }^{2}$, Gesa Fuhrmann ${ }^{3}$, Walter Jonat ${ }^{1}$, Nicolai Maass ${ }^{1}$

${ }^{1}$ Uni-Frauenklinik, Kiel, Deutschland

${ }^{2}$ LifeLines $\mathrm{GmbH}$, Husum, Deutschland

${ }^{3}$ Strathmann Biotec GmbH, Hamburg, Deutschland

Unterschiedliche Mammatumoren können auf RNA und Proteinebene nachgewiesen werden.Eine Methode zum DCIS Screening besteht bislang nicht. Diese Studie differenziert Serumproben gesunder Frauen und Patientinnen mit benignen, prämalignen und malignen Erkrankungen der Brust durch Proteinprofiling. Zusätzlich wird der Nutzen von SELDI-MS (surface-enhanced laser desorption ionisation- mass spectroscopy) für eine DCIS Früherkennung untersucht.

SELDI-MS ermöglicht quantitative Messungen komplexer Proteingemische, wie des Blutserums. Nach ihren Bindungseigenschaften wurden Proteine angereichert und auf separaten kationischen Proteinchips massenspektroskopisch untersucht.Aus den erhaltenen Proteinprofilen werden Algorithmen gebildet um das Expressionsmuster mit dem Probentyp zu korrelieren. Blutproben von 112 Frauen sind analysiert worden. Als Kontrolle diente Serum von 28 gesunden Frauen. Serumproben von $27 \mathrm{~Pa}$ tientinnen mit gutartigen, 25 DCIS Patientinnen und 32 Patientinnen mit ductalen Mammakarzinomen wurden analysiert.

Die SELDI Analyse zeigte eine Gruppe 12 verschiedener Biomarker. Die Molekülmassen lagen bei 3000-15000 Da. Die Daten wurden in eine Trainings- und eine Testgruppe unterteilt. Der Algorithmus wurde aus verschiedenen Kombinationen von vier Biomarkern erstellt. SELDI zeigt eine gute Unterscheidung zwischen benignen, DCIS und invasiven Läsionen und den gesunden Kontrollen. Es findet sich eine hohe Sensitivität von 0,89 und 0,88 für den Nachweis von DCIS und ductalen Karzinomen im Serum. Der prädiktive Wert für DCIS liegt bei über $80 \%$.

Die SELDI Technik hat ihren Nutzen zur Identifikation unterschiedlich exprimierter Proteine aus Serum von Frauen mit Brustläsionen bewiesen Die hohe Prädiktion von fast $90 \%$ für das DCIS unterstreicht die Bedeutung dieser neuen Methode. Zukünftig könnte SELDI ein wichtiger Bestandteil eines Brustkrebs- bzw. DCIS- Screenings sein.
V67

Qualitätssicherung in der Mammographie durch Javabasiertes Test- und Lehrprogramm in Verbindung mit einer SQL-Datenbank

Nenad Kostovic ${ }^{1},{ }^{*}$ Renate Tewaag ${ }^{1}$, Klaus Dröder ${ }^{2}$

${ }^{1} T$ herapeutisch-Diagnostisches Zentrum, Solingen, Deutschland

${ }^{2}$ Mac Software Design, Münster, Deutschland

Zielsetzung: Entwicklung eines offenen und beliebig erweiterbaren, plattformübergreifenden Test- und Lehrprogramms für die Qualitätssicherung in der Mammographie. Materialien und Methode: Entwicklung eines DICOM-Javaviewers, der erstmalig die willkürliche und durch ein programmeigenes Koordinatensystem gesteuerte Segmentierung der Bilder ermöglicht und so die Hinterlegung von Informationen zu jeweiligen Bildsegmenten erlaubt. In einer gekoppelten SQI-Datenbank werden die Bild- und Befundinhalte zu zirka 300 eingespeisten Mammographien eingegeben. Die Datenbank wurde entsprechend den Test- und Auswertekriterien der KV-en und der Ärztekammer modifiziert, zusätzlich unter Berücksichtigung weiterer Bilddiagnostik. Das Programm wurde 20 Kollegen vor Ihrer Eingangsprüfung zu Testzwecken zur Verfügung gestellt. Ergebnisse: Die Koppelung von SQL-Datenbank und dem neuen Javaviwer ist den geschlossenen Lehrprogrammen hinsichtlich Erweiterbarkeit, Modifizierung und Anwendungsgebiete weit überlegen. Die plattformübergreifende Programmnutzung ist online und offline möglich und erlaubt so in Verbindung mit verschiedenen Inhalten und Auswertemodulen eine interne Anwendung zur eigenen Qualitätssicherung als auch eine zentrale in der verpflichtenden Qualitätsdiagnostik. Eine Anbindung an bestehende PACS und digitale Mammographieeinrichtungen ist problemlos. Schlussfolgerung: Die Neukonzeption eines DICOM-Javaviewers in Verbindung mit einer SQL-Datenbank eröffnet zahlreiche neue Möglichkeiten in der mammographischen Qualitätssicherung und löst Probleme wie: Durchführung und Auswertung zentraler Prüfungen, automatische Bereitstellung von Mammographiesammlungen, konsiliare Bildanalyse im Netz, Schaffung von Referenzdatenbanken.

\section{V68}

Die stereotaktische Exzisionsbiopsie mit dem ABBI-System Versuch einer abschließenden Beurteilung

\section{*Stefan Krämer ${ }^{1,2}$, Aysen Bilgicyildirim² ${ }^{2}$, Andreas Magener ${ }^{3}$,} Werner Bautz ${ }^{4}$, Matthias Beckmann ${ }^{2}$, R. Schulz-Wendtland ${ }^{4}$ ${ }^{1}$ Abteilung Senologie - Interdisziplinäres Brustzentrum, Klinik der Landeshauptstadt, Düsseldorf-Gerresheim, Deutschland ${ }^{2}$ Frauenklinik, Friedrich-Alexander-Universität, Erlangen-Nürnberg, Deutschland

${ }^{3}$ Institut für Pathologie, Friedrich-Alexander-Universität,

Erlangen-Nürnberg, Deutschland

${ }^{4}$ Gynäkologische Radiologie, Institut für Diagnostische Radiologie, Friedrich-Alexander-Universität, Erlangen-Nürnberg, Deutschland

Seit 1996 haben wir 165 stereotaktische Exzisionsbiopsien mit dem ABBISystem bei klinisch-okkulten, ausschließlich mammographisch darstellbaren Brustläsionen (BI-RADS IV/V, $<1,5 \mathrm{~cm}$ ) durchgeführt. Es wurden ausschließlich Biopsiekanülen mit einem Durchmesser von $2 \mathrm{~cm}$ eingesetzt. In $20(11 \%)$ Fällen war die Durchführung der Methode aufgrund einer unzureichenden Kompressionsdicke der Brust $<2,5 \mathrm{~cm}$, einer thoraxwandnahen oder retromamillären Lokalisation nicht möglich. Eine anschließende offene Exzisionsbiopsie wurde in den folgenden Situationen indiziert: Malignität (CIS oder invasiv), ADH, Diskrepanz in der radiologisch-histopathologischen Korrelation.

Von den 165 Befunden waren 124 benigne und 48 maligne (39 DCIS, 8 IDC, 1 tubuläres Karzinom). Nach ABBI-BIopsie wurden 8 der 9 invasiven Karzinome direkt diagnostiziert, davon 5 mit tumorfreiem Randsaum ohne begleitendes in-situ Karzinom $(5-8 \mathrm{~mm}) .1$ invasives Karzinom wurde erst nach offener Re-Biopsie diagnostiziert, nachdem im ABBIPräparat ein reines DCIS (Herdbefund und Mikrokalk) gefunden wurde. In 4 Fällen wurde auf Wunsch der Patientinnen (>70 Jahre) auf eine anschließende Operation verzichtet, da die Resektionsränder tumorfrei waren $(>3 \mathrm{~mm}$ ). Nach ABBI-Biopsie wurden 36 der 39 DCIS direkt diagnostiziert. 3 DCIS wurden erst nach offener Re-Biopsie diagnostiziert, nachdem in den ABBI-Präparaten eine ADH (Mikrokalk)gefunden 
wurde. Technische Probleme mit der ABBI-Biopsie traten in 5\% der Fälle auf, Komplikationen in 4\%. Die stereotaktische Exzisionsbiopsie ist eine pratikable, sichere und präzise Methode zur Abklärung ausschließ lich mammographisch darstellbarer Herdbefunde der Brust. Im Hinblick auf die Erforschung experimenteller Ablationsverfahren in der Behandlung kleinster, invasiver Mammakarzinome sollte die stereotaktische Exzisionsbiopsie auch unter möglichen operativ-therapeutischen Gesichtspunkten in Diskussion bleiben.

V69

\section{Mammakarzinogenese und Expressionsverlust des Tumorsuppressorgens ARHI}

*Stefan Krämer 1, 2, 3, Robert Luo², Marc Lippmann², Yinhua Yu², Robert Bast Jr. ${ }^{2}$, Matthias Beckmann ${ }^{3}$

${ }^{1}$ Abteilung Senologie - Interdisziplinäres Brustzentrum, Klinik der Landeshauptstadt, Düsseldorf-Gerresheim, Deutschland ${ }^{2}$ Department Experimental Therapeutics, University of Texas, MD Anderson Cancer Center, Houston, USA

${ }^{3}$ Frauenklinik, Friedrich-Alexander-Universität, Erlangen-Nürnberg, Deutschland

Für die Etablierung eines Mehrschrittmodells der Mammakarzinogenese vom normalen duktalen Epithel über das DCIS bis hin zum invasiven Karzinom ist eine Vielzahl relevanter molekularer Alterationen unklar. Ras Homolog I (ARHI) ist ein relevantes Tumorsuppressorgen beim Ovarial- und Mammakarzinom, dessen differentielle Expression in Zellen des normalen duktalen Brustepithels nachweisbar und beim Mammakarzinom durch Allelverlust und epigenetische Mechanismen wie Hypermethylierung des Genpromotors inaktiviert ist (maternales Imprinting). Wir untersuchten die Assoziation des ARHI-Expressionsverlustes als möglichen Faktor der Mehrschrittkarzinogenese der Brust.

Analyse der ARHI-Expression in 94 Praffinpräparaten mit Nachweis von DCIS und normalem Brustepithel in allen Präparaten und Nachweis von Anteilen eines invasiv-duktalen Mammakarzinoms (IDC) in 33 Präparaten. Der ARHI-Expressionsnachweis erfolgte durch in-situ Hybridisierung auf m-RNA Ebene und immunhistochemisch auf Proteinebene.

ARHI-mRNA und Protein konnte in allen normalen Brustepithelien nachgewiesen werden. Im Vergleich zum normalen Brustepithel konnte eine ARHI-Downregulation in 42\% (39 von 94) beim DCIS und in 70\% (23 von 33) beim IDC nachgewiesen werden (davon in 17\% kompletter ARHI-Expressionsverlust). Die Expression von Cyclin D1 und p21WAF1/CIP1 korrelierte invers mit der ARHI-Expression.

Das Ductale Carcinoma in situ (DCIS) der Brust ist mit einem signifikanten Expressionsverlust des Tumorsuppressorgens ARHI assoziiert. Die Progression zum invasiv-duktalen Mammakarzinom (IDC) ist durch einen weiteren ARHI-Expressionsverlust gekennzeichnet.

\section{V70}

\section{Expression von ALCAM/CD166 ist ein unabhängiger Prognosemarker für die Progression des Mammakarzinoms}

*Glen Kristiansen ${ }^{1}$, Mick Burkhardt ${ }^{1}$, Florian Fritzsche ${ }^{1}$, Tserentschunt Gansukh ${ }^{1}$, Stephan Pahl ${ }^{1}$, Carsten Denkert ${ }^{1}$, Wilko Weichert ${ }^{1}$, Joachim Bellach ${ }^{2}$, Hans Guski ${ }^{1}$, Klaus-Jürgen Winzer ${ }^{3}$, Manfred Dietel ${ }^{1}$

${ }^{1}$ Institut für Pathologie, Charité Campus Mitte, Berlin, Deutschland ${ }^{2}$ Tumorzentrum, Charité Campus Mitte, Berlin, Deutschland ${ }^{3}$ Mammazentrum, Charité Campus Mitte, Berlin, Deutschland

Zielsetzung: ALCAM (Activated Leukocyte Cell Adhesion Molecule, CD166), ein CD6-Ligand, ist ein Zelladhäsionsmolekül aus der Immunglobulinfamilie und wird in einer Vielzahl von Geweben exprimiert. Es wurde jüngst als Progressions- bzw. Prognosemarker im Malignen Melanom und im Prostatakarzinom beschrieben. Unser Ziel war die Untersuchung der Expression von ALCAM im Mammakarzinom und die Korrelation der Expression mit klinisch-pathologischen Parametern. Materialien und Methoden: 162 Mammakarzinome (Histologie:132 duktal, 17 lobulär, 13 andere. 103 pT1, 44 pT2, 7 pT3, 8 pT4. 40 G1, 82 G2, 40 G3. 79 $\mathrm{pN} 0,81 \mathrm{pN} 1,2 \mathrm{pNx}$.) wurden immunhistochemisch (Klon NCL-CD166, LSAB-System) auf ihre ALCAM Expression hin untersucht. Zur statisti- schen Auswertung wurden Kreuztabellen, Kaplan-Meier Kurven und eine Cox Regression eingesetzt. Ergebnisse: ALCAM findet sich relativ ubiquitär sowohl in normalen als auch in neoplastischen Epithelien der Mamma mit einem teils membranösen, teils zytoplasmatischen Färbemuster. Eine starke zytoplasmatische ALCAM Expression fand sich in $17 \%$ aller invasiven Mammakarzinome und korrelierte signifikant mit kürzeren progressionsfreien Überlebenszeiten ( 88 vs. 58 Monate, $\mathrm{p}=0,0142$ ), was sich in der multivariaten Analyse bestätigen liess (relatives Risiko 2,$1 ; p=0,026$ ) und somit auch für die Gruppe der nodal negativen Fälle zutraf. Zusammenfassung: ALCAM ist überexprimiert in Neoplasien der Mamma und ist ein neuer, von konventionellen Parametern unabhängiger Prognosemarker des Mammakarzinoms, der möglicherweise zur Risikostratifizierung nodal negativer Mammakarzinome eingesetzt werden kann.

V71

\section{Sentinellymphknoten: Schnellschnittuntersuchung in der Routine?}

*Jörg Linke1, Volker Loy ${ }^{1}$, Michael Wolf ${ }^{2}$

${ }^{1}$ Vivantes Fachbereich Pathologie, Berlin, Deutschland

${ }^{2}$ Zentrum für Brusterkrankungen, Berlin, Deutschland

Zielsetzung: 1. Ist die Schnellschnittuntersuchung von Sentinellymphknoten ausreichend sicher? 2. Detektion aller Mikrometastasen! 3. Wie lassen sich Qualitätsindikatoren dokumentieren? Materialien und Methode: $\mathrm{Ab}$ Juli 2003 untersuchten wir prospektiv die Sentinellymphknoten des Zentrums für Brusterkrankungen parallel im Schnellschnitt und am paraffineingebetteten Gewebe (201 Patienten). Der Lymphknoten wurde in Scheiben $(2 \mathrm{~mm})$ zerlegt, mit einem Lupenmikroskop eine Lamelle für die Schnellschnittuntersuchung ausgewählt und mit Farbe markiert. Die im Schnellschnitt negativen Lymphknoten wurden in Stufen vom $180 \mu$ vollständig aufgearbeitet, einschließlich Immunhistologie. Das Ergebnis für die Schnellschnittuntersuchung wurde in unserem Informationssystem bei der Begutachtung getrennt für die Schnellschnittuntersuchung und die Untersuchung nach Paraffineinbettung dokumentiert.

Ergebnisse:

Negativ richtig ( $\mathrm{pN} 0): 71,14 \%(\mathrm{n}=143)$, darunter 13 Fälle mit isolierten Tumorzellen ( $\mathrm{pN} 0(\mathrm{i}+)$.

Negativ falsch: 7,47\% $(\mathrm{n}=15)$, davon in 8 Fällen nur Mikrometastasen (pN1(mi)).

Positiv richtig: $19,90 \%(\mathrm{n}=40)$, davon in 5 Fällen nur Mikrometastasen (pN1(mi)).

Positiv Verdacht: $1,40 \%(n=3)$, davon in zwei Fällen Mikrometastasen (pN1(mi)).

Zusammenfassung: Entgegen der in der Literatur oft behaupteten Bedeutungslosigkeit einer minutiösen Aufarbeitung der Sentinellymphknoten, sind die publizierten Fakten nach wie vor widersprüchlich. Eine überzeugende Studie fehlt, die bisher veröffentlichten Daten insbesondere über die Zahl der Mikrometastasen und die Rate der im Schnellschnitt falsch negativen Befunde schwankt stark. Da auch bei Mikrometastasen mit therapeutischen Konsequenzen zu rechnen ist, streben wir an $100 \%$ auch der Mikrometastasen zu erfassen. Mit der 6. jetzt gültigen Auflage der TNM-

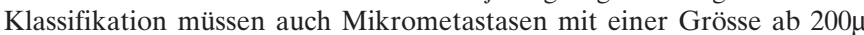
gefunden werden. Gleichzeitig erfolgt eine getrennte Dokumentation der Schnellschnittuntersuchung, die als Qualitätsindikator ein kontinuierliches Monitoring der Arbeitsqualität erlaubt.

\section{V72}

Der Telepathologie-Konsultationsservice des Berufsverbandes deutscher Pathologen

\section{*Thomas Schrader ${ }^{1}$, Peter Hufnagl', Werner Schlake ${ }^{2}$,}

\section{Manfred Dietel ${ }^{1}$}

${ }^{1}$ Institut für Pathologie, Charite Universitätsmedizin Berlin, Berlin, Deutschland

${ }^{2}$ Institut für Pathologie, Gelsenkirchen, Deutschland

Zielsetzung: Im Rahmen der Frühdiagnostik von Mammakarzinomen werden umfassende Maßnahmen zur Qualitätssicherung umgesetzt. Die histopathologischen Befunde müssen von einem zweiten externen Facharzt begutachtet werden. Das Einholen einer zweiten Meinung liegt im 
Fokus der Telepathologie. Auf Grundlage des Telepathology Consultation Centers der UICC (UICC-TPCC) wurde ein Telepathologie-Konsultationsservice für den Berufsverband der deutschen Pathologen entwickelt, um das Einholen der zweiten Meinung im Rahmen des Screening-Programms Mammakarzinom abzubilden. Material und Methoden: Als Kommunikationsmodell liegt ein Client-Server-Modell zu Grunde; der Service ist internetbasiert. Als Webserver wird der Internet Information Server als Datenbank der SQL-Server verwendet. Mittels ASP-Skripte und Javaskript werden die dazustellenden Webseiten und Formulare inklusive der Abfragen und Kontrollmechanismen erzeugt. Ergebnisse: Der Telepathologie-Konultationsservice (TKS-BV) ist ein deutschsprachiger, an den Anwendungsfall angepasster, internetbasierter Telepathologie-Server mit dem einfach zu einem Fall eine zweite Meinung eingeholt werden kann. Alle Pathologen können gleichzeitig als Anfragende oder als Konsilarien in diesem System arbeiten. Alle Vorgänge (Zuweisen eines Falles, Anfragen, Diagnosen usw.) werden vom Server protokolliert. Die Verantwortlichkeit ist durch das Pathologe-Konsilarius-Konzept eindeutig geklärt und nachvollziehbar - im Gegensatz zu Diskussionsgruppen. Derzeit läuft eine Studie zur diagnostischen Validität und zur Praktikabiltät des Systems unter den Routinebedingungen der pathologischen Diagnostik. Zusammenfassung: Der Telepathologie-Konsultationsservice des Berufsverbandes der deutschen Pathologen (TKS-BV) ist ein internetbasiertes Telepathologie-System zum Einholen der geforderten zweiten Meinung speziell für das Screening-Programm zur Früherkennung von Mammatumoren. Durch das Pathologe-Konsilarius-Konzept und eine vollständige Protokollierung aller Vorgänge auf dem Server des TKS-BV sind die Verantwortlichkeiten eindeutig geklärt und transparent nachvollziehbar.In einer Studie wird zurzeit Zuverlässigkeit und Praktikabilität unter Routinebedingungen geprüft.

\section{V73}

\section{Phantomstudie zur Detektion simulierter Läsionen an fünf verschiedenen digitalen und einem konventionellen Mammographiesystem}

${ }^{*} R$. Schulz-Wendtland ${ }^{1}$, K. -P. Hermann ${ }^{2}$, M. Lell ${ }^{1}$, C. Böhner ${ }^{1}$, E. Wenkel ${ }^{1}$, K. Imhoff ${ }^{1}$, A. Schmid ${ }^{1}$, B. Krug ${ }^{3}$, W. Bautz ${ }^{1}$

${ }^{1}$ Universität Erlangen-Nürnberg, Radiologisches Institut (Direktor: Prof. Dr. med. W. Bautz), Erlangen, Deutschland ${ }^{2}$ Georg-August-Universität Göttingen, Bereich Humanmedizin, Abteilung Diagnostische Radiologie

(Direktor: Prof. Dr. med. E. Grabbe), Göttingen, Deutschland ${ }^{3}$ Klinik und Poliklinik für Radiologische Diagnostik, Klinikum der Universität zu Köln (Direktor: Prof. Dr. med. K. Lackner), Köln, Deutschland

Zielsetzung: Experimentelle Phantomstudie zur Detektion simulierter Läsionen an fünf verschiedenen digitalen und einem konventionellen Mammographiesystem. Materialien und Methoden: Es wurden Phantomaufnahmen mit einem konventionellen Film-Folien-System (Mammoma $3000 \mathrm{~N}$ ) (Fa. Siemens) und 5 digitalen Mammographiesystemen (hochauflösendes Speicherfoliensystem (Fa. Fuji/Siemens); a-Si Detektor (Fa. GE Medical Systems); a-Se Detektoren (Fa. Siemens; Fa. Hologic/Lorad) und CCD-Detektor (Fa. Fischer Imaging)) bei gleichen Aufnahmeparametern angefertigt. Testobjekt war das Wisconsin Mammographic Random Phantom, Modell 152 A. Ergebnisse: Es ergaben sich für die konventionelle Film-Folien-Mammographie (84,9\%), die hochauflösende Speicherfolientechnik $(86,7 \%)$ und die digitale Mammographie mit a-Si Detektor $(89,8 \%)$ keine signifikanten Unterschiede bezüglich der Detektion der simulierten Mammaläsionen. Die digitalen a-Se Systeme (97,3\%) und das CCD-System (100\%) erreichten hochsignifikant bessere Detektionsraten ( $\mathrm{p}<0,001)$. Zusammenfassung: Die konventionelle Film-Folien-Mammographie kann durch die digitale Mammographie mit hochauflösenden Speicherfolien und durch die digitale Mammographie mit a-Si-, a-Se-, und CCD-Detektoren ersetzt werden - dies müssen jedoch weitere klinische Studien zeigen.

\section{V74}

Erste Erfahrungen ( $\mathrm{n}=75$ ) mit einem neuen Brust-Vakuumbiopsiesystem (VacuFlash ${ }^{\circledR}$ )

${ }^{*}$ R. Schulz-Wendtland, E. Wenkel, K. Imhoff, M. Bani, A. Magener W. Bautz

Universität, Erlangen-Nürnberg, Deutschland

Zielsetzung: Interventionelle Methoden sind integraler Bestandteil der Mammadiagnostik. Ziel unserer klinischen prospektiven Studie war es, die Möglichkeiten des neuen Brust-Vakuumbiopsiesystems (VakuFlash ${ }^{\circledR}$ ) zu untersuchen. Materialien und Methoden: Von 04/02 bis 09/02 wurde bei 75 Pat. eine sonographisch geführte Brust-Vakuumbiopsie durchgeführt. Die Besonderheit des neuen Systems liegt darin, dass Nadel, mikroprozessgesteuerter Antrieb, Ladeeinheit und der Vakuumbehälter in einem Handgriff integriert sind. Bei allen Pat. wurden die Herdbefunde als sonographisch BI-RADS ${ }^{\circ} 5$ (analog) diagnostiziert (Größe $8 \mathrm{~mm}$ bis $33 \mathrm{~mm}$, median $14 \mathrm{~mm}$ ). Es wurden 5 Biopsien von jedem Herd entnommen. Follow-up mindestens 12 Monate. Ergebnisse: Bei 69 / 75 Pat (92\%) ergab die hist. Diagnose ein Karzinom, bei 6 Pat. (8\%) handelte es sich um Fibroadenome $(\mathrm{n}=2)$, Mastopathie $(\mathrm{n}=2)$, Fibrose $(\mathrm{n}=2)$ - dieses wurde op.-hist. bestätigt. Die Ergebnisse entsprechen den Vorgaben des ACR. Zusammenfassung: VakuFlash ${ }^{\circledR}$ ist ein technisch ausgereiftes Instrument, welches für die sonographisch geführte Biopsie bei Mammaläsionen der Klassifikation BI-RADS 4 / 5 (analog) gut geeignet ist.

\section{V75}

\section{Identifizierung neuer Progressions-abhängiger Markergene beim Mammakarzinom mittels Lasermikrodissektion und Mikroarrayanalyse}

${ }^{*}$ Christina Schütz ${ }^{1}$, Hans Neubauer ${ }^{1}$, Michael Bonin ${ }^{2}$, Susan Clare ${ }^{3}$ Karl Sotlar ${ }^{4}$, Olaf Rieß², Diethelm Wallwiener ${ }^{1}$, Raffael Kurek ${ }^{1}$ ${ }^{1}$ Universitäts-Frauenklinik, Tübingen, Deutschland

${ }^{2}$ Medizinische Genetik, Tübingen, Deutschland IIndiana University School of Medicine, Indianapolis, USA ${ }^{4}$ Institut für Pathologie, Tübingen, Deutschland

Molekulargenetisch betrachtet ist das Mammakarzinom eine komplexe heterogene Erkrankung, die durch größtenteils noch ungeklärte Interaktionen genetischer und nicht genetischer Faktoren entsteht. Die Verbesserung des aktuellen therapeutischen Standards durch Individualisierung der bislang nur empirisch festgelegten Therapie ist dringend notwendig. Ziel des Projektes ist die Analyse progressionsstufentypischer Expressionsprofile des duktalinvasiven Mammakarzinoms, um neue, klinisch relevanter Phänotypen und Genpathways zu identifizieren. Die Kombination von Mikroarrayanalyse und Lasermikrodissektion (LCM) ermöglichte die Untersuchung homogenen Ausgangsmaterials, von je DCIS und IDC-Komponente aus fünf Tumoren. Die Isolierung der Gesamt-RNA (Qiagen) erfolgte nach Dissektion von 20 000 Zellen (7000 Laserpulsen, $30 \mu \mathrm{m}$, Arctutus PixCell IIe) aus Hämatoxylin/Eosin-gefärbten Tumorschnitten. Ein Protokoll für die lineare RNA-Amplifikation über zwei Runden (50-100 ng Ausgangsmaterial) wurde erfolgreich etabliert und angewendet. Qualitätskontrollen vor und nach der LCM, während und nach der Amplifikation erfolgten mit Kapillargelelektrophorese (Agilent, Bioanalyzer). Ergebnisse der Test-Arrays (Affymetrix) zeigten die gute Qualität der Proben mit niedrigen 3'/5'-Verhältnissen (HUMGAPDH/ M33197: 1,5 -2,5; HSAC07/X00351: 9-12). Datenanalysen nach Hybridisierung auf Affymetrix U133A Arrays wurden mit Hilfe des Data Mining Tools (Affymetrix) und Genesis (A. Sturn, Graz) durchgeführt. Kandidatengene wurden definiert als signifikant (ANOVA) differentiell exprimierte Gene zwischen DCIS und IDC-Komponente, die in allen fünf Tumoren gleichermaßen reguliert waren. Quantitative RT-PCR-Analysen (Lightcycler, ROCHE) bestätigten die Mikroarray-Resultate für bekannte Brustkrebsgene (z. B. Versican, PLAU) und neue Kandidatengene. Diese sollen im Weiteren mit Tissue-Arrays an einem großen Probenkollektiv validiert werden. Zusammenfassend wurde durch die Untersuchung hochreinen Ausgangsmaterials neue molekulare Markergene identifiziert, die den Übergang vom DCIS zum IDC charakterisieren, und die somit neue potentielle Prognose-/Prädiktionsfaktoren bzw. «Drug-Targets» repräsentieren. 
V76

Ist ein präoperatives Lokalstaging mittels MR-Mammografie (MRM) bei histologisch gesichertem Mamma-Karzinom angesichts der resultierenden falsch positiven Biopsien zu rechtfertigen?

${ }^{*}$ Katja C. Siegmann ${ }^{1}$, Philip Neugebauer ${ }^{1}$, Tanja Xydeas ${ }^{1}$, Stephan Miller ${ }^{1}$, Ute Krainick-Strobel ${ }^{2}$, Claus D. Claussen ${ }^{1}$

${ }^{1}$ Abteilung Radiologische Diagnostik, Universitätsklinikum Tübingen, Tübingen, Deutschland

${ }^{2}$ Universitätsfrauenklinik Tübingen, Tübingen, Deutschland

Zielsetzung: Die MR-Mammografie ist eine sensitive Methode zum Nachweis multifokaler/kontralateraler Herde bei Frauen mit Mamma-Karzinom. Aufgrund der moderaten Spezifität des Verfahrens werden auch benigne Herde in Form suspekt enhancender Läsionen detektiert. Das Zie der Studie war es die Rate der MR-bedingten Therapieänderungen und die daraus resultierenden Vor- und Nachteile für die Patientinnen zu evaluieren. Materialien and Methoden: Wir untersuchten konsekutiv $128 \mathrm{~Pa}-$ tientinnen mit histologisch gesichertem Mamma-Karzinom. Die Anzahl der aufgrund der MR-Mammografie veranlassten Therapieänderungen wurde gezählt. Alle mammografisch und sonografisch okkulten Befunde wurden histologisch gesichert. Alle zusätzlich entdeckten malignen Läsionen und eingesparten Operationen aufgrund negativer MR-Befunde wurden als vorteilhaft für die Patientinnen eingestuft. Ergebnisse: Die Ergebnisse der präoperativen MR-Mammografie haben das therapeutische Vorgehen in 47 von 128 Fällen verändert (36,7\%): Bei 17 Patientinnen wurde anstelle einer brusterhaltenden Operation eine Mastektomie durchgeführt, 6 Frauen erhielten eine größere Exzision als vorgesehen, 21 zusätzlich detektierte Läsionen wurden MR-gestützt markiert und exzidiert, in zwei Fällen wurde eine zusätzliche perkutane Biopsie durchgeführt und bei einer Patientin wurde nach unnauffälligem MRM-Befund auf eine geplante Exzisionsbiopsie verzichtet. Die histologisch gesicherten Läsionen waren in $67,4 \%$ (31/46) maligne. Somit waren durch Hinzuziehung einer Patientin, die durch negativen MR-Befund eine geplante Exzision vermeiden konnte, die MRM-bedingten Therapieänderungen bei $68,1 \%(32 / 47)$ vorteilhaft für die Patientinnen. Schlussfolgerung: Bei Mamma-Karzinom ist ein präoperatives Lokalstaging mittels MRM vorteilhaft für die Patientinnen, da okkulte Zweitkarzinome detektiert werden und die Ausdehnung des Primarius besser dargestellt wird. Vor diesem Hintergrund erscheint eine Rate von 11,7\% (15/128) zusätzlich durchgeführten benignen Biopsien aufgrund suspekter MRM-Befunde vertretbar.

\section{V77}

\section{Familiärer Brustkrebs: Erste Ergebnisse zur Früherkennung des Mammakarzinoms an einem humangenetisch definierten Risikokollektiv im Zentrum Münster}

Bernd Tombach', Stefanie Weigel ${ }^{1},{ }^{*}$ Eva Maria Fallenberg ${ }^{1}$, Stefan Krämer ${ }^{1}$, Rainald Bachmann ${ }^{1}$, Dennis Nordhoff', Brigitte Welling ${ }^{2}$, Jürgen Horst ${ }^{2}$, Walter Heindel ${ }^{1}$

${ }^{1}$ Institut für Klinische Radiologie, Münster, Deutschland ${ }^{2}$ Institut für Humangenetik, Universitätsklinikum, Münster, Deutschland

Zielsetzung: Wie hoch ist die detektierte Mammakarzinomrate in einem humangenetisch definierten Risikokollektiv in einer 1. Screeningrunde, die Mammographie, Ultraschall und MR-Mammographie (MRM) umfaßt? Materialien und Methoden: Als Teil einer von der Deutschen Krebshilfe unterstützten Multicenterstudie wurden im Zentrum Münster von 12/02-03/04 112 Frauen (30-71 Jahre) eingeschlossen, die nach den Chang-Claude-Tabellen ein medianes Risiko von $35 \%$ zugewiesen bekamen. In diesem Kollektiv lagen bei $\mathrm{n}=11$ Frauen eine positive BRCA-1 Testung, bei $n=3$ eine positive BRCA-2-Testung und bei $n=14$ nachgewiesene BRCA-Varianten (UV) vor. Dem jährlichen Intervall der Mammographie angepaßt, wurden am gleichen Tag eine Mammographie, eine Ultraschalluntersuchung $(7,5 \mathrm{MHz})$ und eine MRM $(1,5 \mathrm{~T})$ zunächst separat durchgeführt und dann synoptisch befundet. Ergebnisse: Es wurden 5 T1-Karzinome bei 3 Frauen im Alter von 39 bis 63 Jahren richtig positiv diagnostiziert (Risiko: 20\% (BRCA-UV); 16\% (keine Testung); 70\% (BRCA-2)). In der MRM wurden 5/5 Karzinome prospektiv detektiert, mit dem Ultraschall 3/5 und mit der Mammographie 1/5. Zusätzlich wur- den 2 falsch positive Befunde (Fibroadenom, Skleradenose) aufgrund der MRM- bzw. der Mammographiebildgebung histologisch gesichert neben 1 richtig negativen Befund (größenprogredientes Fibroadenom). Zusammenfassung: Die detektierte Karzinomrate ohne mögliche ausstehende falsch negativ bestätigte Befunde aus der künftigen 2. Screeningrunde übersteigt in dem humangenetisch rekrutierten Kollektiv die zu erwartende sporadische Rate. Die MRM ermöglicht im Vergleich zur Mammographie und zum Ultraschall die höchste Detektionsrate.

V78

\section{Evaluation von Qualitätsindikatoren des Mammographie-} Screenings Weser-Ems (MSWE) durch Abgleich mit dem Epidemiologischen Krebsregister Niedersachsen (EKN)

*Iris Urbschat ${ }^{1}$, Joachim Kieschke ${ }^{1}$, Ursula Schlanstedt-Jahn" Sandra von Gehlen ${ }^{2}$, Andreas Thiel ${ }^{3}$, Peter Jensch ${ }^{2,3}$ ${ }^{1}$ Epidemiologisches Krebsregister Niedersachsen, Oldenburg-Hannover, Deutschland ${ }^{2}$ OFFIS, Oldenburg, Deutschland

${ }^{3}$ Carl von Ossietzky Universität, Oldenburg, Deutschland

Zielsetzung: Ziel des bundesweiten Mammographie-Screenings ist die Senkung der Brustkrebsmortalität. Im Vorfeld zur bundesweiten Einführung fand in Weser-Ems, Bremen und Wiesbaden die Erprobung des Mammographie-Screenings statt. Als frühe Prediktoren für die Mortalitätsreduzierung gelten Intervallkarzinomrate, Tumorstadienverteilung, Anteil von invasiven Tumoren und regionären Lymphknotenmetastasen, die mit Nicht-Teilnehmerinnen zu vergleichen sind. Diese Qualitätsparameter sind über einen Abgleich mit bevölkerungsbezogenen Krebsregistern zu evaluieren. Methodik: Bezugsbevölkerung sind zirka 22000 50-69jährige Frauen, die seit Mai 2002 zum Mammographie-Screening eingeladen werden. Der Abgleich der Teilnehmerinnen-Daten mit dem Krebsregister erfolgt über ein probabilistisches Record-Linkage-Verfahren pseudonymisiert über EKN-Kontrollnummern. Die Vollzähligkeit des Registers liegt für Brustkrebs bei über 90\%. Ergebnisse: zirka 13000 Frauen haben bisher am Mammographie-Screening teilgenommen (Teilnahmerate $65 \%$ ); für $5,9 \%$ der Teilnehmerinnen erfolgte eine Weiterbetreuung im Assessment. Bis Juli 2003 wurden bei 70 Frauen invasive und nicht-invasive Mammakarzinome diagnostiziert. Im ersten Abgleich mit dem EKN wurden zwei Intervallkarzinome ermittelt, für die eine nachgehende Qualitätssicherung im MSWE erfolgen soll (Klassifikation in true interval, radiologically occult, minimal signs, false negative, unclassifiable). Erste Analysen der Tumorstadien zeigen erwartungsgemäß eine $\mathrm{Zu}-$ nahme des Anteils von in-situ-Tumoren sowie Tumoren $\leq 10 \mathrm{~mm}$ von $17,9 \%$ (2001) auf 30,6\% (2002). Die Inzidenzraten von größeren Tumoren (TNM-Stage II-IV) werden dargestellt. Zusammenfassung: Erste Effekte des Mammographie-Screenings sind durch Abgleich mit bevölkerungsbezogenen Krebsregistern frühzeitig evaluierbar. Mit der Nutzung des EKN-Kontrollnummersystems ist der Abgleich unter Einhaltung datenschutzrechtlicher Bestimmungen durchführbar. Problematisch ist, dass aufgrund landesrechtlicher Bestimmungen für die Rückmeldung von Intervallkarzinomen an das MSWE nachträglich eine Patienten-Einwilligung einzuholen ist. Die Umsetzbarkeit der Krebsfrüherkennungsrichtlinen in den Krebsregistern wird diskutiert.

\section{V79}

Verbesserte morphologische Beschreibung KM-aufnehmender Läsionen in der dynamischen MR-Mammographie durch die Kombination von hoch und niedrig aufgelösten Sequenzen in zwei räumlichen Ebenen

*Toni W. Vomweg, Andrea Teifke, R. Peter Kunz, Christian Hintze, Alexander Hlawatsch, Annett Kern, Karl F. Kreitner, Manfred Thelen Klinik und Poliklinik für Radiologie,

Klinikum der Johannes Gutenberg-Universität, Mainz, Deutschland

Ziele: Erstellung einer Kombination von niedrig- und hoch-aufgelösten T1-gewichteten 3D Gradient Echo Sequenzen (mit kurzen und langen Messzeiten) in zwei Projektionen zur Verbesserung der morphologischen Analyse ohne Kompromittierung der Auswertung der KM-Dynamik. Material und Methoden: 3D-FLASH Sequenzen mit unterschiedlicher 
Matrix $(256 \times 128$ Pixel und $512 \times 256$ Pixel, 72 Schichten, Schichtdicke $1,7 \mathrm{~mm}, \mathrm{TR} 8,8 \mathrm{~ms}$, TE $4,5 \mathrm{~ms}, 25^{\circ}$ Flipwinkel) wurden in einer speziellen Reihenfolge zur einem Protokoll kombiniert, so dass hoch und niedrig aufgelöste Subtraktionsbilder erzeugt werden können. Drei Untersucher beurteilten mit Hilfe einer Punkteskala morphologische Eigenschaften von insgesamt 36 histologisch gesicherten, KM-aufnehmenden Läsionen in zufälliger Reihenfolge anhand niedrig oder hoch aufgelöster Subtrak tionsbilder. Ergebnisse: Die Einschätzung der Dignität der Läsionen anhand des morphologischen Scores verbesserte sich bei allen drei Untersuchern. (Untersucher A: 8 richtige Korrekturen, 3 falsche Korrekturen, B 12/5, C: 16/4). Insbesondere die Detektion von Malignitätskriterien (Spikulierung, irreguläre Außenkontur) wurde durch die hoch-aufgelösten Subtraktionsbilder in koronarer und transversaler Orientierung erleichtert. Die Variabilität der Scores der einzelnen Untersucher wurde deutlich verringert, der mittlere kappa-Wert erhöhte sich signifikant $(\mathrm{p}<0,05)$ von 0,185 auf 0,422 . Zusammenfassung: Die Kombination von niedrig und hoch-aufgelösten Sequenzen zur dynamischen MR-Mammographie führt bei geringfügiger Verlängerung der Messzeit zu einer signifikanten Verbesserung der Analyse morphlogischer Eigenschaften.

\section{V80}

\section{Brustkrebsfrüherkennung in Deutschland - Zwischenergebnisse nach über 95000 Einladungen zum Mammographie-Screening nach den Europäischen Leitlinien}

*Lawrence von Karsa ${ }^{1}$, Hans Junkermann ${ }^{2}$, Margrit Reichel3, Gerold Hecht ${ }^{4}$, Werner Böcker ${ }^{5}$, Nikolaus Becker ${ }^{6}$, Ute Wülfing ${ }^{7}$ ${ }^{1}$ Kooperationsgemeinschaft Mammographie Köln, Köln, Deutschland ${ }^{2}$ Mammographie Screening Bremen, Bremen, Deutschland ${ }^{3}$ Mammographie Screening Wiesbaden, Wiesbaden, Deutschland ${ }^{4}$ Mammographie Screening, Weser-Ems, Deutschland

${ }^{5}$ Gerhard Domagk-Institut für Pathologie der Universität Münster, Münster, Deutschland

${ }^{6}$ DKFZ Heidelberg Abt. für klinische Epidemiologie, Heidelberg, Deutschland

Zielsetzung: Ziel der Brustkrebsfrüherkennung ist die Senkung der Brustkrebssterblichkeit. Die einzige Früherkennungsmethode, die nachweislich die Brustkrebssterblichkeit senken kann, ist das Mammographie-Screening mit Einladungssystem. Vor Aufnahme des Mammographie-Screenings in das Gesetzliche KFU-Programm wurden Modellprojekte nach den Europäischen Leitlinien durchgeführt. An den in den EU-Leitlinien niedergelegten Anforderungen an die Prozess-, Struktur- und Ergebnisqualität werden nun die deutschen Modellprojekte gemessen. Material und Methode: Bis Juni 2003 wurden 95617 Frauen zum bevölkerungsbezogenen qualitätsgesicherten Mammographie-Screening schriftlich eingeladen. Bis Juli 2003 nahmen 53223 Frauen am Screening teil. Die erste Runde wird voraussichtlich im Herbst 2004 abgeschlossen. Die medizinischen Daten der einzelnen diagnostischen Schritte (Screeningkette) werden auf der Basis der Europäischen Leitlinien analysiert und bewertet. Ergebnisse und Zusammenfassung: Einbestellung zur Abklärung: 5,9\%, Punktionsbiopsie: $1,8 \%$, primär empfohlene offene Biopsie: $<0,1 \%$, Karzinomentdeckungsrate: 9,3/1,000. Schon ausgewählte Werte zeigen: Die Umsetzung der in den Europäischen Leitlinien festgelegten Vorgaben zu Prozess- und Strukturqualität ermöglichte die Erreichung der Zielwerte ausgewählter Indikatoren für die Aufbauphase für ein erfolgreiches Früherkennungsprogramm (Ausnahme: die bisherige Teilnahmerate von zirka $50 \%$ ). Die bisherigen vorläufigen Ergebnisse lassen erwarten, dass be Weiterführung des qualitätsgesicherten Mammographie-Screenings langfristig eine deutliche Senkung der Brustkrebssterblichkeit eintreten wird.
V81

\section{Der Splicingfaktor Tra2-beta wird in primären} Mammakarzinomen spezifisch überexprimiert

Dirk Watermann, Markus Jäger, Martha Földi, Gerald Gitsch, *Elmar Stickeler

Universitätsfrauenklinik, Freiburg, Deutschland

Zielsetzung: Die beiden humanen Tra2 Proteine hTra2-alpha und hTra2beta 1, sind Mitglieder der Familie der Serin-Argenin-reichen (SR) Splicingfaktoren und regulieren alternatives Splicing von Exonen mit C/Areichen Exonenhancer Sequenzen. Das CD44 Gen, das für ein extrazelluläres Matrixprotein kodiert, besitzt 10 alternative Exone, die extensiv alternativ gesplict werden. Die variablen Exone v4-v6 besitzen C/A-reiche Enhancer Sequenzen und zeigen ein induziertes Splicing in Mammakarzinomen. Ihnen wird eine funktionelle Bedeutung bei der Progression und Metastasierung von Mammakarzinomen zugemessen. Material und Methoden: In dieser Studie untersuchten wir die das alternative CD44 Splicing und die Expression von hTRa2-alpha und hTra2-beta1 Proteinen in Tumorproben von Mammakarzinomen und korrespondierendem Normalgewebe von Patientinnen mit histologisch nachgewiesenem Mammakarzinom durch Western Blot und RT-PCR Analyse. Ergebnisse: Unsere Analysen ergaben, dass eine Induktion des alternativen CD44 Splicings von einer einer ausgeprägten, spezifischen Erhöhung der hTra2-beta1 Expression in Karzinomen im Vergleich zu Normalgewebe kommt. Das hTRa2-alpha dagegen, war sowohl in Normalgewebe als auch im Tumor nur in geringer konstanter Konzentration nachweisbar. Zusammenfassung: Unsere Experimente lassen in Zusammenschau mit unseren bekannten funktionellen Daten darauf schließen, dass hTra2 in die spezifische Regulation des alternativen CD44 Splicings involviert ist. Diese funktionelle Bedeutung, zusammen mit der Beobachtung der Überexpression von hTra2-beta1, könnte für das alternative Splicing derjenigen CD44 Isoformen von großer Bedeutung sein, die mit Tumorprogression und Metastasierung des Mammakarzinoms assoziiert werden.

\section{V82}

\section{Ein diagnostischer Index zur sonographischen} Dignitätseinschätzung von axillären Lymphknoten beim Mammakarzinom

Dirk Watermann ${ }^{1}$, Kirsten Hörig ${ }^{1}$, Marzenna Orlowska-Volk², Annette Hasenburg ${ }^{1}$, Willi Sauerbre ${ }^{3},{ }^{*}$ Elmar Stickeler $^{1}$ ${ }^{1}$ Universitätsfrauenklinik, Freiburg, Deutschland

2Institut für Pathologie am Universitätsklinikum, Freiburg, Deutschland

${ }^{3}$ Institut für Biometrie und Medizinische Informatik (IMBI), Freiburg, Deutschland

Zielsetzung: Die Dignitätseinschätzung der axillären Lymphknoten bei Patientinnen mit Mammakarzinom gewinnt auf Grund der Sentinel-Biopsie und der neoadjuvanten Chemotherapieprotokolle an Bedeutung. Zur Verbesserung der Aussagekraft der Sonographie kann ein diagnostischer Index beitragen. Methoden: Bei 59 Mammakarzinom-Patientinnen wurden 452 Lymphknoten einzeln präpariert, unter Verwendung von 3D Ultraschall im Wasserbad untersucht und anschließend histologisch aufgearbeitet. Folgende Parameter wurden bestimmt: Metrik (max. Durchmesser und Volumen des Lymphknotens, Maximaler Durchmesser und Volumen des Hilus), Form (oval, rund, irregulär) sowie die Echogenität von Hilus und Rinde (jeweils: homogen, inhomogen, inhomogen mit stark echoarmem Fokus, nicht darstellbar). Die Daten wurden mit einem multivariaten Modell analysiert. Hierbei wurde neben der Abhängigkeit der erhobenen Parameter von der Dignität ebenfalls die Abhängigkeit der Parameter von der einzelnen Patientin berücksichtigt. Anhand der Daten wurde ein diagnostischer Index abgeleitet. Ergebnisse: Von 452 Lymphknoten waren 75 metastatisch befallen, 377 waren tumorfrei. Keiner der metrischen Parameter war in der multivariaten Analyse signifikant mit der Dignität der Lymphknoten assoziiert. Ein signifikanter Zusammenhang zum metastatischen Befall ergab sich bei einer inhomogenen Rinde $(p=0,0021)$, insbesondere wenn diese zusätzlich einen echoarmen Fokus zeigte $(p<0,0001)$. Auch bei einem inhomogenem Hilus $(p<0,0001)$ mit echoarmen Fokus $(p=0,02)$ ergab sich ein signifikanter Zusammenhang. Eine runde $(p=0,0003)$ oder irreguläre $(p=0,004)$ Form war ebenfalls 
signifiknat mit einem metastatischen Befall assoziiert. Aus den Daten wurde ein auf 4 Parametern basierender diagnostischer Index abgeleitet, mit dem zirka 90\% der Lymphknoten richtig klassifiziert werden können. Zusammenfassung: Anhand des verwendeten in-vitro Models wurde ein praktikabler diagnostischer Index abgeleitet, dessen Evaluation in-vivo im Weiteren erfolgen sollte.

\section{V83}

\section{Aussagekraft der 3D-Sonographie zur Beurteilung solider Herdbefunde der Mamma}

Dirk Watermann ${ }^{1}$, Aida Hanjalic-Beck ${ }^{1},{ }^{*}$ Martha Földi ${ }^{1}$, Annette Hasenburg ${ }^{1}$, Heinrich Prömpeler ${ }^{1}$, Bettina Randelzhofer ${ }^{1}$, Kirsten Hörig ${ }^{1}$, Anne Lüghausen ${ }^{1}$, Willi Sauerbrei ${ }^{2}$, Elmar Stickeler ${ }^{1}$ ${ }^{1}$ Universitätsfrauenklinik, Freiburg, Deutschland

${ }^{2}$ Institut für Biometrie und Medizinische Informatik (IMBI),

Freiburg, Deutschland

Zielsetzung: Die diagnostische Aussagekraft der 3D-Sonographie soll durch einen Vergleich mit der konventionellen Sonographie als Standardverfahren überprüft werden. Methoden: In einer prospektiven Untersuchung wurden 100 bioptisch gesicherte, solide Mammabefunde (53 Karzinome, 47 benigne Befunde) von neun unabhängigen Untersuchern beurteilt. Die Befunde wurden anhand eines digitaler 3D-Volumenblocks und digital gespeicherter B-Bilder unabhängig voneinander bewertet. Klinische Daten waren den Untersuchern nicht bekannt. Auf einem Dokumentationsbogens wurden Ultraschallkriterien (zehn für B-Bilder, vierzehn für 3D-Volumenblöcke) beurteilt. Die Dignität wurde auf einer vierstufigen Skala eingeschätzt, in dieser vereinfachenden Analyse wird nur eine zweistufige Einteilung betrachtet. Ergebnisse: Ohne Berücksichtigung der Neunfachbeurteilung jedes Befundes lagen insgesamt 900 Datensätze zur Beurteilung vor. Anhand der B-Bilder wurde die Dignität 705 mal (78,3\%) richtig eingeschätzt, mit der 3D-Sonographie wurde 701 mal (77,9\%) richtig beurteilt. Die falsch negativ Rate (Beurteilung benig ne bei gesichertem Malignom) betrug bei der B-Bild Sonographie 13,6\%, bei der 3D-Sonographie $11,9 \%$, die falsch positiv Rate betrug $8,1 \%$ bzw. $10,2 \%$. Eine konkordante Beurteilung der Befunde mit beiden Methoden lag 702 mal $(78,0 \%)$ vor. Das Retraktionsphänomen in der koronaren Ebene der 3D-Volumenblöcke wurde bei 48,2\% der Beobachtungen an malignen Befunden beschrieben, im Vergleich zu 7,1\% bei den benignen Befunden. Zusammenfassung: Basierend auf einer vereinfachenden Ana- lyse sind die 3D-Sonographie und die konventionellen Sonographie bezüglich ihrer diagnostische Aussagekraft vergleichbar, allerdings liegen häufig diskordante Ergebnisse vor. Anhand detaillierter Analyse müssen die Gründe untersucht werden. Weiterhin ist der Einfluß des Untersuchers und die Bedeutung der einzelnen Ultraschallkriterien zu prüfen. Kriterien zur Verringerung der Fehlerrate sind zu untersuchen.

\section{V84}

Nuklearmedizinische Diagnostik vor und nach autologer Lymphgefäß-Transplantation: Qualitative versus quantitative Lymphszintigraphie

${ }^{*}$ Mayo Weiss ${ }^{1}$, Rüdiger G. H. Baumeister ${ }^{2}$, Klaus Hahn ${ }^{1}$

${ }^{1}$ Klinik und Poliklinik für Nuklearmedizin, München, Deutschland ${ }^{2}$ Chirurgische Klinik, Abt. für Mikro-, Hand- u. Rekonstruktionschirurgie, Ludwig-Maximilians-Universität, München, Deutschland

Zielsetzung: Die autologe Lymphgefäß-Transplantation führt beim peripheren Lymphödem des Armes zu einer signifikanten Verbesserung der Lymphabflussverhältnisse. Die vorliegende Studie untersucht, ob die visuelle Beurteilung der Lympszintigraphie mittels Transport-Index geeignet ist, die persistierende Funktion autologer Lymphgefäß-Transplantate und damit den Erfolg dieser Operationsmethode ausreichend $\mathrm{zu}$ dokumentieren oder ob zusätzliche quantifizierende Auswerteverfahren mittels region-of-interest-Technik zu einer Steigerung der klinischen Aussagekraft führen. Materialen und Methoden: Es wurden sowohl prä- als auch posttherapeutisch lymphszintigraphische Untersuchungen des Armes an einer Großfeld-Gammakamera (low-energy-high-resolutionKollimator) durchgeführt. Dazu erfolgte die Applikation von $70 \mathrm{MBq}$ Tc99m-markierter Kolloide (humanes Serumalbumin, Partikelgröße $80 \mathrm{~nm}$ ) subcutan in den ersten Interdigitalraum der betroffenen Extremität. Nach Stimulation des Lymphabflusses durch aktive Muskelanstrengung erfolgten dynamische Aufnahmen bis zu maximal 6h p. i. Zur Auswertung erfolgte neben der visuellen Beurteilung sowohl eine Quantifizierung mittels nummerischem Transportindex als auch mittels region-of-interestTechnik. Ergebnisse: Die visuelle Auswertung zeigt eine gute Korrelation zwischen lymphszintigraphischem und klinischem Befund; die zusätzlich durchgeführte Quantifizierung mittels region-of-interest-Technik führt unter standardisierten Untersuchungsbedingungen zu einer Objektivierung der Befundung. 


\section{Poster: Operative Therapien}

P1

\section{Trichterbrust - Langzeitresultate mit custom-made} Silikonimplantaten

*Bianca Baican, Oliver Scheufler, Gabriel Marey, Klaus Exner Klinik für Plastische Chirurgie, Wiederherstellungsund Handchirurgie, Markus-Krankenhaus, Frankfurt, Deutschland

Bei der Trichterbrustdeformität ohne pulmonale oder kardiale Funktionsstörungen ist der Defektausgleich mit Silikonimplantaten eine Standardmethode. Langzeitstudien sind bisher nicht publiziert worden.

Von 1977 bis 2002 wurden im Markus-Krankenhaus 48 Implantate be Patienten im Alter zwischen 14 und 52 Jahren eingebracht. Das Geschlechtsverhältnis weiblich zu männlich war 1:1,9. Die Nachuntersuchungen bezogen sich auf subjektive Beschwerden, Infekte und Korrektureingriffe und erfolgten mit einer Fragebogenaktion.

Von den insgesamt 48 Patienten waren 10 waren unbekannt verzogen. 5 Patienten waren an anderen Erkrankungen verstorben. Von 33 erreichbaren Patienten haben 22 den Fragebogen vollständig beantwortet. Zusätzlich wurden 14 Patienten klinisch nachuntersucht.

Subjektive Beschwerden wurden von 2 Patienten geäußert. Hierbei handelte es sich um tastbare Implantatränder und Dislokationen. Rezidivierende Serome traten bei 2 Patienten auf, so dass in einem Fall das Implantat entfernt wurde jedoch ohne Nachweis pathogener Keime. Insgesamt waren 20 Patienten mit dem Ergebnis zufrieden. Bei den weiblichen $\mathrm{Pa}$ tienten mit begleitender Mammaasymmetrie war der vollständige Ausgleich des Konturdefektes auch mit zusätzlichen Brustimplantaten nicht immer möglich.

Die niedrige Komplikationsrate im Vergleich zu operativen Methoden mit Osteotomien und die guten Langzeitresultate rechtfertigen den alloplastischen Konturausgleich bei der Trichterbrust.

\section{P2}

\section{Ist der Latissimus-dorsi-Myokutanlappen für eine ausschließliche Eigengewebsrekonstruktion bei kleiner Brust geeignet?}

*Lelia Bauer, Patricia Faas-Fehervary, Frank Melchert Universitätsfrauenklinik Manheim, Mannheim, Deutschland

Die autologe Brustrekonstruktion mit Latissimus-dorsi (Lado)-Myokutanlappen nach Ablatio mammae wegen Mammakarzinom stellt eine gängige Behandlungsoption dar. Material und Methode: Wir präsentieren eine Gruppe von fünf Mammakarzinom-Patientinnen mit kleiner Brust, die in einem Primär- oder Sekundäreingriff mit Lado-Myokutanlappen versorgt wurden. Das direkt postoperativ erzielte Ergebis war gut, nach 6-12 Monaten stellten sich die Patientinnen mit Wunsch nach Augmentation der operierten Seite vor. Nach Implantateinlage wurde in allen Fällen ein zufriedenstellendes kosmetisches Ergebnis erzielt. Ergebnis: Der gestielte Lado-Myokutanlappen ist eine sehr sichere Operationsmethode unter Verwendung von Eigengewebe. Das geringe Gewebsvolumen, welches durch Entnahme am Rücken erzielt werden kann, ermöglicht die Deckung großer Volumendefekte im Rahmen der brusterhaltenden Therapie. Zur ausschließlichen Eigenrekonstruktion der Brust reicht es jedoch in den meisten Fällen nicht aus. Im weiteren postoperativen Verlauf kommt es zur Muskelatrophie. Daher ist in der Regel auch bei kleiner Brust das Einlegen eines Implantats erforderlich, um ein schönes kosmetisches Ergebnis zu erzielen.
P3

Der multipedikuläre freie DIEP- Lappen zur Rekonstruktion der palliativen Thoraxwandresektion beim Mamma- Carcinom

F. Busse, C. Kim, G. Maiwald, W. Richter, A. Peek

Klinik für Plastische und Handchirurgie, Brustzentrum,

Behandlungszentrum Vogtareuth, Vogtareuth, Deutschland

Einleitung: Thoraxwanddefekte beim Mamma-Carcinom können nach Resektion von primär exulzerierten Carcinomen, von Lokalrezidiven oder von radiogenen Schäden auftreten. Plastisch- chirurgische Deckungsverfahren können dabei unter kurativer oder unter palliativer Zielsetzung zur Verbesserung der Lebensqualität zur Anwendung kommen. Material und Methode: In der Zeit von Januar 2001 bis Juni 2004 wurden 8 Patientinnen (35-68 Jahre, Durchschnittsalter 61 Jahre) mit einem Thoraxwanddefekt operiert. In 4 Fällen handelte es sich dabei um exulzerierte primäre Mammarcarcinome, in 2 Fällen um ein exulzeriertes Rezidiv eines Mammacarcinoms, eine Patientin hatte einen Z.n. MRM, in 1 Fall fand sich ein 9,3kg schweres, 40x30x25 cm großes Liposarkom der rechten Thoraxwand. Eine Palliativsituation mit Fernmetastasen fand sich bei 7 Patientinnen. Präoperativ erfolgte bei allen Patientinnen eine Staginguntersuchung sowie eine CT- bzw. MRT- Untersuchung des Thorax zur Klärung der Infiltrationstiefe. Nach Resektion des Tumors erfolgte die Defektdeckung in allen Fällen durch eine multipedikuläre Perforator- Lappenplastik vom Unterbauch (Deep inferior epigastric perforator- flap), wobei zur Sicherung der Lappendurchblutung ein Pedikel an die A. und V. thoracica interna und ein zweiter an die axillären Gefäße (A. und V. thoracica lateralis oder thoracodorsalis) angeschlossen wurde. Ergebnisse: Bei 7 von 8 Patientinnen konnte eine ROResektion des thorakalen Befundes erzielt werden. In 5 von 8 Fällen heilten die Perforatorlappen primär ein, in 2 Fällen mußten wegen Perfusionsstörungen eine Lappenrevision durchgeführt werden, wobei durch eine Lappenteilresektion ein sekundärer vollständiger Wundverschluß erzielt werden konnte. Ein vollständiger Lappenverlust trat nicht auf. Eine Patientin verstarb 2 Wochen postoperativ an den Folgen einer Hirnmetastase. In 2 Fällen war nach Rippen- bzw. Sternumteilresektion eine Netz- bzw. Palacosimplantation notwendig. $\mathrm{Zu}$ einer Instabilität der Thoraxwand kam es in keinem Fall, die Hebedefekte heilten in 6 von 8 Fällen primär ein (1 Nabelnekrose, 1 Sekundärnaht am Unterbauch), in 1 Fall mußten mehrmalige Serompunktionen durchgeführt werden, eine Herniation oder Bauchwandschwäche trat bei keiner Patientin auf. Schlussfolgerung: Die Ergebnisse dieser Untersuchung zeigen, daß mit Hilfe mikrochirurgischer Techniken und in interdisziplinärer Kooperation mit Thoraxchirurgen auch sehr ausgedehnte Thoraxwanddefekte mit kurativem oder palliativem Therapieansatz behandelt werden können. Den großen Vorteil bei der Verwendung von multipedikulären freien DIEP- Lappen sehen wir in der primären Defektdeckung mit Eigenhaut ohne Verwendung von Spalthaut, wie es bei einem Latissimuslappen notwendig wäre, sowie in der geringen Hebedefektmorbidität bei gleichzeitig hoher Sicherheit der Lappendurchblutung. In Palliativsituationen wird durch dieses Verfahren ein erheblicher Gewinn an Lebensqualität durch Verbesserung der pflegerischen Situation erzielt.

\section{P4}

Rekonstruktionsmöglichkeiten nach Resektion ausgedehnter Thoraxwandrezidive beim Mammakarzinom als palliative Indikation

*Daniel Druecke, Stefan Langer, Marcus Lehnhardt, Hans Ulrich Steinau, Heinz Herbert Homann

Universitätsklinik für Plastische Chirurgie und Schwerbrandverletzte Handchirurgiezentrum, Operatives Referenzzentrum für

Gliedmaßentumore, BG-Kliniken "Bergmannsheil»,

Ruhr Universität Bochum, Deutschland

Zielsetzung: Die Häufigkeit für das Auftreten von Thoraxwandrezidiven wird für nodalpositive und nodalnegative Frauen in der Literatur in Abhängigkeit von der Tumorgröße und dem Grading unterschiedlich, wenn überhaupt, angegeben. Insgesamt dürfte das Risiko für ein Lokalrezidiv derzeit aus allen korrekt behandelten Gruppen heraus bei 10-33\% liegen. Liegen große exulzerierte Rezidive vor, kann auch unabhängig von Fernmetastasen eine palliative Indikation zur Resektion bestehen. Materialien und Methoden: Von 1998-2003 erfolgte bei insgesamt 59 Patientinnen mit ausgedehnten Thoraxwandrezidiven in unserer Klinik eine allschichtige 
Thoraxwandteilresektion mit einzeitiger Rekonstruktion. Bei 32 Pat. lag eine palliative Indikation (M1-Status, exulzeriert) vor. Rekonstruktionsverfahren in der palliativen Gruppe: Gestielte Latissimus-dorsi-Insellappenplastiken $(\mathrm{n}=12)$, gestielte Latissimus-dorsi-Schwenklappenplastiken $(\mathrm{n}=$ 8), gestielte Pectoralis-major-Insellappenplastiken $(n=4)$, gestielte TRAMLappenplastiken $(\mathrm{n}=3)$, Zyklopen-Brustbildungen $(\mathrm{n}=2)$, Spalthauttransplantationen $(n=3)$ (keine Thoraxwandresektion). Ergebnisse: Die mittle re stationäre Behandlungsdauer lag bei 19 Tagen. Bei allen allschichtigen Thoraxwandresektionen erfolgte vor der Weichgewebsrekonstruktion die Stabilisierung mittels Prolenenetz. Bei allen Pat. konnte, mit dem jeweils gewählten Verfahren, eine adäquate Behandlungsdauer und zeitgerechte Entlassung mit stabilen Wundverhältnissen erzielt werden. Das mittlere Überleben nach Entlassung betrug 18 Monate. Nennenswerte Komplikationen lagen nur bei 2 Pat. in der Notwendigkeit einer verlängerten Nach beatmungsperiode von jeweils 10 Tagen bei dem Auftreten einer postoperativen Pneumonie bei radiogener Lungenfibrose vor. Zusammenfassung Unter der Beachtung bestimmte Behandlungskriterien ergeben sich insgesamt, auch bei aufwendigen Resektionen und Rekonstruktionen von Thoraxwandrezidiven eines Mammakarzinoms als palliative Indikation, geringe Komplikationsraten. Ziel dieser Behandlungsstrategie ist es, über die Verringerung der Tumorlast und Erzeugung einer stabilen Weichgewebsbedeckung, eine Verbesserung der Lebensqualität zu erzielen. Die Verläufe aus dem vorgestellten Patientengut unserer Klinik stützen diese Auffassung.

\section{P5}

Ästhetische Mammachirurgie bei Patientinnen mit Anisomastie und Poland-Syndrom - Erfahrungen im eigenen Patientengut

*Matthias Föhn, Nestor Torio Padron, Arash Momeni, G. Björn Stark Abteilung Plastische und Handchirurgie Universitätsklinikum Freiburg, Freiburg, Deutschland

Das Poland-Syndrom ist eine rare angeborene Fehlbildungsstörung der oberen Extremität und der Brust. Weltweit tritt es bei zirka 1:30000 Geburten auf. Die Ursache ist noch nicht vollständig geklärt, jedoch ist ein Verschluß der Arteria subclavia am 46. Tag der Schwangerschaft als Grund anzuneh men. Männliche Neugeborene erkranken doppelt so häufig wie weibliche. Ebenso läßt sich eine Dominanz zur rechtsseitigen Ausprägung feststellen. Wir berichten über 20 Patientinnen mit Anisomastie und tubulären Brüs ten (davon 3 mit Polandsyndrom), die durch Implantation von Silikonprothesen und Neuformung der Brustkontur behandelt wurden. Die Prothesen wurden hierbei 17 Mal über einen submammären Zugang, zwe Mal über einen periareolären Zugang und einmal endoskopisch-assistiert über einen axillären Zugang implantiert. In einigen Fällen wurde eine angleichende Straffungsoperation der Gegenseite durchgeführt.

Der intra- und postoperative Verlauf war in allen Fällen komplikationslos Die ästhetischen Resultate können als gut bis sehr gut bezeichnet werden Anhand eines Fragebogens sollen die Patientinnen Auskunft über ihre sub jektive Zufriedenheit hinsichtlich des operativen Ergebnisses geben. Ebenso werden die Patientinnen zu eventuellen Änderungen ihres Selbstwertge fühles und ihrer Sexualität befragt. Aufgrund der noch andauernden Auswertung der Fragebögen lassen sich noch keine abschließenden Aussagen diesbezüglich machen. Diese werden jedoch zum Kongreß vorliegen.

P6

\section{Deckung großer chronischer Strahlenschäden im anterolateralen Thoraxbereich}

\section{${ }^{*}$ Robert Hierner ${ }^{1}$, Dirk van Ramdonck ${ }^{2}$}

${ }^{1}$ Univeristätsklinikum Gasthuisberg

Katholische Universität Leuven, Plastische, Rekonstruktive und

Ästhetische Chirurgie, Leuven, Belien

2Univeristätsklinikum Gasthuisberg

Katholische Universität Leuven, Thoraxchirurgie, Leuven, Belgien

Einleitung: Das Prinzip der multidisziplinären Defektsanierung liegt in einem adäquaten Debridement durch die Kollegen der Thoraxchirurgie mit anschliessender plastisch-chirurgischer Defektdeckung. Material und Methode: 12 Patientinnen mit einem chronischen Strahlenschaden nach Bestrahlung eines Brustcarzinoms wurden operativ behandelt. Das Alter der Patientinnen betrug zwischen 54 und 85 Jahre. Bei allen Patientinnen lag radiologische Veränderungen der Rippen, bzw. des Sternums im Bestrahlungsbereich vor. Bei allen Patientinnen wurden mindestens 3 Rippen entfernt, wobei bei 3 Patientinnen zusätzlich auch eine partielle Sternumresektion durchgeführt werden musste.Die Defektdeckung erfolgte bei 2 Patientinnen mithilfe einer gestielten pectoralis major Muskellappenplastik und in 10 Fällen mit einer gestielten myo-kutanen Latissimus dorsi Lappenplastik. In einer retrospektiven klinischen Studie wurden folgende Untersuchungskriterien evaluiert: Operationsdauer, Dauer der postoperativen Intubation, Auftreten einer postoperativen Pneumonie, Wundverhältnisse und Art und Anzahl von Komplikationen. Ergebnisse: Die Operationsdauer betrug zwischen 3,20 und 6,50 Stunden. Eine Umlagerung der Patietinnen zur Defektdeckung war nicht notwendig. Eine direkt postoperative Extubation erfolgte bei 2 Patienten mit pectoralis major Lappenplastik. Die Dauer der postoperativen Intubationsphase betrug zwischen 3 und 24 Tagen. Bei 4 von 12 Patienten trat postoperativ eine Pneumonie auf, woran 1 Patientin auch verstarb. Stabile Wundverhältnisse ohne Wundheilungsstörungen zeigten sich bei 7 Patientinnen. Bei 4 Patienten traten Wundheilungsstörungen auf, die eine zweite Operation nötig machten. Schlussfolgerungen: Die operative Sanierung von grossen Thoraxwanddefekten nach Bestrahlung betrifft vor allem Patientinnen höheren Lebensalters mit oft deutlich Reduziertem Allgemeinzustand. Bei einem Viertel der Patientinnen liegt zusätzlich ein Spinocelluläres Karzinom im Wundbereich vor.

\section{P7}

\section{Thoraxwandrekonstruktion mit dem kontralateralen} Pectoralislappen - Technik und Indikation

\section{*J. Hoch, T. Elsner, A. Stahlenbrecher}

Klinik für Hand-, Brust- und Plastische Chirurgie, Klinikum Neustadt in Holstein, Neustadt in Holstein, Deutschland

Zielsetzung: Thoraxwanddefekte können auf vielfältige Weise verschlossen werden. Gerade bei älteren und multimorbiden Patienten sollte das Verfahren risikoarm, schnell, komplikationslos und sicher durchführbar sein und dabei keine große Hebemorbidität beinhalten. Diese Forderungen erfüllt die kontralaterale Pectoralislappenplastik, bei der die parasternale Lappenstielung eine buchseitenartige Transposition des Muskels auf die kontralaterale Thoraxwand ermöglicht. Methodik: Anhand des Fallbeispieles einer 73jährigen Patientin werden anatomische Grundlagen des Pectoralislappens, operatives Vorgehen und Langzeitergebnis illustriert. Ergebnis/Zusammenfassung: Wenn auch der Möglichkeit einer sicheren Defektdeckung durch die Größe des M. pectoralis Grenzen gesetzt sind, ergibt sich eine Indikation für diese Lappenplastik bei primär exulcerierten Mammacarcinomen oder deren Lokalrezidiven, Radiodermen, instabilen Narben oder Sternumosteitiden und eignet sich besonders bei Multimorbidität und beiderseitgen Befunden an der Thoraxwand.

\section{P8}

\section{Hautsparende Mastektomie und Sofortrekonstruktion mit} Latissimus dorsi Lappenplastik - Indikationen und Resultate

*Jürgen Hoffmann, Eva Böhm, Bernd Bürkle, Julia Müller-Weihrich, Volker Möbus

Frauenklinik/Senologie, Städtische Kliniken

Frankfurt am Main-Höchst, Frankfurt, Deutschland

Ziel: Untersuchung zur onkologischen Sicherheit und der ästhetischen Resultate der autologen Sofortrekonstruktion mit Latissimus dorsi Lappenplastik nach hautsparender Mastektomie. Patienten und Methoden: Von 2001 bis 2004 (Jan.) führten wir bei 54 Pat. mit primärem MammaNpl. als operative Ther. eine hautsparende Mastektomie mit sofortiger, autologer Rekonstruktion mit Latissimus dorsi Lappenplastik durch. Indikationsspektrum: multizentr., invasives $\mathrm{Npl}(\mathrm{n}=19)$, invasives $\mathrm{Npl}$ mit zentralem Tumorsitz $(n=5)$, invasives Npl. $>3 \mathrm{~cm}(n=9)$, invasives Npl. mit ausgedehnter intraductaler Komponente $(\mathrm{n}=12)$, DCIS $>3 \mathrm{~cm}$ oder multizentrisches DCIS $(n=9)$. Bei 11 Pat. erfolgte der Eingriff nach einer primären Chemotherapie (20\%). Eine simultane kontralaterale Angleichung i.S. einer Mastopexie oder Reduktionsplastik wurde 21 Pat. durchgeführt.Ein positiver axillärer Nodalstatus wurde bei 14 Pat. festgestellt (26\%). Einer postoperativen Radiatio wurden 32 Pat. zugeführt (59\%). 
Ergebnisse: Eine Pat. entwickelte 24 Monate postoperativ ein Lokalrezidiv. Bei zwei Pat. traten Fernmetastasen auf (18 bzw. 32 Monate postoperativ). Komplikationen: Infektion 1,Nachblutung mit operativer Revision 1, Fettgewebsnekrosen 2,Serome der Entnahmestelle traten bei 52\% der Pat. auf. Ästhetische Ergebnisse: Sehr gut 46 (85\%), Gut 6 (11\%) Schlecht bzw. korrekturbedürftig 2 (4\%). Der zeitliche Ablauf einer nachfolgenden adjuvanten Therapie wurde bei keiner Pat. durch den Eingrif verzögert.Zusammenfassung: Die hautsparende Mastektomie mit Sofortrekonstruktion mit Latissimus dorsi Lappenplastik ist nach dem oben genannten Indikationsspektrum eine onkologisch sichere, komplikationsarme Operationsmethode in der primären Therapie des Mammakarzinoms mit sehr guten Ästhetischen Resultaten

\section{P9}

\section{Einsatz der handgeführten,sonographisch gesteuerten Vakuumbiopsie zur Diagnostik und Resektion benigner "exzisionswürdiger» Mammatumoren in der klinischen Routine}

*Jürgen Hoffmann, Julia Müller-Weihrich, Eva Böhm, Bernd Bürkle, Volker Möbus

Frauenklinik/Senologie, Städtische Kliniken Frankfurt am MainHöchst, Frankfurt, Deutschland

Ziel: Bewertung einer minimal-invasiven Methode in der klinischen Praxis zur Diagnostik und Resektion benigner Mammatumoren ( hand held Mammotome) in Lokalanästhesie unter sonographischer Kontrolle. Patienten und Methoden: Seit 3 Jahren setzen wir die sonographisch gesteuerte Vakuumbiopsie in Lokalanästhesie zur Diagnosebestätigung und Resektion benigner,exzisionswürdiger Mammatumoren bis zu einer Größe von $2,5 \mathrm{~cm}$ ein.Bislang wurden bei 245 Pat. 321 benigne imponierende exzisionswürdige Läsionen nach der oben genannten Methode entfernt.Das Vakuumbiopsiesystem erlaubt die Resektion der Läasion im Sinne einer minimal-invasiven Prozedur in Lokalanästhesie und die Diagnosebestätigung über die histopathologische Aufarbeitung des entnom menen Gewebes. Ergebnisse: Die durchschnittliche Dauer des Eingriffs btrug 18 Minuten bei Verwendung der 11G-Nadel und 12 Minuten bei verwendung der 8G-Nadel.Bei 46 Pat. wurde aufgrund vorhandener sonographischer Malignitätskriterien primär eine 14G Core-Biopsie durchgeführt. ERST NACH Malignitätsausschluß kam hier die Vakuumbiopsie zur Resektion zum Einsatz. Eine Komplettrsektion nach sonographischen Kriterien wurde bei 308 Läsionen erreicht (96\%). Diese Ergebnisse wurden über eine obligatorische, sonographische Verlaufskontrolle bestätigt.Bei 3 Patientinnen wurde postoperativ eine Punktion bei Hämatom bildung mit max. $15 \mathrm{ml}$ blutigem Sekret notwendig. Histopathologische Resultate: Fibroadenom 139, Papillom 37, Lipom 6, Adenomyoepitheliom 1, tub. Adenom 1, fibr. MP 28, Fibr.-cyst. MP 48, skler. Adenose 12, narbige Veränderungen 42, nod.Fasziitis 1, Gynäkomastia tubularis 4, DCIS 1 (Core-Papillom), invasiv. Ca 1 (Core DIN 1b). Zusammenfassung: Die Vakuumbiopsie (Hand held Mammotome) unter sonographischer Führung in Lokalanästhesie ist eine sichere, komplikationsarme, minimal-invasive Methode zur Resektion und Diagnosebestätigung benigner, exzisionswürdiger Mammatumoren.

\section{P10}

\section{Das exulzerierte Mammakarzinom - eine chirurgische Herausforderung}

*Walfried Janus, Mohei Qadduri, Helmut Zühlke

Klinik für Allgemein-, Viszeral- und Gefäßchirurgie des

Krankenhauses der Paul-Gerhardt-Stiftung Wittenberg, Wittenberg, Deutschland

Zielsetzung: Das Mammakarzinom ist die häufigste Krebserkrankung der Frau. In Deutschland erkranken etwa 46000 Frauen jährlich an Brustkrebs. Trotz aller Bemühungen der Verbesserung der Früherkennung und Aufklärung in der Bevölkerung kommen immer wieder Patientinnen mit bereits fortgeschrittenen exulzerierten Befunden in die Behandlung. Anhand von Fallbeispielen wird das auf die Situation der Patientinnen abgestimmte Behandlungskonzept dargestellt. Materialien und Methoden: Vom 01.01.1998-31.12.2003 wurden insgesamt 327 Patienten mit einem Mammakarzinom behandelt und operiert. Bei 13 Patienten (3,9\%) lag ein exulzerierter Befund vor. Ergebnis: In das Behandlungskonzept des primär inoperablen Mammakarzinoms gehören die primäre systemische Chemotherapie (PST) und die Führung der Patientin zur Operation. Das operative Spektrum umfasst die Palette der radikalen onkologischen Resektion einschließlich der plastischen Versorgung. Schlussfolgerung: Das exulzerierte Mammakarzinom ist heute eine seltene Entität. Primäre systemische Chemotherapie, Mastektomie mit plastischer Versorgung und Nachsorge sind Eckpfeiler der Behandlung.

P11

\section{Intraoperative Abklatschzytologie zur Sentinel Lymphknoten Diagnostik - ein Routineverfahren?}

${ }^{*}$ Matthias Kalder ${ }^{1}$, Anja Milkereit ${ }^{1}$, Sabine Klemt ${ }^{1}$, Wibke Alt ${ }^{2}$, Roland Moll ${ }^{2}$, Uwe Wagner ${ }^{1}$, Ute-Susann Albert ${ }^{1}$

1Klinik für Gynäkologie, Gynäkologische Endokrinologie und Onkologie der Philipps Universität, Marburg, Deutschland 2Institut für Pathologie der Philipps Universität, Marburg, Deutschland

Zielsetzung: Kann die intraoperative Abklatschzytologie den Schnellschnitt im Rahmen der Sentinel Lymphknoten (SLN) Diagnostik beim Mammakarzinom zuverlässig ersetzen? Materialien und Methoden: Während der Trainingsphase für die Klinische Interdisziplinäre Sentinel Lymphknoten Studie (KISS) beim Mammakarzinom, wurden von 135 SLN bei 66 Patientinnen neben dem klassischen Schnellschnitt zusätzlich zytologische Abklatschpräparate, gefärbt nach Papanicolaou und Diff Quik angefertigt. Vier Untersucher verschiedenen Ausbildungsstandes klassifizierten die Abklatschpräparate in maligne, benigne, zweifelhaft und nicht auswertbar. Alle SLN wurden nach dem Schnellschnitt routinemäßig histologisch und immunhistochemisch aufgearbeitet. Ergebnisse: $89 \%$ (23/26) der histologisch gesicherten SLN Metastasen konnten sowohl im Schnellschnitt als auch vom erfahrenen Untersucher im zytologischen Abklatschpräparat identifiziert werden. In Abhängigkeit von der Erfahrung des Untersuchers variierte die Sensitivität der Abklatschzytologie zwischen 65\% (17/26) und 89\% (23/26), die Spezifität zwischen 81\% (79/97) und 93\% (90/97) und die Gesamtgenauigkeit zwischen 78\% (96/123) und 92\% (113/123). Die Sensitivität des Schnellschnitts lag bei $89 \%(23 / 26)$, Spezifität bei $100 \%$ und die Gesamtgenauigkeit bei $98 \%$ (120/123). Zusammenfassung: Die intraoperative SLN Diagnostik mittels Abklatschzytologie ist eine schnelle und zuverlässige Methode in der Hand des erfahrenen Untersuchers. Aufgrund der höheren Spezifität bleibt die Schnellschnittdiagnostik der Abklatschzytologie in der Routine überlegen. Die Abklatschzytologie stellt aber mit ihrem geringen Material- und Zeitaufwand eine geeignete Alternative in Regionen mit fehlender Schnellschnittlogistik dar.

P12

\section{Ein Algorithmus zur Mammarekonstruktion}

${ }^{*}$ Eva M. Lang ${ }^{1}$, Christoph Andree ${ }^{1}$,Elmar Stickeler ${ }^{2}$, Jörg Borges ${ }^{1}$, G. Björn Stark ${ }^{1}$

${ }^{1}$ Abteilung f. Plastische- und Handchirurgie,

Albert-Ludwigs-Univeristät, Freiburg, Deutschland

${ }^{2}$ Department Universitätsfrauenklinik, Albert-Ludwigs-Universität, Freiburg, Deutschland

In der Therapie des Mammakarzinoms spielt die Entscheidung über die geeignete Art und den Zeitpunkt der Mammarekonstruktion eine entscheidende Rolle. Anhand eines Logarhythmus sollen in Abhängigkeit von der Ausgangssituation die Entscheidungskriterien dargestellt werden, die sich im Rahmen des zertifizierten Brustzentrums bewährt haben.

Nach einer Ablatio mammae ist die Art der Rekonstruktion von verschiedenen Parametern abhängig, wie Tumorstadium, Art des Tumors, Wunsch der Patientin.

$\mathrm{Zu}$ entscheiden ist zwischen

1. einer Rekonstruktion mit alloplastischem Material oder mit Eigengewebe

2. Soll der Brustwiederaufbau primär, d.h. in einer Operation mit der Ablatio erfolgen, oder sekundär zu einem späteren Zeitpunkt?

3. Welche Rolle spielt der Wunsch der Patientin? 
Ad 1.: Rekonstruktion mit Eigengewebe: prinzipiell bei allen möglich. Rekonstruktion mit alloplastischem Material: mit oder ohne vorhergehende Expanderimplantation: bei allen möglich außer: bei Z. n. Radiatio oder geplanter Radiatio

Ad 2.: Primärer oder sekundärer Aufbau: angestrebt wird die primäre Rekonstruktion, außer: unsichere R0 Resektion oder inflammatorisches Karzinom.

Ad 3.: Der Wunsch der Patientin entscheidet unter den angegebenen Bedingungen über die Art des Materials.

Auch ein fortgeschrittenes Tumorstadium und eine gegebenenfalls begrenzte Lebenserwartung stellen keine Kontraindikation für eine Mam marekonstruktion dar, wenn dies die Patientin wünscht und ihr Allgemeinzustand es zuläßt.

Dieser standardisierte Algorhythmus soll einerseits die Therapieentscheidung und die individuelle Beratung der Patientinnen erleichtern und andererseits eine Hilfe in der interdisziplinären Kommunikation darstellen.

\section{P13}

Der freie Siea-Diep-Kombinationslappen zur Deckung grosser Gewebe-oder Volumendefekte

*Martina Mayr, Charlotte Holm-Jakobsen, Eugen Höfter, Milomir Ninkovic

Abteilung für Plastische-, Rekonstruktive und Handchirurgie,

Zentrum für Schwerbrandverletzte, Städtisches Krankenhaus

München Bogenhausen, München, Deutschland

Einleitung: Seit Mitte der 90er Jahre gilt der am Perforatorgefäß der A. epigastrica inferior gestielte DIEP-Lappen in der Brustrekonstruktion nach Mammakarzinom als wertvollste Alternative zum musculocutanen TRAMLappen. Seine Vorteile liegen in einer geringeren Spendermorbidität, sowie kürzeren Erholungszeiten für den Patienten. Leider erwies sich die Durch blutung und somit die Überlebensrate im contralateralen äußeren Antei des Lappens (Zone IV) als unsicher, so dass dieser Teil in der Regel nich zur Rekonstruktion verwendet wird. Diese Tatsache limitiert den Einsatz des DIEP-Lappens zur Wiederherstellung großflächig ausgedehnter Defek te, oder einer voluminöseren Brust, wenn dies von der Patientin gewünscht wird. Ziel: Ziel war es zu überprüfen, ob bei Verwendung des DIEP-Lappens durch den Erhalt der contralateralen A. epigastrica superficialis (SIEA) die Durchblutung der Zone IV gesichert werden kann, um somit einen noch großflächiger verwendbaren Gewebeersatz zu erhalten. Material und Methode: 4 Patientinnen mit ausgedehnten Gewebe- oder Volumendefekten werden vorgestellt. Zur Defektdeckung oder Volumenrekonstruktion wurde die Kombination eines mikrochirurgisch frei am Perforatorgefäß der A. epigastrica inferior transferierten DIEP-Lappens mit Erhalt und parallelem Anschluß der contralateralen A. epigastrica superficialis (SIEA) verwendet. Ergebnisse: Bei allen Patienten konnte das gesamte vom Bauch gewonnene Gewebe zur Defektdeckung und Rekonstruktion verwende werden. Eine Zone IV, wie beim DIEP-Lappen bekannt, musste nicht verworfen werden. Mit Hilfe der dynamischen Laser-Fluoreszenz-Videoangiographie konnte die Durchblutung des Lappens in Zone IV dargestellt werden. Schlussfolgerung: In erfahrenen Händen bietet der freie mikrochirurgische Transfer eines SIEA-DIEP-Kombinationslappens dort eine wertvolle Alternative, wo der gesamte Unterbauchlappen (inklusive Zone IV) zur Defektdeckung oder Volumenrekonstruktion erforderlich ist.

\section{P14}

\section{Signifikante Verminderung postoperativer Schmerzen und axillärer Lymphserome durch die ADAM Technik der axillären Dissektion im Rahmen brusterhaltender Operationen ohne separaten Zugang}

*Stefan Paepke, U. Schwarz-Boeger, A. Humbert, M. Kiechle, V. R. Jacobs

Frauenklinik des Klinikums rechts der Isar, Technische Universität, München, Deutschland

Einleitung: Der Trend zur Minimalisierung operativer Eingriffe setzt sich in der Mammachirurgie durch. Die ADAM-Technik (Axillary Dissection with Access Minimized) verzichtet unter Zuhilfenahme endoskopischer Instrumente oder Leuchtspiegel gänzlich auf den separaten Axillazugang bei brusterhaltenden Operationen (Segment- und Quadrantenresektion, tumoradaptierte Reduktionsplastik, Hemimastektomie) unabhängig von der Tumorlokalisation. Material und Methoden: Nach initialer Machbarkeitsstudie $(n=23)$ wurden in einer parallelen Beoabachtungsstudie 47 nach ADAM operierte Patientinnen 49 mit konventioneller axillärer Dissektion (KAD) gegenübergestellt. Auswertung: Onkologische Sicherheit (Anzahl entfernter Lymphknoten, Erfolg der SLNB) - Postoperative Morbidität (Seromhäufigkeit, Schmerzen, Armmobilität) -·Kosmetisches Resultat. Ergebnisse: Die Operationsdauer für die ADAM-Technik (Schnitt-Naht-Zeit einschliesslich Schnellschnittbestimmung der Schnittränder und Sentinel-LN) betrug 104,11 min (54,16-154,05), für die KAD 116,36 (69,05-163,67). Hinsichtlich Tumorgrösse, SLNB, entfernter und positiver Lymphknoten, prä-postoperativer Hb-Differenz und Hämatomausprägung waren keine Unterschiede zwischen beiden Operationstechniken. Die Anzahl der postoperativen Lymphserome ist nach der ADAMTechnik signifikant erniedrigt ( $\mathrm{p}=0,001$ Mann-Whitney-Test); ebenso die postoperative Schmerzsymptomatik (keine 28,2 vs. $18,0 \%$; wenig 47,8 vs. $64,0 \%$; starke Schmerzen 2,17 vs. 2,46\%). Schlussfolgerungen: Die onkologische Sicherheit ist gewährleistet, die Kombination des Eingriffs mit der SLNB problemlos möglich. Die Operationstechnik ist bei nodal negativen Patientinnen und auch ausgedehntem Lymphknotenbefall sicher durchführbar. Die Armbeweglichkeit ist uneingeschränkt, die voll-physiotherapeutische Beübung beginnt am 1. postoperativen Tag. Die Armmobilität ist durch den Wegfall der äusseren Narbe signifikant verbessert, die Entwicklung von Lymphseromen durch den Erhalt des subcutanen Lymphabflusses klinisch signifikant reduziert. Das kosmetische Ergebnis ist absolut vergleichbar, eine Schnittverlängerung zur Gewährleistung eines sichereren Zugangs zur Axilla war nicht notwendig.

\section{P15}

\section{Sonografische Identifikation und Feinnadelmarkierung des Sentinel Lymphknoten beim T1 und kleinen T2 (<3 cm) Mammakarzinom im Vergleich zur Patentblau- und Tc99- Markierung bei 40 Patientinnen}

*Birgit Plattner, Markus Niemeyer, Volker R. Jacobs, Marion Kiechle Frauenklinik, Technische Universität München, Deutschland

Zielsetzung: Die Sentinellymphonodektomie beim Mammakarzinom stellt ein minimal-invasives Operationsverfahren mit geringer Morbidität dar. Die etablierten Detektionsverfahren des Sentinellymphknotens (SLN) sind die Patentblaumarkierung sowie die radioaktive Markierung mit Tc99. Die Sensitivität beider Methoden liegt bei 82-100\%. Ziel dieser prospektiven Studie ist die Evaluation der sonografischen Darstellung des SLN, des zeitlichen Aufwands, der Einfluß des Körpergewichtes (BMI) für das Auffinden des SLN sowie die Übereinstimmung mit den üblichen Markierungsverfahren. Methode: Bei 40 Patientinnen $(n=40)$ erfolgte im Zeitraum zwischen August 2003 und Mai 2004 nach stanzbioptischer Sicherung des cT1-cT2 $(<3 \mathrm{~cm}) \mathrm{cN} 0$ Mammakarzinoms die Sentineldarstellung mittels sonografischer Nadelmarkierung sowie Patentblau oder Tc99. Die sonografische Markierung und das intraoperative Aufsuchen des SLN erfolgte durch einen einzigen erfahrenen Ultraschaller bzw. Operateur. Ergebnisse: Bei 34 von 40 Patientinnen konnte der SLN sonografisch dargestellt und markiert werden und zeigte eine Übereinstimmung mit der Blau- oder Tc99-Markierung. Die Sensitivität der Methode liegt bei 85\%. Die falsch negative Rate entspricht der Literatur mit 8\%. Die Zeit bis zum Auffinden des SLN sowie dessen Markierung betrug im Durchschnitt 9 Minuten. Die Ergebnisse waren unabhängig vom BMI der Patientinnen. Zusammenfassung: Mit der ultraschallgesteuerten Identifikation und Markierung des SLN steht ein kostengünstiges Verfahren mit geringer Morbidität für die Patientinnen zur Verfügung. In der Hand des Geübten stellt dieses Verfahren eine zusätzliche Möglichkeit der Sentineldarstellung neben Patentblau und Tc99 dar, vor allem an Institutionen mit geringeren Ressourcen. Weitere Studien mit größeren Patientenzahlen sind nötig, um die hier vorgestellten Daten zu bestätigen. 
P16

Können integrierte Versorgungskonzepte zu einer messbaren Verbesserung der regionalen Versorgungsqualität beim Mammakarzinom führen?

*Katy Roterberg 1, 2, Marcus Blumenröther ${ }^{1,3}$, Thomas Darnedde ${ }^{1,4}$, Thorsten Kühn ${ }^{1,2}$

${ }^{1}$ Brustzentrum Gifhorn, Gifhorn, Deutschland

${ }^{2}$ Frauenklinik Kreiskrankenhaus, Gifhorn, Deutschland

${ }^{3}$ Radiologische Gemeinschaftspraxis, Gifhorn, Deutschland

${ }^{4}$ Pathologisches Institut, Gifhorn, Deutschland

Zielsetzung: Die Zielsetzung von Brustzentren und integrierten Versorgungsmodellen liegt in der verbesserten Kooperation verschiedener Fachdisziplinen sowie der Vernetzung von ambulanten und stationären Leistungsträgern, Interessenvertretern und Selbsthilfegruppen. Inwieweit dieses Konzept die regionale Versorgungsqualität für das Mammakarzinom beeinflussen kann, ist bisher wenig untersucht. Material und Methode: Seit 2001 sind am interdisziplinären Brustzentrum Gifhorn die Anforderungen an eine spezialisierte Brustklinik in der Routine etabliert. Die Qualitätsindikatoren der EUSOMA werden seit 2002 systematisch erfasst und ausgewertet. Um die Qualität der regionalen Versorgungsstruktur zu erfassen, haben wir die Tumorstadien sowie die Raten brusterhaltender Verfahren aus den Jahren 2002 und 2003 ausgewertet und miteinander verglichen. Ergebnisse: Die Rate prämaligner Läsionen betrug im Jah 2002 4,8\% und im Jahr 2003 12,6\%, die Rate an pT1a-Tumoren 0,8\% bzw. 7,2\% und der Anteil nonpalpabler Karzinome 17,0\% bzw. 27,5\%. Die Rate der pT1-Tumoren veränderte sich von 58,5\% auf 63,8\% und der Anteil nodalnegativer invasiver Karzinome von 63,2\% auf 64,1\%. Dagegen fiel die Rate fortgeschrittener Tumoren (T2) von 30,0\% auf 17,4\%. Die Rate brusterhaltender Operationen (pT1 und pT2) konnte von 61,4\% auf $74,0 \%$ gesteigert werden. Der Anteil an Sentinel-Node-Biopsien stieg von 19,5\% auf 39,1\%. Zusammenfassung: Die Implementierung von Brustzentren sowie die systematische Erfassung von Qualitätsindikatoren kann dazu beitragen, die regionale Versorgungsstruktur für das Mammakarzinom darzustellen und erkennbar zu verbessern.

\section{P17}

\section{Die interdisziplinäre Rekonstruktion der Brust durch mikrochirurgische TRAM Plastik}

*Peter Scheler ${ }^{1}$, Franz-Peter Uckmann ${ }^{2}$, Alexander Dengler ${ }^{1}$, Gerald Hoffmann ${ }^{1}$

${ }^{1}$ Frauenklinik St. Josefs-Hospital, Wiesbaden, Deutschland ${ }^{2}$ Klinik für Thorax und Gefässchirurgie, St. Josefs-Hospital Wiesbaden, Deutschland

Zielsetzung: Die primäre oder sekundäre Rekonstruktion der Brust kann durch den Einsatz alloplastischer Materialien, durch autologe Verfahren, oder durch Kombination beider Techniken durchgeführt werden. Die autologe Rekonstruktion der Brust unter Einsatz mikrochirurgischer Techniken stellt ausserhalb plastisch-chirurgischer Fachabteilungen eine besondere Herausforderung dar. Untersucht werden sollte daher inwieweit innerhalb einer primär gynäkologisch geprägten senologischen Fachabteilung durch die interdisziplinäre Zusammenarbeit mit der Abteilung für Thorax und Gefässchirurgie ein routinemässiger Einsatz mikrochirgischer Techniken möglich ist. Materialien und Methoden: Im Zeitraum 8/02 bis 5/04 wurde bei insgesamt 21 Patientinnen eine Rekonstruktion der Brust durch mikrochirurgischen TRAM Lappen durchgeführt (unilateral $n=20$, bilateral $n=1$ ). Alle Eingriffe wurden von demselben Team, bestehend aus 2 senologisch ausgerichteten Gynäkologen sowie einem Herz-Thorax und Gefässchirurgen durchgeführt. Es wurden jeweils die A./V. epigastrica inferior (in einem Fall auch additiv die V. epigastrica inferior superficialis) mit entweder der A./V. thoracodorsalis oder aber der A./V. mammaria interna anastomosiert. Ergebnisse: Bis auf einen Fall (partielle infektbedingte Hautnekrose)wurden die postoperativen kosmetischen Ergebnisse als gut bis sehr gut eingestuft. Bei 22 Anastomosen mussten 3 Patientinnen revidiert werden (2 Hämatomausräumungen, eine venöse Congestion). Das Alter der jüngsten Patientin betrug 16 Jahre, der ältesten Patientin 68 Jahre. Die stationäre Liegedauer lag zwischen 8 und 14 Tagen, die Operationszeiten variierten zwischen 6 und 9 Stunden $\mathbf{Z u}$ sammenfassung: Bei adäquater Patientinnenselektion stellen mikrochir- urgische Verfahren eine wertvolle Behandlungsoption bei jedoch erheblichem Ressourceneinsatz dar. Voraussetzung für ihren Einsatz sind jedoch umfangreiche mikrochirurgische Kenntnisse. Die enge perioperative interdisziplinäre Kooperation erweitert das Spektrum verfügbarer Behandlungsoptionen.

\section{P18}

\section{Die Adam - Operationstechnik (Axillary Dissection With Access Minimized) reduziert signifikant postoperative Lymphserome in der brusterhaltenden Therapie}

*Ulrike Schwarz-Boeger, Stefan Paepke, Melanie Zacherl, Alexandra Humbert, Marion Kiechle, Volker R. Jacobs Frauenklinik, Technische Universität München, München, Deutschland

Zielsetzung: In der Brustchirurgie gibt es einen Trend zu weniger Invasivität. Eine neue Operationstechnik (ADAM) bei BET macht die Sekundärinzision in der Axilla überflüssig. Die Axilladissektion erfolgt nach Entfernung des Brusttumors von der Primärinzision aus. Zur Unterstützung werden endoskopische Instrumente bzw. Leuchtspiegel eingesetzt. Wir verglichen zwei verschiedene OP-Verfahren, ADAM vs. konventionelle Axilladissektion, auf postoperative Morbidität, Schmerzen, onkologische Sicherheit und kosmetisches Resultat. Materialien und Methoden: Von 06/03-03/04 führten wir eine prospektive, vergleichende Studie durch. Beide Techniken wurden gegebenenfalls mit Sentinel Lymph Node Biopsy (SLN) kombiniert. Die ADAM-Technik wurde aus allen vier Brustquadranten durchgeführt unabhängig vom LK-Status. Insgesamt wurde bei 47 Patienten eine OP nach dem ADAM-Konzept und bei 50 Frauen eine konventionelle Axilladissektion durchgeführt und die postoperativen Ergebnisse miteinander verglichen. Statistische Signifikanz wurde mit dem Fisher- und Mann-Whitney-U-Test überprüft. Ergebnisse: Die demographischen Daten beider Patientengruppen waren vergleichbar. Das Durchschnittsalter war 61,6 Jahre (48-74). Die Operationsdauer für ADAM (Schnitt-Naht-Zeit einschließlich Schnellschnitt und SLN) war 104,1 min (54,2-154,1) und für konventionelle Axilladissektion 116,4 min $(69,1-163,7)$. Entfernte bzw. positive LKs, Tumorgrösse und prä/postoperativer Hb-Abfall und axilläre Hämatome waren in beiden Gruppen gleich; axilläre Lymphserome waren dagegen hochsignifikant weniger bei ADAM $(\mathrm{p}<0,01)$. Das kosmetische Ergebnis war in beiden Gruppen gleich gut. Klinisch zeigten die ADAM-Patienten bereits direkt postoperativ eine bessere, oft uneingeschränkte Armbeweglichkeit. Zusammenfassung: Die ADAM-Technik ist sicher und komplikationslos durchführbar auch nach onkologischen Kriterien. Aufgrund geringerer Invasivität bleiben Lymphgefässe unterhalb der Haut in der Axilla intakt. Hieraus resultiert statistisch signifikant eine reduzierte axilläre Serombildung. Klinisch signifikant ist die erheblich reduzierte Armmorbidität für ADAMPatienten, die sofort nach der Operation erkennbar ist. Das ADAMKonzept ist ein deutlicher Vorteil für unsere Patienten.

\section{P19} "Dual-Plane» Implantat-Position zur Behandlung der
schweren Kapselfibrose mit Adaptation des Hautmantels
durch Reduktionsmastopexie

*Wulf Siggelkow ${ }^{1}$, Heinz Kölbl ${ }^{1}$, Andre Faridi ${ }^{2}$

${ }^{1}$ Universitäts-Frauenklinik Mainz, Mainz, Deutschland

${ }^{2}$ Bereich Senologie/Brustchirurgie der RWTH Aachen, Aachen,

Deutschland

Zielsetzung: Eine Kapselfibrose des Implantatlagers nach Brustrekonstruktionen oder Augmentationen ist nach den bestehenden Therapiemöglichkeiten mit einer hohen Rezidivrate behaftet. Die vorliegende Studie beschreibt eine operative Therapiemöglichkeit der symptomatischen Kapselfibrose nach subglandulärer Implantataugmentation und gleichzeitig bestehender Ptose der Brust. Material/Methoden: Gegenstand der Studie sind 14 Patientinnen mit symptomatischer Kapselfibrose. In $11 \mathrm{~Pa}-$ tientinnen erfolgte der erste Implantatwechsel nach einer durchschnittlichen Liegezeit von 96 Monaten und in dreien der zweite. Das neue Implantat wurde in einer «Dual-plane" Position implantiert, d.h. zum Teil retropektoral und zum Teil subglandular. Die Operation wurde mit einer 
Korrektur der glandulären Ptose kombiniert. Jede dieser Operationen wurde einzeitig durchgeführt. Folgende Schritte gehörten zu jeder Operation: Entfernung des alten Implantates und der Implantatkaspel, Präparation einer kaudalen Hautbrücke durch Deepithelialisierung oberhalb der Submammarfalte, Ablösen des kaudalen Ansatzes des M. pectoralis major und Formung einer neuen Implantatloge durch Verbindung des kaudalen Pektoralisrandes mit dem kranialen Abschnitt der Hautbrücke über dem neuen Implantat («Dual-Plane») und Adaptation des Weichteil/ Hautmantels über dem Pektoralis im Sinne der Reduktionsmastopexie. Der Erhalt der Mamille erfolgte durch kraniale/kraniomediale Stielung. Ergebnisse: Alle Patientinnen wurden bis zu 36 Monate unter Erfassung aller operationsbedingten Komplikationen nachbeobachtet. Kein Implantat musste revidiert werden, es traten lediglich geringe Komplikationen auf. In diesem Zeitraum wurde keine erneute Kaspelfibrose beobachtet. Zusammenfassung: Die vorgestellte Operation bietet eine einzeitige Therapieoption in Fällen schwerer Kapselfibrose und glandulär ptoti scher Brust durch Optimierung des Weichteilmantels über dem Implantat und Adaptation des Hautmantels.

\section{P20}

In vitro Analyse modifizierter Silikonoberflächen von Silikon-

\section{Brustimplantaten}

*Wulf Siggelkow ${ }^{1}$, Heinz Kölbl ${ }^{1}$, Andre Faridi ${ }^{2}$

${ }^{1}$ Universitäts-Frauenklinik Mainz, Mainz, Deutschland

${ }^{2}$ Bereich Senologie/Brustchirurgie RWTH Aachen, Aachen,

Deutschland

Zielsetzung: In der vorliegenden Untersuchung wurden die Eigenschaften hydrophilisierter Silikonoberflächen handelsüblicher Brustimplantate nach kovalenter und adsorptiver Bindung von ExtrazellularmatrixBestandteilen wie Fibronektin und GRGDS auf der Implantatoberfläche in einem Zellkulturmodell analysiert. Die zelluläre Reaktion auf modifizierte Oberflächen wurde mit denen unbehandelten Silikons verglichen. Material/Methoden: Unterschiedlich modifizierte Oberflächenfolien von Silikonbrustimplantaten wurde nach 24 Stunden, 5 Tagen und 7 Tagen im Zellkulturversuch analysiert. Für die Versuche wurden Mausfibroblasten (L 929) verwendet. Der Ausschluss eines zytotoxischen Einflusses des Materials auf die Zellkulturen wurde sowohl im direkten als auch im indirekten Kontakt durchgeführt. Vitalitätsanalysen sowie der qualitative Nachweis der Zellproliferation auf unterschiedlichen Silikonoberflächen wurden mit Fluoreszindiazetat und Ethidiumbromid geführt. Darüber hinaus wurden morphologische Eigenschaften der Zellen an der HE-Färbung beschrieben. Die quantitative Zellanalyse erfolgte nach Resuspension mit dem XTT-Test. Ergebnisse: Toxische Einflüsse der Materialien auf die Zellkulturen konnten sowohl für modifizierte Oberflächen als auch für unbehandeltes Silikon ausgeschlossen werden. Wir fanden eine abgestufte Verbesserung der Zellmorphologie, definiert durch die zellulären Ausbreitungseigenschaften auf dem Material und die Morphologie in Abhängigkeit von der Oberflächenmodifikation. Die besten Ergebnisse fanden wir für kovalent gebundenes Fibronectin und GRGDS auf der Silikonoberfläche verglichen mit Polyacrylsäure oder unbehandeltem Silikon. Schlussfolgerungen: Im Zellkulturversuch scheint die kovalente Immobilisation von hydrophobem Silikon geeignet, die initialen Interaktionen zwischen dem Biomaterial und den umgebenden Zellen der Extrazellularmatrix im Sinne einer aktiven Interaktion zu verbessern. Somit wäre in vivo zu prüfen, wieweit die Kopplung von Bestandteilen der Extrazellularmatrix auf Implantatoberflächen die Kompatibilität von Siliko-Brustimplantaten verbessern kann.
P21

Die Anzahl der entfernten axillären Lymphknoten beeinflusst das Risiko für axilläre Rezidive bei nodalpositiven, aber nicht bei nodalnegativen Brustkrebspatientinnen

*Harald Sommer, Wolfgang Janni, Bernd Gerber, Brigitte Rack, Barbara Strobl, Maja Heinrigs, Dorle Rjosk, Christian Schindlbeck Klaus Friese

I. Frauenklinik, Klinikum der LMU, München, Deutschland

Zielsetzung: Die vorliegende Arbeit untersuchte, ob der Verzicht auf eine systematische Axilladissektion das Risiko für das Auftreten von axillären Rezidiven erhöht. Material und Methoden: Bei 3,800 Patientinnen mit einem Mammakarzinom im UICC Stadium I-III wurden in 188 Fällen 1-3 Lymphknoten (LK), in 1184 Fällen 4-9 LK und in 2428 Fällen mindestens 10 LK entfernt. Die prognostische Relevanz der Anzahl entfernter LK wurde durch multivariate Cox Regressionsanalyse untersucht Mediane Nachbeobachtungszeit: 72 Monate. Ergebnisse: Bei 67 Patientinnen $(1,7 \%)$ trat in der Nachbeobachtungszeit ein axilläres Rezidiv auf. Unter den nodalnegativen Patientinnen $(\mathrm{n}=2,667)$ bestätigten sich in der multivariaten Analyse nur die Kovariaten histopathologisches Grading $(\mathrm{P}=0,04, \mathrm{RR} 2,7,95 \% \mathrm{CI})$ und Tumorgröße $(\mathrm{P}=0,03, \mathrm{RR} 2,8$, 95\% CI 1,1-7,1), nicht jedoch Hormonrezeptorstatus und die Anzahl entfernter axillärer $\operatorname{LK}(\mathrm{P}=0,42)$ als unabhängige Prädiktoren für das Auftreten eines axillären Rezidives. Bei nodalpositiven Patientinnen $(\mathrm{n}=1,133)$ fand sich hingegen neben der tumorösen Fixierung der axillären LK ( $\mathrm{P}=0,005$, RR 3,6, 95\% CI 1,5-8,3) eine unabhängige prognostische Relevanz der Anzahl entfernter LK für das Auftreten axillärer Rezidive ( $\mathrm{P}=0,002$, RR 9,9, 95\% CI 2,7-35,3). Zusammenfassung: Während es bei nodalnegativen Patientinnen keine Hinweise für ein erhöhtes regionäres Rezidivrisiko durch den Verzicht auf eine konventionelle axilläre Dissektion gibt, sollte diese bei nodalpositiven Patientinnen zur Vermeidung seltener Rezidive durchgeführt werden.

\section{P22}

Metastase eines Nierenzellcarcinoms als Mammatumor Fallbericht-

*Birgit Stolze, Bettina Dörken, Petra Stüsser, Norbert Golz Frauenklinik, Marien-Hospital, Euskirchen, Deutschland

Patientin: 51-jährige Patientin mit Z.n. Nephrektomie links vor 3 Jahren bei Nierenzellcarcinom.

Z.n. Harnleitermetastasenresektion rechts vor 3 Monaten. Jetzt Mammatumor links bei 6 Uhr von 2x1,5 cm, kein Plateau, keine Peau d'orange.

Therapie: Jetbiopsie, Tumorexcision

Histologie: Lipidreiche, hellzellige Neubildung.

Metastase des klarzelligen Nierenzellcarcinoms.

Therapie (IOK): Nachresektion bei Randständigkeit. Sonst keine weitere Therapie

\section{P23}

Der basal gestielte Drüsenkörperlappen als vielfältig einsetzbare Technik in der Brustchirurgie

Matthias Voigt

Praxis für Plastische Chirurgie Freiburg

Ziel: Häufig kommt es in der ästhetischen und rekonstruktiven Brustchirurgie zu Situationen, in denen Brustvolumen an einer bestimmten Stelle gebraucht wird. Z.B. bei Straffungen und Reduktionsplastiken im Bereich der oberen Quadranten (upper filling), bei der Rekonstruktion der Brust nach brusterhaltender Therapie (BET) und bei der Rekonstruktion von tubulären Brüsten der Typen I und II. Methodik: An perforierenden Gefäßen durch die Pektoralis Muskulatur lässt sich ein Drüsenkörperareal von bis zu $7 \times 7 \times 13 \mathrm{~cm}$ isolieren, welches frei beweglich in den entsprechenden Defekt eingesetzt werden kann. In der Zeit von März 2002 bis Mai 2004 wurde bei 32 Patientinnen der caudal gestielte Drüsenkörperlappen als Technik während einer Brustoperation eingesetzt. Die Operationen im Einzelnen waren vertikale Brustreduktionen $(\mathrm{n}=15)$, vertikale Bruststraffungen $(\mathrm{n}=10)$, Rekonstruktionen nach BET $(\mathrm{n}=3)$ und Re- 
konstruktionen bei tubulären Brüsten Typ I und II $(\mathrm{n}=4)$. Das Volumen des Drüsenkörperlappens wurde in den vorhandenen Defekt eingesetzt. Die Durchblutung des Lappens war immer gewährleistet. Ergebnisse: Bei den Reduktionsplastiken konnte eine gute Projektion in den oberen Quadranten erzielt werden, innerhalb des Nachbeobachtungszeitraums von 1 - 26 (Median 18) Monaten war keine Aussackung (Drop out festzustellen). Die Rekonstruktion der unteren Quadranten bei tubulären Brüsten konnte erreicht werden. Es kam zu einer verzögerten Wundheilung der vertikalen Wunde. Es kam zu keiner Durchblutungsproblemen durch die Lappenplastik. Zusammenfassung: Der basal gestielte Drüsenkörperlappen ist bei ästhetischen und rekonstruktiven Brusteingriffen eine wertvolle zusätzliche Technik, mit der Nachteile der bisherigen Operationsverfahren teilweise beseitigt werden können.

\section{Poster: Langzeitbeobachtung/Lebensqualität}

P24

\section{Subjektiver psychoonkologischer Betreuungsbedarf bei Patientinnen mit Mammakarzinom}

Kai Beckmann ${ }^{1}$, Faouz Allali ${ }^{1}$, *Peter A. Fasching ${ }^{1}$, Michael P. Lux ${ }^{1}$, Ute Kind-Winkler ${ }^{1,2}$, Martina de Zwaan ${ }^{2}$, Matthias W. Beckmann ${ }^{1}$ ${ }^{1}$ Frauenklinik der Universität Erlangen-Nürnberg, Erlangen, Deutschland; ${ }^{2}$ Abteilung für Psychosomatik und Psychotherapie des Klinikums der Universität Erlangen-Nürnberg, Erlangen, Deutschland

Psychoonkologische Begleitung ist eine Standardoption in der Behandlung von Patientinnen mit Mammakarzinom. Zur Qualitätssicherung in der Versorgung der Betroffenen ist vor dem Hintergrund von Zentralisierung der Behandlung des Mammakarzinoms und damit steigender Fallzahl pro Zentrum die optimale Identifikation von Patientinnen mit erhöhtem Bedarf an psychologischer Begleitung notwendig. Verlässliche Indikatoren hierfür könnten eine ökonomische Durchführung psychoonkologischer Versorgung auf hohem Niveau begünstigen.

Um Anhaltspunkte für derlei Indikatoren zu erhalten, wurden an $670 \mathrm{~Pa}$ tientinnen Fragebögen zum subjektiven psychoonkologischen Betreuungsbedarf (SPB) ausgegeben, die zusätzlich Tools zur Messung von Angst, Depression und Lebensqualität (QoL) enthielten. Zur Erfassung des SPB wurde ein Summenscore aus den Antworten auf zentrale Fragen entwickelt.

Der Rücklauf betrug 90,7\%. Die Stichprobe erwies sich als repräsentativ Es zeigte sich, dass Patientinnen jüngeren Alters, mit höherem Bildungsabschluss, erhöhtem Angstniveau oder niedrigerer QoL einen erhöhten SPB haben. Die genannten Parameter erwiesen sich in der Untersuchung als unabhängige Einflussgrößen. Soziale Faktoren (Familienstand, Kinder) sowie Metastastierungsstatus oder Zeitdauer seit der Erstdiagnose hatten innerhalb der Stichprobe keinen signifikanten Einfluss auf den SPB. Unter den Formen psychoonkologischer Betreuung wurden individuelle Einzelsitzungen von den Patientinnen favorisiert.

Die Verlässlichkeit des SPB in der Abschätzung des tatsächlichen psychoonkologischen Betreuungsbedarfs ist umstritten. Der SPB bietet jedoch einen ersten Ansatzpunkt für die Suche nach geeigneten Indikatoren der Notwendigkeit psychoonkologischer Intervention. Eine prospektive Evaluation der in dieser Untersuchung identifizierten Indikatoren wird derzeit in der Betreuung onkologischer Patientinnen durchgeführt.

\section{P25}

\section{Kommunikation innerhalb der Familie nach Mitteilung des BRCA1/2-Mutationsstatus}

Dorothea Gadzicki , Luzie Ursula Wingen ${ }^{1}$ Irmgard Nippert², * Brigitte Schlegelberger ${ }^{1}$ und Mitglieder der Arbeitsgruppe "Humangenetik» des Konsortiums "Familiärer Brust- und Eierstockkrebs" der Deutschen Krebshilfe: C. R. Bartram, D. Beger, K. Bosse, I. Debatin, B. Fischer, U. Froster, D. Gadzicki, T. Goecke, T. Grimm, W. Grote, L. Harder, D. Horn, J. Horst, F. Kreuz, U. Langenbeck, T. Neumann, I. Nippert, P. Propping, D. Schäfer, B. Schlegelberger, D. Schmidt, J. Schröder, R. Siebert, K: Sperling, S. Strenge, W. Vogel, T. Voigtländer, B. Welling ${ }^{1}$ Institut für Zell- und Molekularpathologie, Medizinische Hochschule Hannover, Hannover, Deutschland

${ }^{2}$ Institut für Humangenetik, Arbeitsgruppe Frauengesundheitsforschung, Universitätsklinikum Münster, Münster, Deutschland

Mit dem Ziel, das vom Konsortium «Familiärer Brust- und Eierstockkrebs» der Deutschen Krebshilfe entwickelte Beratungskonzept zu verbessern, wurden 332 Frauen (74\% des Gesamtkollektivs), die das Ergebnis der molekulargenetischen Untersuchung der Brustkrebsgene BRCA1 und BRCA2 vor mehr als sechs Monaten erfahren hatten, in einem strukturierten Telefoninterview befragt. Nahezu alle Frauen sprachen vor (91\%) und nach (98\%) Durchführung des Testes mit ihrem Ehemann oder anderen Familienangehörigen. Dabei wurden weibliche Familienangehörige signifikant häufiger informiert als männliche (Töchter 93\% gegenüber Söhnen $78 \%, \mathrm{p}<0,05 \mathrm{Chi}^{2}$; Schwestern $82 \%$ gegenüber Brüdern $57 \% ; \mathrm{p}<0,05 \mathrm{Chi}^{2}$ ). $10 \%$ aller Frauen wurden von Familienangehörigen gebeten, das Testergebnis nicht mitzuteilen. Trägerinnen einer krankheitsassoziierten Mutation erlebten häufiger Konflikte (22\% gegenüber 3\%; p $<0,05 \mathrm{Chi}^{2}$ ) und machen weniger positive Erfahrungen (41\% gegenüber $62 \% ; \mathrm{p}<0,05 \mathrm{Chi}^{2}$ ) als Frauen, die keine Mutationsträgerinnen sind. Entsprechend würden signifikant weniger Mutationsträgerinnen als Frauen ohne Mutation den Test anderen Frauen empfehlen (55\% gegenüber $70 \% ; \mathrm{p}<0,05 \mathrm{Chi}^{2}$ ), obwohl sich die Mehrheit der Frauen aus beiden Gruppen erneut selbst einem Gentest unterziehen würde. Aufgrund der Ergebnisse erscheint es sinnvoll, Strategien zu entwickeln und den Ratsuchenden an die Hand zu geben, um den Kommunikationsprozeß innerhalb der Familien mit erblichem Brust- und Eierstockkrebs zu erleichtern.

P26

Implantierbare venöse Portkatheter bei Patientinnen mit Mammakarzinom oder gynäkologischen Malignomen: Analyse Sicherheit, Praktikabilität und Einfluss auf die Lebensqualität

Hilde Kreis ${ }^{1}$, Christian R. Löhberg ${ }^{1}$, ${ }^{*}$ Peter A. Fasching ${ }^{1}$, Michael L. Lux ${ }^{1}$, Uwe G. Pöhls' ${ }^{1}$, Werner Lang ${ }^{2}$, Matthias W. Beckmann ${ }^{1}$

${ }^{1}$ Universitätsklinikum Erlangen, Frauenklinik, Erlangen, Deutschland ${ }^{2}$ Chirurgische Klinik, Abteilung für Gefäßchirurgie,

Universitätsklinikum, Erlangen, Deutschland

Zielsetzung: Die Anzahl der Chemotherapien in der gynäkologischen Onkologie nimmt zu, besonders bei Patientinnen mit Mammakarzinom Durch implantierbare venöse Portkatheter (IVP) kann die Applikation der Chemotherapeutika erfolgen und damit die durch periphere Venenapplikation bedingte Langzeitmorbidität der Patientinnen reduziert werden. Ziel dieser Studie ist die Sicherheit und Praktikabilität der IVP's und deren Einfluss auf die Lebensqualität bei onkologischen Patientinnen zu untersuchen. Materialien und Methoden: Vom 01.04.2001 bis 31.07.2003 nahmen 232 onkologische Patientinnen, davon über $80 \%$ mit einem Mammakarzinom, die ein IVP erhalten hatten, an dieser Studie teil. Sie erhielten einen neu entwickelten standardisierten Fragebogen zu IVP, der medizinische Aspekte, Aspekte des täglichen Lebens sowie Aspekte der allgemeinen Zufriedenheit als auch Ängste erfragt. Die Komplikationsrate wurde anhand der Krankenakten erfasst. Ergebnisse: Die Mehrheit der Patientinnen (71\%) ist mit dem IVP ziemlich bzw. sehr zufrieden. Die Armbeweglichkeit ist bei $82 \%$ der Patientinnen überhaupt nicht oder nur ein wenig beeinflusst. Die Mehrheit fühlt sich nicht gestört bei Aktivitäten allgemein (87\%). 62\% der Patientinnen sind in der Lage zu arbeiten, einschließlich der Arbeit zu Hause. Sie können ihre Freizeit genießen (78\%). 30\% der Patientinnen waren mit der Portkatheterpflege außer- 
halb des Klinikums nicht zufrieden. Die Komplikationsrate betrug 6,9\% Schlussfolgerung: IVP sind sicher und effektiv und haben keine relevanten Einwirkungen auf die Lebensqualität. Für die Patientinnen ist der IVP eine gute akzeptierte Methode zur Applikation von toxischen Chemotherapieprotokollen.

\section{P27}

Case-Management in der sektorenübergreifenden Versorgung von Frauen mit Brustkrebs am Beispiel einer Begleitstelle in Augsburg

*Andrea Thorenz, Christa Rottscheidt

beta Institut für sozialmedizinische Forschung und Entwicklung $\mathrm{gGmbH}$

Zielsetzung: Im Disease-Management-Programm Brustkrebs erhält die psychosoziale Versorgung zwar einen hohen Stellenwert zugeschrieben, dies wird allerdings nicht ausreichend umgesetzt. Es fehlt die praktische Verbesserung der psychosozialen Unterstützung auf Patientenseite, die Erarbeitung von individuellen Versorgungsplänen und eine echte sektorenübergreifenden Versorgung der onkologischen Patientinnen. Brustkrebspatientinnen sollen daher neben einer medizinischen auch eine evidenzgestützte und leitlinienbasierte psychosoziale Versorgung erfahren. Diese Versorgung wird im Augsburger mammaNetz derzeit umgesetzt. Das mammaNetz arbeitet nach der Case Management Methode. Materialien und Methoden: Das bundesweit einmalige Projekt wird vom Bayerischen Gesundheitsministerium innerhalb der Gesundheitsinitiative «Bayern aktiv» mit 1,25 Mio Euro bezuschusst. Ein modellhafter PatientinnenPathway ermöglicht der Patientin schon einen Erstkontakt mit der Case Managerin kurz nach der Diagnosestellung bzw. im Gespräch gemeinsam mit der betreuenden Ärztin/Arzt. Die Case Managerin begleitet die Patientin dann über ihre Erstbehandlung hinaus, je nach der Bedürfnislage. Screeninginstrumente in Anlehnung an die ICF-Verfahren der WHO, explizite Interventionsformen und eine Evaluation der Versorgungsergebnisse zählen zu den grundlegenden Case Management Instrumenten, die im Projekt eingesetzt und erprobt werden. Das Projekt wird wissenschaftlich begleitet mit Prof. Dr. Uwe Koch, Prof. Dr. Anita Pfaff und Prof. Dr Axel Tuma sowie der Bayerischen Krebsgesellschaft. Ergebnisse: Der Zwischenbericht der Studie hat ergeben, dass die Angst durch den Einsatz von Case Management reduziert, die Lebensqualität erhöht werden kann und sich ökonomische Einspareffekte zeigen. Zusammenfassung Case Management muss ein integraler Bestandteil von Disease-Management-Programmen werden. Gefordert werden patientenorientierte, qualitätsgesicherte und kosteneffiziente Strategien. Case Management bietet hier einen zukunftsweisenden Ansatz.

\section{P28}

\section{Verlauf nach Mammakarzinom ohne axillären Lymphknotenbefall}

*Arne Willruth, Rainer Callies, Rainer Kimmig UFK Essen, Essen, Deutschland

Der Anteil der Mammakarzinome ohne axilläre Absiedlungen bei Primärdiagnose ist heute größer als in den siebziger und achtziger Jahren. Zwischen 1988 und 1997 wurden in der Universitätsfrauenklinik Essen insgesamt 938 Fälle mit Mammakarzinom primär behandelt. Bei 56\% handelte es sich um pNOpM0 Karzinome.

Ziel dieser retrospektiven Studie ist die Analyse der Überlebensraten und des Follow up bei unilateralen invasiven, nodal negativen Mammakarzinomen bis Januar 2002

Klinische Daten: Tumorgröße: pT1 247 (57,4\%), pT2 170 (39,6\%), pT3 12 (2,8\%) und pT4 $1(0,2 \%)$. Operative Therapie: $57 \%$ BET, $43 \%$ Mastektomie. Die Überlebensraten nach 5 und 10 Jahren für das hier betrachtete Kollektiv liegen bei $90,8 \%$ bzw. $77,7 \%$. Überraschend ergibt die Life Table Analyse bei Untergruppierung nach Tumorgröße keine signifikant unterschiedlichen Überlebenskurven.

Auch wenn man die pT2-pT4 Karzinome zusammenfassend den pT1 Fällen gegenüberstellt ergibt sich kein signifikanter Unterschied. Die kalkulierten Überlebensraten nach 10 Jahren für Frauen mit den primär größeren Karzinomen liegen etwa $6 \%$ niedriger ( $74 \%$ vs. $80 \%$, nicht signifikant).
Die zusätzlich vorgenommene Todesursachenanalyse der pT1 Fälle ergibt 38 Todesfälle im Beobachtungszeitraum. Bei 27 dieser Fälle konnte die Todesursache geklärt werden: 14 verstarben infolge des Mammakarzinom (51\%), 5 infolge eines anderen malignen Tumors und 8 an benignen Erkrankungen. Das hier beobachtete Phänomen des geringen Einflusses der Tumorgröße auf die Prognose ist am ehesten dadurch zu erklären, dass eine artifizielle Gruppenbildung zugrunde liegt. Die größeren Tumoren ohne primären Lymphknotenbefall entsprechen offenbar einer Selektion von weniger aggressiven Tumoren, die nicht proportional der Primärtumormasse metastasieren

\section{Poster: Adjuvante Therapie}

P29

\section{ADEBAR- Studie: Phase III Studie zur Evaluierung der Rolle von Docetaxel in der adjuvanten Therapie von Patientinnen mit Hochrisiko-Mammakarzinom ( $\geq 4$ LK)}

${ }^{*}$ K. Gauger ${ }^{1}$, F. von Bismarck', M. Heinrigs ${ }^{2}$, B. Rack ${ }^{2}$, W. Janni ${ }^{2}$ J. de Waal ${ }^{3}$, D. Steinfeld ${ }^{4}$, D. Augustin ${ }^{5}$, W. Simon ${ }^{6}$, Th. Zwingers ${ }^{7}$, H. Sommer ${ }^{2}$, C. Schindlbeck ${ }^{2}$, K. Friese ${ }^{2}$, N. Harbeck ${ }^{1}$, M. Kiechle ${ }^{1}$ ${ }^{1}$ Frauenklinik der Technischen Universität, München, Deutschland ${ }^{2}$ I. Frauenklinik der Ludwig-Maximilians-Universität, München, Deutschland

${ }^{3}$ Frauenklinik der Kreiskliniken, Dachau, Deutschland

${ }^{4}$ Zentralklinikum, Augsburg, Deutschland

${ }^{5}$ Mammazentrum, Deggendorf, Deutschland

${ }^{6}$ Robert-Bosch Krankenhaus, Stuttgart, Deutschland

${ }^{7}$ Estimate $\mathrm{GmbH}$, Augsburg, Deutschland

Zielsetzung: Aktuelle Daten unterstützen die Hypothese der Überlegenheit von Taxanen in der adjuvanten Therapie des Mammakarzinoms. Dennoch bleiben sowohl die Indikation bei Patientinnen mit mehr als 3 befallenen axillären Lymphknoten, als auch das optimale Therapieregime bisher ungeklärt. Ziel dieser Studie ist es daher, Toxizität und Überlebensraten der Patientinnen gegenüberzustellen, die eine entweder auf Taxanen oder Antrazyklinen basierende Therapie erhalten. Materialien und Methoden: Es handelt sich um eine multizentrische, prospektiv randomisierte Phase III Studie in der Adjuvanz mit 4 Zyklen Epirubicin $90 \mathrm{mg} / \mathrm{m}^{2}$ und Cyclophosphamid $600 \mathrm{mg} / \mathrm{m}^{2} \mathrm{q} 3 \mathrm{w}$ gefolgt von 4 Zyklen Docetaxel $100 \mathrm{mg} / \mathrm{m}^{2}$ q3w (E g $_{90}$ C-Doc) versus 6 Zyklen Epirubicin $60 \mathrm{mg} / \mathrm{m}^{2}$ i.v. d 1+8, 5-Fluorouracil $500 \mathrm{mg} / \mathrm{m}^{2}$ i.v. $\mathrm{d} 1+8$ und Cyclophosphamid $75 \mathrm{mg} / \mathrm{m}^{2}$ p.o. $\mathrm{d} 1-14$, q $4 \mathrm{w}$. Patientinnen mit positivem Hormonrezeptorstatus erhalten anschließend eine endokrine Therapie, zudem ist bei gegebener Indikation eine adjuvante Strahlentherapie obligat. Ergebnisse: 174 aktive Zentren randomisierten bisher 958 Patientinnen. Bei $60 \%$ liegen 4-9, bei 40\% mehr als 10 axilläre Lymphknotenmetastasen vor. Zwei Drittel der Patientinnen sind Hormonrezeptor-positiv. In $82 \%$ der Fälle wird die Strahlentherapie der Chemotherapie nachgeschaltet. Serious adverse events traten in 2,8\% der Chemotherapiezyklen auf. Im wissenschaftlichen Begleitprogramm der Studie wird der prädiktive Wert von uPA/PAI-1 des Primärtumors und disseminierter Tumorzellen im Knochenmark bei Primärdiagnose und nach Abschluss der Chemotherapie geprüft. Zusammenfassung: Die exzellente, stetig zunehmende Rekrutierung und geringe Zahl an Komplikationen bestätigen die gute Durchführbarkeit der ADEBAR-Studie. Sie wird einen wichtigen Beitrag zur Klärung der Rolle eines sequentiellen Taxanschemas beim Mammakarzinom mit ausgedehnter Lymphknotenmetastasierung leisten.

\section{P30}

\section{Biologische Wirkung eines niederenergetischen}

\section{Röntgenstrahlers bei intraoperativer Teilbrustbestrahlung}

${ }^{*}$ Carsten Herskind, Volker Steil, Uta Kraus-Tiefenbacher, Frederik Wenz Abt. Strahlentherapie, Universitätsklinikum Mannheim, Mannheim, Deutschland

Zielsetzung: Intraoperative Teilbrustbestrahlung wird derzeit in einer randomisierten klinischen Studie bei Brustkrebspatientinnen untersucht. Die Verwendung niederenergetischer Röntgenstrahlung (30-50 kV) ermöglicht die Applikation hoher lokaler Strahlendosen bei unproblematischem 
Strahlenschutz. Diese Strahlenmodalität unterscheidet sich von konventioneller externer Bestrahlung durch die erhöhte relative biologische Wirksamkeit (RBW) niederenergetischer Photonen, steile Dosisgradienten und längere Bestrahlungszeiten, die eine Reparatur während der Einzeitbestrahlung ermöglichen. Ziel war es, die zu erwartenden biologischen Effekte im Normal- und Tumorgewebe als Funktion der Tiefe im Gewebe zu berechnen. Materialien und Methoden: Sphärische Applikatoren (2-5 $\mathrm{cm}$ Diameter) für Tumorbettbestrahlung mit dem miniaturisierten Beschleuniger Intrabeam (Carl Zeiss, Oberkochen) ergeben nahezu isozentrische Dosisverteilungverteilungen. Die RBW wurde mit dem linear-quadratischen Formalismus unter Berücksichtigung der Reparatur während der Bestrahlung berechnet. Ergebnisse: Für 50 kV Strahlung und eine Dosis von $20 \mathrm{~Gy}$ an der Applikatoroberfläche. nahm die RBW für spätreagierendes Normalgewebe mit zunehmender Tiefe zu $(0,9-1,1$ an der Oberfläche, 1,5 in 13-21 mm Tiefe). Das berechnete 50\% Risikoniveau für Pneumonitis bzw. subkutane Fibrose in der umschlossenen Volumeneinheit wurde in $6-11 \mathrm{~mm}$ Tiefe bzw. 3-6 mm Tiefe erreicht und nahm mit zunehmender Tiefe schnell ab. Die Tumorkontrolle wurde nah an der Applikatoroberfläche höher als für konventionelle Bestrahlung geschätz und nahm mit zunehmender Tiefe ab. Zusammenfassung: Der Applikatordiameter ist ein bedeutender Einflussparameter der biologischen Wirkung. Für Tumorbettbestrahlung bis $10 \mathrm{~mm}$ Tiefe sind Spätreaktionen im Normalgewebe nur in diesem Bereich zu erwarten. Der Volumeneffekt bewirkt aber wahrscheinlich eine weitere Reduktion des klinisch betroffenen Volumens. Modellberechnungen lassen vermuten, dass ein «Äquivalenzradius» für das Rezidivrisiko im Vergleich zu konventioneller Bestrahlung existiert.

\section{P31}

\section{2-Methoxyestradiol hat in Kombination mit Chemotherapeutika eine additiv antiproliferative Wirkung auf menschliche Ovarialkarzinomzellen}

*Alfred O. Mueck, Harald Seeger, Jens Huober, Diethelm Wallwiener Universitäts-Frauenklinik, Tübingen, Deutschland

Zielsetzung: 2-Methoxyestradiol (2ME), ein endogener Estradiolmetabolit, zeigt starke antiproliferative und antiangiogene Eigenschaften bei verschiedenen Krebszellarten. Wir haben untersucht, ob 2ME in vitro in Kombination mit verschiedenen Chemotherapeutika eine additive antiproliferative Eigenschaft bei menschlichen Ovarialkarzinomzellen aufweist. Materialien und Methoden: Als Zellmodel wurden zwei verschiedene menschliche Ovarialkarzinomzellen verwendet. Die Zell-Linien wurden mit einer konstanten Konzentration von $2 \mathrm{ME}(25 \mu \mathrm{M})$ und verschiedenen Konzentrationen $(0,8-25 \mu \mathrm{M})$ der Chemotherapeutika Epirubicin, Doxorubicin, Paclitaxel, Docetaxel, Carboplatin, Vinorelbin, 5-Fluorouracil und Mafosfamid inkubiert. Die Proliferation wurde nach vier Tagen mittels ATP-Chemosensitivitätstest gemessen. Hierbei wird die Lichtemission von Luziferin unter Anwesenheit von ATP und Luziferase quantifiziert. Ergebnisse: In beiden Zell-Linien fand sich ein signifikanter additiver antiproliferativer Effekt für die Kombinationen aus 2ME und Epirubicin und Carboplatin, die vor allem im unteren Konzentrationsbereich der Zytostatika zu beobachten war. Die Wirkung der Zytostatika konnte dabei von $20-30 \%$ auf $40-60 \%$ gesteigert werden. In allen anderen Kombinationen war der Effekt der Zytostatika dominant. Zusammenfassung: 2-Methoxyestradiol wurde bisher in klinischen Studien bei metastasiertem Brust- und Prostatakrebs getestet. Die Tolerabilität von $2 \mathrm{ME}$ erwies sich dabei als ausgezeichnet. Entsprechend der vorliegenden Ergebnisse könnte 2ME auch als eine adjuvante Therapiemöglichkeit zusammen mit bestimmten Zytostatika in Frage kommen. Derzeit gibt es dazu allerdings noch keine klinischen Untersuchungen.

\section{P32}

Salvage-Therapie mit Bendamustin bei Patientinnen mit intensiv vorbehandeltem, metastasierten Mammakarzinom: Resultate einer Phase-II-Studie

\section{*Ursula Reichmann, Jens Huober, Carsten Bokemeyer,}

Diethelm Wallwiener, Michael Bamberg

Abteilung für Radioonkologie Universität Tübingen, Deutschland

Wir führten eine multiinstitutionale Phase-II-Studie mit Bendamustin einem Stickstofflostderivat aus der Gruppe der bifunktionellen Alkylanzien, bei ausgedehnt vorbehandelten Patientinnen mit metastasiertem Mammakarzinom durch

36 Patientinnen wurden mit Bendamustin $120 \mathrm{mg} / \mathrm{m}^{2}$ i.v. Tag $1+2$ alle 4 Wochen behandelt. Prophylaktisch wurden Serotoninantagonisten gegeben. Insgesamt wurden 150 Kurse verabreicht, im Mittel 4 pro Patientin (1-8). Das mediane Alter betrug 60 Jahre (37-75), die Patientinnen hatten im Mittel 3 Vortherapien, davon 25/36 mit Anthrazyklinen und Taxanen $(69,4 \%)$. 6/36 Patientinnen (16,7\%) hatten eine Metastasenlokalisation, $13 / 36(36,1 \%)$ hatten 2, 9/36 (25,0\%) 3 und 8/36 (22,2\%) 4 wie Knochen, Gehirn, Lunge, Lymphknoten, Pleura oder Leber.

Die Toxizität war relativ mild, $\mathrm{III}^{\circ} / \mathrm{IV}^{\circ}$ Toxizitäten traten selten auf. Nur 1 Patientin entwickelte eine $\mathrm{IV}^{\circ}$ Thrombopenie (2,8\%), 2 Patientinnen eine III $^{\circ}$ Thrombopenie (5,6\%), 7 Patientinnen eine III $^{\circ}$ Leukopenie $(19.5 \%)$ und 1 Patientin eine $\mathrm{III}^{\circ}$ Übelkeit $(2,8 \%)$. $\mathrm{I}^{\circ} / \mathrm{II}^{\circ}$ Toxizitäten traten folgende auf: Übelkeit 20/36 (55,6\%), Leukopenie 20/36 (55,6\%), Thrombopenie $10 / 36(27,8 \%)$, Infektionen $15 / 36$ (41,7\%), Fieber 12/36 (33,4\%), Mukositis 7/36 (19,5\%), Diarrhoe 7/36 (19,5\%), Dyspnoe 12/36 (33,4\%), Neurotoxizität $7 / 36(19,5 \%)$, alle anderen waren unter $10 \%$. Haarausfall nur $\mathrm{I}^{\circ}$

Bei 35 auswertbaren Patientinnen sahen wir keine Vollremission, 8 Partialremissionen, entsprechend einer Ansprechrate von 22,2\%, no change (NC) bei 12/36 Patientinnen (34,6\%) und eine Progression unter Therapie bei $15 / 36$ Patientinnen (41,7\%).

Die Therapie mit Bendamustin wird von Patientinnen mit metastasiertem Mammakarzinom gut vertragen und ist auch nach ausgedehnter Vorbehandlung effektiv. Weitere Studien mit anderen Applikationsschemata und in Kombination mit anderen Zytostatika sollten folgen.

P33

\section{Bi-weekly PEGyliertes liposomales Doxorubicin in der} Behandlung des fortgeschrittenen Mammakarzinoms. Eine Phase II Studie

${ }^{*}$ D. Roßner ${ }^{1}$, Ch. Schaff 1 , A. Ay ${ }^{1}$, W. Lersch ${ }^{2} ;$ F. Rinnau ${ }^{1} ;$ B. Hollwitz ${ }^{1}$; H. Kühnle ${ }^{1} ;$ H. J. Lück ${ }^{1}$

${ }^{1}$ Gyn. Onkologie/Brustzentrum MH, Hannover, Deutschland

${ }^{2}$ Essex Pharma, München, Deutschland

Peg-Liposomales Doxorubicin stellt eine Alternative zu einer Behandlung mit freiem Doxorubicin dar. In randomisierte Studien beim Mammakarzinom konnte die Äquieffektivetät nachgewiesen werden. Die beiden Formen des Doxorubicin unterscheiden sich bezüglich seines Nebenwirkungsspektrums grundsätzlich. Beim freien Doxorubicin stehen Myelosuppression, Stomatitis und cardiologische Veränderungen im Vordergrund. Bei der liposomalen Form sind diese Nebenwirkungen deutlich weniger relevant. Allerdings stellen Hautveränderungen ein die Patientinnen stark belastendes Problem dar, insbesondere wenn die Therapie über einen längeren Zeitraum durchgeführt wird. Untersuchungen beim Kaposi-Sarkom weisen auf eine bessere Verträglichkeit einer zweiwöchigen Gabe hin. Ziel dieser Untersuchung war es diese Applikationsform beim weit fortgeschrittenen Mammakarzinom zu überprüfen. Hierzu wurde 25 Patientinnen mit $20 \mathrm{mg} / \mathrm{m}^{2}$ Peg-Liposomalem Doxorubicin (Caelyx) alle zwei Wochen behandelt. Nach der dritten Caelyx-Gabe erfolgte eine 4 wöchige Pause. Bei Patientinnen ohne Progressionszeichen wurde ein zweiter Block von drei Caelyx-Gaben durchgeführt. Therapie erfolgte bis zur Progression. Die med. Zahl der Vortherapien war 5, und 12 v. 25 Pat. War bereits mit Anthrazyklinen behandelt worden, und 18 von 25 mit Taxanen. Toxizität: Es traten keine hämatologischen Grad III/IV Toxizitäten auf. Ebenso konnten keine nicht-hämatologischen Grad III/IV Toxizitäten beobachtet werden. Dies gilt insbesondere für die Hauttoxizität. Effektivität: Die Ansprechrate war dem mehrfach chemo- 
therapeutisch vorbehandelten Patientenkollektiv mit 3 PR und 3 SD akzeptabel. Die med. Zeit bis zur erneuten Progression betrug bei den Patientinnen mit PR 14 Monate und bei den Patientinnen mit einer SD 11 Monate. Schlussfolgerung: Pegyliertes Doxorubicin ist eine wirksame und nebenwirkungsarme Behandlungsoption beim vorbehandelten Mammakarzinom. Die zweiwöchige Applikationsform sollte in einer randomisierten Studie mit der 4-wöchigen Gabe verglichen werden.

\section{P34}

\section{Phase II trial of biweekly pegylated Liposomal Doxorubicin (Caelyx) in heavily pretreated, metastatic breast cancer}

*Peter Schmid, Carsten-Oliver Schulz, Annette Dieing, Anne Roever, Bernd Flath, Orhan Sezer, Kurt Possinger

Medizinische Klinik mit Schwerpunkt Onkologie und Hämatologie,

Charite Campus Mitte, Humboldt Universität Berlin, Berlin, Germany

Introduction: Pegylated liposomal doxorubicin (Caelyx) is as effective a conventional doxorubicin but provides a lower risk of cardiotoxicity. Caelyx is commonly administered in 4 weeks intervals. This phase II was initiated to evaluate the efficacy and tolerability of a biweekly schedule. Patients and Methods: Patients with metastatic breast cancer who had received at least 2 prior chemotherapy regimens for metastatic disease received Caelyx $\left(25 \mathrm{mg} / \mathrm{m}^{2}\right)$ in 2 week intervals for up to 12 courses Pretreatment with anthracyclines was allowed. Results: 24 heavily pretreated patients (visceral metastases $88 \%$; median number of metastatic site 3 ; median number of prior chemotherapies 4 ; prior anthracyclines $88 \%$ taxanes $83 \%$, vinorelbine $75 \%$, capecitabine $46 \%$ ) received a total of 109 cycles. Treatment was generally well tolerated. Myelosuppression was low, with no grade 3/4 leukopenia, neutropenia or thrombocytopenia. Most common toxicities were nausea, alopecia, asthenia and hand-foot syndrome. Grade 3 toxicities were reported with hand-foot syndrome, infection, dyspnea and cardiotoxicity. One patient had an anaphylactic reaction. No other grade 4 toxicities were observed. Nineteen patients are assessable for response. One patient achieved a partial response and 3 patients had stable disease for more than 6 months accounting for a clinical benefit of $21 \%$. Three of these 4 patients had received prior anthracycline therapy for metastatic disease. Median duration of clinical benefit was 9.3 months Median TTP and overall survival were 7 weeks and 9.6 months, respectively. Conclusions: Biweekly Caelyx is a safe schedule with a favorable toxicity profile. Efficacy was moderate in anthracycline pretreated patients.

P35

Randomized Trial Comparing Up Front Tandem High-Dose Chemotherapy and Standard Chemotherapy with Doxorubicin and Paclitaxel in Metastatic Breast Cancer

*Peter Schmid ${ }^{1}$, Walter Schippinger ${ }^{2}$, Thorsten Nitsch ${ }^{3}$, Gerdt Huebner ${ }^{4}$, Rolf Kreienberg ${ }^{5}$, Wolfgang Schultze ${ }^{6}$, Hubert Hausmaninger ${ }^{7}$, Manfred Wischnewsky ${ }^{8}$, Kurt Possinger ${ }^{1}$

${ }^{1}$ Medizinische Klinik mit Schwerpunkt Onkologie und Hämatologie, Charité Campus Mitte, Humboldt Universität Berlin, Berlin,

Deutschland

${ }^{2}$ Abteilung für Onkologie und Hämatologie, Universitätsklinikum, Graz, Österreich

${ }^{3}$ Onkologie und Hämatologie, Franziskus Hospital, Bielefeld,

Deutschland

${ }^{4}$ Onkologie und Hämatologie, Westpfalz-Klinikum, Kaiserslautern, Deutschland

${ }^{5}$ Universitätsfrauenklinik, UIm, Deutschland

${ }^{6}$ Humaine Klinikum, Bad Saarow, Deutschland

${ }^{7}$ Onkologie und Hämatologie, Landesklinik, Salzburg, Österreich

${ }^{8}$ Department for Intelligent Systems, University of Bremen,

Bremen, Germany

Patients and Methods: Patients without prior chemotherapy for metastatic disease were randomly assigned to standard combination therapy with doxorubicin and paclitaxel (AT) or double HDCT with cyclophosphamide, mitoxantrone and etoposide followed by peripheral blood stem cell transplantation. HDCT was repeated after 6 weeks. Patients were stratified by menopausal and hormone receptor status. The primary objective was to compare complete response (CR) rates. Results: A total of 93 patients were enrolled in the trial. Intent-to treat $\mathrm{CR}$ rates for patients randomised to HDCT and AT were $12.5 \%$ and $11.1 \%$, respectively $(\mathrm{p}=0.84)$. Objective response rates were $66.7 \%$ for patients in the high-dose group and $64.4 \%$ for patients in the AT-arm $(\mathrm{p}=0.82)$. In an intent-to treat analysis, there were no significant differences between the two treatments in median time to progression (HDCT 11.1 months, AT 10.6 months; $\mathrm{p}=0.67$ ), duration of response (HDCT 13.9 months, AT 14.3 months; $\mathrm{p}=0.98$ ), and overall survival (HDCT 26.9 months, AT 23.4 months; $p=0.60$ ). HDCT was associated with significantly more myelosuppression, infection, diarrhea, stomatitis, nausea and vomiting, whereas patients treated with AT developed more neurotoxicity. Conclusion: This trial failed to show a benefit of up front tandem HDCT over standard combination therapy. HDCT was associated with more acute adverse effects.

\section{Poster: Ästhetische Mamma-Chirurgie \\ P36 \\ Plastinationshistologische Untersuchungen zur oberflächlichen Brustfascie}

${ }^{*}$ B. Feit' ${ }^{1}$, L. Busch ${ }^{1}$, J. Hoch ${ }^{2}$

${ }^{1}$ Institut für Anatomie der Medizinischen Universität zu Lübeck, Lübeck, Deutschland

${ }^{2}$ Klinik für Hand-, Brust- und Plastische Chirurgie, Klinikum Neustadt in Holstein, Neustadt in Holstein, Deutschland

Im Streben nach weniger radikalen und gewebeschonenden Operationstechniken an der weiblichen Brust gewinnt die Suche nach anatomischen Strukturen Bedeutung, die ein brusterhaltendes Vorgehen im Sinne einer subkutanen Mastektomie ermöglichen, ohne das Risiko von Lokalrezidiven wesentlich zu erhöhen. Bei dieser Struktur könnte es sich um eine bereits klinisch diskutierte Schicht, die Lamina superficialis der Fascia superficialis handeln, deren dreidimensionaler Aufbau bislang histologisch noch nicht dargestellt worden ist. Material und Methoden: Insgesamt wurden 6 Mammae von drei Frauen im Alter von 30, 78 und 97 Jahren untersucht. Die Aufarbeitung erfolgte nach dem plastinationshistologischen Verfahren von Dr. von Hagens. Hierbei wird das Körperfett gegen ein Epoxidharzgemisch ausgetauscht. Die Präparate gewährleisten dabei aufgrund der unveränderten Topographie verbindliche Aussagen über die räumliche Ausdehnung der zu untersuchenden Strukturen. Ergebnisse /Zusammenfassung: Die Lamina superficialis (LS) der Fascia superficialis wurde in allen Präparaten als konstante anatomische Struktur der gesamten Brust nachgewiesen und läuft im Bereich der unteren Umschlagfalte mit der Fascia superficialis und der Fascia profunda zusammen, die sich dann in der Fascia Scarpae auf der vorderen Brustwand fortsetzt. Ihre Ausprägung sowie ihr Abstand zur Haut wechselt sehr und scheint abhängig vom Alter und Ernährungszustand zu sein. Ferner wird sie sowohl von Gefäßen und Lymphbahnen, als auch teilweise von den Cooper'schen Ligamenten durchbrochen und ist an manchen Stellen insgesamt so dünn, daß sie nur histologisch nachweisbar ist. Als Präparationsschicht bei einer subkutanen Mastektomie unter Einhaltung onkologischer Kautelen oder bei formverändernden Eingriffen der Brust kommt sie dementsprechend nur partiell und je nach individueller Konstitution zur Anwendung.

P37

\section{Therapie der Gynäkomastie}

*Christian Roessing, Siegfried Grandel, Anette Görg Klinik für Plastische Chirurgie, Hand- und Wiederherstellungschirurgie, Luisenhospital Aachen, Aachen, Deutschland

Das operative Vorgehen und die kosmetischen Resultate der Therapie der Gynäkomastie mit Liposuction versus Mastektomie in unserem Patientengut wird vorgestellt.

Ursachen für Rezidive, Häufigkeit der Nachkorrekturen wie Straffung des Hautmantels nach Liposuction und Verkleinerung des MammillenAreolakomplexes mit zirkulärer Straffung werden diskutiert. 
P38

\section{Mammaaugmentation: Pro und Contra der verschiedenen Augmentationstechniken}

*Andrea Schulz, I. Herren, Andrea Arens, Jutta Liebau Fachklinik Hornheide, Abteilung für Plastische Chirurgie, Münster, Deutschland

Die optimale Positionierung eines Implantates zur Augmentation ist eine große Herausforderung in der Behandlung von Mammahypotrophien und Involutionshypoplasien.

Im Zeitraum von 2000 bis 2004 wurden über 150 Patienten mit Mammahypotrophien und Involutionshypoplasien mit und ohne Ptosis der Brust an der Fachklinik Hornheide behandelt. Die Entscheidung, ob ein Implantat sub.- oder epipectoral zu positionieren ist hängt im Wesentlichen von den anatomischen Voraussetzungen, der Hautbeschaffenheit und eventuell bestehender Voroperationen ab. Einzubeziehen in die Entscheidungsfindung ist der mögliche Zugangsweg bei der jeweiligen Implantatlage. Perimammilärer, submammärer oder axillärer Zugang bieten unterschiedliche Vor- und Nachteile die berücksichtigt werden sollten. Be einer Ptosis mammae ist die Augmentation gelegentlich mit einer Hautmantelstraffung zu kombinieren. Wir berichten über Indikationsstellun gen der jeweiligen Techniken unter Berücksichtigung sehr positiver Erfahrungen mit der submuskulären Augmentation über einen submammären Zugangsweg. Zur Augmentation wurden ausschließlich silikongefüllte, Mikropolyurethanschaum-beschichtete Mammaimplantate der Firma Silimed verwendet. Als perioperatives Procedere kamen eine intravenöse Antibiose, Redondrainagen und ein Stütz BH mit belt zur Anwendung

\section{P39}

\section{Modifikation der Mammareduktionsplastik nach Léjour: Cut as you go}

*Nestor Torio-Padron, Gunter Felmerer, Arash Momeni, Matthias Föhn, Jörg Borges, G. Björn Stark

Abteilung für Plastische und Handchirurgie, Universitätsklinikum Freiburg, Freiburg, Deutschland

Zielsetzung: Prinzip der Technik nach Léjour ist eine schlüsselförmige präoperative Markierung mit Festlegung der Hautresektion (Léjour 1990) Demgegenüber beruht die bewährte Reduktionsmethode nach Höhler (1961) auf einer T-förmigen Resektion (mit dem Nachteil einer inframammären queren Narbe) und der Formung der Brust durch die Anpassung des Hautmantels (mit dem Nachteil der späteren «drop out»oder «star gaze»-Deformität). Die von uns verwendete Technik kombinier die Vorteile unter Vermeidung der Nachteile beider Techniken. Materialen und Methode: Präoperativ wird nur die geplante Position des Areolenoberrandes markiert. Die Resektion sowie Formung der Brust erfolgt im Wesentlichen nach Léjour, wobei die endgültige Hautresektion während der Operation («cut as you go») und die Umschneidung des neuen Areolenrandes intraoperativ, allerdings unter Nutzung der Schrumpfung der subdermal mobilisierten Haut ohne horizontale Schnittführung erfolgt Zusammenfassung: Die Kombination der Techniken erlaubt eine große Variabilität in der Anwendung der entsprechenden Operationsmethode für alle Brustgrößen und vermeidet präoperative Planungsfehler.

\section{Poster: Diagnostik}

P40

\section{Die Brustselbstuntersuchung nach der MammaCare-Methode} als Bestandteil der Brustkrebs-Früherkennung

${ }^{*}$ Almut Artmann 1, 2, Dorothy Berthold ${ }^{1}$, Michaela Heyne 1 ,

Barabara Rüssmann ${ }^{1}$, Marion Kiechle ${ }^{1}$, Nadia Harbeck ${ }^{1}$

${ }^{1}$ Frauenklinik rechts der Isar, München, Deutschland

${ }^{2}$ Mammazentrum Klinikum rechts der Isar, München, Deutschland

Zielsetzung: $80 \%$ aller mit einem Tastbefund assoziierten Mamma-Carcinome werden von den Frauen selbst erhoben. Nur max $40 \%$ aller Frauen führen eine Brustselbstuntersuchung (BSU) durch. Der Hauptgrund ist die Angst eventuell Tastbefunde nicht zuordnen zu können. Jedoch liegt gerade in der Früherkennung von Mamma-Carcinomen und einem kurzen Zeitintervall bis zur Abklärung die Chance für ein längeres rezidivfreies Überleben und eine bessere Lebensqualität. Gemäss der S3-Leitlinie ist die BSU ein Bestandteil der Brustkrebsfrüherkennung. Materialien und Methoden: Die systematische BSU nach der MammaCare-Methode, die in unserem Zentrum an interessierte Frauen und Multiplikatorinnen vermittelt wird, ermöglicht an orginalgetreuen Silikonmodellen das systematische und differenzierte Ertasten verschiedener Knotenqualitäten auf unterschiedlichem Höhenniveau innerhalb der Modelle. Zielgruppe sind alle Frauen sowie Tumornachsorge-Patientinnen (Mammakarzinom). Unter Supervision wird das Erlernte auf die eigene Brust übertragen. Fragebögen zur Kursevaluation werden ausgeteilt. Alle bisher veröffentlichten Studien zur BSU auch hinsichtlich der Mortalitätssenkung beim Mamma-Carcinom basieren nicht auf einer derartigen Systematik. Ergebnisse: Nach 6-monatiger Anwendung hat sich bei den Teilnehmerinnen die Angst eine BSU durchzuführen deutlich reduziert. $3 \mathrm{~mm}$ große Knötchen werden immer getastet. Unter wissenschaftlicher Begleitung erfolgt eine Supervision nach einem definierten Zeitintervall mit Kontrolle der Anwendung, apparativer Befundkorrelation sowie der Erfassung der Motivation und aktiven Beteiligung an der Brustkrebstfrüherkennung. Zusammenfassung: Die MammaCare Methode vermittelt mit Hilfe von Silikonmodellen eine strukturierte BSU Technik. Diese Methode schärft das Bewusstsein der Frauen und motiviert, sich aktiv an Brustkrebsfrüherkennung zu beteiligen. Die Anbindung des «MammaCare»-Zentrums an eine Universitätsfrauenklinik garantiert eine hohe Akzeptanz des Mammakarzinom-Screenings. Unsere aktuellen evaluierten Daten werden im Rahmen des Kongresses vorgestellt.

P41

Ein Leistungsvergleich zwischen einem Film-Folien-System und einem Speicherfoliensystem für die Mammographie

${ }^{*}$ C. Blendl ${ }^{1}$, T. Krusche ${ }^{2}$, J. Spielmann ${ }^{1}$

${ }^{1}$ Fachhochschule Köln, Köln, Deutschland

${ }^{2}$ Fuji Foto Film Neuss, Neuss, Deutschland

Zielsetzung: Die Wiedergabe eines Prüfkörpers mit geringer Modulation und unterschiedlichen Ortsfrequenzen sollte bei einem Film-Folien-System (SC 25) und bei einem Speicherfoliensystem getestet werden. Materialien und Methoden: Prüfkörper-Aufnahmen von Linienrastern (Silber mit einer Strahlenmodulation von 3,1\% und $7 \mathrm{~mm}$ - Gold mit 12,8\%) wurden mit einer Mammographie-Röntgeneinrichtung (GE), Typ DMR mit $24 \mathrm{kV}, \mathrm{Mo} / \mathrm{Mo}-\mathrm{Technik}$ und unterschiedlichen mAs-Werten erstellt. Die Dichte des Films lag zwischen 2,5>D>0,5; FFS: Fuji AD-M / AD Mammo Fine. Mit denselben mAs-Werten wurden Speicherfolien exponiert: Typ HR-BD. Auslesung via FCR 5000 MR mit 12Bit-Tiefe und einem Laserspot von $50 \mathrm{~mm}$ Durchmesser. Die Digitalisierung der Röntgenbilder erfolgte mit einem Trommelscanner Primescan D7100. Von allen Rasterabbildungen wurde das Kontrast-Rausch-Verhältnis gebildet: Ergebnisse: Goldraster: Bei einer Dichte von zirka 1,6 löst das analoge System höhere Ortsfrequenzen auf. Bei höheren Dichten $(\mathrm{D}>2)$ fällt die Ortsauflösung unter den Wert des digitalen Systems, dessen Ortsauflösung über den gesamten Expositionsbereich bei $8 \mathrm{LP} / \mathrm{mm}$ liegt. Silberraster: Die Erkennbarkeit endet rauschbegrenzt etwa bei $5 \mathrm{LP} / \mathrm{mm}$. Zusammenfassung: Die Leistung des analogen Systems wird durch seine begrenzte Dynamik limitiert; die des digitalen Systems durch die Abtastfrequenz. Das Verhältnis der CNR2-Werte bei gleicher Ortsfrequenz gibt an, um wie viel Prozent das jeweilige Systems in Abhängigkeit von der Dosis (mAs) besser oder schlechter ist (siehe Abbildung). Bei geringer Strahlenmodulation (3,5\%) ist das digitale Systems bei Werten kleiner als 2,5 LP/mm besser (Werte größer 100\%); bei größeren mAs-Werten gilt dies auch für höhere Ortsfrequenzen. 
P42

\section{Granularzelltumor der Brust}

${ }^{*}$ Katja Bobermien ${ }^{1}$, Ralf Ohlinger ${ }^{1}$, Detlef Arndt ${ }^{1}$, Günter Schwesinger ${ }^{2}$, Günther Köhler ${ }^{1}$

${ }^{1}$ Klinik und Poliklinik für Gynäkologie und Geburtshilfe, Greifswald, Deutschland

${ }^{2}$ Institut für Pathologie

Der Granularzelltumor, synonym als granuläres Neurom oder auch Granularzellmyoblastom bezeichnet, stellt eine seltene zumeist benigne Neoplasie dar, ausgehend von den Schwann-Zellen peripherer Nerven. In 5-6\% der Fälle tritt der Granularzelltumor im Bereich der Brust auf und kann dort sowohl klinisch als auch mammo- und sonographisch das Bild eines invasiven Mammakarzinoms imitieren. Wir berichten über eine $52-$ jährige Patientin, die im Rahmen der Selbstuntersuchung eine derbe Resistenz im sternokostalen Bereich der linken Brust tastete. Das suspekte Areal ließ mammo- und sonographisch sowie im MRT am ehesten an ein Mammakarzinom denken. Die darauf folgende diagnostische Exstirpation erfolgte weit im Gesunden und ergab oben genannten histologischen Befund. Zur Diskussion stehen das pathologische und klinische Erscheinungsbild, Prognose und Therapie dieses seltenen Krankheitsbildes.

\section{P43}

\section{DD der Anisomastie in der Adoleszenz}

*Karin Bock ${ }^{1}$, Anja Weidner ${ }^{1}$, Corinna Heitmann ${ }^{1}$, Annette Ramaswamy ${ }^{2}$, Peyman Hadji ${ }^{1}$, Volker F. Duda ${ }^{1}$, Uwe Wagner ${ }^{1}$ ${ }^{1}$ Klinik für Gynäkologie, Gynäkologische Endokrinologie und Onkologie der Philipps-Univ. Marburg, Marburg, Deutschland ${ }^{2}$ Institut für Pathologie der Philipps-Universität Marburg, Marburg Deutschland

Zielsetzung: Während insbesondere eine peripubertäre Mammaasymmetrie in begrenztem Umfang als physiologisch anzusehen ist, bedarf die postpubertär persisitierende Anisomastie auch wegen häufig resulierender, psychischer Probleme der differentialdiagnostischen Abklärung. Die bildgebende Diagnostik sollte ohne Belastung mit ionisierenden Strahlen erfolgen. Wie häufig ist die Diagnose einer idiopathischen Anisomastie zu stellen? Welchen Stellenwert besitzt die Mammasonographie in der Differentialdiagnose juveniler Mammaasymmetrien? Materialien und Methoden: Aus dem Kollektiv einer Spezialsprechstunde für Kinder- und Jugendgynäkologie mit senologischem Schwerpunkt konnten zwischen 2002 und 2004 insgesamt 13 Patientinnen (Alter 12-17 Jahre) mit ausgeprägter Anisomastie identifiziert werden. Die standardisierte Diagnostik bestand aus der körperlichen Untersuchung mit Bestimmung der pubertären Entwicklung anhand der Stadieneinteilung nach Tanner, der klinischen Untersuchung sowie der Mamma- und Thoraxwand-Sonographie (7,5-13 $\mathrm{MHz}$ ). In ausgesuchten Fällen erfolgte eine weitere Abklärung durch Inanspruchnahme zusätzlicher apparativer Diagnostik, minimal invasive Mammabiopsie oder Karyotypisierung. Ergebnisse: 6 partielle Polandsyndrome unterschiedlichen Ausprägungsgrades; 2 ausgeprägte unilaterale juvenile Papillomatosen (histologisch gesichert); 2 einfache idiopathische Anisomastien; 1 idiopathische Anisomastie mit Hyperthelorismus (Karyotyp oB); 1 intramammärer akzessorischer Drüsenkörper: 1 Anisomastie bei einseitig betonter, tubulärer Mamma. Zusammenfassung: In 10 von 13 Fällen ließ sich eine eindeutige Pathologie (Poland, Papillomatose, akzessorischer Drüsenkörper, tubuläre Mamma) nachweisen, nur in 3 von 13 Fällen handelte es sich tatsächlich um eine idiopathische Anisomastie. Die Mammasonographie erlaubte eine zeitnahe, klare Diagnosestellung und ermöglicht eine perspektivische Therapieplanung bei dadurch verringerter psychischer Morbidität der betroffenen Heranwachsenden.
P44

Dosisreduktion in der Film-Folien-Mammographie Technische und klinische Studien zur neuen Film-FolienKombination Min-R EV von Kodak

*Markus Borowski, Hans Junkermann

Mammographie Screening Bremen, Bremen, Deutschland

Zielsetzung: In einer Zeit, in der immer mehr auch die Mammographie mit digitalen Bildempfängern durchgeführt wird, bringt Kodak ein grundlegend verändertes Film-Folien-System, Min-R EV, auf den Markt. Ziel unserer Untersuchungen war es, die Eigenschaften des neuen Systems auf technischer Seite und im klinischen Einsatz zu bestimmen. Materialien und Methoden: Die technischen Parameter des neuen Systems wurden in Phantomstudien bestimmt und mit denen der Vorgänger-Kombinationen Min-R 2000/ Min-R 2190 bzw. Min-R 2000/ Min-R 2250 verglichen. Die diagnostische Qualität der Film-Folien-Kombination wurde in einer klinischen Studie von 100 Screeningfällen bestimmt. Der Dosisbedarf je Aufnahme wird aktuell im Rahmen einer Studie bestimmt. Ergebnisse: Die maximale Schwärzung des neuen Films ( 4,9 OD) ist gegenüber dem Min-R 2000-Film ( 4,5 OD) leicht erhöht. Der Filmgradient (Mgrad $>4,1$ ) ist viel steiler als beim Min-R 2000. Das Gesamtsystem aus Film und Folie, hat im gesamten Belichtungsbereich ein gegenüber den Vorläufern erhöhten Gradienten. Der diagnostisch nutzbare Bereich wurde auf optische Dichten oberhalb von 3,5 OD ausgedehnt. Die Ortsauflösung wurde leicht erhöht. Subjektive Studien der Bildqualität unter zu Hilfenahme des CDMAM-Phantoms ergaben einen um $10 \%$ verbesserten IQF-Wert. In der klinischen Routine wurde die Film-Folien-Kombination erfolgreich unter Verwendung von im Vergleich härterer Strahlung, ohne Einbußen bei der diagnostischen Bildqualität, eingesetzt. Erste Ergebnisse deuten auf eine Dosisverringerung von über $20 \%$ im klinischen Einsatz hin. Zusammenfassung: Das Film-Folien-System Min-R EV zeigt gegenüber den Vorläufermodellen deutlich verbesserte technische Eigenschaften. Es konnte in der klinischen Routine erfolgreich eingesetzt werden. Die Dosisverringerung bei Verwendung des neuen Systems ist aus strahlenhygienischer Sicht sehr begrüßenswert.

\section{P45}

\section{BIRADS V Klassifizierung eines benignen Granularzelltumors der Mamma - ein Fallbericht}

*Andreas Cramer, Silke Jacob, Hans Bielmeier, Dieter Kranzfelder Missionsärztliche Klinik Würzburg, Würzburg, Deutschland

Es wird über den Fall einer mammographischen BIRADS V Klassifikation eines histologisch benignen Granularzelltumors berichtet.

Die 50 jährige Patientin stellte sich mit einem palpablen Tumor der rechten Mamma vor. Eine daraufhin durchgeführte diagnostische Mammographie ergab einen hoch malignitätsverdächtigen Herdbefund. Eine zusätzlich veranlasste MRT-Untersuchung der Brust bestätigte den Verdacht auf Vorliegen eines Mammakarzinoms. Mammasonographisch zeigte sich ein unscharf begrenzter, inhomogener, hypodenser Herdbefund mit deutlichem dorsalem Schallschatten, so dass auch diese Untersuchung den Verdacht auf einen malignen Prozess zu bestätigen schien.

Präoperativ wurde zur Diagnosebestätigung eine sonographisch gesteuerte Stanzbiopsie des Herdes im unteren äußeren Quadranten der rechten Mamma durchgeführt.

Histologisch zeigten sich überraschenderweise Anteile eines benignen Granularzelltumors. Daraufhin wurde lediglich eine lokale Tumorexzision im Gesunden durchgeführt. Das stanzbioptische Ergebnis konnte durch die histologische Aufarbeitung des 1,8 cm großen Gesamttumors bestätigt werden. Dieser wurde weiterhin als benigne eingestuft, da er sämtliche typische Veränderungen der malignen Variante wie Nekrosen, eine deutliche nukleäre Pleomorphie, erhöhten Zellgehalt, zahlreiche Mitosen oder einen deutlich erhöhten Proliferationsindex vermissen ließ.

Es handelt sich somit um den seltenen Fall eines mammographisch, magnetresonanztomographisch und sonographisch sicher als maligne eingestuften Tumors, der in der histologischen Befundung als benigner Granularzelltumor klassifiziert wurde. 
Zytologische Untersuchung der Aspirationsflüssigkeit bei der stereotaktischen 11G-Vakuumbiopsie der MammaUnnötiger Aufwand oder wichtige Zusatzuntersuchung?

Th. Diebold ${ }^{1}$, J. Peters ${ }^{1}$, Ch. Solbach ${ }^{2}$, V. Jacobi ${ }^{1}$, S. Petrich ${ }^{2}$, G. v. Minckwitz ${ }^{2}$, Ch. Herzog ${ }^{1}$, A. Bug ${ }^{3}$, M. Kaufmann ${ }^{2}$, Th. Vog ${ }^{1}$

${ }^{1}$ Universitätsklinik Frankfurt, Institut für Diagnostische und Interventionelle Radiologie, Frankfurt, Deutschland

${ }^{2}$ Universitätsklinik Frankfurt, Klinik für Gynäkologie und Geburtshilfe, Frankfurt, Deutschland

${ }^{3}$ Universitätsklinik Frankfurt, Senckenbergisches Institut für Pathologie, Frankfurt, Deutschland

Zielsetzung: Evaluierung der Menge und der Zusammensetzung der Aspirationsflüssigkeit der stereotaktischen 11G-Vakuumbiopsie in Abhängigkeit von dem histologischen Ergebnis. Material und Methodik: Bei 286 Pat. wurde die Aspirationsflüssigkeit hinsichtlich der Menge sowie der Zusammensetzung ausgewertet sowie die Abhängigkeit der Blutungsmenge von der Dignität der biopsierten Läsion untersucht. Ergebnisse: Bei keiner der Biopsien mit einer benignen Histologie der gewonnenen Präparate wurden in der zytologischen Untersuchung der Aspirationsflüssigkeit maligne Zellen nachgewiesen. Jedoch wurde bei $21,9 \%$ der histologisch malignen Läsionen auch in der Aspirationsflüssigkeit maligne Zellen und bei 6 Patientin Verkalkungen im Zentrifugat der Aspirationsflüssigkeit nachgewiesen. Bei den benignen Zellen wurden am häufigsten Fettz. (40\%), Mammaep. (32\%), Stromaz. (12,5\%) und Hautep. (3\%) nachgewiesen. Bei 12\% wurden keine Epithelien gefunden.. Der durchschnittliche Blutverlust betrug 22,5 ml. Bei 39 Biopsien betrug die Blutungsmenge mehr als $60 \mathrm{ml}$, wobei das Verteilung maligne/benigne 19/20 betrug, also keine erhöhten Blutungsmengen bei den malignen Tumoren beobachtet. Die maxinale Blutungsmenge von $230 \mathrm{ml}$ wurde bei einer massiv ausgeprägten Mastopathie nach nur 1 Rotation nachgewiesen. Schlussfolgerungen: In Anbetracht der Anzahl der durchgeführten zytologischen Untersuchungen ist die Wahrscheinlichkeit eines falsch negatives Biopsieergebnis aufgrund einer «Aspiration» des malignen Gewebes nahezu auszuschliessen. Bei besonders schwierigen Biopsien sollte aufgrund der besonders bei kräftiger Haemorrhagie häufig nachgewiesenen «Aspiration» von epithelialen Strukturen in den Auffangbehälter eine zytologische Untersuchung der Aspirationsflüssigkeit erwogen werden. Die Blutungsmenge bei der Vakuumbiopsie stand im eigenen Kollektiv in keinem Zusammenhang mit der Dignität der Läsion.

\section{P47}

\section{Evaluierung des klinischen Einsatzes der 8G-Vakuumbiopsie} (VB) bei der Abklärung von BI-RADS IV Läsionen

Th. Diebold ${ }^{1}$, J. Peters ${ }^{1}$, Ch. Solbach ${ }^{2}$, V. Jacobi ${ }^{1}$, S. Petrich ${ }^{2}$, G. v. Minckwitz ${ }^{2}$, Ch. Herzog ${ }^{1}$, A. Bug ${ }^{3}$, M. Kaufmann ${ }^{2}$, Th. Vogl ${ }^{1}$ ${ }^{1}$ Universitätsklinik Frankfurt Institut für Diagnostische und Interventionelle Radiologie, Frankfurt, Deutschland ${ }^{2}$ Universitätsklinik Frankfurt, Klinik für Gynäkologie und Geburtshilfe, Frankfurt, Deutschland

3Universitätsklinik Frankfurt, Senckenbergisches Institut für Pathologie, Frankfurt, Deutschland

Zielsetzung: Evaluierung des klinischen Einsatzes der 8G-Vakuumbiopsie (VB) bei der Abklärung von BI-RADS IV Läsionen. Material und Methoden: Bei 38 Patientinnen mit 39 BI-RADS IV Läsionen wurden stereotaktische 8G-VB (Mammotome, Ethicon Endosurgery/Fischertisch) durchgeführt. Die Auswertung beinhaltete das histologische Ergebnis der minimal invasiven Biopsie und der Operation, die benötigte Zeit, die Anzahl der Rotationen/gewonnenen Biopsate, die Größe der Läsionen, das Ausmaß der Resektion sowie die Komplikationen. Ergebnisse: Die Biopsien waren bei 37/39 Läsionen erfolgreich. 36 wurden komplett und $1>50 \%$ entfernt. Bei 2 Biopsien konnten keine repräsentativen Biopsate gewonnen weden, da die Läsion nach Insertion und Reinsertion der Nadel zu weit entfernt waren. Alle bis auf 2 Biopsien (Hämatombildung) wurden ohne klinisch relevante Komplikationen und nach Gewinnung von ausreichend Gewebe beendet (O 16 Biopsate, 1,58 Rotationen). Die durchschnittlich benötigte Zeit betrug 26,5 Minuten, die Größe betrug $2,3 \mathrm{~cm}$. Die Histologien der Vakuumbiopsien waren: ADH (1), DCIS (13) und ID-Ca (1). Bei 12/13 initialen DCIS- Histologien fand sich bei der Op entweder kein DCIS mehr (3) oder bestätigte sich das DCIS (9). Bei einer initialen DCIS-Histo wurde im Op-Präparat ein ID-CA nachgewiesen (Underestimation rate 7,7\%). Die bisherigen Nachkontrollen zeigten keinen Hinweis für falsch negative Biopsien. Schlussfolgerungen: Im Vergleich zu den $11 \mathrm{G}-\mathrm{VB}$ waren die Blutungskomplikationen gering höher und die «underestimation rate» gering niedriger. Die für die selbe Größe an Microkalzifikationen benötigte Zeit ist mit der 8G-VB geringer und es können größere Areale komplett reseziert werden. Die Methodik des neuen ST-Treibers ist leicht zu erlernen und standardisiert das Vorgehen.

\section{P48}

\section{Leitliniengerechte Drahtmarkierung klinisch okkulter Mammatumore}

*Volker F. Duda, Karin Bock, Eva Viktoria Sax, Matthias Kalder Uwe Wagner, Klaus-Jochen Klose

Senologische Diagnostik, Philipps-Universität Marburg, Marburg, Deutschland

Zielsetzung: Die S3-Leitlinie Brustkrebsfrüherkennung fordert die präoperative Drahtmarkierung jedes nicht tastbaren Mammabefundes. Der Draht soll den jeweiligen Befund wenn möglich penetrieren und um nicht mehr als $2 \mathrm{~cm}$ überragen. Sollte dieses Ziel nicht erreicht werden können, ist eine Platzierung des Drahtes in einer Entfernung zum Herdrand von weniger als $1 \mathrm{~cm}$ anzustreben. Materialien und Methoden: Im Arbeitsbereich Senologische Diagnostik des Klinikums der Philipps-Universität Marburg wurden im Jahr 2003242 klinisch okkulte Mammabefunde drahtmarkiert. Präoperativ lag in allen Fällen eine Mammographie und Mammasonographie vor. Alle Befunde wurden apparativ-gestützt mit einem sogenannten X-Draht (Fa. Cook) markiert und nach der definitiven Platzierung des Drahtes geröntgt. Intraoperativ wurden alle Präparate in 2 Ebenen radiographiert und bei Bedarf auch sonographiert. Ergebnisse: Von den 242 Befunden zeigten 133 (55\%) ein sonographisches, 109 $(45 \%)$ ein mammographisches Ziel (Herdbefund in 20, Mikrokalk in 89 Fällen). Sonographisch konnte in 105 Fällen das primäre Markierungsziel erreicht werden. Mammographisch fand sich das $\times$ dagegen überwiegend (in 65 Fällen) in weniger als $1 \mathrm{~cm}$ Entfernung vom oder im Befund, $11 \mathrm{mal}$ allerdings auch weiter als $1 \mathrm{~cm}$ vom Befund entfernt. In 24 Fällen wurden Mikrokalkstraßen von jeweils 2, sogenannten Konzeptnadeln in die Zange genommen. Insgesamt konnte nur bei einem mammographisch gesehenen Herd und bei 3 Mikrokalkarealen die Entfernung beim Ersteingriff nicht erreicht werden. Zusammenfassung: Sonographisch und mammographisch gestützte Markierungstechniken klinisch okkulter Mammatumoren sind in der Lage, die Qualitätsanforderungen der S3-Leitlinie Brustkrebsfrüherkennung zu erfüllen.

\section{P49}

\section{Mammasonographie zur Beurteilung des Therapieerfolges während der neoadjuvanten Chemotherapie des} Mammakarzinoms

\section{Anne Egbe, *Oumara Camara}

Klinik für Frauenheilkunde und Geburtshilfe, Abt. Frauenheilkunde, Jena, Deutschland

Die Anzahl der primär mit neoadjuvanter Chemotherapie behandelten Mammakarzinome $>2 \mathrm{~cm}$ Durchmesser nimmt beständig zu. Dabei werden in den in Deutschland durchgeführten Therapiestudien unterschiedliche Methoden zur Evaluation des Therapieerfolges eingesetzt. Hierzu gehören neben der reinen Palapation des fraglichen Herdes die Mammographie, Mammasonographie und wenn möglich das Mamma-MRT. Dabei zählen auf Grund des Kosten-Zeit-Aufwandes die Palpation und zunehmend auch die Mammasonographie zu den gängigen Methoden. Wegen der hohen Fehlerrate bei der subjektiven Tumorgrößeeinschätzung mittels Palpation vor allem bei wechselnden Untersucher ist von einer alleinigen Palpation jedoch abzuraten. Aus unserer Sicht ist die Mammasonographie eine valide Methode zeitnah das Tumoransprechen auf die neoadjuvante Chemotherapie zu beurteilen. Methode: Wir untersuchten 40 Patientinnen in einem Zeitraum von etwa 1,5 Jahren mit Tumorstadium T2-3. Alle Frauen erhielten eine neoadjuvante Therapie und es wurde zu 
jedem Zytostatikazyklus die Mammasonographie durchgeführt. AbschlieBend wurde die sonographische Remissionsrate festgelegt, unterteilt in stabile Erkrankung, Progression, Partialremission und Komplettremission und mit dem histologischem Ergebnis der anschließenden Operation verglichen. Zusätzlich erhielten alle Patientinnen eine Mammographie vor während und nach Ende der Chemotherapie, zum Teil auch MammaMRT. Ergebnisse: Die sonographisch belegte Progression, stabile Erkran kung und Partialremission konnte in allen Fällen auch histologisch belegt werden. Bei der Komplettremission zeigten sich in $50 \%$ der Fälle auch eine histologische Komplettremission, in den übrigen Fällen zeigten sich mikroskopisch Tumorzellnester im reaktiv verändertem Gewebe oder ypT1a. Zusammenfassung: Die Untersuchung konnte die Praktikabilität und vor allem Validität der Mammasonographie deutlich belegen. Daher erscheint die sonographische Verlaufskontrolle bei neoadjuvanter Chemotherapie empfehlenswert.

P50

\section{Juvenile Papillomatose (JP) der weiblichen Brust} (Swiss Cheese Disease)

*Hilke Frese', Ralf Ohlinger ${ }^{1}$, Günther Schwesinger ${ }^{2}$ Günther Köhler

${ }^{1}$ Klinik und Poliklinik für Frauenheilkunde und Geburtshilfe der

Ernst-Moritz-Arndt-Universität, Greifswald, Deutschland;

${ }^{2}$ Institut für Pathologie der Ernst-Moritz-Arndt-Universität, Greifswald, Deutschland

Zielsetzung: Die juvenile Papillomatose ist eine sehr seltene benigne Brustdrüsenerkrankung. Anhand eines Fallbeispieles soll das Krankheitsbild, die Diagnostik und Differentialdiagnostik sowie das therapeutisch Procedere unter besonderer Berücksichtigung der Mammasonografie erörtert werden. Material und Methoden: Beschrieben wird ein Fall einer 16-jährigen Patientin. Palpatorisch fand sich ein zirka $8 \mathrm{~cm}$ großer verschieblicher Knoten in beiden oberen Quadranten der Brust, der erstmals 6 Monate zuvor getastet wurde. Sonografisch zeigte sich eine ovale, gut abgrenzbare, echoarme heterogene Läsion mit multiplen zystischen Foci. Die FNAZ ergab Epithelzellen und eosinophiles amorphes Material ohne Anhalt für Malignität. Die Diagnosesicherung erfolgte durch die Hochgeschwindigkeitsstanzbiopsie bevor der Befund großzügig im Gesunden entfernt wurde. Ergebnisse und Diskussion: Die Atiologie der JP ist ungeklärt. Es wird eine hormonelle Dysbalance auf der Hypophysen-OvarAchse diskutiert, konnte aber nicht bei allen betroffenen Patientinnen nachgewiesen werden. Auffällig ist die Belastung der Patientinnen durch ein Mammakarzinom in der Familienanamnese, ferner scheinen die $\mathrm{Pa}$ tientinnen selbst ein erhöhtes Risiko zu haben, später an einem Mammakarzinom zu erkranken. Klinisch und mammografisch ist die Vorhersage einer JP nicht möglich. Die Sonografie hingegen ist hilfreich und ermöglicht eine relativ gute Abgrenzung gegenüber einer einfachen Zyste, einem Fibroadenom, einem phylloiden Tumor oder einem intrazystischem Papillom bzw. Karzinom. Die Diagnosesicherung sollte präoperativ über eine Stanzbiopsie erfolgen. Eine offene Biopsie mit Exzision im Gesunden wird aufgrund einer möglichen späteren Karzinomenstehung angeraten.

P51

\section{Progredienter monströser Mammatumor: Angiosarkom}

*Nadir Ghanem ${ }^{1}$, Almut Einert ${ }^{1}$, Bettina Randelzhofer ${ }^{2}$,

M. Orlowska-Volk ${ }^{3}$, Mathias Langer

${ }^{1}$ Abt. Röntgendiagnostik, Radiologische Universitätsklinik Freiburg Freiburg, Deutschland

${ }^{2}$ Abt. Frauenheilkunde, Universitäts-Frauenklinik Freiburg, Freiburg, Deutschland

${ }^{3}$ Sektion Zytopathologie des Pathologischen Instituts der Universitäts-Frauenklinik, Freiburg, Deutschland

Zielsetzung: Fallvorstellung eines seltenen Brusttumors, dem Angiosarkom der Brust. Materialien und Methodik: Darstellung der bildgebende Diagnostik (US, Mammographie, und MRT) der Brust, nuklearmedizinische Untersuchungsverfahren (Skelettszintigraphie und PET) sowie Schnittbilddiagnostik von der CT und der MRT bei einer 27 jährige Pa- tientin mit expansiv raumfordernd wachsendem Brusttumor. Eine Stanzbiopsie wurde durchgeführt. Ergebnisse: Bildgebende Verfahren der Brust wiesen einen soliden expansiv wachsenden Tumor bei einer deutlich vergrößerten rechten Brust auf. Nuklearmedizinische und schnittbildgebende Verfahren detektierten eine Fernmetastasierung. Eine Sanierung des Primärtumors im Bereich der rechten Brust mit dem Versuch einer R0-Resektion wurde empfohlen. Dieses wurde von der Patientin abgelehnt. Zusammenfassung: Das primäre Angiosarkom.der Brust ist ein sehr seltener hochmaligner Tumor mit nachfolgender Fernmetastasierung. Bildgebende Verfahren der Brust und des gesamten Körpers sind von entscheidender Bedeutung für die adäquate Therapie dieser Patientinnen.

P52

Radiäre Narbe/ komplex sklerosierende Läsion der weiblichen Brustdrüse

\section{Wertigkeit der Sonographie in der non-invasiven Diagnostik}

*Susanne Grunwald ${ }^{1}$, Ralf Ohlinger ${ }^{1}$, Hilke Frese ${ }^{1}$, Günther Schwesinger ${ }^{2}$, Anja Kühl' ${ }^{1}$, Anja Schimming ${ }^{3}$, Günter Köhler ${ }^{1}$ ${ }^{1}$ Klinik und Poliklinik für Frauenheilkunde und Geburtshilfe,

Greifswald, Deutschland

${ }^{2}$ Institut für Pathologie, Greifswald, Deutschland

3nstitut für diagnostische Radiologie der

Ernst-Moritz-Arndt-Universität, Greifswald, Deutschland

Problemstellung: Die radiäre Narbe/komplex sklerosierende Adenose als benigne Läsion hat histopathologisch Ähnlichkeit zum tubulären Karzinom, klinisch ist sie nur schwer und mammographisch nicht von einem Malignom zu unterscheiden. Mit dieser Arbeit soll die Wertigkeit der Sonographie in der präoperativen Diagnostik zur möglichen Verminderung der falsch positiven Dignitätsvorhersagen und damit Reduktion der offenen Biopsien aufgezeigt werden. Methode: Insgesamt wurden 6 Fälle einer radiären Narbe in einer retrospektiven Untersuchung klinisch, mammographisch, sonographisch und pathologisch-anatomisch ausgewertet. Ergebnisse: Alle Patientinnen waren über 50 Jahre alt (Mittelwert 66 Jahre). Bei der klinischen Untersuchung ließ sich bei 2 von 6 Patientinnen ein palpabler Befund erheben. Mammographisch war bei einer Frau (ACR 4) keine Aussage möglich, bei den anderen 5 Frauen wurde eine typische Verdichtung mit strahlenförmigen Ausläufern/Spikulae beschrieben. Diese Herde sind alle als malignomsuspekt (BI-RADS IV) bzw. maligne (BI-RADS V) eingestuft worden. Im Brustultraschall konnten alle Herdbefunde dargestellt werden. Übereinstimmend zeigten sich echoarme, inhomogene Läsionen, in den meisten Fällen mit irregulärem Rand sowie mit dorsalem Schallschatten und Unterbrechung der Umgebungsarchitektur. Die sonographische Dignitätseinschätzung deckte sich bei allen Patientinnen mit der mammographischen BI-RADS Klassifikation (Sono-analog-BI-RADS IV und V). Schlussfolgerungen: Einzelne eindeutige Dignitätskriterien in der Sonographie ließen sich nicht herausarbeiten, jedoch ist in der Gesamtschau der Befunde ein fast «typisches sonographisches Erscheinungsbild» der radiären Narbe zu finden. Die erhoffte Reduktion unnötiger offener Biopsien scheint unter B-mode Bedingungen z. Zt. nicht möglich. Inwieweit andere Ultraschallverfahren, wie z. B. die Sono-Elastographie (Elastizitätsdarstellung in Echtzeit) dazu beitragen können, müssen zukünftige Studien zeigen.

\section{P53}

\section{Erste klinische Erfahrungen mit einem neuartigen digitalen Mammographiesystem}

*Grit Häntschel, Bettina Dalitz, Katharina Eberle, Dieter Gosch, Thomas Kahn

Klinik und Poliklinik für Diagnostische Radiologie, Universität Leipzig, Leipzig, Deutschland

Zielsetzung: Im Juli 2003 wurde in unserer Klinik eine digitale Mammographieeinheit in Betrieb genommen. Wir berichten über erste klinische Erfahrungen. Material und Methode: Das System Selenia der Fa. LORAD verfügt über einen $24 \times 29 \mathrm{~cm}$ großen Selendetektor zur direkten Umwandlung von Röntgenstrahlen in ein digitales Signal. Der großflächige Detektor ermöglicht es, dass auch sehr große Mammae vollstän- 
dig erfasst werden können. Bei einer Pixelgröße von $70 \mu \mathrm{m}$ wird eine Auflösung von $>7 \mathrm{Lp} / \mathrm{mm}$ erreicht. Die Befundung erfolgt an einer «SELENIA»-Soft-Copy-Workstation mit speziell für die Mammographie zugelassenes hochauflösenden Röhrenmonitoren. Nach subjektiver Einschätzung wurde die räumliche Auflösung der Mammographie-Aufnahmen als ausreichend, die Kontrastauflösung als sehr gut bewertet. Die Vorteile dieses digitalen Systems liegen nach den klinischen Erfahrungen in der besseren Kontrastauflösung bei dichtem Drüsengewebe und hier besserer Erkennbarkeit von Mikrokalk. Um dies zu objektivieren, wurde eine Phantomstudie durchgeführt. Es wurden 2 Phantome (Phantom MPW-01, SIB-Phantom) mit einem konventionellen System (Mammomat 3000, Fa Siemens)sowie mit dem oben beschriebenen digitalen System geröntgt. Beide Phantome weisen Gruppierungen von Körnchen (Mikrokalkäquivalent), lineare Fäden (Äquivalent für fibröse Strukturen) sowie rundliche Massen (Herdbefundäquivalent) in getrennter Anordnung auf. Ergebnisse: Es zeigt sich eine bessere Detailerkennbarkeit für Mikrokalk und fibröse Strukturen unter Verwendung des genannten digitalen Mammographiesystemes, die Überlegenheit in Bezug auf die Detektion von Herdbefunden ist dagegen weniger deutlich. Schlussfolgerung: Insbesondere aufgrund der Möglichkeiten der digitalen Bildnachverarbeitung und der Monitorbefundung ist das System aufgrund des besseren Kontastverhaltens in der Detailerkennbarkeit trotz geringerer Ortsauflösung den konventionellen Film-Folien-Aufnahmen überlegen

\section{P54}

\section{Vergleich der Expression der Matrilin-Proteine in normalem Brust-Gewebe mit Mammakarzinom-Gewebe aus verschiedenen klinischen Stadien der Erkrankung}

\section{*Nik Hauser ${ }^{1}$, Jasmin Windisch' ${ }^{1}$, Raimund Wagener ${ }^{2}$,} Mats Paulsson ${ }^{2}$, Rolf Kreienberg ${ }^{1}$, Helmut Deissler ${ }^{1}$

'Universitäts-Frauenklinik, UIm, Deutschland

${ }^{2}$ Institut für Biochemie, Medizinische Fakultät der Universität zu Köln, Deutschland

Wachstum, Invasion und Metastasierung maligner Tumoren hängen neben den veränderten zellulären Wechselwirkungen auch von Interaktionen mit Protein-Komponenten der extrazellulären Matrix (ECM) ab Wir lernen zunehmend, dass auch der Umbau der ECM, an dem Stromazellen und Fibroblasten beteiligt sind, ein entscheidender Prozess in der Tumorprogression spielt

Wir konnten bereits beschriebene Proteinkomponenten der ECM, die Familie der Matriline-1 bis -4, in Brustkrebs-Gewebeproben durch Verwendung Isoform-spezifischer Antikörper nachweisen. Zusätzlich untersuchen wir Fragmente dieser Matrixproteine in entsprechenden Blutproben mit dem Ziel eine prognostische und prädiktive Signifikanz nachzuweisen. Wir konnten zeigen, dass Matrilin-1 und -2 homogen im Mammakarzinomgewebe vorkommen; Matrilin-4 konnte nur zwischen KarzinomzellAnsammlungen nachgewiesen werden, jedoch nicht im Stroma. Matrilin-3 wird, im Vergleich zu Matrilin-4, in den Gewebeproben der Mammakarzinompatientinnen schwächer exprimiert. Im Vergleich zum Karzinom kann Matrilin-1 und -3 im gesunden Brustgewebe nicht nachgewiesen werden und Matrilin-2 und -4 sind diffus in der Matrix verteilt. Interessanterweise ist Matrilin-3 schwächer im Tumorgewebe von Patientinnnen mit einer metastasierten Erkrankung exprimiert als in Karzinomgewebe von Patientinnen mit einer loco-regionären Erkrankung. Die Untersuchung sequenzieller Extrakte von Tumorgewebe durch Western Blots zeigte, dass diese nicht-kollagenen Matrixproteine nur partiell solubilisiert werden können und zur vollständigen Extraktion eine Entfernung zweiwertiger Kationen notwendig ist. Dies deutet auf eine Verankerung dieser Proteine in der Matrix über divalente Kationen wie Kalzium un Magnesium hin. Die nachgewiesenen Degradationsprodukte und/oder Splice-Varianten der Proteine im Tumorgewebe werden jetzt weiter untersucht. Diese vorläufigen Resultate liefern einen weiteren Hinweis, dass eine Neuorganisation der ECM während der Entstehung und Progression eines Mammakarzinoms eine wichtige Rolle zu spielen scheint.

\section{P55}

Mammografie, Mammasonografie und klinische Tastuntersuchung - Einfluss auf die Wahl der systemischen Therapieentscheidung beim Mammakarzinom

${ }^{*}$ Katharina Heusinger ${ }^{1}$, Kathrin Imhoff', Christian Löhberg ${ }^{1}$, Achim Magener ${ }^{2}$, Thomas Papadopoulos ${ }^{2}$.

Rüdiger Schulz-Wendtland ${ }^{3}$, Matthias W. Beckmann ${ }^{1}$ Peter A. Fasching

${ }^{1}$ Unifrauenklinik Erlangen, Erlangen, Deutschland

${ }^{2}$ Institut für Pathologie, Universität Erlangen, Erlangen, Deutschland ${ }^{3}$ Institut für diagnostische Radiologie, Universität Erlangen, Erlangen, Deutschland

Einleitung: Die Therapieentscheidung für oder gegen eine primär systemische Chemotherapie wird anhand der klinischen Tumorgröße gefällt Zur Beurteilung der Tumorgröße stehen verschiedene Verfahren zur Verfügung: 1) Mammografie (MG), 2) Brustultraschall (MS) und 3) die klinische Tastuntersuchung. Ziel dieser Analyse war die Bestimmung der prädiktiven Wertigkeit der angegebenen Verfahren zur Vorhersage der tatsächlichen Tumorgröße. Methode: Von 01/01 bis 12/03 wurden in der Unifrauenklinik 965 Patientinnen mit Mammakarzinom primär operiert. Anhand einer Stichprobe von 512 Patientinnen wurde die Tumorgröße anhand der Mammografie (mgT) und die zweidimensionale ultrasonografische Tumorgröße (msT) standardisiert vermessen. Ebenso wurden die klinischen Tastgröße (cT) vor der Operation erfasst. Die angegebenen Befunde wurden mit der pathologischen Tumorgröße (pT) korreliert. Die prädiktive Wertigkeit zur Vorhersage einer Tumorgröße $>2 \mathrm{~cm}$ mittels ROC-Kurven bestimmt. Ergebnisse: Die beste Korrelation mit pT zeigte $\operatorname{mgT}$ mit $r(\mathrm{pT} / \mathrm{mgT})=0,705 . \mathrm{r}(\mathrm{pT} / \mathrm{msT})=0,665$ lag unter $\mathrm{r}(\mathrm{pT} / \mathrm{cT})=0,694$. Zur Vorhersage eine Tumorgröße $>2 \mathrm{~cm}$ war mgT mit einer Fläche unter der ROC-Kurve von AUC =0,861 besser geeignet als msT mit AUC = 0,839 und cT mit AUC $=0,821$. Alle Parameter behielten ihre Signifikanz in der multivariaten Analyse, so dass bei der Kombination der Verfahren eine AUC von 0,874 erreicht werden konnte. Zusammenfassung: MG, MS und die klinische Tastuntersuchung eignen sich unabhängig voneinander zur Vorhersage der pathologischen Tumorgröße. Am besten eignet sich die MG als Einzelverfahren zur Bestimmung der realen Größe. Bei der Kombination der Verfahren konnte eine weitere Verbesserung der Vorhersagekraft erreicht werden, so dass die komplementäre Mammadiagnostik der Standard bei der Größenbestimmung des Tumors sein sollte.

\section{P56}

\section{Leiomyom der Brust - Klinische, mammographische und} sonographische Zeichen in Beziehung zum feingeweblichen Befund

${ }^{*}$ Hans Heyer ${ }^{1}$, Ralf Ohlinger ${ }^{1}$, Christian Woenckhaus ${ }^{2}$, Günter Köhler ${ }^{1}$ Universitätsfrauenklinik Greifswald (Direktor Prof. Dr. W. Straube), Greifswald, Deutschland

${ }^{2}$ Institut für Pathologie der EMAU Greifswald

(Direktor Prof. Dr. G. Lorenz), Greifswald, Deutschland

Fragestellung: Leiomyome des Brustdrüsenkörpers finden sich sehr selten. Es werden areoläre und parenchymatöse Leiomyome unterschieden. Klinisch imponiert das Leiomyom als Knoten, mammographisch als Rundherd. Sonographische Kriterien sind indifferent und in der Literatur bisher nur vereinzelt beschrieben wurden. Die Darstellung eines eigenen Falles ermöglicht weitere sonographische Kriterien aufzuzeigen. Methodik: Klinische, mammographische und mammasonographische Kriterien eines intramammären Leiomyoms der Brust wurden erfasst. Die Diagnose konnte operativ histologisch gesichert werden. Ergebnisse: Klinisch fiel das tiefe Leiomyom der Brust durch Hauteinziehung und fehlende Verschieblichkeit und somit Malignomverdacht auf. Die mammographisch sichtbare verdichtete und scharf bis unscharf begrenzte Struktur (BIRADS IV) ist identisch zu den gefundenen Literaturangaben. Mammasonographisch wurde das Leiomyom als hypodense, glatt begrenzte, inhomogene Struktur mit retrotumorösen Schallschatten dargestellt (EUSOMA IV). Die Cooper-Ligamente waren unterbrochen und dopplersonographisch zeigte sich ein zentrales Tumorgefäß. Die Schallabschwächung, die hypodensen Eigenschaften, der echoreiche Randsaum und das zentrale Tumorgefäß sind weitere sonographische Eigenschaften die ein paren- 
chymatöses Leiomyom beschreiben können. In der Literatur ist diese Tumorentität bisher als isodense bis hyperdense, homogene Läsionen ohne Schallschattenphänomene oder nur mit dorsalen Schallverstärkungen be schrieben wurden. Schlussfolgerung: Ein parenchymatöses Leiomyom der Brust kann durch eine retrotumoröse Schallabschwächung, eine hypoden se Echogenität, einen echoreichen Randsaum und ein zentrales Tumorgefäß in der Mammasonographie in Erscheinung treten. Eine eindeutige präoperative Dignitätsvorhersage bezüglich, Malignität oder Benignität erscheint zur Zeit weder durch Palpation noch durch Bildgebung möglich.

\section{P57}

\section{Mikro-Duktoskopie mit einem 0,55 mm Endoskop zur Sichtbeurteilung von Milchgangsveränderungen}

\section{*Volker R. Jacobs ${ }^{1}$, Marion Kiechle ${ }^{1}$, Hansgeorg Schaaf ${ }^{2}$,} Stefan Paepke ${ }^{1}$

${ }^{1}$ Frauenklinik, Technische Universität, München, Deutschland;

${ }^{2}$ PolyDiagnost, Pfaffenhofen, Deutschland

Zielsetzung: Standarddiagnostik wie Ultraschall, Mammo- oder Galaktographie bei unklaren Milchgangsveränderungen können nur indirekte Information über die Ursache von blutiger Sekretion geben. Ambulante Mikro-Endoskopie mit einem Milchgangsendoskop mit nur 0,55mm kann zusätzlich ein direktes Bild der Läsion ermöglichen und helfen, den Entscheidungsprozess zu vereinfachen und eine explorative Mamma-PE zu vermeiden. Wir berichten über Erfahrungen mit dieser neuen Technik. Materialien und Methoden: Wir benutzen ein PolyDiagnost LaDuScope ${ }^{\circledR}$ System mit einem Ausendurchmesser von 0,55-0,95 mm und einer Arbeitslänge von $75 \mathrm{~mm}$. Die Optik hat einen Aussendurchmesser von nur 0,36-0,76 mm, eine Gesamtlänge von $1200 \mathrm{~mm}, 0^{\circ}$ Direktsicht, ein Blick feld von $70^{\circ}$ und 3,000-6,000 Pixel Auflösung. Die Spülung der Milchgänge wird mittels ansetzbarer Spritze durchgeführt. Der Eingriff erfolgt als ambulante Diagnostik. Die Patientin ist wach; eine geringe Sedierung erleichtert die Anspannung und diskreten Beschwerden insbesondere be retromammillärer Dilatation der Milchgänge. Ergebnisse: Nach Einführung des Duktoskops können die Wände der Milchgänge ohne Beschwerden für die Patientin unter Sicht beurteilt werden. Anstatt der aktiven Bewegung des Endoskops wird dabei das Brustgewebe in Richtung zum Duktoskop bewegt. Die Ergebnisse können zeitgleich der Patientin auf dem Monitor dargestellt und erklärt werden; die gesamte Untersuchung kann zur Befunddokumentation und Auswertung auf CD-Rom aufgenommen werden. Methodenbedingt ist das Monitorbild mit $8-12 \mathrm{~cm}$ Durchmesser etwas kleiner als von der Endoskopie gewohnt. Alle bisherigen 10 Untersuchungen verliefen komplikationslos. Zusammenfassung Die Mikro-Duktoskopie ist eine unkomplizierte zusätzliche Untersuchungsmethode zum Ausschluss von offensichtlicher Malignität bei unklaren Brustgangsveränderungen. Dieses Duktoskop demonstriert Fortschreiten und Nutzung neuester Technologien und einen Trend zur weniger invasiven Sichtbeurteilung von Milchgängen durch Mikro-Endoskopie.

\section{P58}

\section{Mammasonographie als Erstuntersuchung im Vergleich zur Mammographie bei asymptomatischen Frauen der Altersgruppe 40-49 Jahre}

*Ulrike Klug ${ }^{1}$, Ralf Ohlinger ${ }^{1}$, Annette Schimming ${ }^{2}$, Günter Köhler ${ }^{1}$ ${ }^{1}$ Klinik für Frauenheilkunde und Geburtshilfe E. M. A. Universität Greifswald, Deutschland

2Institut für Radiologie, E. M. A. Universität, Greifswald, Deutschland

Zielsetzung: Evaluierung diagnostischer Wertigkeit der Mammasonographie als Erstuntersuchung im Vergleich zur Mammographie bei asymptomatischen Frauen der Altersgruppe 40-49 Jahre. Methoden: Im Zeitraum vom 30.11.1994 bis 23.01.2003 erhielten 1000 Frauen eine BrustultraschallUntersuchung. In der Altersgruppe 40-49 Jahre befanden sich 289 Frauen $(28,9 \%)$, von denen in 146 Fällen (50,5\%) ein Mammographiebefund vorliegt. Nach Erhebung der Anamnese fand die sonographische und anschließend unter Unkenntnis dieses Befundes die mammographische Untersuchung der Brüste statt. Bei auffälligem sonographischen oder mammographischen Befund (BI-RADS 4/5) sollte eine histologische Ab- klärung resultieren. Ergebnisse: In zehn Fällen ergab sich ein auffälliger Befund (BI-RADS 4/5). Zweimal konnte die Diagnose durch die Mammographie, achtmal durch die Sonographie gestellt werden. Eine histologische Abklärung erfolgte in fünf Fällen. In drei dieser Fälle liegen Sonographie- und Mammographiebefund vor. Für die Mammographie ergibt sich eine m/b-Rate von 1:1 und für die Sonographie von 0:3. Fünf sonographisch suspekte Fälle wurden histologisch nicht abgeklärt. Von den 10 auffälligen Befunden befanden sich 9 in teilinvolviertem oder involviertem Gewebe (eine Angabe fehlt), ein Gewebe welches in der Untersuchungsgruppe mit $82,5 \%$ vorherrschte. Eine Korrelation zeigte sich für die Ultraschallbeurteilbarkeit in Abhängigkeit vom Brustdrüsenparenchym (Korrelationskoeffizient R2 = 0,9627). Schlussfolgerung: Die Mammographie weist durch Detektion eines Karzinoms (LCIS) und der guten $\mathrm{m} / \mathrm{b}$-Rate gegenüber der Sonographie Vorteile bei der Beurteilung von teilinvolviertem und involviertem Brustdrüsengewebe auf. Die Ultraschallbeurteilbarkeit steigt mit Zunahme der Dichte des Brustdrüsenparenchyms. Es konnte kein Vorteil der Sonographie gegenüber der Mammographie in der Altersgruppe 40-49 Jahre herausgestellt werden.

\section{P59}

\section{B-Klassifikation: Qualitätsindikator für die Pathologie?}

*Volker Loy , Jörg Linke1, Michael Wolf²

${ }^{1}$ Vivantes Fachbereich Pathologie, Berlin, Deutschland

2Zentrum für Brusterkrankungen, Klinikum Am Urban, Berlin, Deutschland

\section{Zielsetzung:}

1. Lässt sich die B-Klassifikation in der Routinepathologie ohne zusätzlichen Aufwand durchführen?

2. Welche Auswirkungen hat die Klassifikation auf die Pathologie?

3. Wie kann eine Dokumentation ohne zusätzlichen Aufwand erfolgen?

4. Wie sicher ist die Schnellschnittuntersuchung von Mammastanzen unter Routinebedingungen?

Materialien und Methode: Ab 2003 wurden prospektiv alle Mammastanzen entsprechend der B-Klassifikation (NHSBSP) beurteilt. Ab Juli 2003 wurden die Mammastanzen des Zentrums für Brusterkrankungen parallel im Schnellschnitt und am paraffineingebetteten Gewebe untersucht (zwei Fachärzte). Die Dokumentation erfolgte für die Schnellschnittuntersuchung in Form eines 1. Berichtes und für die Paraffinschnittuntersuchung getrennt später als abschließende Begutachtung. Ergebnisse: 797 Stanzen wurden untersucht. B-Klassifikation: B1 5,8\%; B2 40,8\%; B3 1\%; B4 1,5\%; B5a 5,7\%; B5b 44,7\%; B5c 0,4\%. 230 Fälle wurden im Schnellschnittverfahren und nach Paraffineinbettung untersucht. Die B-Kategorie stimmte zwischen Schnellschnitt und Paraffinschnitt überein: 81\%, war nach Paraffineinbettung höher: $15 \%$, kleiner: 4\%. In der relevanten Kategorie B5 wurde die B-Kategorie nach Paraffineinbettung in zwei Fällen zurückgestuft, 5b nach B4, B5b nach B5a. Das verfügbare Gewebe war nach Paraffineinbettung nicht ausreichend beurteilbar, die Untersuchung des Resektates bestätigte jedoch die Schnellschnittbeurteilung. Zusammenfassung: Seit 2003 wurden 797 Mammastanzen histologisch untersucht, darunter 404 Karzinome. 230 Fälle wurden zunächst im Schnellschnitt und danach am Paraffinschnitt begutachtet, das Ergebnis beider Begutachtungen wurde getrennt anhand der B-Klassifikation dokumentiert. Die B-Klassifikation erwies sich dabei als ausgezeichneter Qualitätsindikator, der objektivierbar die diagnostische Qualität in der Pathologie wiedergibt und für die Routinepathologie geeignet ist. Durch den Zwang zur Festlegung wirkt sich die Klassifikation auch auf die Pathologie aus und unterstützt die Qualitätssicherung.

\section{P60}

\section{Mammasonographie - eine Screeningmethode}

*Ralf Ohlinger, Günter Köhler

Klinik für Frauenheilkunde und Geburtshilfe E. M. A. Universität Greifswald, Greifswald, Deutschland

Zielsetzung: Bei asymptomatischen Frauen wurde die Hochfrequenzsonographie als erstes diagnostisches Verfahren eingesetzt, ohne dass zuvor eine Mammographie vorlag. Geprüft werden sollte, ob es gelingt klinisch nicht palpable Karzinome zu entdecken und wie hoch die Anzahl unnötiger offener Biopsien sein wird. Methode: 1000 asymptomatische Frauen 
wurden sonographiert, in 449 Fällen erfolgte im Anschluss eine Mammographie. Ergebnisse: Insgesamt wurden fünf nicht palpable Karzinome entdeckt, je vier davon durch die Sonographie $(3 \times$ pT1b, $1 \times$ pT1c) und die Mammographie. Ein CLIS wurde sonographisch als benigne eingestuft, ein Karzinom mammographisch. Die Rate unnötiger offener Biop sien lag bei $1,7 \%(n=17)$. Histologisch fanden sich darunter z.B. ein Cys tosarcoma phylloides benignum, eine ADH, eine ALH und zwei FA mit Proliferation. Schlussfolgerung: Mit der hochauflösenden Mammasonographie gelingt es bei asymptomatischen Frauen Mammakarzinome zu dedektieren und das in einer Größenordnung wie sie von der Mammographie als Screeningmethode gefordert wird. Ein Anstieg unnötiger offener Biopsien ist nicht zu erwarten. Schlussfolgernd sollte die Sonographie als additive Methode in ein Screeningprotokoll an einer größeren Anzahl von Patienten einbezogen und überprüft werden.

\section{P61}

Sekretorische Brusterkrankungen - Informationsgewinn durch Duktoskopie?

*Ralf Ohlinger, Susanne Grunwald, Günter Köhler

Klinik und Poliklinik für Frauenheilkunde und Geburtshilfe der

Ernst-Moritz-Arndt-Universität Greifswald, Deutschland

Problemstellung: Die Ursachen sekretorischer Erkrankungen der Brust sind, wie z.B. die Mastopathie, meist benigner Natur. Maligne invasive und non-invasive Veränderungen können jedoch auch zu einer Galaktorrhoe führen. Als Präkanzerose sind das Milchgangspapillom, bzw. die Papillomatose mit einer Entartungstendenz von $5-17 \%$ oft eine diagnostische Hürde. Inwieweit die Duktoskopie zur Diagnosefindung bzw. -sicherung und Therapieerleichterung dienen kann, soll im Folgenden erläutert werden. Methode: Die Diagnosekette nach den Leitlinien der Deutschen Röntgengesellschaft umfasst nach Ausschluss einer hormonellen oder entzündlichen Genese die Mammographie, Galaktographie und gegebenenfalls die Sonographie. In der eigenen Einrichtung wird dieses Procedere z. T. abgewandelt und durch andere Verfahren ergänzt, dabei kommen insbesondere die Duktsonographie und die endoskopische Milchgangsdarstellung zum Einsatz. Ergebnisse: Die Vorteile der Duktsonographie sowie der zytologischen oder histologischen präoperativen Diagnosesicherung unter Ultraschallsicht werden vorgestellt. Die sonographische Dignitätsvorhersage und die intraoperative duktoskopische Verdachtsdiagnose korrelieren in über der Hälfte der Untersuchungen mit dem histologischen Befund. Die Aussage der Galaktographie hingegen ist dagegen häufig unzureichend. Schlussfolgerung: Weitere Fallzahlen sind notwendig, um statistisch einen signifikanten Unterschied zwischen den einzelnen Diagnoseverfahren herauszuarbeiten. Des Weiteren ist zu untersuchen, ob die Probeentnahme über einen Arbeitstrokar möglich, und auch in Lokal- bzw. Regionalanästhesie machbar ist. Erst dann ist die Duktoskopie wirklich als diagnostische Maßnahme, gegebenenfalls als Ergänzung /Ersatz zur Galaktographie zu sehen.

\section{P62}

\section{Stand der sonographisch geführten Mamma-Biopsien in Deutschland: erste Ergebnisse der Fragebogenerhebung der AG MIMI}

*Stefan Paepke, W. H. Becker, Volker Duda, Karsten Gnauert, Markus Hahn, Uwe Peisker, D. Scheich, Ute Krainick-Strobel Arbeitsgemeinschaft für Minimalinvasive Mammainterventionen der Deutschen Gesellschaft für Senologie

Einleitung: Die AG Minimal-invasive Mammainterventionen hat sich unter anderem die Erarbeitung von Standards in der minimal-invasiven Diagnostik und die Qualitätskontrolle zum Ziel gesetzt. Als Grundlage erfolgt in einem ersten Schritt die Erfassung der derzeitigen Indikationen, der Methodenwahl und Vorgehensweisen. Aus der Zusammenführung und nach diskussion der bisherigen Erfahrungen aller Arbeitsgruppen soll eine Konsensusempfehlung zur Durchführung der sonographisch gestützten Vacuumbiopsie erarbeitet werden. Prospektive Vergleichsuntersuchungen der Mammotome-Vacuumbiopsie mit der bisher als Standard geltenden Hochgeschwindigkeitsbiopsie stehen zur Zeit noch aus. Material und Methoden: Zur Erfassung des aktuellen Standes der Mam- mabiopsien wurde ein Fragebogen entwickelt (20 Items) und an die AG MIMI Mitglieder per e-mail verschickt (März, zweite Runde Mai 2004). Von den bisher auswertbaren 6 Zentren wurden insgesamt 2885 sonographisch gestützte Hochgeschwindigkeits- und 414 Vacuumbiopsien im Jahr 2003 durchgeführt. Ergebnisse: Im Mittel wurden 11,13 Proben entnommen (1-39). Eine erste Auswertung erfolgt hinsichtlich der Indikationen (Auswertung Zentrum I):

BIRADS II: 5,6\%; BIRADS III: 12,1\%; BIRADS IV: 22,4\%; BIRADS V: $50,0 \%$ und der Zielstellung der sonographisch gestützten Vacuumbiopsie diagnostisch//diagnostisch-therapeutisch//therapeutisch:

Zentrum I: $18,75 \% / 18,75 \% / 62,50 \%$

Zentrum II: $0,00 \% / 100 \% / 0,00 \%$

Zentrum III: $4,5 \% / 0,00 \% / 95,5 \%$

Zentrum IV: $70,0 \% / 20,0 \% / 10,0 \%$

Zentrum V: $36,1 \% / 63,9 \% / 0,00 \%$

Zentrum VI: 79,7\%/20,3\%/0,00\%

Schlussfolgerungen: Die gemeinsame Auswertung zeigt erhebliche Unterschiede zwischen den einzelnen Einrichtungen; insbesondere bei der Zielstellung der Vacuumbiopsie. Dies wiederspiegelt erwartungsgemäss die Situation bei fehlenden Leitlinien. Die Daten dienen als Grundlage für die interdisziplinär zu führende Konsensusdiskussion.

\section{P63}

\section{Spektrum der Tumorentitäten in einer Brustambulanz}

${ }^{*}$ Harald Rott ${ }^{1}$, Anton Scharl ${ }^{2}$, Uwe-J. Goehring ${ }^{3}$, Björn Lampe ${ }^{1}$

${ }^{1}$ Klinikum Leverkusen, Leverkusen, Deutschland

${ }^{2}$ Klinikum St. Marien, Amberg, Deutschland

${ }^{3}$ Johanniter-Krankenhaus, Bonn, Deutschland

Spezialisierung und Zentrumsbildung sowie der vermehrte Fokus der Öffentlichkeit auf Brusterkrankungen führen zu einer vermehrten Zuweisung an spezialisierte «Brustambulanzen». Vorliegende Auswertung liefert einen Überblick über die Tumorentitäten und die minimal-invasive Diagnostik in einer spezialisiserten Ambulanz.

Im Jahre 2003 wurden etwa 1000 Patientinnen in der Brustambulanz des Klinikums Leverkusen untersucht.

Es wurden 19 Punktionszytologien unter US-Kontrolle bei zystischen Herdbefunden (Schmerzen oder belastender Tastbefund) durchgeführt, in keinem Fall war eine Folgeoperation erforderlich.

Es liegen von insgesamt 439 Patientinnen der Frauenklinik histologische Mamma-Befunde vor.

In 267 Fällen wurde die Histologie durch interventionelle Diagnostik Stanzbiopsie, Punchbiopsie, Vakuumbiopsie - in Lokalanästhesie erzielt Bei 116 dieser Patientinnen wurde im Anschluß kein offen-chirurgischer Eingriff durchgeführt.

Der häufigste histologische Befund war mit 177 Fällen das primäre Mammakarzinom (incl. pTi.s.), gefolgt von 75 histologisch gesicherten Fibroadenomen. Es wird die Häufigkeitsverteilung der wichtigsten weiteren benignen (z.B. Phylloidestumor, Papillom, Abszeß) und malignen (z.B. NHL, Sarkom, Metastasen) Läsionen beschrieben und ein Vergleich mit Literaturangaben durchgeführt. Zu seltenen malignen Befunden werden Sonographiebilder demonstriert.

P64

\section{Beeinflussen Rezeptor- und Menopausenstatus die Aussagekraft des Mamma-MRT beim Tumormonitoring nach neoadjuvanter Chemotherapie (PST) bei Patientinnen mit} lokal fortgeschrittenen Mammakarzinom (LABC)?

${ }^{*}$ V. Russu ${ }^{1}$, B. Lex ${ }^{1}$, T. Gilster ${ }^{1}$, K. Pfaffenberger ${ }^{2}$, H. Volkholz ${ }^{3}$, A. H. Tulusan ${ }^{1}$

${ }^{1}$ Frauenklinik, Klinikum Bayreuth $\mathrm{GmbH}$, Bayreuth, Deutschland ${ }^{2}$ MR-Zentrum, Klinikum Bayreuth GmbH, Bayreuth, Deutschland ${ }^{3}$ Institut für Pathologie, Klinikum Bayreuth $\mathrm{GmbH}$, Bayreuth, Deutschland

Zielsetzung: Die PST stellt eine zunehmend angewandte Behandlungsform der präoperativen Tumorreduktion bei Patientinnen mit LABC. Bereits etablierte Methoden zu Beurteilung von Tumorgröße bzw. Tumoransprechen auf PST sind die Palpation, Mammographie und Sonographie, 
jedoch die Sensitivität dieser Methoden ist unzufrieden. Mamma-MRT dagegen scheint als sehr zuverlässige und sensitive Methode zur Brustkrebsentdeckung und Tumormonitoring im Rahmen PST besonders geeignet zu sein. Ziel dieser Studie war zu evaluieren ob Rezeptor- und Menopausenstatus die Aussagekraft des Mamma-MRT beim Tumormonitoring nach neoadjuvanter Chemotherapie (PST) bei Patientinnen mit lokal fortgeschrittenen Mammakarzinom (LABC) beeinflussen. Materialien und Methoden: 120 Frauen im Alter zwischen 29 und 69 Jahren mit LABC (cT2 - cT4) waren Mamma-MRT nach der PST unmittelbar vor OP unterzogen. 37 davon waren sequentiell mit Anthracyklin- and Taxanbasierter Chemotherapie behandelt und 83 erhielten nur anthrazyklinbasierte Chemotherapie. 55\% Patientinnen waren prämenopausal. 78 Frauen hatten rezeptorpositiven Tumor, bei 3 konnte Rezeptorstatus nicht ermittelt werden. Ergebnisse: Menopausen- und Rezeptorstatus scheinen die Aussagekraft des MRT hinsichtlich des zu erwartenden histopathologischen Resttumors nicht zu beeinflussen. Die größte Signifikanz ( $\mathrm{r}=$ 0,995) konnte für rezeptornegative Tumoren postmenopausaler Patientinnen festgestellt werden. Zusammenfassung: Mamma-MRT ist geeignete Methode für die Monitoring des Tumoransprechens unter PST. Menopausen- und Rezeptorstatus scheinen keinen Einfluss auf Sensitivität bzw. Spezifität der Methode zu haben. Tendenziell höhere Signifikanz für rezeptornegative Tumoren postmenopausaler Patientinnen muss noch in den weiteren Studien überprüft werden.

\section{P65}

\section{Ultraschall-gestüzte, invasive Mammadiagnostik an der Universitäts-Frauenklinik (UFK) Basel, CH}

${ }^{*}$ C. Schuller, R. Zanetti, W. Holzgreve, E. Wight

Universitäts-Frauenklinik Basel, Basel, Schweiz

Einführung: An unserer Klinik hat die Abklärung von Läsionen in der Mamma mittels sonographisch geführter transcutaner Biopsiemethoden (US-Biopsie) einen grosse Bedeutung erlangt. Material und Methoden: Von 6/2002 bis 12/2003 wurden an der UFK insgesamt 252 US-Biopsien durchgeführt. Davon 75 Feinnadelpunktionen (FNP), 128 Stanzbiopsien (BARD ${ }^{\circledR}$ System) und 49 Biopsien mittels Mammotome hand held ${ }^{\circledR}$ Die, durch invasive Diagnostik ermittelten feingeweblichen Befunde, wurden entweder mittels offener Biopsie histologisch bestätigt oder, im Falle von, in der Bildgebung und invasiver Diagnostik als benigne eingestuften Läsionen, durch Verlaufskontrollen mit bildgebenden Verfahren weiter verfolgt (6-18 Monate). Ergebnisse: Insgesamt diagnostizierten wir unter den 252 Befunden 25 Karzinome (Ca) und 1 malignes Histiozytom (Cancer rate (CR) 10,4\%). Wir biopsierten 178 Befunde der Kategorie 3 (Kat. 3 analog BIRADS) (ws. benigne), darunter fand sich $1 \mathrm{Ca}$ (CR $0,56 \%$ ). Von 34 Befunden der Kat. 4 (suspekt) waren $4 \mathrm{Ca}, 1$ mal fand sich ein malignes Histiozytom (CR 14,7\%). Unter den 22 Befunden der Kat. 5 (ws. maligne) fanden sich $20 \mathrm{Ca}$ (CR 90,9\%). Die 252 US-Biopsien führten insgesamt $1 \mathrm{mal}$ zu einem falsch negativen Ergebnis (0,39\%). Das ergibt eine Sensitivität von $96 \%$. Die Spezifität liegt bei $100 \%$. Der positive predictive value des Verfahrens liegt bei $100 \%$ und der negative predictive value beträgt $99 \%$. In der Literatur finden sich falsch negative Raten zwischen $0 \%$ und $1,26 \%$, eine Sensitivität zwischen $86 \%$ und $100 \%$, sowie eine Spezifität von 100\%. Schlussfolgerung: Basierend auf unseren Ergebnissen und vergleichbaren Studien ist die US-Biopsie in unseren Händen, ein zuverlässiges Mittel, die Dignität eines Mammabefundes zu bestimmen.
P66

\section{Apokrines Karzinom der Mamma}

${ }^{*}$ Karen Schulz ${ }^{1}$, Ralf Ohlinger ${ }^{1}$, Susanne Grunwald ${ }^{1}$, Günter Schwesinger ${ }^{2}$, Annette Schimming ${ }^{3}$, Günter Köhler ${ }^{1}$

${ }^{1}$ Klinik für Frauenheilkunde und Geburtshilfe,

E. -M. -Arndt-Universität Greifswald, Deutschland

${ }^{2}$ Institut für Pathologie, E. -M. -Arndt-Universität Greifswald, Deutschland

${ }^{3}$ Institut für Diagnostische Radiologie, E. -M. -Arndt-Universität Greifswald, Deutschland

Es wird über eine 50-jährige Patientin mit zunehmenden Beschwerden im Bereich eines seit mehreren Jahren bestehenden Tastbefundes der rechten Brust berichtet. 1979 erfolgte in diesem Bereich eine diagnostische Exstirpation wegen eines benignen Befundes.

Palpatorisch zeigte sich im Bereich der reizlosen Narbe ein derber verschieblicher $2 \mathrm{~cm}$ großer dolenter Tumor. Die Mammographie beschrieb lediglich eine angedeutete gewebliche Raffung rechts (BI-RADS3). Sonographisch stellte sich ein inhomogener, echoarmer, unscharf begrenzter Herdbefund mit unilateralem Schallschatten und Unterbrechung der Cooper-Ligamente sowie eine erhöhte Vaskularisation dar (analog BIRADS4). Es erfolgte die sonographisch gestützte Stanzbiopsie, welche ein invasives relativ großzelliges solides Karzinom mit Granulierung des Zytoplasmas der Tumorzellen ergab.

Anschließend wurden die brusterhaltende Operation und die axilläre Lymphonodektomie durchgeführt.

Der endgültige histologische Befund ergab neben einem DCIS ein invasives apokrines Karzinom: pT2 (2,5 cm), pN1a (2/18), pR0, pL1, pV1, G3, Estrogen-, Progesteronrezeptor positiv. Das Zytoplasma zahlreicher Tumorzellen zeigte sich zart eosinophil mit feiner Granulierung und vereinzelt PAS-positivem Hintergrund.

Die Staginguntersuchungen ergaben keinen Anhalt für Fernmetastasierung.

Es wurde eine adjuvante Chemotherapie, Radiatio und Hormontherapie (Tamoxifen, GnRH-Analoga) empfohlen.

Das apokrine Karzinom ist ein sehr seltener invasiver Tumor und wird in weniger als 1\% aller Mammakarzinome beobachtet. Es sind Tumore, die sich durch großleibige Epithelzellen mit eosinophilem Zytoplasma auszeichnen. Die apokrinen Karzinome sind gut abgrenzbar und treten bevorzugt im 6. Lebensjahrzehnt mit Symptomen wie Knotenbildung, Schmerzen und Mamillensekretion auf. Es werden rasches Tumorwachstum, bevorzugte Lokalisation zentral oder im oberen äußeren Quadranten der rechten Brust und mittlere Größen zwischen 2-5 cm beschrieben. Hinsichtlich der Prognose unterscheidet sich das apokrine Karzinom nicht von invasiv-duktalen Tumoren.

\section{P67}

\section{Epidermiszyste der Brust: klinische, radiologische und patho- logische Korrelation (Case Report)}

*Beate M. Stoeckelhuber, Isabel Rudolf, Stefan Krueger, Hans-Bjoern Gehl, Claudia U. Bergmann-Koester Institut für Radiologie, Universitätsklinikum Schleswig-Holstein, Campus Lübeck, Lübeck, Deutschland

Zielsetzung: Korrelation klinischer, radiologischer und pathologischer Befunde der sehr selten intramammär auftretenden Epidermiszyste Materialien und Methoden: Eine 52-jährige Patientin stellte sich zur Klärung eines palpablen Knotens in der linken Brust vor. Mammographie, Sonographie einschließlich Powerdoppler Sonographie und sonographisch gestützte Hochgeschwindigkeitsstanzbiopsie wurden durchgeführt, das gewonnene Gewebe an das Institut für Pathologie gegeben. Ergebnisse: Mammographisch zeigte sich eine nicht allseits glatt begrenzte Läsion in der linken Brust. Sonographisch war dieser Herd echoarm, gering inhomogen und nicht allseits scharf begrenzt. In der Powerdoppler Sonographie war eine punktförmige Vaskularistaion nachweisbar. Der Befund wurde als BI-RADS 4 eingestuft. Bei der histologischen Aufarbeitung der Stanzzylinder fand sich koriales Bindegewebe und Fettgewebe mit Übergang in eine Zyste, die mit mehrschichtigem Plattenepithel ausgekleidet war; in der Lichtung zwiebelschalenartig angeordnetes Hornmaterial. Zusammenfassung: Die intramammäre Epidermiszyste ist ein sehr seltener 
benigner Brusttumor, der bildgebend als maligner Befund imponieren kann. Aufgrund des bei diesen Läsionen möglichen Größenwachstums sollten sie operativ entfernt werden.

\section{P68}

Darstellung von Mammabefunden mittels der neuen

SonoElastographie bei anschließend histologischer Sicherung

${ }^{*}$ Anke Thomas ${ }^{1}$, Sherko Kümmel ${ }^{1}$, Thomas Fischer ${ }^{2}$, Holger Frey ${ }^{3}$, Jens-Uwe Blohmer ${ }^{1}$

${ }^{1}$ Klinik für Gynäkologie und Geburtshilfe, Charite Mitte, Berlin, Deutschland

${ }^{2}$ Institut für Radiologie, Charite Mitte, Berlin, Deutschland

${ }^{3}$ HITACHI Medical Systems GmbH, Wiesbaden, Deutschland

Zielsetzung: Das neue sonographische Verfahren der Elastographie ist eine Methode zur Darstellung der Gewebeelastizität. Diese Elastizitätsveränderungen können farbkodiert sichtbar gemacht werden. Die Zielsetzung besteht in der Frage ob dies zur Verbesserung einer eindeutigeren und sichereren Einschätzung der Dignität führt. Materialien und Methoden: In einem Zeitraum von 2 Wochen wurden 22 Patientinnen mit einem unklaren Mammabefund (Altersmedian: 56,5 Jahre) sonographisch mit dem HITACHI EUB-8500 (HITACHI Medical Systems GmbH, Wiesbaden) untersucht. Unter Verwendung eines 13-Mhz-Linearschallkopfes wurde neben dem konventionellen B-Bild die Realtime-Elastographie aufgezeichnet. Die Dignitätseinteilung erfolgte durch BIRADS Kriterien (I-V) und einem Elastographie-Score (I-V). Anschließend erfolgte die histologische Sicherung. Dabei wiesen 11 Patientinnen ein Mammakarzinom, 2 ein Carcinoma in situ und 9 einen benignen Herdbefund auf. Ergebnisse: Im B-Bild zeigten 14 Patientinnen einen suspekten Mammabefund, wobei 11 mit einem BIRADS V und 3 mit einem BIRADS IV klassifiziert wurden. Von den anderen 8 Patientinnen, mit sonographisch benignen Befund, ließen sich 6 mit einem BIRADS III und 2 mit BIRADS II klassifizieren. Der Elastographie-Score entspricht in $100 \%$ den BIRADS Kriterien. 12 der 14 Patientinnen mit sonographisch suspektem Herdbefund haben nachweislich ein Mammakarzinom. Die 8 benignen Befunden konnten in 7 Fällen histologisch gesichert werden. Die Spezifität der Methode der Elastographie liegt bei 87,5\% mit einer Sensitivität von $85 \%$. Zusammenfassung: Die Messung der Gewebeelastizität mittels Realtime-Elastographie verbessert in Kombination mit dem bekannten B-Bild die Erfassung der Dignität von Mammaläsionen.

\section{P69}

Interobservervariabilität und Stellenwert der mammographischen Diagnose des invasiv lobulären Mammakarzinoms

*Anja Weidner ${ }^{1,2}$, Karin Bock ${ }^{1,2}$, Volker Duda1,2, Henning Niebuhr2, Annette Ramaswamy ${ }^{3}$, Mark Kalinowski ${ }^{1}$, Uwe Wagner ${ }^{1}$ ${ }^{1}$ Senologische Diagnostik / Klinikum der Philipps-Universität Marburg, Marburg, Deutschland

${ }^{2}$ Qualitätszirkel Mammadiagnostik Mittelhessen, Deutschland

${ }^{3}$ Institut für Pathologie / Klinikum der Philipps-Universität Marburg Marburg, Deutschland

Fragestellung: 10-20\% aller Mammakarzinome sind invasiv lobulär. Aufgrund des lobulären Wachstums zeigen diese besondere Charakteristika in der bildgebenden Diagnostik. Die Früherkennung kann dadurch erheblich eingeschränkt werden. Wie hoch ist die Treffsicherheit der Mammographie in der Evaluation durch Experten eines Qualitätszirkels? Gib es mammographische Charakteristika lobulärer Karzinome, die besonders häufig übersehen werden? Material und Methode: 11 Mitglieder des Qualitätszirkels Mammadiagnostik Mittelhessen werteten anonymisiert unter standardisierten Bedingungen 23 sekundär digitalisierte $(300$ dpi) Mammographiepaare in 2 Standardebenen nach dem BI-RADSys tem (3rd ed.) aus: darunter 20 histologisch gesicherte invasiv lobuläre Karzinome und 6 benigne Herdbefunde. Die Karzinomprävalenz war den Untersuchern nicht vorher bekannt. Ergebnisse: Bezogen auf den Einzeluntersucher lag die mittlere Sensitivität der Karzinomdiagnose bei $83 \%$ (Range 74\%-95\%), die Spezifität bei $86 \%$ (Range $67 \%-96 \%$ ) und die Treffsicherheit bei 78\% (Range 53\%-89\%). Besonders häufig fehlinterpretiert wurden folgende Charakteristika: Asymmetrie und Darstellbar- keit der Befunde in nur einer Ebene. Schlussfolgerung: Die Treffsicherheit der Mammographie ist beim invasiv lobulären Karzinom eingeschränkt. Kenntnis der speziellen Charakteristika lobulärer Karzinome kann die mammographische Diagnosestellung begünstigen.

\section{P70}

\section{MR-Elastographie (MRE) zur Analyse von benignen und} malignen Mammaveränderungen

\author{
*Tanja Xydeas ${ }^{1}$, Katja Siegmann ${ }^{1}$, Ralph Sinkus ${ }^{2}$ \\ ${ }^{1}$ Radiologische Diagnostik, Universitätsklinikum Tübingen, \\ Tübingen, Deutschland \\ ${ }^{2}$ Philips Forschung, Hamburg, Deutschland
}

Einleitung: Die MR-Mammographie ist ein unverzichtbarer Bestandteil der modernen Mammadiagnostik. Bei hoher Sensitivität führt die mäßige Spezifität des Verfahrens jedoch zu einer relevanten Anzahl falsch positiver Läsionen. Die MR-Elastographie könnte als zusätzliches objektives Verfahren helfen, diese zu verringern. Zielsetzung: Erhöhung der Spezifität der MR-Mammographie mittels additiver Elastographie zur besseren Unterscheidung von benignen und malignen Mammaläsionen. Material und Methode: Wir haben 20 Patientinnen mit benignen $(\mathrm{n}=11)$, malignen $(\mathrm{n}=5)$ oder ohne fokale Läsionen $(\mathrm{n}=4)$ bei 1,5 Tesla (Philips Gyroscan Intera) untersucht. Nach einer kontrastangehobenen dynamischen MRMammographie beider Mammae wurden einseitig mechanische Wellen $(65 \mathrm{~Hz})$ mittels Oszillator (Elastographiespule, Philips Medizin Systeme, Hamburg) gezielt in die zu untersuchenden Gewebsschichten ( $\mathrm{n}=7$ á 2,5 mm Dicke) eingekoppelt. Die Vibration des angeregten Gewebes wurde mittels einer bewegungssensitiven Spinechosequenz gemessen und daraus eine lokale Elastizitätsverteilung rekonstruiert. Ergebnisse: Technisch konnten alle Untersuchungen bei einer mittleren Untersuchungszeit von 25 Minuten durchgeführt werden. Die Befunde konnten in der MRE mit den kontrastverstärkten Bildern der dynamischen Sequenzen korreliert werden. Dabei zeigten die Malignome höhere Elastizitätswerte als benigne Läsionen und das umgebende Brustdrüsengewebe. Zusammenfassung: Die vorliegende Pilotstudie zeigt, dass maligne und benigne Läsionen unterschiedliche elastische Eigenschaften aufweisen. Dies muß durch ein größeres Studienkollektiv validiert werden.

\section{Poster Prognosefaktoren/Molekularbiologie}

\section{P7}

Vergleich der Steroidhormonrezeptoren, des HER2/neu-Status und der Proliferation bei Mammakarzinom mit und ohne begleitende in-situ-Komponente

*Mayada R. Bani', Peter A. Fasching1, Michael P. Lux ${ }^{1}$, Christian Löhberg ${ }^{1}$, Thomas Koschek ${ }^{2}$, Thomas Papadopoulos ${ }^{2}$ Matthias W. Beckmann ${ }^{1}$

${ }^{1}$ Unifrauenklinik, Erlangen, Deutschland

${ }^{2}$ Institut für Pathologie, Erlangen, Deutschland

Zielsetzung: Inadäquat behandelte in-situ-Karzinome der Brust können bei Progress zu invasiven Karzinomen werden. Bekannt ist, dass verschiedene histopathologische Kriterien in Abhängigkeit vom Grading beim insitu-Karzinom differieren. In dieser Untersuchung soll geklärt werden, ob histopathologische Parameter bei invasivem Mammakarzinom (MaCa) mit begleitender in-situ-Komponente (isK) ähnliche Abhängigkeiten zeigen. Material und Methode: Anhand von 632 Patientinnen mit invasivem $\mathrm{MaCa}$ wurden mittels odd ratios die Assoziation zwischen dem Östrogen (ER)- und Progesteronrezeptorstatus (PR) und dem HER2/neu-Status und der Proliferation anhand MIB-1 und dem Grading bestimmt. In einer multivariaten Analyse wurde die Unabhängigkeit der Parameter überprüft. Ergebnisse: Für MaCa mit begleitender DCIS-Komponente wurde für den positiven HER2-Status eine OR von 2,103 und für MaCa mit LCIS-Komponente eine positive Assoziation mit positivem ER und positivem PR und eine negative Assoziation mit hoher Proliferation, dem HER2-Status und dem Grading gesehen, während MaCa ohne isK keine Assoziation zeigten. In der multivariaten Analyse zeigte sich die begleitende DCIS-Komponente unabhängig vom Grading als Indikator für 
einen positiven HER2-Status. Zusammenfassung: Im Vergleich mit Karzi nomen ohne isK zeigen mit DCIS-assoziierte-MaCa einen häufiger positiven HER2-Status, LCIS-assoziierte MaCa häufiger eine positiven ER/PR Status und seltener eine hohe Proliferation oder einen postitven HER2 Status. Hieraus könnten Schlussfolgerungen zum invasiven Charakter von high-grade-DCIS gezogen werden.

\section{P72}

\section{Her2-Expression auf disseminierten Tumorzellen beim Mammakarzinom}

*Sven Becker, Tanja Fehm, Graziella Becker-Pergola, Jens Huober, Babur Aydeniz, Diethelm Wallwiener, Erich-Franz Solomayer Universitätsfrauenklinik Tübingen, Tübingen, Deutschland

Hintergrund: Der Nachweis Zytokeratin-positiver $(\mathrm{CK}+)$ Zellen im Knochenmark (KM) von Mammakarzinom-Patientinnen ist als etablierter Prognosefaktor bisher noch ohne therapeutische Konsequenz. Uns interessierte die Rate Her2-positiver Zellen bei CK+ Patientinnen als potientieller therapeutischer Ansatz. Methode: Bei 105 Patientinnen mit nachgewiesenen CK+ Zellen im KM wurde eine zusätzliche immunzytochemische Her2-Färbung bzw. eine RTPCR-Analyse auf Her2 durchgeführt. Die Ergebnisse wurden mit dem Tumostadium sowie dem Her2-Status des Primärtumors verglichen. Ergebnis: Bei 22/105 Fällen fanden sich Her2-positive Zellen (21\%). Die RTPCR- Technik detektierte in 15\% Her2 im KM. Die Her2-Überexpression im Primärtumor betrug 26/105 (25\%). Die Korrelation mit dem KM-Status war wie folgt: Hercep-Score 0/1+: 79 Patientinnen mit 10 für Her2-positiven KM (12\%). Hercep-Score 2+/3+: 26 Patientinnen mit 12 für her2 positiven KM $(46 \%, p=0,001)$ Fazit: Wir fanden Her2-positive Zellen in 15-21\% der MamamkarzinomPatientinnen mit nachgewiesenen CK+ Zellen im KM. Diese Zahl ist niedriger als in den existierenden Studien zu dieser Fragestellung. Eine Korrelation bestand zu Präsenz von Her2 auf dem Primärtumor sowie zum Ausmaß der Positivität gemäß dem Herceps-Score. Trotz dieser Korrelation fanden wir bei $12,6 \%$ von Patientinnen mit Her2 negativem Primärtumor Her2-positive Zellen im KM.

\section{P73}

\section{Präsenz zytokeratin-positiver Zellen im Knochenmark von Mammakarzinom-Patientinnen nach präoperativer systemischer Therapie}

*Sven Becker, Tanja Fehm, Graziella Pergola, Babur Aydeniz, Jens Huober, Diethelm Wallwiener, Erich Solomayer

Universitätsfrauenklinik, Tübingen, Deutschland

Hintergrund: Zytokeratin-(CK)-positive Zellen im Knochenmark (KM) von Mammakarzinompatientinnen sind ein unabhängiger Prognosemarker. Zytogenetische Studien unterstützen die Theorie, dass es sich um mikrodisseminierte Tumorzellen handelt. Der Effekt der adjuvanten und primären systemischen Therapie (PST) auf diese Zellen ist von besonderem Interesse vor dem Hintergrund einer persistierend hohen Rate von Spät-Metastasierung bei optimal behandelten Hochrisiko-Patientinnen, die klinisch sonst völlig tumorfrei waren. Methode: 70 Patientinnen, die primär systemisch behandelt waren, wurden zum Zeitpunkt der Operation knochenmarkspunktiert. 58 Patientinnen erhielten primäre Chemotherapie, 12 erhielten primäre Hormontherapie. Die Präsenz zytokeratinpositiver Zellen im Knochenmark wurde mit Immunzytochemie (A45BB3 Antikörper) sowie der APAAP-Detektionstechnik untersucht. Ergebnis: Durchschnittlichsalter war 54 Jahre. Klinischer, prätherapeutischer T-Status war: T4 $=25$ Pat., T3 $=13$ Pat., T1/2 $=32$ Pat. Zum Zeitpunkt der Operation hatten 33 Pat. (47\%) CK-positive Zellen im Knochenmark. Die Tumor-Response verhielt sich zur Präsenz von CK-positiven Zellen wie folgt: 14 Pat. (20\%) mit kompletter Remission davon 6 mit CK-positiven Zellen im KM (CK-Positivität 42\%). 34 Pat. (49\%) mit partieller Remission, davon 14 mit CK-positiven Zellen (41\%). 21 Pat. (31\%) ohne jegliches Tumoransprechen, davon 12 mit CK-positiven Zellen $(57 \%)$. Der Vergleich primäre Chemotherapie vs. primäre Hormontherapie zeigte bei 29/58 (50\%) Patientinnen (Chemotherapie) sowie bei 4/13 (33\%) (Hormontherapie) CK-positive Zellen im KM. Fazit: Nach Abschluß der PST finden sich bei fast $50 \%$ der Patientinnen CK-positive
Zellen im Knochenmark. Die Zahlen sind vergleichbar einem klinisch nach TNM-Stadium ähnlichen Kollektiv vor systemischer Therapie. MÖglicherweise werden die CK-positiven Zellen im KM durch die PST nicht adäquat eliminiert oder der Tumor erneuert ihre Präsenz durch kontinuierliches Shedding während der Therapie.

\section{P74}

\section{Nachweis okkulter Tumorzellen im Knochenmark von Mammakarzinom-Patientinnen nach adjuvanter Therapie}

*Sven Becker, Graziella Pergola, Tanja Fehm, Babur Aydeniz, Jens Huober, Diethelm Wallwiener, Erich Solomayer Universitätsfrauenklinik, Tübingen, Deutschland

Hintergrund: Wir betrachten das Schicksal von okkulten Tumorzellen (TZ) im Knochenmark (KM), ein unabhängiger Prognosefaktor beim Mammakarzinom, unter adjuvanter Therapie. Methode: Der Nachweis der TZ erfolgte zum Zeitpunkt der Primäroperation sowie nach Ende der adjuvanten Therapie durch KM-Punktion und immuncytochemische Färbung auf zytokeratin-positive $(\mathrm{CK}+)$ Zellen. Ergebnis: In einem Hochrisikokollektiv von 98 Patientinnen konnten zum Zeitpunkt der OP bei 76 (78\%) CK+ TZ nachgewiesen werden. Nach adjuvanter Therapie bei 22 (22\%) Patientinnen. 30 der Patientinnen erhielten adjuvante Chemotherapie $(\mathrm{TZ} \mathrm{im} \mathrm{KM}$ vor $\mathrm{CT}=76 \%$, nach $\mathrm{CT}=43 \%), 26$ erhielten adjuvante Hormontherapie ( $\mathrm{TZ}$ im KM vor $\mathrm{HT}=92 \%$, nach $\mathrm{HT}=15 \%$ ), 42 erhielten eine Kombination aus CT und HT (TZ im KM vor Kombinationstherapie $69 \%$, nach Therapie $26 \%$ ). Schlussfolgerung: Adjuvante systemische Therapie reduziert die Inzidenz CK+ Zellen im Knochenmark. Trotzdem verbleibt eine Gruppe von $22 \%$, bei denen auch nach Abschluß der Therapie potentiell mikrodisseminierte TZ im KM vorliegen. Interssanterweise lag die Zahl bei rein hormoneller Adjuvanz am niedrigsten.

\section{P75}

57 Monate überleben trotz hochgradiger Thrombopenie infolge primärer Knochenmarkskarzinose eines Mammakarzinoms

*Vesna Bjelic-Radisic, Edgar Petru

Geburtshilflich-gynäkologische Univ. Klinik Graz,

Abteilung für allgem. Gynäkologie, Graz, Österreich

$7-10 \%$ aller Patientinnen mit Mammakarzinom weisen primär bereits eine Fernmetastasierung auf. Eine Knochenmarkskarzinose mit Thrombopenie zum Zeitpunkt der Erstdiagnose eines Mammakarzinoms ist extrem selten.

Wir beschreiben den Einzelfall einer 62-jährigen Patientin mit primär metastasierendem, exulzeriertem, invasiv duktalem Mammakarzinom, ER/PR+, Her-2+++, Knochenmarkkarzinose, Lungen- und frontoparietalien Metastasen und initialer Thrombopenie von $15000 / \mathrm{mm}^{3}$.

Zum Beginn verabreichten wir eine aggressive Chemotherapie mit Doxorubicin und Docetaxel um durch die zytotoxische Effektivität das Knochenmark zu treffen und damit die Thrombozytenproduktion zu steigern. In weiteren 57 Monaten wegen Nichtansprechens, bzw. Tumorprogression erhielt die Patientin 10 Zyklen Capecitabine, Exemestan, 6 Zyklen CMF, Vinorelbine, 11 Zyklen Gemcitabine,4 Zyklen Carboplatin, Trastuzumab, palliative Sthrahlentherapie des Schädels und des Beckens bei diffuser ossärer Metastasierung. Retrospektiv profitierte die Patientin in Bezug auf ihre Leistungsfähigkeit und klinische Stabilisation durch die Gabe von Capecitabine, Exemestan, Gemcitabine und Trastuzumab. Der höchste bestimmte Wert der Thrombozyten lag bei $148000 / \mathrm{mm}^{3}$, der kurz nach der Initiirung der Trastuzumab -Therapie zu erreichen war. Vielfach lag unter der Chemotherapie der Thrombozytenwert unter 10 000/mm ${ }^{3}$ (Abb. 1), ohne klinische Blutungsmanifestationen. Wegen hochgradiger Thrombopenie $\left(2,000 / \mathrm{mm}^{3}\right)$ wurden zweimal je 2 Thrombozytenkonzentrate verabreicht. CEA und CA-15/3 Werte zeigten keine Korrelation mit dem klinischen Verlauf oder Thrombozytenwerten.

Zusammenfassend zeigt dieser Fallbericht, dass das Überleben einzelner Patientinnen trotz massiver initialer Metastasierung mit Befall des Knochenmarks durch die Anwendung verschiedener Standard-Chemotherapien, antihormoneller Therapien bzw. Therapien mit monoklonalen Antikörpern über fast 5 Jahre ermöglicht werden kann, ohne dass schwere Thrombopenie-bedingte Blutungskomplikationen auftreten. 
P76

\section{Einfluss von Glukokortikoiden auf das Onkogen Her4/Erbb4}

*Ansgar Brüning, Daniela Weiß, Lioba Walz, Elmar Stickeler, Gerald Gitsch, Ingo B. Runnebaum

Frauenklinik, Universität Freiburg, Freiburg, Deutschland

Zielsetzung: Wachstumsfaktorrezeptoren der Tyrosinkinasefamilie, wie EGFR oder Her2 sind häufig überexprimierte und für die Therapie prädiktive Faktoren beim Mammakarzinom. Wir haben den Einfluss von Glukokortikoiden auf Expression und Splicing von Erbb4 in Mammakarzinomzellen untersucht. Materialien und Methoden: MCF7 und T47D Mammakarzinomzellen wurden mit $100 \mathrm{nM}$ Dexamethason oder Cortisol inkubiert. Zellproliferation wurde mittels eines Hämozytometers bestimmt. Die Expression von Erbb4 wurde mittels RT-PCR und Western Blot Analyse untersucht. Das Splicing von Erbb4 in eine Phosphatidylinositol-3kinase bindende Form (CTa) und eine nicht-Phosphatidylinositol-3Kinase bindende Form (CTb) wurde mittels spezifischer PCR-Prime analysiert. Ergebnisse: Dexamethason und Cortisol in einer Konzentration von $100 \mathrm{nM}$ vermindern die Proliferationsrate von MCF7 und T47D Zellen um zirka $15 \%$ nach 4tägiger Inkubation. Glukokortikoidbehandlung erhöht die Gesamtexpression von Erbb4 in MCF7 und T47D Zellen um das 2-3fache. Spezifische RT-PCR Analyse auf unterschiedliche Spliceformen ergab eine verstärkte Expression der CTb Variante ohne Phosphatidylinositol-3kinase Bindungsdomäne. Zusammenfassung: Glukokortikoide erhöhen die Expression der nicht-Phosphatidylinositol-3kinase bindenden Erbb4 Isoform in Mammakarzinomzellen. Glukokortikoide verringern somit die vom Erbb4 ausgehenden zellproliferationsfördernden Signalmechanismen.

\section{P77}

\section{Morphometrischer Nachweis der HER2-Genamplifikation mit chromogener in-situ Hybridisierung (CISH) durch Absorptions- mikroskopie beim Mammacarcinom}

${ }^{*}$ Karl-Friedrich Bürrig ${ }^{1}$, Olaf Ahrens ${ }^{2}$, Christian Ondo-Meva ${ }^{1}$

${ }^{1}$ Institut für Pathologie, Hildesheim, Deutschland

${ }^{2}$ Messtechnische Beratung, Barteheide, Deutschland

Ziel: Der immunhistochemische Nachweis einer schwachen HER2-Überexpression macht nach den z.Zt. gültigen Empfehlungen eine weitere personal- und zeitaufwändige Analyse mit Fluoreszenz in-situ Hybridisierung (FISH) erforderlich. Es sollte ein Algorithmus zur morphometrischen Analyse mittels CISH beim Mammacarcinom (MCA) entwickelt werden. Material und Methodik: 46 MCA mit schwacher HER2-Überexpression (DAKO 2+) wurden analysiert. CISH wurde mit einer Digoxigenin-DNSSonde (Zymed) durchgeführt. Mit dem Ahrens-Image-Cytometry-System (Ahrens ICM) wurden positive CISH-Signale durch Messung optischer Dichten (Absorptionsmikroskopie) nachgewiesen. Dabei wurde positive Signale (spots und cluster) als Elemente einer Pixelmatrix bei Hellfeldmikroskopie mit 20facher oder 40facher Vergrößerung gesucht. Die Kernfärbung erfolge mit Hämatoxylin. Als Kontrolle wurden konventionell morphologisch FISH- und CISH-Präparationen ausgewertet. Ergebnisse: Der morphometrische Algorithmus erlaubt eine sensitive und spezifische Analyse zum Nachweis einer HER2-Genamplifikation, wobei auch große Tumorflächen im MCA analysiert werden können. Tumorzellen und begleitende Zellelemente sind gut unterscheidbar, durch interaktive Korrektur beim Messvorgang können Fehlbestimmungen verhindert werden. Zwischen konventioneller und morhometrischer Analyse ergab sich eine Konkordanz von 89\%. Die ohne Abdunkelung durchführbare CISH-Messung ist weniger zeitaufwändig und personalintensiv als die FISH-Technik. Zusammenfassung: Die morphometrische Analyse des MCA mittels CISH-Technik ist eine schnelle, sensitive und spezifische Methode zum Nachweis einer HER2-Genamplifikation bei immunhistochemisch schwacher Überexpression im MCA.

\section{P78}

Hochdurchsatz-Identifizierung und molekularbiologische Charakterisierung von Genen, die im Mammakarzinom differentiell exprimiert werden

*Edgar Dahl1,7, Ariane Sadr-Nabavi², Eva Klopocki3,7, Beate Betz , Susanne Grube ${ }^{5}$, Rene Kreutzfeld ${ }^{6}$, Marina Himmelfarb ${ }^{1,7}$,

Stephen Gelling ${ }^{7}$, Irina Klaman ${ }^{7}$, Bernd Hinzmann $^{7}$, Glen Kristiansen ${ }^{8}$, Robert Grützmann ${ }^{9}$, Ruprecht Kuner ${ }^{7}$, Beate Petschke ${ }^{7}$, Kerstin Rhiem ${ }^{6}$, Kai Wiechen ${ }^{8}$, Christine Sers ${ }^{8}$, Otmar Wiestler ${ }^{10}$, Achim Schneider ${ }^{5}$, Heinz Höfler ${ }^{11}$, Jörg Nährig ${ }^{11}$, Manfred Dietel ${ }^{8}$, Reinhold Schäfer $^{8}$, Andre Rosenthal 7 , Rita Schmutzler ${ }^{6}$, Matthias Dürst ${ }^{5}$,

Dieter Niederacher ${ }^{4}$, Alfons Meind ${ }^{2}$

${ }^{1}$ Institut für Pathologie, Universitätsklinikum der RWTH Aachen,

Aachen, Deutschland

${ }^{2}$ Kinderklinik, Medizinische Genetik,

Ludwig-Maximiliam Universität, München, Deutschland

IInstitut für Medzinische Genetik, Humboldt Universität Berlin,

Berlin, Deutschland

${ }^{4}$ Molekulargenetisches Labor, Frauenklinik der Heinrich-Heine-

Universität, Düsseldorf, Deutschland

${ }^{5}$ Klinik für Frauenheilkunde und Geburtshilfe,

Friedrich-Schiller-Universität, Jena, Deutschland

${ }^{6}$ Frauenklinik der Universität Köln, Köln, Deutschland

${ }^{7}$ metaGen Pharmaceuticals i. L., Berlin, Berlin, Deutschland

${ }^{8}$ Institut für Pathologie, Humboldt Universität Berlin, Berlin,

Deutschland

${ }^{9}$ VTG Chirugie, Technische Universität Dresden, Dresden,

Deutschland

${ }^{10}$ Institut für Neuropathologie, Universität Bonn, Bonn, Deutschland

${ }^{11}$ Institut für Pathologie, Technische Universität München, München,

Deutschland

Zielsetzung: Im Mammakarzinom differentiell exprimierte Gene stellen einen Fundus von potentiellen Marker- und Targetgenen dar, die mittelfristig zu verbesserten Diagnose- und Therapieverfahren dieser Tumorerkrankungen genutzt werden können. Material und Methoden: Differentiell exprimierte Gene wurden anhand eines bioinformatischen Verfahrens identifiziert, welches die Information von EST («Expressed Sequence Tag")-cDNA Bibliotheken nutzt (Schmitt et al. 1999; Nucl. Acids Res. 27:4251-60). Ausgehend von zunächst 300 im Mammakarzinom differentiell exprimierten Genen wurde nach Kombination von Expressions- Kartierungs- und Funktionsdaten ein Set von 25 besonders interessanten Kandidatengenen definiert. Diese Gene kartieren in chromosomalen Regionen, denen man eine wichtige Rolle bei der Entstehung des Mammakarzinoms zuspricht. Da sie ferner aufgrund ihrer postulierten Funktion an wichtigen zellulären Prozessen beteiligt sein können, stellen sie neue putative Tumorsuppressor- und Onkogene dar. Ergebnisse: Mittels DotBlot-Array Analyse an 50 Normal/Tumor cDNA Paaren des Mammakarzinoms konnten wir die differentielle Expression für $70 \%$ (18/25) der Kandidatengene bestätigen; darunter befinden sich $11 \mathrm{Gene}$, die bisher noch nicht mit Bezug zum Mammakarzinom beschrieben wurden. Einige dieser Gene besitzen Potential als neue diagnostische Marker, so z.B. das putative Tumorsuppressorgen SFRP1 im frühen Mammakarzinom (Klopocki et al.; Int. J. Onc., in press). Die Mutationsanalyse von 10 Kandidatengenen in einem Set von 20 primären Mammakarzinomen, die auf genspezifischen «Loss of heterozygosity» $(\mathrm{LOH})$ selektiert wurden, entdeckte nur in zwei Genen inaktivierenden Mutationen. Zusammenfassung: Der geringe Prozentsatz von Kandidatengenen mit Mutationen deutet daraufhin, dass die Mehrzahl der in gynäkologischen Karzinomen abgeschalteten Gene durch epigenetische Mechanismen oder trans-regulatorische Faktoren inaktiviert wird. Die dargestellte Strategie ist geeignet rasch neue tumor-assoziierte Gene des Mammakarzinoms zu identifizieren. 


\section{P79}

\section{CAR in einem murinen Mammakarzinommodell}

*Daniel Diederich, Elmar Stickeler, Lioba Walz, Ansgar Brüning, Gerald Gitsch, Ingo B. Runnebaum

Frauenklinik, Universität Freiburg, Freiburg, Deutschland

Zielsetzung: Der Coxsackie-Adenovirus Rezeptor (CAR) ist ein Zelladhäsionsprotein in Tumorzellen und kann die Zellmigration und Zelldisseminierung inhibieren (Brüning und Runnebaum, Exp. Cell Res. 2004). Wir untersuchten an einem definiertem Mausmammakarzinommodell die Expression von CAR in verschiedenen Tumorentwicklungsstadien. Materialien und Methoden: Durch serielle Implantation der präneoplastischen Maus-Mammakarzinomzelllinie TM2L in das Maus-Mammafettpolster konnten Gewebezelllinien mit unterschiedlicher Tumorigenität und Metastasierungsneigung gewonnen werden. Aus diesen Geweben wurde RNA isoliert und mittels RT-PCR auf die Expression von CAR untersucht. Ergebnisse: Während im präneoplastischem Mausmammakarzinomgewebe TM2L die Expression von CAR gegenüber dem untransformierten, reifen Mammadrüsengewebe kaum verändert war, fand sich in vielen Tumoren mit geringer Metastasierungsneigung eine erhöhte CAR Expression. Eine deutlich verringerte CAR Expression konnte in der Gewebezelllinie TM40D mit erhöhter Neigung zur Metastasenbildung beobachtet werden. Eine aus diesem TM40D Gewebe resultierende Lebermetastase zeigte ebenfalls eine erniedrigte CAR Expression. Zusammenfassung: Das Zelladhäsionsprotein CAR zeigt eine differentielle Expression in verschiedenen Tumorstadien. CAR ist verringert exprimiert in Mammakarzinomzellen mit erhöhtem Metastasenpotential. Verringerte CAR Expression könnte das Ablösen von Tumorzellen aus dem Zellverband ermöglichen und Metastasierung erleichtern.

\section{P80}

\section{Mamma-Carcinom bei jungen Patientinnen unter 36 Jahren}

*Uta Euler ${ }^{1,3}$, Bianca Knopf ${ }^{1}$, Joke Tio ${ }^{1,4}$, H. Volkholz ${ }^{2}$,

Eva-Maria Grischke ${ }^{3}$, Agustinus H. Tulusan ${ }^{1}$

${ }^{1}$ Frauenklinik Bayreuth, Bayreuth, Deutschland

${ }^{2}$ Abteilung für Pathologie Klinikum Bayreuth, Bayreuth,

Deutschland

${ }^{3}$ Abteilung für Gynäkologie u. Geburtshilfe Krankenhaus München

Schwabing, München, Deutschland

${ }^{4}$ Abteilung für Gynäkologie u. Geburtshilfe Universitätklinik

Münster, Münster, Deutschland

Zielsetzung: Mamma-Carcinom bei jungen Patientinnen ist häufig assoziiert mit aggressiven Tumoreigenschaften und schlechter Prognose. In einer retrospektiven Analyse wurden pathologische und biologische Fak toren bei jungen Patientinnen untersucht. Methoden: 56 Patientinnen mit Mamma-Carcinom unter 36 Jahren wurden analysiert. Mittleres Alter: 31,5 Jahre. 26 Patientinnen erhielten neoadjuvante, 26 Patientinnen adjuvante Chemotherapie. 46 konnten brusterhaltend operiert werden, bei 7 wurde die OP mit einer onkoplastischen kombiniert. 6 Patientinnen erhielten CMF. 26 wurden mit anthrazyklinhaltigen, 8 mit taxanhaltigen Chemotherapie, 5 mit einer Kombination behandelt. Fast alle (96\%) wurden bestrahlt. Tumorgewebe wurde nach der OP auf ER, PgR, p53, Ki67, Her2-neu, EGRF, UPA und PAI1 untersucht. Ergebnisse: In 79\% war de Primärtumor $>2 \mathrm{~cm}$. Alle Patientinnen mit T3 und T4 Tumoren wurden neoadjuvant behandelt. Bei $48 \%$ waren die Lymphknoten befallen. $80 \%$ aller Tumoren waren ductal-invasiv, 59\% wurden als G3 klassifiziert. 67\% waren ER positiv, 55\% PgR positiv. p53 war in $41 \%(\mathrm{n}=37)$ mutiert Her2-neu in $40 \%(n=47)$, UPA in $85 \%(n=27)$, PAI1 in $93 \%(n=27)$ positiv, erhöhtes Ki67(>18\%) $(n=38)$ in $73 \%$. EGRF war in $84 \%(n=26)$ negativ. Bei positivem EGRF $(\mathrm{p}=0,04)$ oder positivem Her2-neu ( $\mathrm{p}=$ $0,02)$ kommen Metastasen häufiger vor. Keine erhöhte Rate an Lokalrezidiven bei brusterhaltend operierten Patientinnen. $87 \%$ sind rezidivfrei 71\% metastasenfrei, 83\% leben noch. Mittleres Follow-up: 44 Monate. Schlussfolgerung: Trotz aggressiverer und ungünstigerer Tumoren konnten $82 \%$ ohne ein erhöhtes Lokalrezidivrisiko brusterhaltend operiert werden. Bei positivem EGRF oder Her2-neu Status treten mehr Metastasen auf. Mehr als $80 \%$ der Patientinnen sind noch am Leben. Eine individuelle Anpassung der Behandlung jeder einzelnen Patientin scheint notwendig und wichtig.

\section{P81}

\section{HER-2/neu Gen Amplifikation und Protein Expression in männlichen Mammakarzinomen}

*Nicolaus Friedrichs, Christian Rudlowski ${ }^{1}$, Andree Faridi ${ }^{2}$ Lazlo Fuzesi ${ }^{3}$, Roland Moll ${ }^{4}$, Werner Rath ${ }^{2}$, Reinhard Buettner Institut für Pathologie, Universität Bonn, Bonn, Deutschland, ${ }^{1}$ Frauenklinik, Universität Heidelberg, Heidelberg, Deutschland ${ }^{2}$ Frauenklinik, RWTH Aachen, Aachen, Deutschland, ${ }^{3}$ Institut für Pathologie, Universität Göttingen, Göttingen, Deutschland

${ }^{4}$ Institut für Pathologie, Universität Marburg, Marburg, Deutschland

Ziele: Die Häufigkeit von HER-2/neu Überexpression und Amplifikation in humanen männlichen Mamma-Karzinomen sollte untersucht werden. Material und Methoden: In 99 männlichen Brustkrebsfällen wurde die HER2 Protein Expression mittels DAKO HercepTest ${ }^{\circledR}$ und die HER2 Genkopienanzahl durch Fluoreszenz-in-situ-Hybridisierung (FISH) mittels Vysis PathVysion Test ${ }^{\circledR}$ untersucht. Ergebnisse: Eine HER2 Protein Überexpression wurde in 15,1\% festgestellt (15/99 Fälle). Eine HER2 Amplifikation wurde in 11,1\% nachgewiesen (11/99 Fälle); dies zumeist als High-Level Amplifikation. Unter diesen amplifizierten Tumoren wurden 8 Fälle immunhistochemisch als $2+$ und 7 Fälle als $3+$ bewertet. Alle $3+$ Fälle zeigten HER2 Amplifikation in der FISH und 4 von 8 als $2+$ bewertete Fälle waren ebenfalls amplifiziert. In statistischen Analysen korrelierten HER2 Überexpression oder Amplifikation nicht mit anderen diagnostischen Parametern wie Malignitätsgrad oder Hormonrezeptorstatus. Zusammenfassung: Dies ist die erste systematisierte Studie über HER2 Amplifikation mittels FISH Analysen in männlichem Brustkrebs. In der vorliegenden Studie war die Rate der HER2 Überexpression $(15,1 \%)$ niedriger als in weiblichen Brustkrebsfällen $(20 \%) .11,1 \%$ der Fälle zeigten eine Amplifikation des HER2 Gens. Das Vorhandensein von HercepTest-positiven wie auch FISH-amplifizierten Fällen in diesen 99 männlichen Brustkrebsfällen lässt vermuten, dass eine Therapie mit dem monoklonalen HER2/neu Antikörper (HERCEPTIN ${ }^{\circledR}$ ) auch in der Behandlung von männlichem Brustkrebs anwendbar wäre.

P82

\section{Verringerte Expression von TCR $\zeta$-Ketten und CD28 auf peripheren T-Zellen von Patientinnen mit primären} Mammakarzinom - Hinweis für eine gestörte T-Zellaktivierung

I. Gruber*, D. Wallwiener, T. Fehm, S. Dürr-Störzer, G. Becker-Pergola, E. F. Solomayer

Universitätsfrauenklinik, Tübingen, Deutschland (Ärztlicher Direktor: Prof. Dr. D. Wallwiener)

Zielsetzung: Eine tumorspezifische Immunantwort fordert die klonale Expansion und Differenzierung naiver T-Zellen zu bewaffneten T-Effektorzellen. Hierbei ist die Bindung zwischen MHC-Peptid-Komplex und T-Zellrezeptor (TCR), einhergehend mit einer effektiven Signaltransduction durch TCR $\zeta$-Ketten, entscheidend. Zusätzlich benötigt die Aktivierung naiver T-Zellen costimuliernde Signale, welche durch die Bindung von CD28 der T-Zellen an die B7-Moleküle der antigenpräsentierenden Zelle freigesetzt werden. Um nun die funktionelle Integrität der spezifischen Immunantwort beim Mammakarzinom zu überprüfen, untersuchten wir die Expression von TCR $\xi$ und CD28 auf peripheren T-Zellen. Methode: Anhand der Durchflußzytometrie wurde das Blut von 64 Patientinnen mit primären Mammakarzinom (charakterisiert nach klinisch-pathologischen Parametern) im Vergleich zu gesunden Probandinnen $(\mathrm{n}=28)$ analysiert. Hierbei wird die quantitative Expression von TCR $\zeta$-Ketten und CD28 auf CD8+ bzw. CD4+T-Lymphozyten erfaßt. Ergebnisse: Der quantitativen Vergleich von $\mathrm{CD} 8+/ \mathrm{TCR} \zeta+$ bzw.

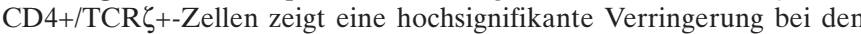
Mammakarzinompatientinnen $(\mathrm{p}<0,001)$. Außerdem haben die Patientinnen auch signifikant weniger CD28 exprimierende T-Lymphozyten im Blut ( $<<0,012 ; \mathrm{p}<0,004)$. Damit sind die für die T-Zellaktivierung so wichtigen Signalmoleküle bei Mammakarzinompatientinnen deutlich verringert, was einen bedeutenden Einfluß auf die T-zellspezifische Immunantwort haben könnte 
P83

Signifikante Veränderungen des zellulären Immunstatus bei Patientinnen mit primären Mammakarzinom im Vergleich zu Gesunden

I. Gruber*, D. Wallwiener, T. Fehm, S. Dürr-Störzer, G. Becker-Pergola E. F. Solomayer

Universitätsfrauenklinik, Tübingen, Deutschland (Ärztlicher Direktor: Prof. Dr. D. Wallwiener)

Zielsetzung: Erst kürzlich konnte gezeigt werden, daß bei der Immunüberwachung (immune surveillance) nicht nur die lokale Immunabwehr gegen Tumorzellen, sondern auch die Abwehr im Blut eine entscheidende Rolle spielt. Diesbezüglich interessierte uns die zelluläre Zusammensetzung des peripheren Immunstatus von Patientinnen mit Mammakarzinom im Vergleich zu gesunden Probandinnen. Methode: 64 Patientinnen mit primären Mammakarzinom wurden nach Alter, TNM-Status, Histologie Grading und Steroidhormonrezeptor-Status charakterisiert.T-Zellen, NKZellen und B-Zellen aus dem peripheren Blut dieser Patientinnen wurden hinsichtlich Quantität und Aktivitätsstatus untersucht und mit den Ergebnissen gesunder Probandinnen $(n=28)$ verglichen. Die phänotypische Charakterisierung erfolgt per Durchflußzytometrie. Ergebnisse: Die Mammakarzinompatientinnen zeigten signifikant weniger aktivierte zytotoxische T-Zellen und T-Helferzellen. Auch war der Anteil an naiven CD8+ bzw. CD4+-Zellen deutlich verringert. Die Patientinnen hatten außerdem signifikant mehr T-Gedächtniszellen und B-Zellen. Bezüglich NK-Zellen konnten keine signifikanten Unterschiede festgestellt werden. Zwischen zellulärer Immunität und klinisch-pathologischen Prognosefaktoren gabe es ebenfalls keine Signifikanz. Zusammenfassung: Es konnte gezeigt werden, daß bei Patientinnen mit Mammakarzinom im Vergleich zu gesunden Probandinnen Quantität und Aktivitätsstatus von peripheren T-Zellen signifikant verändert sind.

\section{P84}

\section{Das zytotoxische GnRH-Analogon AN-152 induziert} MDR-1-unabhängig Apoptose in Mammakarzinomzellen

*Andreas R. Günthert, Günter Emons

Universitätsfrauenklinik Göttingen, Göttingen, Deutschland

Über 50\% aller Mammakarzinome exprimieren Rezeptoren für GnRH Die Expression dieser Rezeptoren kann für die zielgerichtete zytotoxische Therapie genutzt werden, z. B. mit AN-152, in welchem eine kovalente Bindung von Doxorubicin an [D-Lys6]GnRH vorliegt. Es wurde die Wirksamkeit von AN-152 mit dem des Doxorubicin in Mammakarzinomzellen in vitro verglichen. Weiterhin wurde der Einfluss auf die Expres sion des MDR-1-Genproduktes Pgp untersucht

In den Zelllinien MCF-7, T47D und HCC70 wurde eine dichte Oberflächenexpression von GnRH-Rezeptoren nachgewiesen, in ZR-75-1 zeigte sich eine geringe Oberflächenexpression. In Zellen mit hoher GnRH-R Expressionsdichte zeigte sich eine deutlich erhöhte Apoptoserate induziert durch AN-152 im Vergleich zu Doxorubicin bei gleicher Dosis, insbesondere in Medium ohne FCS. AN-152 wird durch die Serum-Carboxylesterase in GnRH und Doxorubicin gespalten, DFP blockiert diese Spaltung. In Versuchen mit DFP im Medium mit FCS, in welchem die Serum-Carboxylesterase aktiv ist, konnte die Effektivität des AN-152 gesteigert werden. AN-152 und Doxorubicin induzierten in Medium mi FCS in den Zelllinien MCF-7, T47D und HCC70 die Expression des Pgp, AN-152 jedoch in geringerem Ausmaß. Bei Zusatz von DFP konnte die AN-152-induzierte Pgp-Expression aufgehoben werden. In Medium ohne FCS zeigte sich lediglich bei der Behandlung mit Doxorubicin eine gesteigerte Pgp-Expression, jedoch nicht bei AN-152.

AN-152 induziert durch rezeptor-gebundene Internalisierung Apoptose in Mammakarzinomzellen. Die Effektivität ist bei gleicher Dosierung höher als die des Doxorubicins, insbesondere bei nicht vorhandener ode inhibierter Serum-Carboxylesterase. Durch die rezeptorgebundene Internalisierung des AN-152 kann das MDR-1-System, welches durch das lipophile Doxorubicin induziert wird, umgangen werden und eventuell eine primäre oder sekundäre Chemoresistenz umgangen werden.
P85

Prognostische Bedeutung von AgNOR-Proliferationsindizes bei nodalnegativen pT1-Mammakarzinomen

${ }^{*}$ Hans Guski ${ }^{1}$, Klaus-Jürgen Winzer ${ }^{2}$, Eva Barten ${ }^{1}$, Peter Hufnagl ${ }^{1}$, Glen Kristiansen ${ }^{1}$

${ }^{1}$ Institut für Pathologie, Charité, Universitätsmedizin, Berlin,

Deutschland

${ }^{2}$ Klinik für Allgemein-, Viszeral-, Gefäß- und Thoraxchirurgie,

Charité, Universitätsmedizin, Berlin, Deutschland

Zielstellung: Frühzeitige Erkennung des Karzinomrezidiv- und Sterberisikos bei Patienten mit einem nodalnegativen Mammakarzinom im Stadium pT1 und Abgrenzung verschiedener Risikogruppen. Material und Methode: Morphometrische Studie an 83 Patienten (33-82 Jahre, medianes Alter 62 Jahre), die 1991-96 in der Chirurgischen Klinik der Charité Berlin wegen eines invasiven Mammakarzinoms operiert wurden. Bei allen Fällen handelte es sich um ein Tumorstadium pT1 pN0. Mediane Verlaufsbeobachtung 50 Monate (bis 100 Monate). Quantifizierung von AgNOR-Merkmalen an histologischen Schnitten mit immunhistochemischer Doppelfärbung (MIB1/AgNOR und ER/AgNOR)) mittels automatischer Bildanalyse. AgNOR-Vermessung an je 100 MIB1-positiven und MIB1-negativen sowie ER-positiven und ER-negativen Tumorzellen desselben Tumors und Berechnung von AgNOR-Indizes (AgNOR-Parameter $\times$ mittlerer MIB1-Index). Ergebnisse: Die AgNOR-Proliferationsindizes (Parea, Pcount) sind in den Fällen mit schlechter Prognose signifikant höher (Parea 332,7 \pm 286,0; Pcount 112,5 \pm 105,0) als in der Patientengruppe mit besserer Prognose (Parea 153,3 \pm 249,8; Pcount 55,3 $\pm 65,9$ ). ER-negative Tumorzellen weisen höhere AgNOR-Indizes auf als ER-positive Tumorzellen. Die Fälle mit hoher Wachstumsfraktion und höheren AgNOR-Indizes sind mit einem früheren und häufigeren Auftreten von Ereignissen im Krankheitsverlauf (Karzinomrezidiv, Metastasen, Tod) assoziiert. Schlussfolgerungen: Tumorwachstumsfraktion (MIB1-Index) und AgNOR-Merkmale beschreiben mit der Erfassung des Anteils proliferierender Tumorzellen und der Zellproliferationsgeschwindigkeit unterschiedliche tumorbiologische Eigenschaften. Durch die Kombination beider Parameter lassen sich bei Patienten mit kleinen nodalnegativen Karzinomen Fälle mit guter Prognose von solchen mit ungünstiger Prognose abgrenzen.

\section{P86}

\section{Urokinase (uPA) aktiviert die Proliferation von Mammakarzinomzellen}

*Ralf Hildenbrand, Mukesh Gandhari, Uwe Bleyl, Norbert Arens Pathologisches Institut, Universitätsklinikum Mannheim, Mannheim, Deutschland

Das Urokinase-System spielt eine bedeutende Rolle bei der Tumorprogression durch Proteolyse und Unterstützung von Adhäsion, Migration, Zelldissoziation, Angiogenese und Metastasierung. Ziel dieser Studie war es die uPA-abhängige Proliferation von Mammakarzinomzellen zu untersuchen und zu überprüfen, ob die Signaltransduktion über den Urokinaserezeptor (uPAR) erfolgt. Material und Methoden: Konstitutiv uPA-exprimierende MDA-MB-231-Mammakarzinomzellen wurden konventionell und mittels der siRNA-Methode transfiziert. Die uPA-Expressionsabnahme wurde mittels ELISA, FACS, Western Blotting überprüft. Die uPA Expression wurde in den siRNA-transfizierten Zellen auf mRNA Ebene anhand der real-time PCR quantifiziert. Die Proliferation wurde mittels Proliferationsassay, Ki67, FACS (S-Phase) und Zellzählung untersucht. Ferner wurden die uPA-supprimierten Zellen mit HMW-uPA, LMW-uPA, ATF, anti-uPAR IIIF10, anti-uPAR IID7, suPAR, PI-PLC und SB203580 inkubiert. Ergebnisse: Die uPA-Expression wurde sowohl mithilfe der konventionellen Transfektion als auch anhand der siRNATransfektion im Vergleich zu den nativen MDA-MB-231-Zellen signifikant reduziert (60-70\%). Mit dieser uPA-Expressionsabnahme ging eine signifikante Proliferationsabnahme der Karzinomzellen um 25-30\% einher. Die mittels siRNA transfizierten Zellen zeigten auch in der real-time PCR eine deutlich geringere uPA-mRNA Expression. Die Inkubation der transfizierten Zellen mit HMW-uPA und ATF ergab eine signifikante Proliferationszunahme im Vergleich zu denjenigen Zellen, die mit LMWuPA inkubiert wurden. Die Blockierung des uPAR mittels IIIF10 führte 
zu einer signifikanten Abnahme des proliferativen Effekts. GleichermaBen war dies zu beobachten, wenn die Urokinaserezeptoren zuvor mittels PI-PLC abgespalten wurden. Wurde die Mitogen-aktivierende Protein Kinase (MAPK) mittels SB 203580 inhibiert, war der Proliferationsreiz von uPA ebenfalls signifikant eingeschränkt. Zusammenfassung: Uroki nase aktiviert die Karzinomzell-Proliferation über Bindung an den Urokinaserezeptor. Die Signaltransduktion erfolgt möglicherweise über benachbarte Integrine, die die MAP-Kinase aktivieren.

P87

\section{Effekt eine Langzeitbehandlung mit Tamoxifen in vitro auf die Rezeptorexpression und Apoptose von MCF-7 Mammakarzinomzellen}

*Felicitas Horn, Oliver Treeck, Olaf Ortmann

Klinik für Frauenheilkunde und Geburtshilfe, Universität Regensburg, Caritas Krankenhause St. Josef, Regensburg, Deutschland

Zielsetzung: In dieser Studie untersuchten wir den Effekt einer Langzeit Behandlung mit Tamoxifen (TAM) in vitro auf die Genexpression und apoptotische Antwort von MCF-7 Mammakarzinomzellen. Material und Methoden: Wir benutzen einen in vitro Selektionsansatz zur Gewinnung von MCF-7 Zellen, die eine vermindertes Ansprechen auf TAM aufwie sen. Die apoptotische Wirkung von Tamoxifen wurde durch ELISA Messung cytoplasmatischer Histon-DNA Komplexe nachgewiesen. Ergebnisse: MCF-7(LT) Zellen, die nach 5 Monaten Langzeitbehandlung mit Tamoxifen isoliert wurden, wiesen eine signifikant verminderte apoptotische Reaktion auf TAM auf, die bis zu Konzentrationen von $20 \mu \mathrm{M}$ beobachtet werden konnte. In diesen Zellen war auch Etoposid nicht in der Lage, die zelluläre Apoptose zu induzieren. Microarray Experimente, mit denen das Transkriptom der MCF-7(LT) Klone mit dem von Kontrollzellen verglichen wurde, zeigten eine signifikant verstärkte Expression von anti-apoptotischen Genen und die abgeschwächte Expression von Apoptose-Genen. Western Blot Experimente zur Untersuchung des Rezeptorstatus dieser Zellen zeigten keine veränderte Expression der Rezeptoren HER2, EGFR und ER $\alpha$, aber eine verminderte Expression von ER $\beta$ Subtypen. Zusammenfassung: Unsere Daten zeigen, dass eine Langzeit behandlung von MCF-7 Zellen mit Tamoxifen in vitro nicht notwendigerweise die Expression von Rezeptortyrosinkinasen wie HER2 und EGFR beeinflusst. Die langzeitbehandelten MCF-7 Zellen wiesen jedoch eine modulierte Expression von Schlüsselgenen der Apoptose auf, die sich in einer verminderten Apoptose dieser Zellen äusserte.

P88

Epidermale Wachstumsfaktorrezeptoren und deren Liganden beim Mammakarzinom: Her-2/neu als bevorzugter Dimerisationspartner in nodal-positiven Tumoren

${ }^{*}$ Gernot Hudelist ${ }^{1,2}$, Christian Singer ${ }^{1}$, Mahmood Manavi ${ }^{1}$, Kerstin Pischinger ${ }^{3}$, Ernst Kubista ${ }^{1}$, Klaus Czerwenka ${ }^{3}$ ${ }^{1}$ Universitätsfrauenklinik, Abteilung für Spezielle Gynäkologie, Wien, Österreich

${ }^{2}$ Abteilung für Gynäkologie und Geburtshilfe, LKH Villach, Villach, Österreich

${ }^{3}$ Abteilung für Klinische Pathologie, Gynäkopathologie, Wien, Österreich

Problemstellung: Die epidermalen Wachstumsfaktoren EGFR, Her 2/neu, Her-3 und Her-4 werden von zahlreichen Mammakarzinomen exprimiert, wobei Amplifikation/Überexpression von Her-2/neu mit einer Verschlechterung der Prognose einhergeht und einen prädiktiven Stellen wert für das Ergebnis adjuvanter und palliativer therapeutischer Interventionen besitzt. Die Bindung von externen Liganden wie epidermal growth factor (EGF), transforming growth factor beta (TGF-b) oder heregulin (HRG) sowie die Bildung von Rezeptor-Heterodimeren führt zur Aktivierung der mitogenen Signaltransduktionskaskade, welche mit einer entsprechenden Stimulation der Tumorproliferation einhergeht. Zahlreiche in vitro Studien belegen, dass biologisches Verhalten und Tumorwachstum wesentlich von der Zusammensetzung der Rezeptor/Rezeptor Komplexe sowie den vorhandenen Liganden beeinflusst wird. Ziel dieser Studie war es, das Ko-Expressionsprofil aller vier ErbB-Rezeptoren und ihrer wirksamsten Liganden EGF, TGF-a und HRG bei Mammakarzinompatientinnen zu untersuchen. Material und Methodik: Mittels Membranisolierung und Western blotting wurde die Expression von EGFR, Her-2/neu, Her-3, Her-4 und ihrer Liganden EGF, TGF-a und HRG in 74 invasiv duktalen Mammakarzinomen analysiert (optical density, high re solution scanning), und mit klinischen Parametern wie Hormonrezeptorstatus, Tumorgrading oder Lymphknotenstatus korreliert. Ergebnisse: In $79,7 \%$ der Fälle wurden alle ErbB-Rezeptoren und in $82,4 \%$ alle $3 \mathrm{Li}$ ganden exprimiert. EGFR und Her-3 $(\mathrm{p}=0,005)$ sowie Her-3 und Her- 4 $(\mathrm{p}=0,05)$ waren signifikant ko-exprimiert, wobei die signifikanteste Korrelation für die simultane Expression von Her-2/neu und Her-3 ( $p=0,001)$ und Her-2/neu und Her-4 ( $\mathrm{p}=0,001)$ beobachtet wurde. Die Ko-Expression von EGFR und Her-3 war mit dem Vorhandensein aller 3 Liganden assoziiert, während HRG gleichzeitig mit Her-2/neu und Her-3 exprimiert wurde $(p=0,002)$. Patientinnen mit Lymphknotenmetastasen wiesen deutlich höhere Ko-Expressionsraten (EGFR-Her-3, p = 0,012; Her-2/ neu-Her-3, p<0,001; Her-2/neu-Her-4, p = 0,002) auf, während bei nodalnegativen Patientinnen lediglich Her-2/neu-Her-3 ( $\mathrm{p}=0,01)$ signifikant ko-exprimiert wurden. Hormonrezeptorstatus, Tumorgröße oder Grading verhielten sich unabhängig zur Expression der Rezeptoren oder Liganden. Schlussfolgerung: Die vorliegende Arbeit gibt erstmals Aufschluss über ein ko-reguliertes ErbB Rezeptor/Liganden-System in vivo. Dabei scheint das Her-2/neu Molekül der bevorzugte Partner für biologisch aktive Heterodimere zu sein, was durch die unterschiedlichen Expressionsmuster bei nodal-positiven und nodal-negativen Patientinnen unterstrichen wird.

\section{P89}

Persistierende Tumorzellen (PTZ) im Knochenmark von Brustkrebspatientinnen als Surrogatmarker für ein erhöhtes Rezidivrisiko in der onkologischen Nachsorge eine Langzeitanalyse

*Wolfgang Janni ${ }^{1}$, Brigitte Rack ${ }^{1}$, Christian Schindlbeck ${ }^{1}$ Stephan Braun ${ }^{2}$, Klaus Pantel' ${ }^{3}$, Maja Heinrigs ${ }^{1}$, Bernd Gerber ${ }^{1}$, Harald Sommer ${ }^{1}$, Udo Jeschke ${ }^{1}$, Klaus Friese ${ }^{1}$

${ }^{1}$ I. Frauenklinik der LMU München, München, Deutschland

2Universitätsklinikum für Frauenheilkunde,

Leopold-Franzens-Universität, Innsbruck, Austria

Institut für Tumorbiologie, UKE, Hamburg, Deutschland

Zielsetzung: Eine aktuelle Pool-Analyse hat die unabhängige prognostische Relevanz disseminierter Tumorzellen im Knochenmark (KM) zum Zeitpunkt der Mammakarzinomprimärdiagnose etabliert. Die vorliegende Studie untersuchte, ob die Untersuchung des KM im Rahmen der onkologischen Nachsorge eine Einschätzung des individuellen Rezidivrisikos nach Abschluss der Primärtherapie ermöglicht. Material und Methoden: Im Zeitraum 1/1994 bis 4/2003 wurde im Rahmen der onkologischen Nachsorge bei 228 Patientinnen und einem Median von 21,3Monaten (Standardabweichung [std] 29,1) nach Diagnose und Primärtherapie eines Mammakarzinoms im TNM-Stadium pT1-2pN0-3M0 eine KM-Punktion in Lokalanästhesie durchgeführt. Der Nachweis disseminierter Tumorzellen im KM wurde mit Hilfe des Panzytokeratinantikörpers A45-B/B3 und der APAAP-Färbemethode durchgeführt. Die mittlere Nachbeobachtungszeit betrug 49,8 Monate (std 32,1). Ergebnisse: Bei 29 Patientinnen $(12,7 \%)$ wurden PTZ bei der Nachpunktion detektiert. Die mittlere rezidivfreie Überlebenszeit betrug nach Kaplan-Meier Analyse 149,7 Monate $(139,6-159,8$ 95\% CI) für Patientinnen ohne, und 86,5 Monate (65,7-107,4 $95 \%$ CI, p = 0,0003, log-rank Test) für Patientinnen mit Nachweis von PTZ im KM. Das Gesamtüberleben unterschied sich signifikant zwischen den beiden Gruppen: 162,1 Monate (152,1-172,0) vs. 98,7 Monate (79,7-117,9 $\mathrm{p}=0,0008)$. In der multivariaten Cox-Regressions-Analyse für KMStatus, Tumorgröße, Nodalstatus und Grading bestätigte sich der KM-Status als unabhängiger Prognosefaktor für das weitere Überleben (RR 5,57, $\mathrm{p}=0,002)$. Die größte prognostische Aussagekraft einer KM-Nachpunktion konnte im Zeitraum zwischen 2 und 3,5 Jahren nach Primärdiagnose festgestellt werden (RR 7,68). Zusammenfassung: Der Nachweis von PTZ im KM könnte zukünftig als Surrogatmarker für die Notwendigkeit einer sekundär-adjuvanten Therapie dienen. Diese Information könnte besonders in Hinblick auf die derzeitige Diskussion zur endokrinen Therapie beim Mammakarzinom von Bedeutung sein. 
P 90

\section{Höhere Knochendichte bei Patientinnen mit primärem Mammacarcinom-Ultraschallmessung am Kalkaneus}

*Bernhard Krämer, Carmen Mack, Jens Huober, Diethelm Wallwiener, Erich-Franz Solomayer

Universitätsfrauenklinik, Tübingen, Deutschland

Ziel/Hintergrund: Patientinnen mit primärem Mammacarcinom haben ein erhöhtes Risiko für Osteoporose und Knochenmetastasen. Daher ist ein einfaches Knochendichtescreening für diese Gruppe erforderlich. Wir untersuchten die Knochendichte von 210 prä-/peri- und postmenopausalen Patientinnen (31-84J.) in Korrelation zu Tumorgröße, BMI, Grading, Nodalstatus, Ki-67, disseminierten Tumorzellen im Knochenmark und Rezeptorpositivität (Östrogen/Progesteron). Material und Methoden: Be 95 prä-/peri- und 115 postmenopausalen Patientinnen wurden mittels Kalkaneusultraschall SOS, BUA und stiffness index bestimmt. Präoperativ war eine Knochenmarkspunktion erfolgt. Ergebnisse: Der mediane T-Score war in der prä-/perimenopausalen Gruppe -0,37 (min: -2,37, max: 2,70 ), in der postmenopausalen -1,38 (min: -5,19, max: 1,56). Keine Korrelation in beiden Gruppen bestand zwischen Knochendichte und Tumorzelldissemination, Nodalstatus, Ki-67 und Hormonrezeptoren ( $>>0,05)$ Der mediane T-Wert für T1-Tumore war -0,675, für T2 -1,269 und fü T3/T4 -2,047 in der Gesamtgruppe $(p<0,05)$. Vergleichbare Ergebnisse fanden sich für die postmenopausale Gruppe bei alleiniger Bestimmung ( $p<0,05)$. Zusammenfassung: Die Knochendichte ist relativ hoch beim primären Mammacarcinom, höhere Dichte korreliert mit kleinerem Tumor. Keine Korrelation wurde für die anderen Parameter gefunden.

\section{P91}

\section{Effizienter Gentransfer in Mammakarzinomzellinien mittels AAV (Adeno-assoziiertes Virus) Genvektoren}

${ }^{*}$ Christian Kurzeder ${ }^{1}$, Bernd Koppold ${ }^{1}$, Hildegard Buening ${ }^{2}$ Georg Sauer ${ }^{1}$, Clemens Wendtner ${ }^{3}$, Michael Hallek ${ }^{3}$, Rolf Kreienberg ${ }^{1}$, Helmut Deissler ${ }^{1}$

${ }^{1}$ Universitätsfrauenklinik UIm, UIm, Deutschland

${ }^{2}$ Genzentrum der Ludwig-Maximilians Universität München,

München, Deutschland

${ }^{3}$ Medizinische Klinik I, Universität zu Köln, Köln, Deutschland

Zielsetzung: Innovative therapeutische Ansätze basierend auf einem Gentransfer immunstimulatorischer, tumorsuppressiver oder pro-apoptotischer Gene könnten herkömmliche Therapien beim Mammakarzinom sinnvoll ergänzen bzw. helfen regelmäßig beobachtete Chemotherapieresistenzen zu überwinden. Ziel des Projektes war es AAV-Genvektorüberstände der neuesten Generation hinsichtlich ihrer Eignung für den Gentransfer in Mammakarzinomzelllinien zu untersuchen, und die für den viralen Gentransfer relevanten zellulären Rezeptoren zu analysieren Materialien und Methoden: Rekombinante, EGFP (enhanced green fluorescent protein)-kodierende AAV-Genvektorüberstände wurden adenovirusfrei nach einem optimierten Protokoll generiert. Nach Transduktion verschiedener Mammakarzinomzelllinien wurde die Transgenexpression mittels Durchflusszytometrie quantifiziert und mit der Expression relevanter Rezeptoren für eine AAV Infektion korreliert. Ergebnisse: Mittels eines optimierten Verpackungsprotokolles für rAAV/EGFP konnte ein infektiöser Titer von $6 \times 109$ infektiöser Partikel pro ml erzielt werden. In 8 untersuchten Mammakarzinomzellinien war die erzielte Gentransfereffizienz hochvariabel und lag zwischen 4,5 und 93,3\%. Entgegen bisheriger Berichte ist die Expression der bekannten Rezeptoren HSPG, FGF-R, PDGF-R und alphaVbeta5 Integrine keine notwendige Voraussetzung für eine AAV Infektion dar und die Expression der Rezeptoren korellier nicht wesentlich mit der Infizierbarkeit. Zusammenfassung: AAV ist ein nicht-humanpathogenes Virus, AAV-Genvektoren besitzen deshalb ein herausragendes Sicherheitsprofil. Die erzielten Transduktionsraten in Mammakarzinomzellen sind viel versprechend und rechtfertigen eine Evaluation im Tiermodell. Unklar ist welches die relevanten Rezeptoren für einen AAV Gentransfer sind. Ein Ansatz für zuküftige klinische Anwendungen stellt das Rezeptortargeting durch gezielte Mutation des viralen Kapsides dar.

\section{P92}

Detektion von cytokeratin-positiven Zellen im Blut und ihr Einfluß auf das Überleben von Brustkrebspatientinnen

${ }^{*}$ Antje Lebrecht ${ }^{1}$, Eva Ulbrich ${ }^{2}$, Helge Taubert ${ }^{3}$, Udo Bilkenroth $^{3}$, Heinz Kölbl ${ }^{1}$

${ }^{1}$ Klinik und Poliklinik für Gynäkologie und Geburtshilfe,

Johannes Gutenberg-Universität, Mainz, Deutschland

${ }^{2}$ Klinik und Poliklinik für Gynäkologie, Martin-Luther-Universität, Halle Wittenberg, Deutschland

${ }^{3}$ Institut für Pathologie, Martin-Luther-Universität, Halle Wittenberg, Deutschland

Zielsetzung: Bisher konzentrierte sich die Suche nach Mikrometastasen auf das Knochenmark von Brustkrebspatientinnen. Wir untersuchten das Blut von Mammakarzinom-Patientinnen auf das Vorliegen von cytokeratin(ck)-positiven Tumorzellen und korrelierten diese Daten mit klinischen Parametern. Materialien und Methoden: Bei 83 Frauen mit Mammakarzinom erfolgte eine venöse Blutentnahme mit jeweils $16 \mathrm{ml}$ EDTA-Blut. Mittels Dichtegradientenzentrifugation und magnetischer Zellseparation (autoMACS, Milteny) wurden Tumorzellen isoliert und danach immunzytochemisch phänotypisiert. Ergebnisse: Zytokeratin-positive Zellen wurden im Blut bei 0 von 15 gesunden Probanden und bei 29 von 83 Brustkrebspatientinnen (35\%) gefunden. Das Auftreten von Zytokeratin-positiven Tumorzellen korrelierte signifikant mit dem Vorliegen von Lymphknotenmetastasen $(p=0,009)$ oder von Fernmetastasen $(p=0,003)$. Das mittlere Überleben bei Patientinnen mit cytokeratin-positiven Zellen im Blut betrug 50,9 Monate (95\% confidence interval 39,2-62,7) verglichen mit 67,8 Monaten (95\% confidence interval 42,5-93) bei Patientinnen ohne ck-positive Zellen im Blut. In der Gruppe der metastasierten Patientinnen $(\mathrm{n}=15)$ war die TTP bei Patientinnen mit cytokeratin-positiven Zellen im Blut statistisch signifikant schlechter $(\mathrm{p}=0,035)$. Zusammenfassung: Das Auftreten von ck-positiven Tumorzellen im Blut von Brustkrebspatientinnen scheint ein Marker für den Progress der Erkrankung zu sein.

P93

Konkordanz der HER-2/neu-Überexpression von primären Mammakarzinomen und ihren metachronen Fernmetastasen

* Diana Lüftner ${ }^{1}$, Heike Dilk ${ }^{1}$ Petra Henschke ${ }^{1}$, Rita Geppert ${ }^{1}$ Manfred Dietel ${ }^{2}$, Klaus-Dieter Wernecke ${ }^{3}$, Kurt Possinger ${ }^{1}$ ${ }^{1}$ Medizinische Klinik II, Charité Campus Mitte, Humboldt-Universität, Berlin, Deutschland

${ }^{2}$ Institut für Pathologie, Charité Campus Mitte,

Humboldt-Universität, Berlin, Deutschland

Institut für Medizinische Biometrie, Charité Campus Virchow, Humboldt-Universität, Berlin, Deutschland

Zielsetzung: Das Dogma der Klonalität zwischen Primärtumor und metachroner Fernmetastase wird zunehmend aufgeweicht. Die Diagnose der HER-2/neu-Positivität, meist am Primärtumor gestellt, ist die Voraussetzung für die Therapie mit Trastuzumab in der metastasierten Situation. Klonale Veränderungen im Erkrankungsverlauf könnten somit zur falschen Patientenselektion führen. Materialien und Methoden: Wir suchten in 2 Pathologiearchiven nach Paraffinblöcken von Mammakarzinomen und ihren jeweiligen metachronen Fernmetastasen. Insgesamt wurden 80 Paare aus dem Zeitraum von 1994-2003 auf die HER-2/neuExpression mit dem DAKO HercepTest gefärbt. Ergebnisse: Die Tumorcharakteristika waren wie folgt verteilt: $73 \%$ invasiv duktal, T1/2-Tumore 45/37\%, N1/2-Stadium 48/42\%. Die Biopsien erfolgten an folgenden Fernmetastasen: Viscera 9\%, Knochen 10\%, Weichteil 78\%, Rest andere. Insgesamt waren $47,7 \%$ der Primärtumore HER-2/neu-positiv (DAKO $+2 / 3$ ), während $59 \%$ der Fernmetastasen eine $+2 / 3$-Expression aufwiesen. Die Konkordanz zwischen der HER-2/neu-Expression der Primärtumore und ihrer Fernmetastasen war nur moderat gut mit einem Konkordanzindex kappa von 0,52. Der McNemar-Test für Veränderungen von HER2/neu-negativen Primärtumoren zu HER-2/neu-positiven Metastasen (17,9\% der Paare) im Vergleich zur umgekehrten Veränderung von HER2/neu-positiven Primärtumoren zu HER-2/neu-negativen Metastasen (6,4\% der Paare) erbrachte einen p-Wert von 0,063 zugunsten einer Häufung der Veränderung von negativen Primärtumoren zu positiven Meta- 
stasen und verpaßte damit knapp die statistische Signifikanz. Zusammenfassung: Klonale Veränderungen der HER-2/neu-Expression, gemessen mit dem immunhistochemischen HercepTest, sind häufiger, als dies allgemein angenommen wird. Der aktuelle, klinische Standard muß allerdings nicht revidiert werden. Frühere Untersuchungen zeigen, daß ein HER2/neu-Serumergebnis von $>50 \mathrm{ng} / \mathrm{ml}$ in der metastasierten Situation eng mit der HER-2/neu-Gewebeexpression korreliert und die Reliabilität der HER-2/neu-Diagnostik verbessert. Die Proben werden weiter mit der FISH-Diagnostik bearbeitet.

\section{P94}

Immuntherapie mit dendritischen Zellen; Phagozytoseaktivität dendritischer Zellen von Mammakarzinompatientinnen

${ }^{*}$ Constanze Matthes ${ }^{1}$, Dagmar Marx ${ }^{1}$, Dirk R. Lorenzen ${ }^{1}$,

Nicole Cillien ${ }^{1}$, J. Hinrich Peters ${ }^{2}$, Thomas Nesselhut ${ }^{1}$

${ }^{1}$ Institut für Tumortherapie, Duderstadt, Deutschland

${ }^{2}$ Universität Göttingen Abteilung für Immunlologie, Göttingen,

Deutschland

Eine Immuntherapie mit dendritischen Zellen beim Mammakarzinom kann eine spezifische gegen den Tumor gerichtete zelluläre Immunantwort mittels zytotoxischer T-Zellen induzieren. Vorrausetzung hierfür is unter anderem eine effiziente Aufnahme des Antigens durch dendritische Zellen sowie die Expression von MHC-Klasse-I/II- und von Adhäsionsmolekülen auf den Tumoren.

Bei Patientinnen mit metastasiertem Mammakarzinom wurden dendritische Zellen aus frisch isolierten Monozyten in vitro mittels Zytokine (IL-4, GM-CSF) ausdifferenziert und mit einem autologen TumorzellLysat gepulsed. Begleitend wurden Analysen zur Morphologie, zum Phänotyp(Durchlußzytometrie) und zur Funktionalität (Phagozytoseassay) durchgeführt. Bei $\mathrm{n}=11$ Patientinnen wurde das Tumormaterial phänoty pisch auf MHC-K1 I/II, ICAM-1 und LFA-3 untersucht.Die aus dem peripheren Blut gewonnenen Monozyten von den Patientinnen lassen sich sowohl morphologisch als auch phänotypisch zu unreifen dendritischen Zellen ausdifferenzieren. Darüber hinaus konnte bei $\mathrm{n}=3$ Patientinnen gezeigt werden, dass im Mittel $82 \%$ der Zellen die FITC-markierten Zymosanparitikel phagozytieren können. Phänotypisch konnte nach der Phagozytose keine Veränderung in der Expression des CD14, HLA-DR und CD1a festgestellt werden. Die durchflußzytometrischen Analysen der Tumoren zeigte bei keinen der analysierten Tumoren einen vollständigen Expressionsverlust der MHC-Klasse-I/II-, sowie der Adhäsionsmoleküle ICAM 1 und LFA-3. Im Mittel expremierten 62\% der Zellen MHC KL I 21\% MHC KL II, 64\% ICAM-1 und 36\% LFA-3.Monozyten von Mammakarzinom-Patientinnen lassen sich zu unreifen dendritischen Zellen ausdifferenzieren und stehen somit für eine Immuntherapie zur Verfügung. Es konnte gezeigt werden, dass dendritische Zellen von Mamma karzinompatientinnen in der Lage sind effezient zu phagozytieren und somit Bestandteile des Tumorzell-Lysates aufnehmen können. Darüber hinaus zeigte keiner der analysierten Tumoren einen vollständigen Expressionsverlust der für die zytotoxischen T-Zellen relevanten Moleküle.

\section{P95}

\section{Bone Trap- Befunde bei Brustkrebspatientinnen unter} Stammzell-gestützter Hochdosischemotherapie

Svjetlana Mohrmann*, Gerhard Schütt, Ursula Koldovsky, Hans Georg Bender, Ulrike Nitz

Frauenklinik der Heinrich-Heine Universität, Wsg - Düsseldorf, Deutschland

Zielsetzung: Der Abfall der Knochendichte unter adjuvanter Chemothe rapie steht im engen Zusammenhang mit zytotoxsischen Effekten auf Osteoblasten und Osteoklasten.An einer Gruppe von Pat. mit einem Hochrisikomammakarzinom, die im Rahmen einer Phase III Studie mit einer Hochdosischemotherapie behandelt wurden, sollte das Ausmaß der Knochenresorption sowie eine Verlaufskontrolle des Knochenstoffwechsels untersucht werden. Material und Methoden: Wir zeigen die Daten von 55 Pat; 40 Pat sind krankheitsfrei, 15 haben ein Rezidiv erlitten oder sind verstorben. Neben bildgebender Diagnostik mittels Osteodensitome trie, wurde zur Charakterisierung des Knochenabbaus bzw. der Osteo- klastenaktivität der Bone-Trap Test angewendet. Dieser Test misst die Tartrat-resistente sauere Phosphatase (TRAP) als Markerenzym der Knochen- resorbierenden Osteoklasten. Dieses Enzym betseht aus zwei Isoformen (5a und 5b), wobei nur die, im Serum detektierbare, aktive Isoform $5 \mathrm{~b}$ der TRAP für Osteoklasten spezifisch ist. Im Gegensatz zur alkalischen Phosphatase (AP), die als Enzym-Marker für Osteoblastenfunktion gilt, zeigt die TRAP 5 b keine Abhängigkeit von der Leber- und Nierenfunktion. Die TRAP wurde vor der Therapie, 4-6 Wochen nach der Randomisierung sowie 2-3 J nach Randomisierung im Serum mittels Bone-Trap Tests bestimmt. Ergebnisse: 28 Pat mit einer Überlebenszeit von 4-6 J nach der Randomisation zeigten folgenden Verlauf des BoneTrap Testes: der Abbauparameter TRAP zeigt unter adjuvanter Hochdosischemotherapie einen signifikanten Unterschied im Vergleich zu den Messwerten vor der Therapie, ebenso besteht ein signifikanter Unterschied zwischen den Ergebnissen während der Chemotherapie und nach 4-6 J. Die ausführlichen Daten werden präsentiert. Zusammenfassung: Die oben aufgeführten signifikanten Messwertunterschiede bei dem Bone-Trap-Test lassen auf eine Erhöhung der Knochenumbauvorgänge während der Hochdosischemotherapie schließen. Diese Veränderungen sind nach 4-6 J nicht mehr nachzuweisbar.

\section{P96}

\section{Differentielle Proteomanalyse beim Mammakarzinom mittels 2D-PAGE und ProteoTope ${ }^{\circledR}$}

${ }^{*}$ Hans Neubauer ${ }^{1}$, Susan Clare ${ }^{2}$, Michael A. Cahill' ${ }^{3}$ Markus Kolbe 4 , Matthias Berth ${ }^{4}$, Karl Sotlar ${ }^{5}$, Alfred Nordheim ${ }^{6}$, Diethelm Wallwiener ${ }^{1}$, Raffael Kurek ${ }^{1}$

${ }^{1}$ Universitäts-Frauenklinik, Tübingen, Deutschland

${ }^{2}$ Department of Surgery, Indiana University School of Medicine, Indianapolis, USA

${ }^{3}$ ProteoSys AG, Mainz, Deutschland

${ }^{4}$ Decodon $\mathrm{GmbH}$, Greifswald, Deutschland

${ }^{5}$ Institut für Pathologie, Tübingen, Deutschland

${ }^{6}$ Abteilung für Molekularbiologie, Institut für Zellbiologie, Tübingen, Deutschland

Brustkrebs ist eine komplexe und heterogene Erkrankung, die durch größtenteils noch ungeklärte Veränderungen im Proteom von Tumorzellen verursacht wird. Daher kann die Untersuchung spezifischer Tumorproteome zu einer Verbesserung des aktuellen therapeutischen Standards führen. Das Ziel unserer Projekte ist die Charakterisierung spezifischer Proteome undissezierter Tumore bzw. mikrodissezierter Tumorzellpopulationen, um neue, klinisch relevante Zielproteine zu identifizieren. Als Ausgangsmaterial dienen kryokonservierte Gewebeproben abgestimmter Tumorstufen, die in einer Oracle Datenbank (TumorBase) verwaltet werden. Extrahierte Proteine werden mittels verschiedener 2D-PAGEMethoden dargestellt und massenspektrometrisch bestimmt.

Projekt I: Bestimmung der Östrogenrezeptor (ÖR)-abhängigen Proteinexpression

Da die genaue Funktion der ÖR-regulierten Proteinexpression und deren Einfluss auf die Proliferation von Tumorzellen und damit die Tumorprogression relativ unbekannt ist, wurden Gewebeprobenlysate von $26 \mathrm{~Pa}$ tientinnen mit unterschiedlichen ÖR-Staten eingesetzt, um neue ÖR-abhängige Proteine zu ermitteln. Dazu wurden die Lysate mittels 2D-PAGE (insgesamt $130 \mathrm{Gele}$ ) aufgetrennt und silbergefärbt. Anschließend wurden die Gelbilder zu Mastergelen vereinigt und ausgewertet (Delta 2D, Decodon). Differentiell exprimierte Proteine wurden detektiert, aus den Gelen isoliert und mittels MALDI-TOF identifiziert.

Projekt II: Analyse differentieller Sub-Proteome durch die Kombination von LCM und ProteoTope.

Homogene Zellpopulationen abgestimmter Progressionsstufen wurden

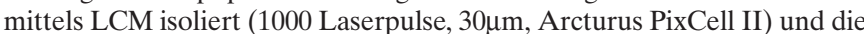
extrahierten Proteine mit Iodradiosisotopen (I-125, I-131) markiert. Dies ermöglicht ihre Fraktionierung mittels einer sehr sensitiven 2D-DIGE Plattform (ProteoTope, ProteoSys AG) sowie eine direkte differentielle Mehrfarbendarstellung. Mit ProteoTope können attomolare Proteinmengen kleiner aber homogener Zellpopulationen nachgewiesen werden. Differentiell dargestellte Proteine wurden mittels MALDI-TOF oder ESMS/MS präparativer Gesamttumorlysate bestimmt, die unmarkiert auf das gleiche Gel aufgetragen wurden. Ergebnisse beider Projekte werden präsentiert. 
P97

\section{N-Terminales Pro-Brain Natruretic Peptide (Bnp) als Prognosemarker während einer Langzeit-Immuntherapie mit Trastuzumab}

*Dagmar Rossner ${ }^{1}$, Karsten Knobloch ${ }^{2}$, Ralf Lichtinghagen ${ }^{3}$ Artur Lichtenberg ${ }^{2}$, Henning Kühnle ${ }^{1}$, Hans Joachim Lück ${ }^{1}$

${ }^{1}$ Brustzentrum, Medizinische Hochschule, Hannover, Deutschland ${ }^{2}$ Thorax-, Herz- und Gefäßchirurgie, Medizinische Hochschule,

Hannover, Deutschland

${ }^{3}$ Klinische Chemie, Medizinische Hochschule, Hannover, Deutschland

Trastuzumab (TR, Herceptin), ein selektiver HER-2(ErbB2)-Antikörper ist für die Therapie bei Patientinnen mit metastasiertem Brustkrebs zugelassen. Das N-terminale-pro-brain natriuretic peptide (Nt-pro-BNP) bildet den Schweregrad einer Herzinsuffizienz ab. Diese Arbeit untersuch die Konzentrationsverläufe von nt-pro-BNP während einer Langzeitimmuntherapie mit Trastuzumab bezüglich der Mortalität bei Brustkrebspatientinnen.

Bei 44 Patientinnen mit metastasierten, HER-2-positiven Mammacarcinomen wurden vor und 30 Minuten nach intravenöser Infusion von Trastuzumab Blutproben gewonnen. In Abhängigkeit von der nt-proBNP-Konzentration $<125 \mathrm{pg} / \mathrm{ml}$ [Gruppe $\mathrm{A}, \mathrm{n}=22$, Alter 53 (Bereich 22-78Jahre) vs. nt-pro-BNP $>125 \mathrm{pg} / \mathrm{ml}$ (Gruppe B, $\mathrm{n}=16$, Alter 64 (49-69Jahre), n.s.] bei HER-2-positiven Patientinnen sowie bei selektierten, HER-2 negativen Patientinnen, die nicht Herceptin erhielten $(n=6$ Alter 55 (29-81 Jahre, Gruppe C), wurden drei Gruppen definiert.

Die transthorakal echokardiographisch bestimmte ejection fraction $(\mathrm{EF} \%$ ) vor Therapiebeginn war $67 \pm 5 \%$ (A) vs. $67 \pm 7 \%$ (n.s.) (B), die niedrigste EF\% während der Trastuzumab-Therapie lag bei $61 \pm 8 \%$ (A, n.s.) vs. $56 \pm 11 \%(\mathrm{~B}, \mathrm{p}<0,05)$, die EF\% zum Zeitpunkt der nt-pro-BNP-Bestimmung betrug $62 \pm 7 \%$ (A, n.s.) vs. $59 \pm 6 \%$ (B, n.s.). Die Konzentrationen von nt-pro-BNP vor und nach Trastuzumabgabe waren $61 \pm 33$ vs. $67 \pm 30$ $\mathrm{pg} / \mathrm{ml}$ (n.s.) in Gruppe A vs. $369 \mathrm{pg} / \mathrm{ml}(159-1292 \mathrm{pg} / \mathrm{ml})$ vs. $336 \pm 294$ vs. $318 \pm 279 \mathrm{pg} / \mathrm{ml}$ in Gruppe B (n.s., p<0,05 A vs. B). Alle myokardialen Ischämiemarker (Kreatinkinase $\mathrm{CK}, \mathrm{CK}-\mathrm{MB}$ und Troponin $\mathrm{T}$ ) zeigten keine Veränderungen. Die mediane 3-Monats-Mortalität bezüglich der Brustkrebserkrankung betrug 4,5\% (1/22) in Gruppe A, 14,3\% in Gruppe B $(2 / 14)$ und $66,7 \%(4 / 6)$ in Gruppe $C(p<0,05)$.

Erhöhte nt-pro-BNP-Konzentrationen geben Hinweise auf eine erhöhte Mortalität bei Brustkrebspatientinnen. Während der Trastuzumabtherapie konnten keine akuten Veränderung der nt-pro-BNP-Konzentration beobachtet werden.

P98

HER-2/neu und Langzeitprognose beim Nodal-Negativen

Mammakarzinom: Immunhistochemie oder Fluoreszenz-In-Situ-Hybridisierung

*Marcus Schmidt ${ }^{1}$, Barbara Lewark ${ }^{1}$, Nikolai Kohlschmidt ${ }^{2}$, Eric Steiner ${ }^{1}$, Hans-Anton Lehr ${ }^{2}$ I, Wolfgang Weikel ${ }^{1}$, Heinz Kölbl ${ }^{1}$ ${ }^{1}$ Universitäts-Frauenklinik Mainz, Mainz, Deutschland

${ }^{2}$ Institut für Pathologie, Universitätsklinikum Mainz, Mainz, Deutschland

Zielsetzung: die Bedeutung von HER-2/neu beim nodalnegativen Mam makarzinom wird kontovers diskutiert. Studien, die mittels Immunhistochemie (IHC) die Überexpression nachgewiesen haben, stehen Arbeiten gegenüber, die mittels Fluoreszenz-in-situ-Hybridsierung (FISH) die Amplifikation untersucht haben. In der vorliegenden Arbeit wurden sowohl Überexpression als auch Amplifikation von HER-2/neu an einem Kollektiv von 101 adjuvant unbehandelten nodal-negativen Mammakarzinompatientinnen untersucht und mit traditionellen Prognosefaktoren verglichen. Methode: Die Überexpression von HER-2/neu wurde immunhistochmisch mit einem monoklonalen Antikörper (CB11), die Amplifikation mittels FISH mit einer Digoxigenin-markierten DNS Sonde nachgewiesen (HER2_AMP). Die IHC wurde sowohl qualitativ (HER2_EXP) als auch semiquantitativ (HER2_SCO) ausgewertet. Immunhistochemisch 2+ klassifizierte Tumore wurden zusätzlich auf die HER-2/neu Amplifikation untersucht (HER2_ALG). Außerdem wurde die Kombination aus Überexpression und Amplifikation untersucht (HER2_COM). Zusätzlich wurden Tumorgröße, histologischer Differenzierungsgrad und
Hormonrezeptorstatus für die Überlebenszeitanalysen berücksichtigt. Der Zusammenhang mit dem rezidivfreien und dem Gesamtüberleben wurde univariat und multivariat untersucht. Ergebnisse: HER-2_EXP war positiv in 30\%, HER-2_SCO 3+ in 20\%. Eine Amplifikation (HER2_AMP) wurde in $17 \%$ nachgewiesen. HER2_ALG war positiv in $22 \%$, HER2_COM in 11\%. Histologischer Differenzierungsgrad (Relatives Risiko (RR) 3,22, 95\% Konfidenzintervall (95\%CI) 1,73-5,99, p = 0,0002) und HER-2_ AMP (RR 2,47, 95\% CI 1,12-5,48, p = 0,026) waren für das rezidivfreie Überleben bei multivariater Analyse unabhängig signifikant. Für das Gesamtüberleben waren ebenso der histologische Differenzierungsgrad (RR 3,89, 95\%CI 1,77-8,55, $\mathrm{p}=0$,0007) und HER-2_AMP (RR 3,08, 95\% CI 1,24-7,66, p = 0,016) multivariat signifikant. Schlussfolgerung: die prognostische Bedeutung von HER-2/neu beim nodal-negativen Mammakarzinom hängt vom verwendeten Nachweisverfahren $a b$. Lediglich die Amplifikation von HER-2/neu hat eine unabhängige prognostische Aussagekraft für die Langzeitprognose.

P99

Eine Hochregulierung der Focal adhesion kinase (p125FAK)

in 162 nodal-negativen Mammakarzinomen ist mit einer HER-2/neu Überexpression und einer Akt-Aktivierung assoziiert, weist aber keine prognostische Relevanz auf

${ }^{*}$ Klaus Jürgen Schmitz ${ }^{1}$, Florian Grabellus ${ }^{1}$, Rainer Callies ${ }^{2}$, Friedrich Otterbach ${ }^{1}$, Jeremias Wohlschläger ${ }^{1}$, Kurt Werner Schmid ${ }^{1}$, Hideo Andreas Baba ${ }^{1}$

${ }^{1}$ Institut für Pathologie, Universität Essen-Duisburg, Essen, Deutschland

${ }^{2}$ Klinik für Geburtshilfe und Frauenheilkunde, Universität EssenDuisburg, Essen, Deutschland

Zielsetzung: Die Focal adhesion kinase (FAK) ist eine Tyrosin-Kinase, die Prozesse wie Wachstum, Differenzierung, Adhäsion, Motilität und Apoptose reguliert. Studien deuten darauf hin, dass eine FAK-Überexpression einen wichtigen Faktor in der Pathogenese und Progression von Tumorzellen inklusive Brustkrebs darstellt. Die Bedeutung der FAK-Kinase für die Prognose von Mammakarzinomen ist bislang unklar. Material/Methoden: Mittels Immunhistochemie haben wir die prognostische Relevanz der FAK-Expression an Paraffinmaterial von 162 nodal-negativen Mammakarzinomen(mean Follow up 7,48 Jahre) untersucht. Darüberhinaus wurde die FAK-Expression mit dem Aktivierungsstatus wichtiger Signalwege (HER2,Src,Akt,ERK1/2) verglichen und an einer kleinen Serie von frisch gefrorenem Mammakarzinomgewebe durch Western Blot die Expression von HER2, FAK sowie den aktivierten Formen phospho-FAK Tyr 861 und phospho-Src Tyr 215 ermittelt. Ergebnisse: Eine FAK-Überexpression zeigte keine prognostische Relevanz, jedoch fand sich eine signifikante Koexpression von FAK sowohl mit HER2 $(\mathrm{p}=0,009)$ als auch mit einer Aktivierung von $\operatorname{Src}(p=0,039)$ und Akt $(p<0,001)$. Die Western Blot Analyse ergab eine Korrelation der aktivierten Fom von FAK (pFAK Tyr 861) mit einer HER2-Überexpression $(\mathrm{p}=0,01)$. Unsere Daten bestätigen die Ergebnisse von Studien, die einen neuen Signalweg an Brustkrebszelllinien aufgedeckt haben, durch den eine HER2-Überexpression eine Aktivierung der FAK-Kinase an der Stelle Tyr 861 via Phosphorylierung von Src an der Stelle Tyr 215 erzeugt. Zusammenfassung: Zusammenfassend erwies sich die FAK-Expression nicht als prognostischer Marker in nodal-negativen Mammakarzinomen aber unsere Daten deuten darauf hin, dass eine HER2-Überexpression das biologisch maligne Verhalten von Mammakarzinomen mittels eines HER2-Src-FAK Signalweges beinflusst. Dies lässt die FAK Kinase zu einem interessanten Ansatzpunkt für eine potentielle therapeutische Intervention mittels sog. Designermolekülen werden. 
P100

Vergleich der Wirkung von Tamoxifen und 2-Methoxyestradiol auf die VEGF-Synthese in menschlichen Mammakarzinomzellen

Harald Seeger, Jens Huober, Diethelm Wallwiener, *Alfred O. Mueck Universitäts-Frauenklinik, Tübingen, Deutschland

Zielsetzung: Das Wachstum und die Metastasierung eines Tumors wird wesentlich von der Neoangiogenese bestimmt. Zu den wichtigsten proangiogenetischen Faktoren zählt der vaskuläre endotheliale Wachstums faktor (VEGF), der in verschiedene Zellarten synthetisiert werden kann. Es ist bekannt, dass Estradiol die Synthese von VEGF stimulieren kann und dass Tamoxifen eine Reduktion in vitro und in vivo bewirkt. In de vorliegenden Arbeit haben wir die Wirkung von Tamoxifen auf die VEGF-Synthese mit derjenigen von 2-Methoxyestradiol, einem endoge nen Estradiolmetaboliten mit antikanzerogenen Eigenschaften, verglichen. Materialien und Methoden: Die VEGF-Synthese wurde in MCF 7 Zellen, einem etablierten Zellmodel für weiblichen Brustkrebs, untersucht. Die Stimulierung von VEGF erfolgte durch 10 pM Estradiol, die Wirkung von 4-Hydroxytamoxifen, dem wirksamen Metabolit von Tamoxifen, und 2-Methoxyestradiol wurde allein und in Kombination mit Es tradiol in den Konzentration $10 \mathrm{nM}, 100 \mathrm{nM}$ und $1 \mu \mathrm{M}$ getestet. Die Inkubationszeit betrug 4 Tage, VEGF-Protein wurde im Zellüberstand mittels ELISA bestimmt. Ergebnisse: Estradiol bewirkte eine 60prozentige Erhöhung von VEGF. 4-Hydroxytamoxifen allein hatte keinen Effekt, wohingegen 2-Methoxyestradiol allein eine dosisabhängige Reduktion gegenüber der Kontrolle zeigte. In Kombination mit Estradiol waren beide Substanzen in der Lage die E2-induzierte Erhöhung aufzuheben, allerdings war der Effekt von 2ME um 20-30\% stärker als derjenige von Tamoxifen. Zusammenfassung: Der endogene Estradiolmetabolit, 2-Methoxyestradiol, kann die VEGF-Synthese von Mammakarzinomzellen un abhängig vom Estrogenrezeptor senken. Im Vergleich zu Tamoxifen ist die Wirkung von 2-Methoxyestradiol stärker. Dieser Mechanismus kann zur antikanzerogenen Wirkung dieses Metaboliten beitragen. Die Wirkung von Tamoxifen auf VEGF kann durch Kombination mit 2-Methoxyestradiol verstärkt bzw. bei reduzierter Sensitivität der Estrogenrezeptoren unterstützt werden.

\section{P101}

Die Expression verschiedener Marker der Angiogenese und Lymphangiogenese beim duktalen Carcinoma in situ (DCIS) der Mamma

*Pia Wülfing ${ }^{1}$, Christian Kersting ${ }^{2}$, Horst Bürger ${ }^{2}$, Björn Mattsson ${ }^{1}$, Christian Gustmann ${ }^{3}$, Bernd Hinrichs ${ }^{4}$, Joke Tio ${ }^{1}$, Werner Böcker ${ }^{2}$, Ludwig Kiesel ${ }^{1}$

${ }^{1}$ Universitätsfrauenklinik Münster, Münster, Deutschland

${ }^{2}$ Institut für Pathologie, Universitätsklinikum Münster, Münster,

Deutschland

${ }^{3}$ Institut für Pathologie, St. Vincenz-Krankenhaus, Limburg,

Deutschland

${ }^{4}$ Institut für Pathologie, Köln-Rodenkirchen, Deutschland

Zielsetzung: Wir untersuchten die Proteinexpression verschiedener Wachstumsfaktoren, die eine Rolle bei der Angiogenese und Lymphangiogenese spielen, sowie der zugehörigen Rezeptoren beim duktalen Carcinoma in situ (DCIS). Material und Methoden: Ein Tissue microarray (TMA) mit Gewebeproben von 200 DCIS (isoliertes DCIS: $\mathrm{n}=96$; koe xistentes DCIS: $\mathrm{n}=104$ ) wurde konstruiert und an Serienschnitten die Proteinexpression von bFGF, VEGF, Endothelin (ET)-1 und VEGF-C sowie der Rezeptoren bFGF-R1, Flt-1, ETAR, ETBR und Flt-4 immunhistochemisch bestimmt.Ergebnisse: Tumorzellen der meisten DCIS-Fälle zeigten eine Expression von VEGF-C, Flt-4 und ETAR (88\%, 95\% und $76 \%$ der Fälle), wohingegen eine bFGF und Flt-1 Expression der Tumorzellen nur sehr selten zu beobachten war (12\% und $16 \%$ der Fälle). VEGF-A, ET-1 und ETBR wurden bei einem Teil der DCIS-Fälle exprimiert (46\%, 48\% und 38\%). Beim isolierten DCIS war eine höhere (lymph)angiogenetische Aktivität mit vermehrter Expression von VEGFC, Flt-4 und ETAR häufiger als beim koexistenten DCIS zu finden. Dieser Effekt war in der Subgruppe der non-high-grade DCIS $(n=103)$ noch ausgeprägter als bei den high-grade DCIS. Zusammenfassung: Beinahe alle untersuchten DCIS-Läsionen exprimierten VEGF-C und Flt-4 als
Marker der Lymphangiogenese. Ferner zeigten die meisten DCIS-Fälle eine signifikante Expression verschiedener angiogener Faktoren und ihrer Rezeptoren. Dies deutet darauf hin, dass in situ Karzinome der Brust of fensichtlich in der Lage sind, Angiogenese und Lymphangiogenese zu induzieren. Die häufigere Expression angiogener Faktoren in der Gruppe der isolierten DCIS-Fälle vom non-high-grade Typ lassen vermuten, dass die Angiogenese ein relativ frühes Ereignis in der Karzinogenese des Mammakarzinoms darstellt und eher mit gut differenzierten als mit höhergradigen DCIS assoziiert ist.

\section{Poster: Varia \\ P102 \\ Ultraschallassistierte Operationstechniken an der Mamma zur \\ Verbesserung der Anatomiegerechten Präperation bei \\ Neoplasien (Schnittrand), bei Bet sowie bei histologisch \\ gesicherten Benignen Läsionen der Mamma - \\ unser interdisziplinäres Therapiekonzept seit 3 Jahren}

\section{Mario Marx}

Klinik für plastische und rekonstruktive Chirurgie,

Städtisches Klinikum GmbH Görlitz, Görlitz, Deutschland

Wir realisieren in unserer Klinik das gesamte Spektrum der Mammachirurgie, integriert in ein seit Mai 2001 etabliertes und an den Eusomaguidelines orientiert arbeitendes Mammazentrum. Wir behandeln in 2004 bisher 74 Primärerkrankungen. In 2003 realisierten wir 450 Mammarekonstruktionen. OP-Techniken: diep. lado.dufourmentel. onkolog.Redukktionsplastiken.usw.

Dr. Handstein arbeitet in der Bundesgeschäftsstelle- Qualitätssicherung Fachgruppe Mammachirurgie, als auch in der sächsischen Landesärztkammer -Dresden Fachgruppe Mammakarzinom aktiv.

Herr Handstein verfügt über die volle Weiterbildungsermächtigung unseres Fachgebietes.

Dr. Marx ist Coautor der s-3-leitliniender dgs - operative Behandlung und Leiter unseres Mammazentrums seit 3 Jahren.

\section{P103}

\section{Aderhautmetastase als klinische Primärmanifestation eines Mammakarzinoms}

*Heinz S. Scholz, Edgar Petru, Christoph Benedicic, Raimund Winter Geburtshilflich-gynäkologische Universitätsklinik Graz, Graz, Österreich

Einleitung: Aderhautmetastasen sind eine äußerst seltene Form der Fernmetastasierung eines Mammakarzinoms. Wir berichten über eine Patientin mit einem Mammakarzinom, das sich klinisch primär opthalmologisch manifestiert hatte. Fallbeschreibung: Eine 47-jährige Patientin war primär wegen Sehstörungen opthalmologisch abgeklärt worden. Dabei war ein Aderhauttumor links diagnostiziert, und der Verdacht auf ein metastatisches Geschehen ausgesprochen worden. Im Rahmen der Anamneseerhebung gab die Frau schließlich an, seit einigen Jahren einen Knoten in der rechten Brust bemerkt zu haben. Bei der Palpation fand sich im oberen äußeren Quadranten der rechten Brust ein gut pflaumengroßer, derber, schlecht verschieblicher Tumor mit Hauteinziehung. Im Rahmen der stanzbioptischen Sicherung fand sich ein invasiv-ductales Mammakarzinom, G3, mit hochgradiger Östrogenrezeptorpositivität (Score 8) und geringgradiger Progesteronrezeptorpositivität (Score 2). Eine Her-2-Neu Überexpression konnte nicht nachgewiesen werden. Im Rahmen der weiteren Staginguntersuchungen fanden sich pathologisch vergrößerte und sonographisch suspekte axilläre Lymphknoten bis $2,8 \mathrm{~cm}$, multiple Rundherde intrapulmonal und subpleural sowie multiple, pathologisch vergröBerte Lymphknoten in sämtlichen mediastinalen Lymphknotenstationen. In der Leber waren multiple parenchymersetzende Läsionen zu diagnostizieren. In der Skelettszintigraphie kamen keine Mehrspeicherungen im Sinne von Skelettmetastasen zur Darstellung. Therapeutisch wurde neben einer palliativen Radiotherapie des linken Auges ein konkomittierende Chemotherapie mit EC (Epirubicin $90 \mathrm{mg} / \mathrm{qm}$, Cyclophosphamid 600 mg/qm) alle 3 Wochen eingeleitet. Schlussfolgerung: Auch wenn das 
Mammakarzinom dieser Patientin zweifellos zu einem früheren Zeitpunkt erkannt hätte werden können, führte doch erst die Aderhautmetastase zur Diagnose und zu einer (palliativen) Therapie. Wenn im Rahmen der Manifestation einer Aderhautmetastase kein Primärtumor bekannt ist, sollte jedenfalls auch nach einem Mammakarzinom gesucht werden.

\section{P104}

\section{Epidemiologie des Brustkrebses beim Mann}

\section{R. Stabenow}

Gemeinsames Krebsregister der Länder Berlin, Brandenburg, Mecklenburg-Vorpommern, Sachsen-Anhalt und der Freistaaten Sachsen und Thüringen (GKR), Deutschland

Material und Methoden: Bevölkerungsbezogene Analysen von Mammakarzinomen beim Mann in Deutschland sind sehr selten. Das GKR ist mi einer Basispopulation von 17 Millionen das größte epidemiologische Krebsregister in Deutschland und verfügt über Daten beginnend mit dem Diagnosejahr 1961. Wir analysierten die Daten der Diagnosejahre 1999-2001. Es wurden Inzidenzraten, Stadienverteilungen, Sublokalisationen und histologische Tumortypen ausgewertet. Ergebnisse: Die Zah der jährlich an Brustkrebs erkrankenden Männer in Deutschland wird auf etwa 350 bis 400 geschätzt. Im Einzugsgebiet des GKR wurden im Zeitraum 1999-2001 221 invasive Mammakarzinome (ICD-10 C50) gemeldet. Das durchschnittliche Erkrankungsalter (Median) beträgt 66 Jahre. Mit einem Anteil von nur 0,2\% an allen Krebsneuerkrankungen bei Männern gehört diese Tumorart zu den äußerst seltenen Krebserkrankungen Die rohe Inzidenzrate beträgt $0,9 / 100000$ und die altersstandardisierten Raten $0,8 / 100000$ (ESR) bzw. 0,5/100 000 (WSR). Die altersstandardisierte Rate stieg seit Anfang der 60er Jahre (ESR 0,6/100 000) kontinuierlich an. 5\% aller Tumoren werden im in situ-Stadium diagnostiziert. Der Anteil der Tumoren $<10 \mathrm{~mm}$ (T0 bis $\mathrm{T} 1 \mathrm{~b}$ ) beträgt $15 \%$. Insgesamt gibt es keinen signifikanten Unterschied zur Stadienverteilung bei Frauen. Deutliche Unterschiede zu Mammakarzinomen bei Frauen dagegen gibt es erwartungsgenäß in der Lokalisation. Beim Mann entfallen 93\% aller Karzinome auf Brustwarze und Warzenhof. Die histologischen Tumortypen entsprichen auch in etwa der Klassifikation bei Frauen mit einem Anteil des duktalen Karzinoms von 83\%.

\section{P105}

\section{Weichteil-Tissue-Engineering: Der potentielle Einsatz von Präadipozyten-Fibrin-Konstrukten in der Mamma-Chirurgie}

*Nestor Torio-Padron, *Arash Momeni, Jörg Borges,

Niklas Baerlecken, G. Björn Stark

Abteilung für Plastische und Handchirurgie, Uniklinik Freiburg,

Freiburg, Deutschland

Ziel: Sowohl die Verwendung von alloplastischen Prothesen als auch Lappenplastiken sind bei der Brustrekonstruktion nach Mastektomie be Mammakarzinom nicht unproblematisch. Tissue Engineering könnte hier eine Alternative darstellen. Da das Volumen der weiblichen Brust durch schnittlich zu 70\% aus Fett besteht, haben wir untersucht, inwieweit kultivierte Präadipozyten als Weichteilersatz in Frage kommen. Methoden: Humane Präadipozyten wurden in einer Fibrinmatrix suspendiert und anschließend die Zellsuspension subkutan in die Flanken von Nacktmäusen injiziert. Nach 4 Wochen $(n=8), 3(n=8)$ und 6 Monaten $(n=8)$ wurden die Konstrukte explantiert und hinsichtlich der Formation reifen Fettgewebes sowie der Größenentwicklung der Konstrukte untersucht. Ergebnisse: $\mathrm{Zu}$ jedem Explantationszeitpunkt konnte neuformiertes reifes Fettgewebe nachgewiesen werden. Hinsichtlich des Wachstumsverhaltens konnte nach anfänglicher geringfügiger Größenabnahme der Konstrukte nach 4 Wochen, eine deutliche Größenzunahme verzeichnet werden. Diskussion: Die Beobachtung der Größenzunahme der PräadipozytenFibrin-Konstrukte zeigt die Möglichkeiten des Tissue Engineering, Konstrukte mit Proliferationstendenz zu generieren, welche als Weichteilersatz Verwendung finden könnten. Langfristig ist die klinische Anwendung dieser Methode sowohl in der rekonstruktiven, aber auch in der ästhetischen Mamma-Chirurgie denkbar.

\section{P106}

Unilaterale Gynäkomastie bei kontralateralem Poland-Syndrom eines 16jährigen Patienten - Case Report

*Anja Weidner ${ }^{1}$, Volker Duda ${ }^{1}$, Corinna Heitmann ${ }^{1}$, Eva Sax ${ }^{2}$, Uwe Wagner ${ }^{1}$, Karin Bock ${ }^{1}$

${ }^{1}$ Klinik für Gynäkologie, Gyn. Endokrinologie und Onkologie, Philipps-Universität Marburg, Marburg, Deutschland ${ }^{2}$ Klinik für Diagnostische Radiologie der Philipps-Universität Marburg, Marburg, Deutschland

Einleitung: Eine transiente ein- oder beidseitige Gynäkomastie wird in Phasen der hormonellen Umstellung wie der Pubertät häufig angetroffen. Das 1841 von Poland erstmalig beschriebene gleichnamige Syndrom ist gekennzeichnet durch unilaterale muskulo-skeletale Fehlbildungen des Thorax und der oberen Extremitäten, eine Hypo- bis Aplasie von Brustdrüse und Mamille sowie fakultativ weitere Begleitfehlbildungen. Ursächlich wird eine frühembryonale Durchblutungsstörung der Arteria subclavia und der vertebralen Arterien diskutiert, eine zusätzliche genetische Prädisposition wird vermutet. Wir berichten über den besonderen Fall eines 16-jährigen Adoleszenten mit einseitiger Gynäkomastie bei kontralateralem PolandSyndrom. Methode: Anlass zur Vorstellung des 16jährigen Adoleszenten in der Senologischen Sprechstunde war eine auffällige «Anisomastie». Die weitere Diagnostik bestand in der sorgfältigen klinischen Untersuchung, der standardisierten Mammasonographie $(7,5-13 \mathrm{MHz})$ sowie einer einseitigen Röntgenmammographie in einer Standardebene (Oblique). Ergebnisse: Die klinische Untersuchung zeigte einseitig eine Brustentwicklung äquivalent dem Thelarche-Stadium B3 nach Tanner. Auf der Gegenseite imponierte eine Aplasie des Drüsengewebes mit tiefsitzender, hypoplastischer Mamille. Sonographisch bestätigte sich die Verdachtsdiagnose der einseitigen Gynäkomastie und des kontralateralen Poland-Syndroms mit Aplasie der Pektoralis-Muskulatur und des Drüsengewebes. Mammographisch zeigte sich ebenfalls das typische Bild einer nodulären Gynäkomastie. Schlussfolgerung: Die überwiegend transiente, peripubertäre Gynäkomastie bedarf in der Regel keiner invasiven Diagnostik oder therapeutischen Intervention. Mit der standardisierten Mammasonographie (7,5-13 $\mathrm{MHz}$ ) läßt sich das Drüsengewebe gut gegen pathologische Herdbefunde abgrenzen. Auf die zusätzliche Mammographie kann bei eindeutigen Befunden überwiegend verzichtet werden. In Einzelfällen kann eine psychologische Betreuung der betroffenen jugendlichen Männer erwogen werden, wenn der Befund wie im vorliegenden Fall durch das kontralateral bestehende Poland-Syndrom besonders eindrucksvoll hervortritt.

\section{P107}

\section{Usability of Breast Cancer Web Sites}

\section{*Christian Weissenberger, D. Müller, S. Jonassen, M. Neumann,} J. Beranek-Chiu, S. Bartelt

Klinik für Strahlenheilkunde, Universitätsklinik Freiburg, Freiburg, Deutschland

Purpose: To evaluate the usability of health-related information for patients with breast carcinoma available on the Internet, measured as average search engine rank (ASER), average hit count (AHC), average number of links (ANL) and relevant information density (RID). Methods: Using a special software tool all the hits from 19 search engines were collected, providing a result of 10616 hits for the keywords 'breast cancer', 'Brustkrebs' or 'Mammakarzinom'. These hits were checked for dublettes and sorted in a database (date: 2001-03-01, 19:36). Then all URL addresses were visited and analysed with a specially developed questionnaire. Results: RID: Of 3,812 addresses in German language only 65 (1,7\%) and of 6,804 English language search results $131(1,9 \%)$ contain detailed information on Breast Cancer treatment. Information on special parts of the disease (e.g. mammography screening) is given in 8,6\% (German sites) and in 13,7\% (English und American sites). Owner of the relevant websites are institutions or organizations in $45 \%$, industry in $41 \%$ and private in $14 \%$. Additional web usability parameters: AHC 1,58, ANL 38,8, dead links 5,3\%, ASER 71,7, mean size of sites is $77,8 \mathrm{kB}$ (download time with analog modem more than 21,6 seconds). Conclusions: Just a few breast cancer web sites contain exact information about treatment options. These sites are hard to find in this mass of available search results. Web usability of informations for breast cancer patients is not sufficient (dead links, uncomfortable download time). 
P108

Rezidivierendes Angiosarkom nach brusterhaltender Therapie eines Mammakarzinoms

*Verena Friederike Zarghooni, Cordula Moers, Mathias Warm,

Peter Mallmann

Klinik für Gynäkologie und Geburtshilfe der Universität zu Köln, Köln, Deutschland

Angiosarkome stellen eine sehr seltene Tumorentität dar. Sie machen zirka 0,04 bis $1 \%$ aller bösartigen Erkrankungen der Mamma aus. Für die Entstehung wird vor allem eine radiogene Induktion durch vorrausgehende strahlentherapeutische Behandlung verantwortlich gemacht. Fallbe richt: 78-jährigen Patientin, Z.n. invasiv duktalem Mammakarzinom link (9/1996), pT2 pN0 M0, G2, Hormonrezeptor positiv. Es erfolgte eine brus terhaltende Therapie und Axilladissektion, Radiatio und adjuvante The rapie mit Tamoxifen über 5 Jahre. 7 Jahre nach Erstdiagnose entwickelte sich eine livide Hautverfärbung, die den gesamten unteren inneren Qua- dranten der linken Mamma einnahm. Eine PE erbrachte den Nachweis eines Angiosarkoms (6/03). Es erfolgte die Ablatio simplex der linken Mamma. Die histologische Aufarbeitung des Mammaabladates zeigte ein ausgedehntes Angiosarkom mit einer Ausdehnung von zirka $10 \times 6 \mathrm{~cm}$ überwiegend low-grade-Differenzierung. Die Resektion war im Gesunden erfolgt. Die Staginguntersuchungen waren unauffällig. 1/04 stellte die Patientin sich erneut mit einer stecknadelkopfgroßen lividen Veränderung der Thoraxwand links vor. Eine Punch-Biopsie sicherte die Diagnose eines Rezidivs des low-grade-Angiosarkoms. Eine großzügige Exstirpation mit Verschiebelappenplastik erbrachte erneut eine R0-Resektion. 4/04 unterzog sich die Patientin auswärts einer ambulanten Operation in Lokalanästhesie bei erneutem Angiosarkom-Rezidiv mit subtotaler Resektion. Die Patientin lehnt derzeit jede weitere Therapie ab. Zusammenfassung: Angiosarkome der Brust treten meist als Zweitmalignome nach ionisierender Bestrahlung auf. Die Therapie der Wahl stellt die lokale vollständige Resektion dar. Die Prognose des Angiosarkoms ist schlecht, die 5-JÜR wird mit 25-30\% angegeben. 